\title{
Synthesis of L-Pyranosides by Hydroboration of
}

\section{Hex-5-enopyranosides Revisited}

\author{
Grzegorz Łopatkiewicz and Jacek Mlynarski* \\ Faculty of Chemistry, Jagiellonian University, Ingardena 3, 30-060 Krakow \\ jacek.mlynarski@gmail.com,www.jacekmlynarski.pl
}

\section{Contents}

NMR spectra of compounds 2a-r, 3a-c, 3e-j, 3l-p, 4a-r, 5-26

NMR spectra of crude mixtures of compounds $\mathbf{4 a - c}, \mathbf{4 e}, \mathbf{4 f}, \mathbf{4 j}, \mathbf{4 n}$ 


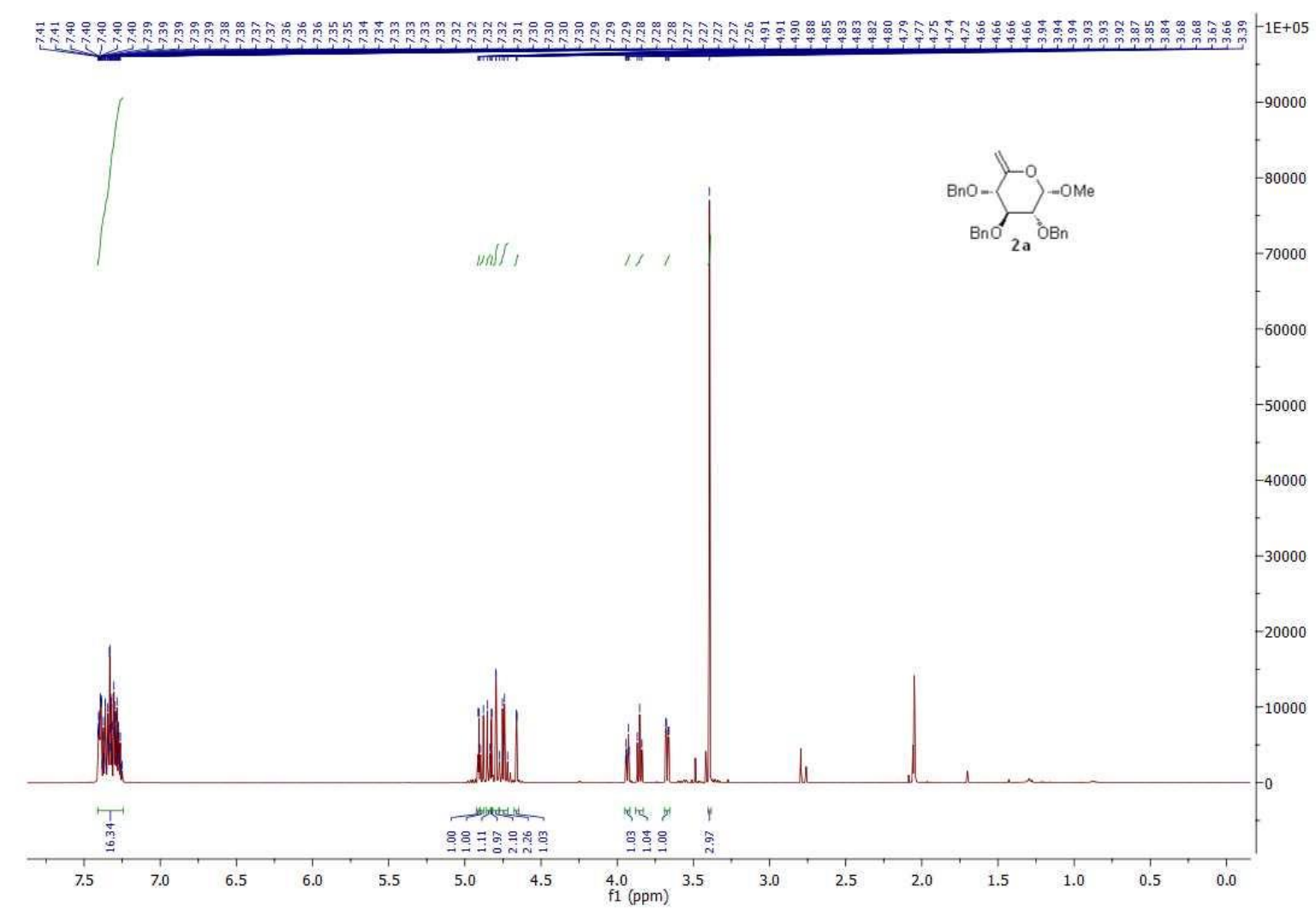

${ }^{1}$ H-NMR Spectrum of Compound 2a (Acetone-d6)

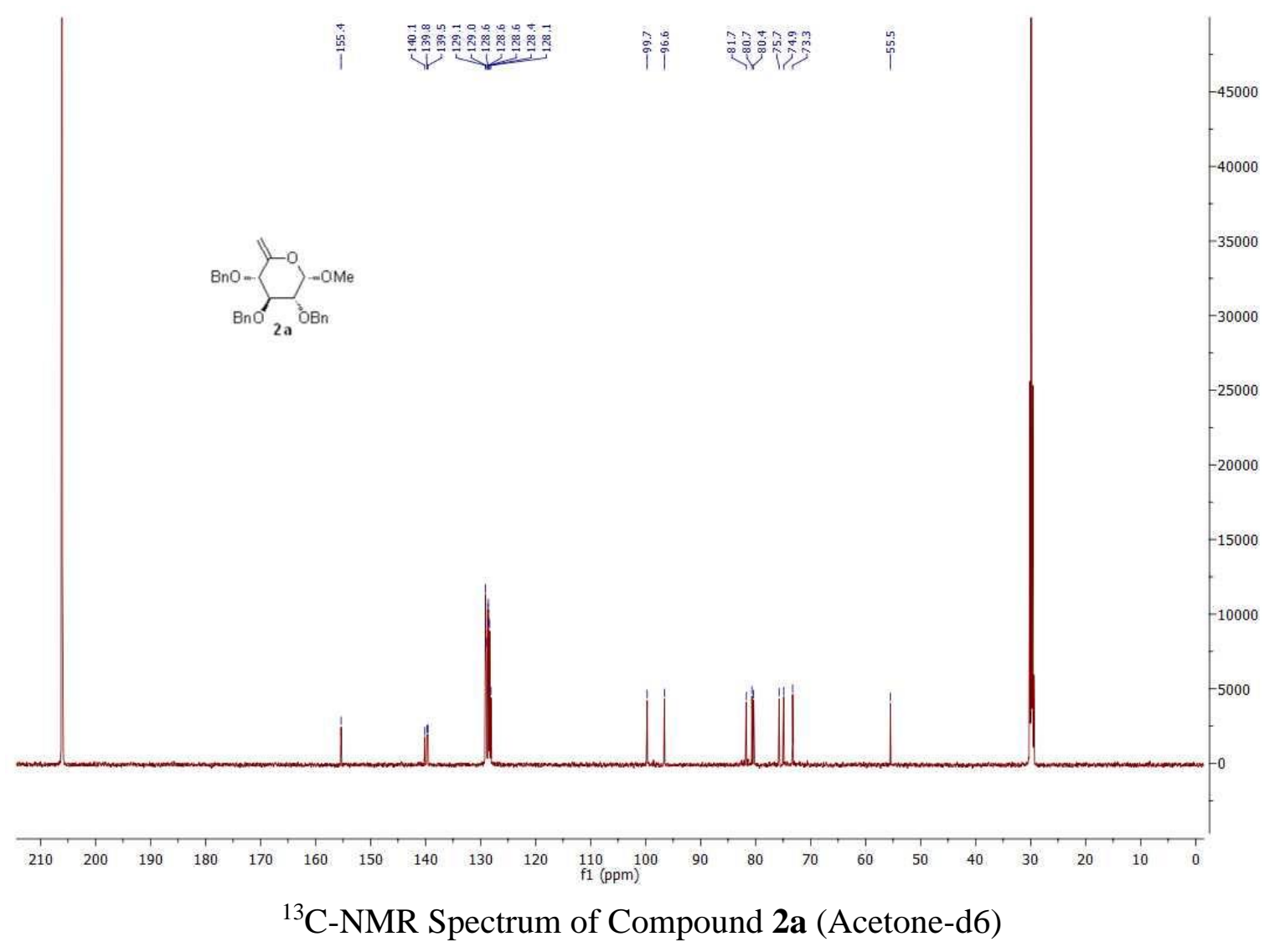




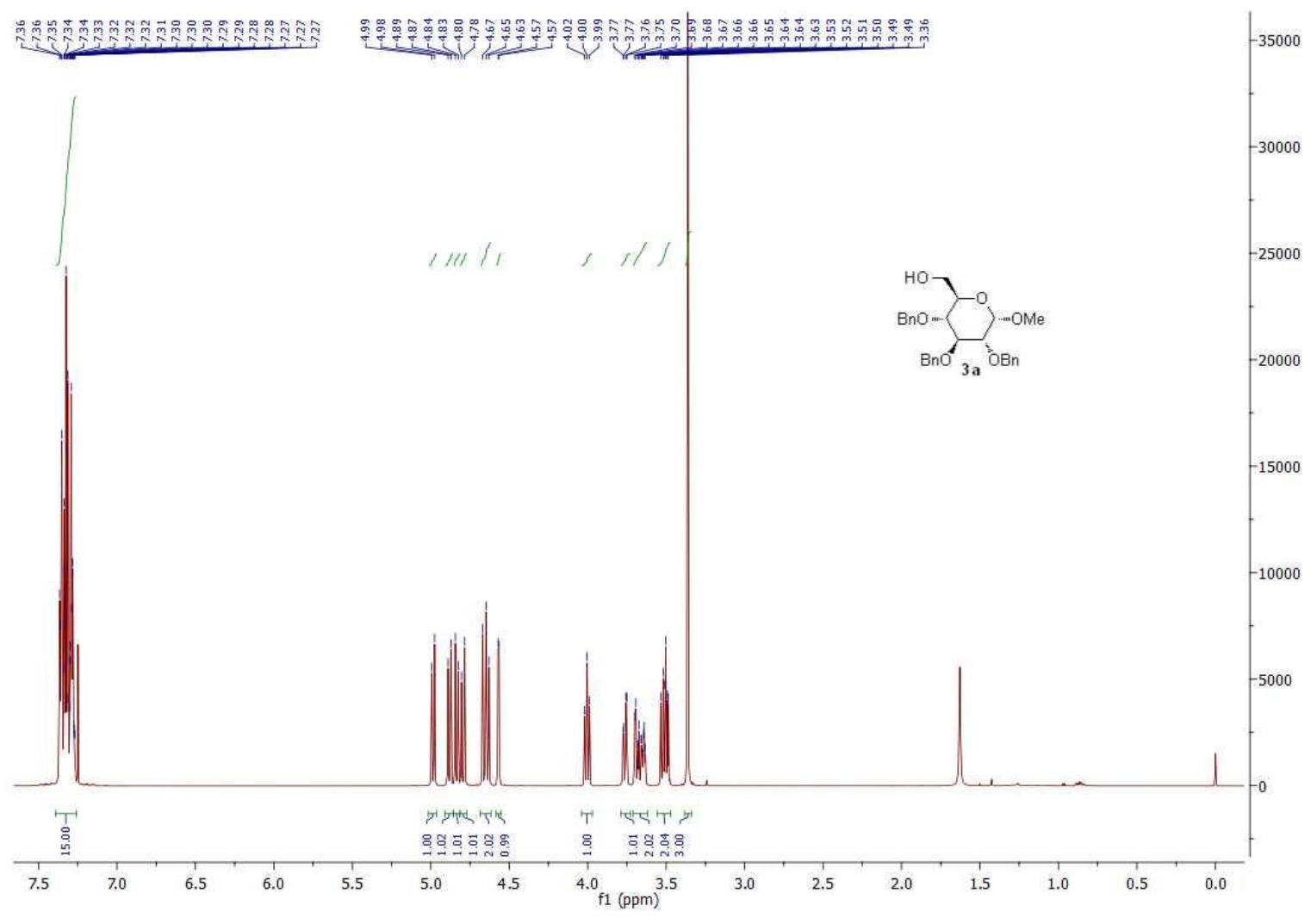

${ }^{1} \mathrm{H}-\mathrm{NMR}$ Spectrum of Compound $\mathbf{3 a}\left(\mathrm{CDCl}_{3}\right)$

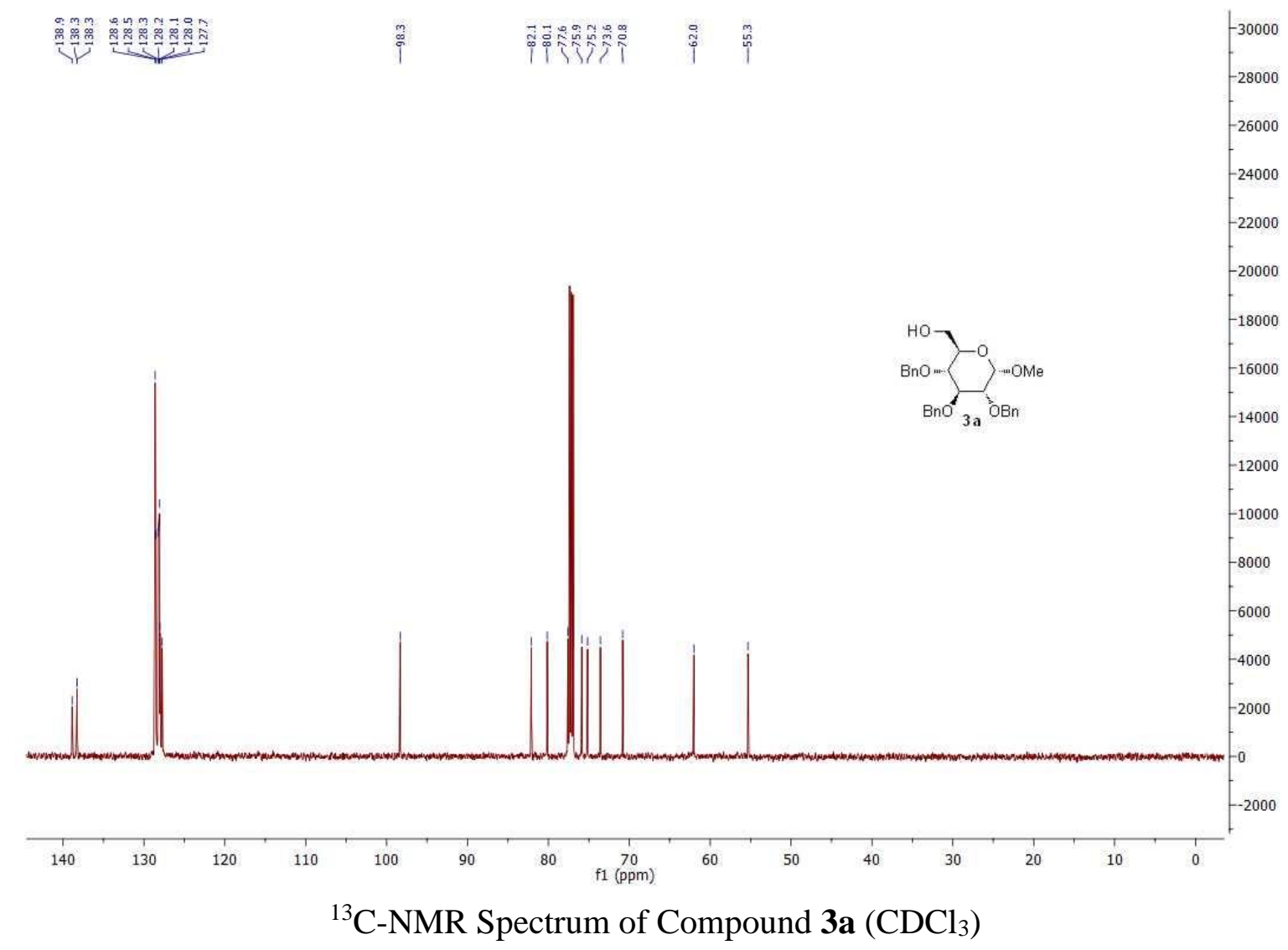




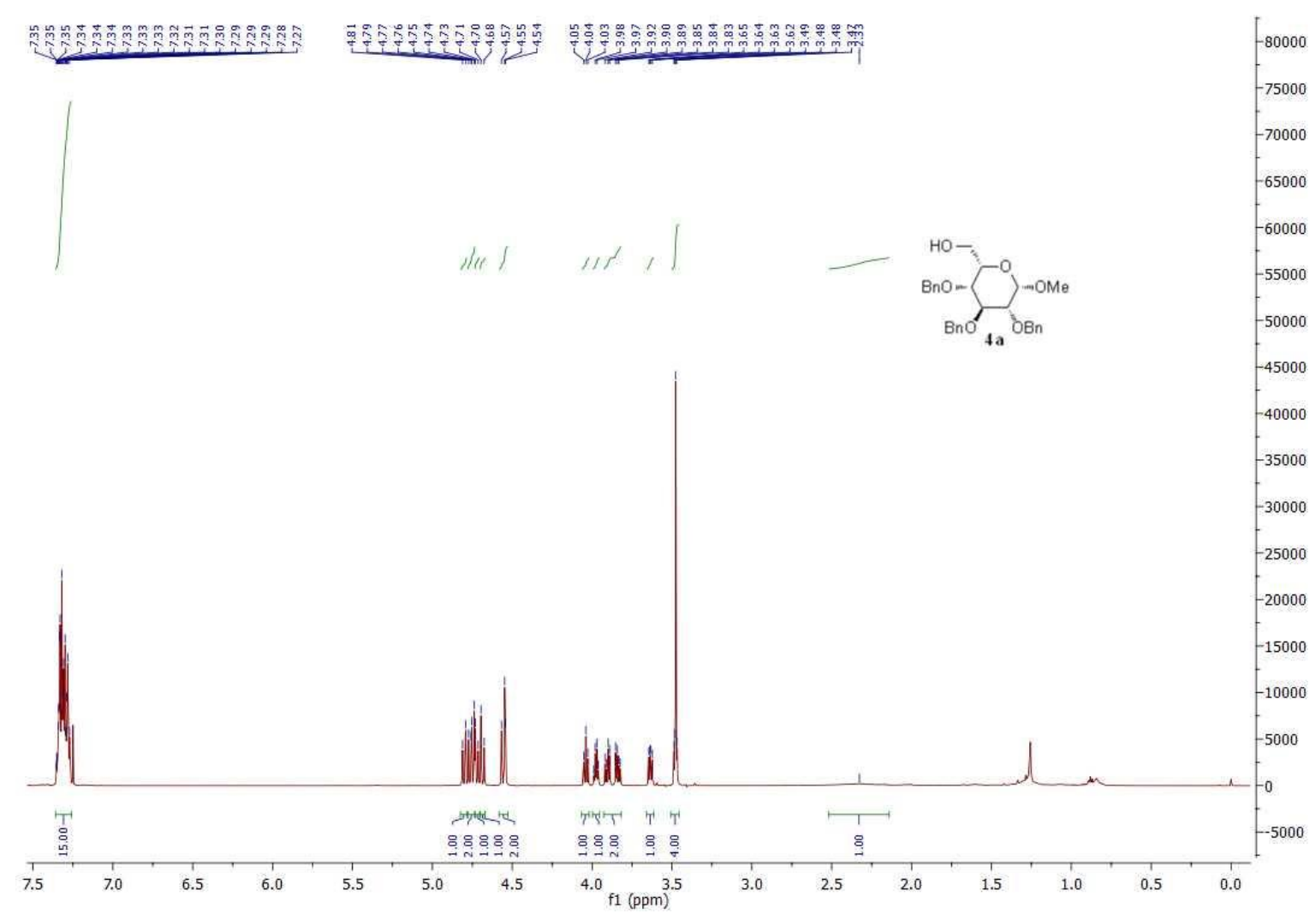

${ }^{1} \mathrm{H}-\mathrm{NMR}$ Spectrum of Compound $\mathbf{4 a}\left(\mathrm{CDCl}_{3}\right)$

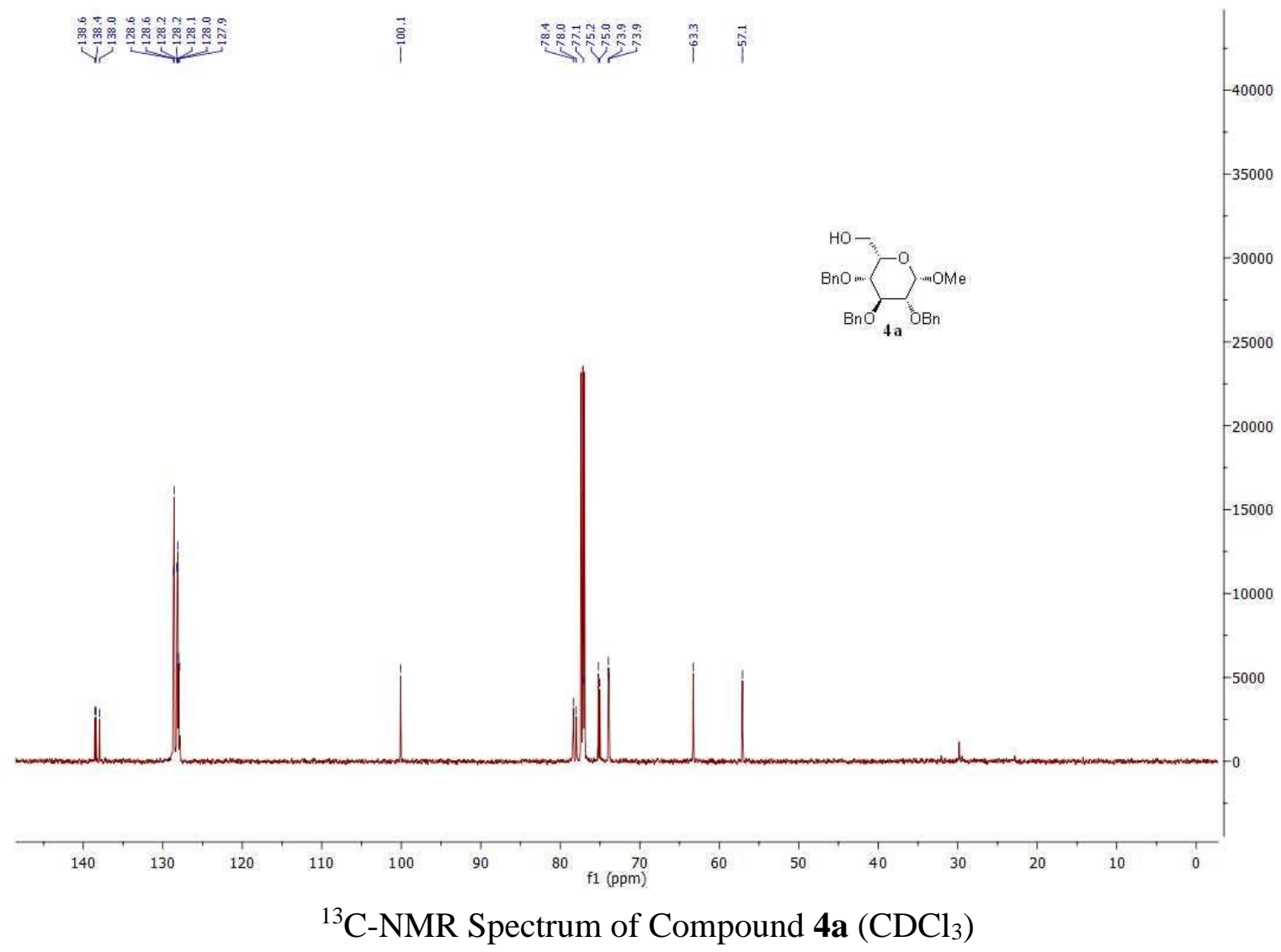




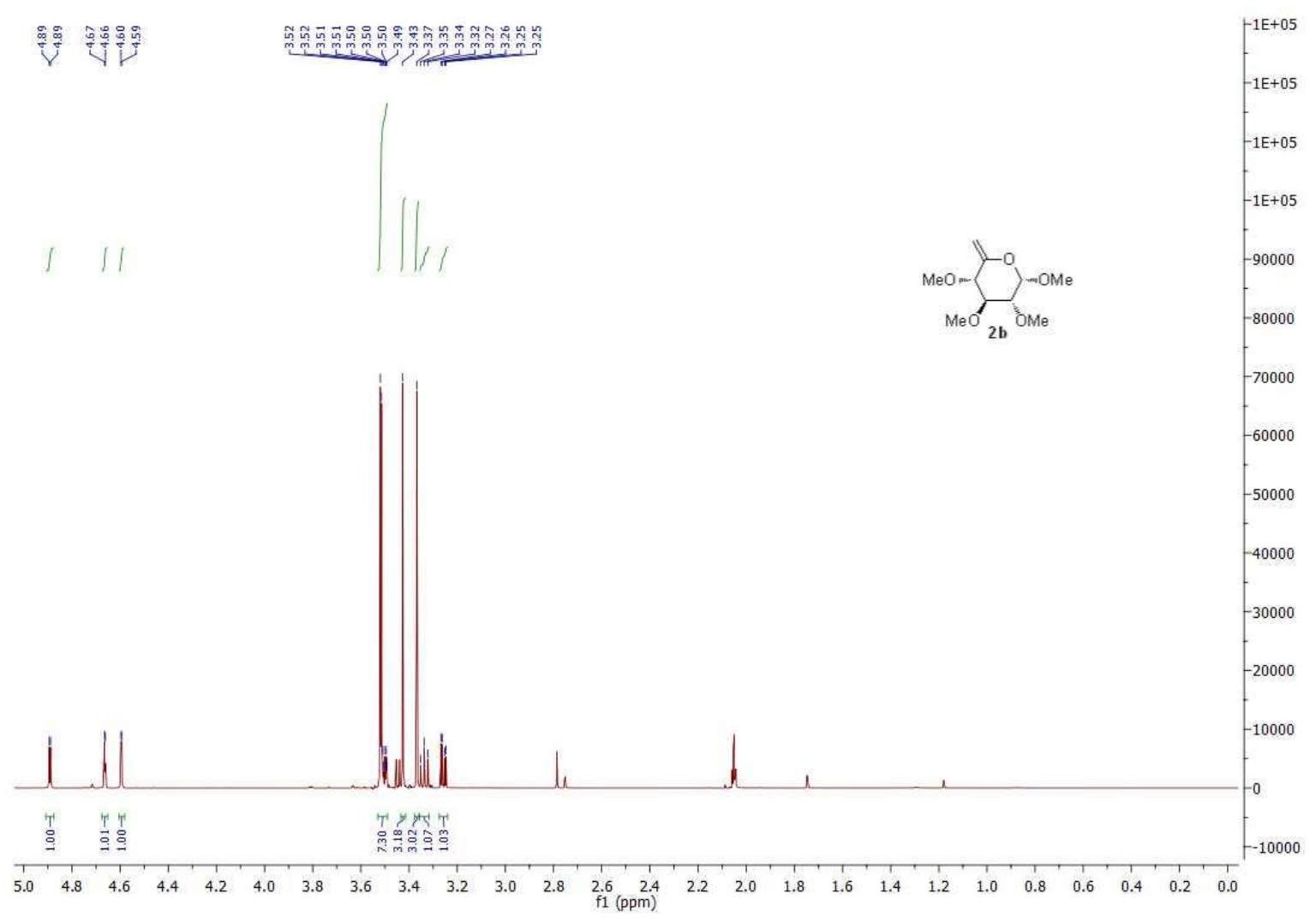

${ }^{1}$ H-NMR Spectrum of Compound $\mathbf{2 b}$ (Acetone-d6)

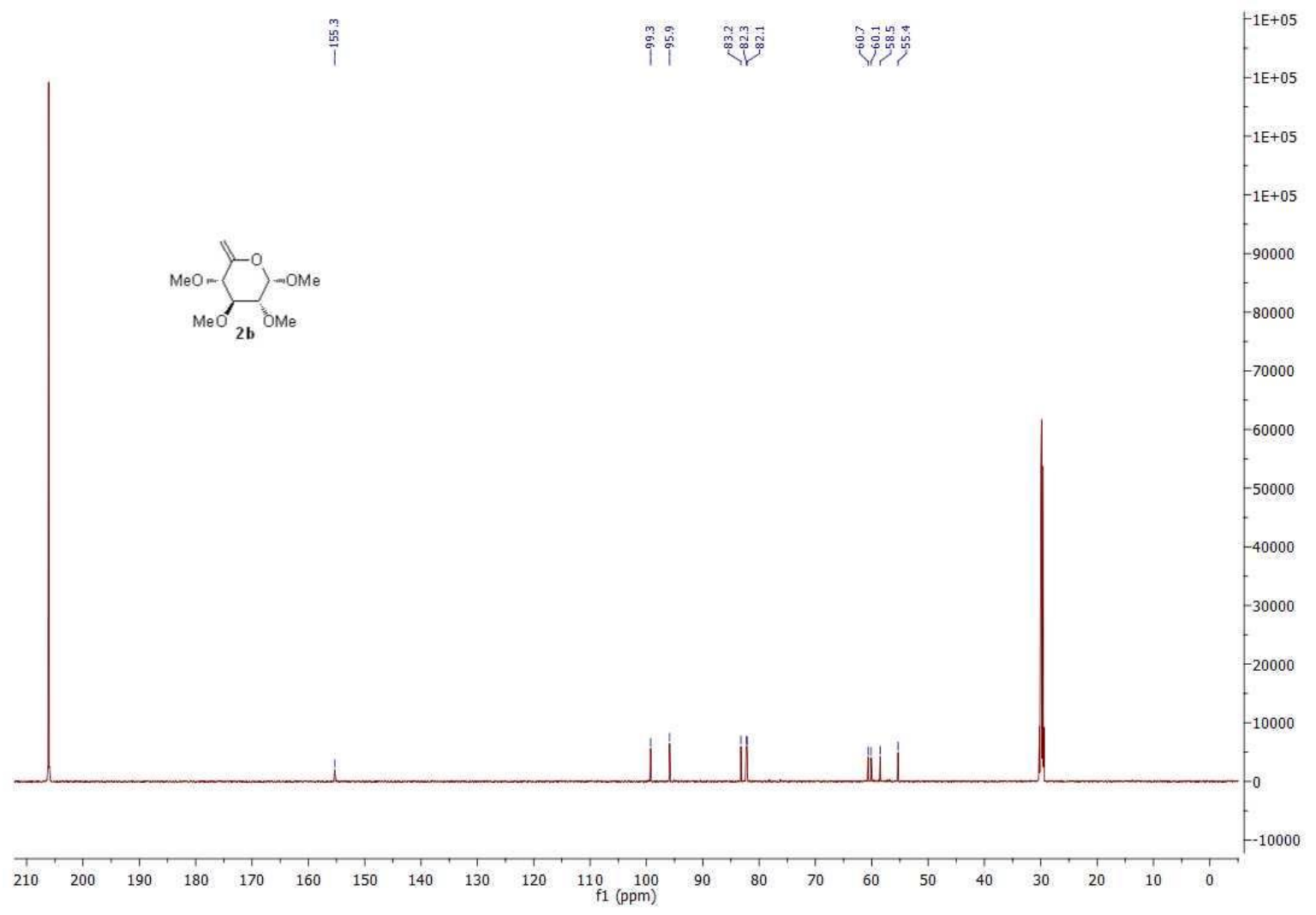

${ }^{13} \mathrm{C}-\mathrm{NMR}$ Spectrum of Compound $\mathbf{2 b}$ (Acetone-d6) 


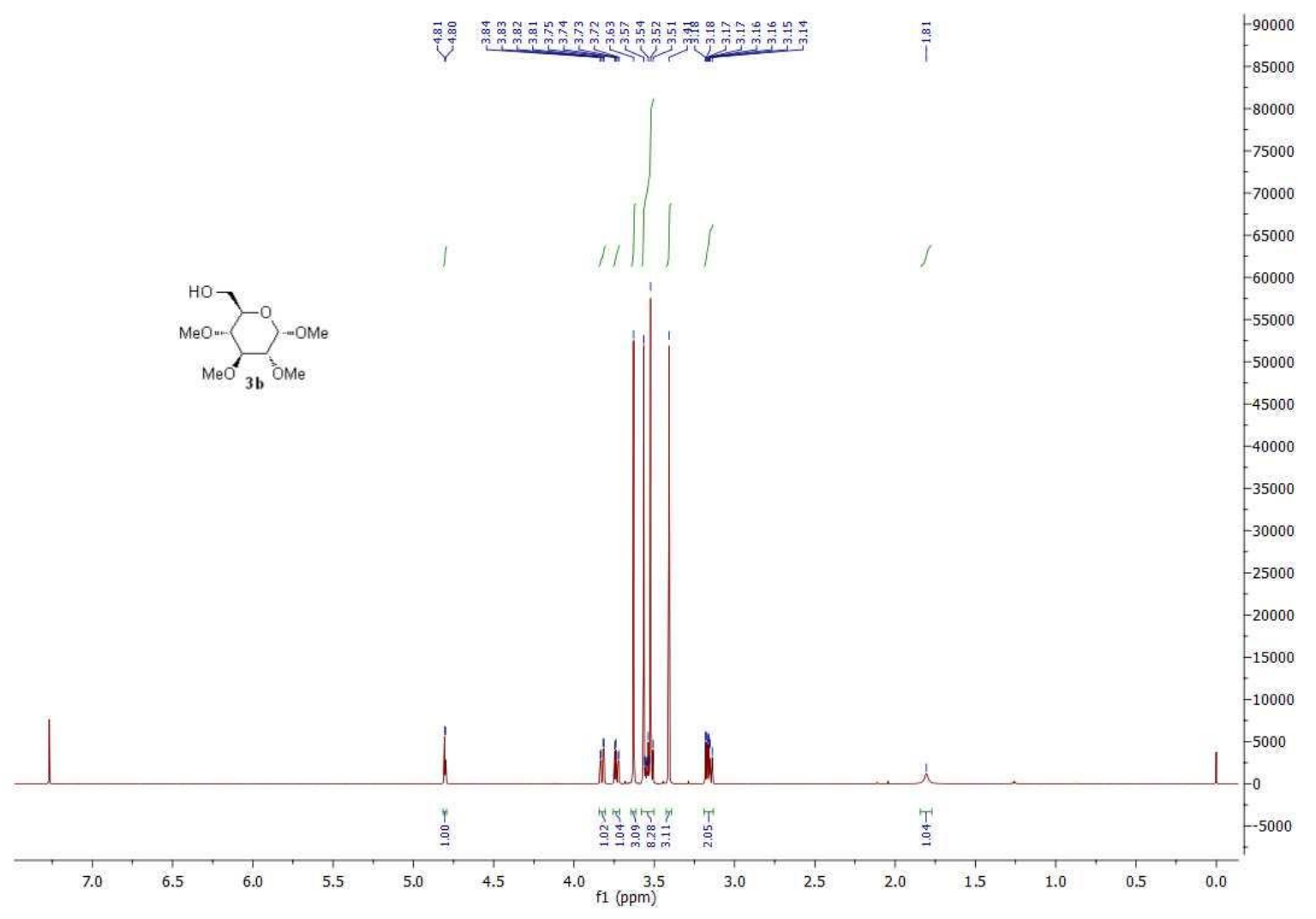

${ }^{1} \mathrm{H}-\mathrm{NMR}$ Spectrum of Compound $\mathbf{3 b}\left(\mathrm{CDCl}_{3}\right)$

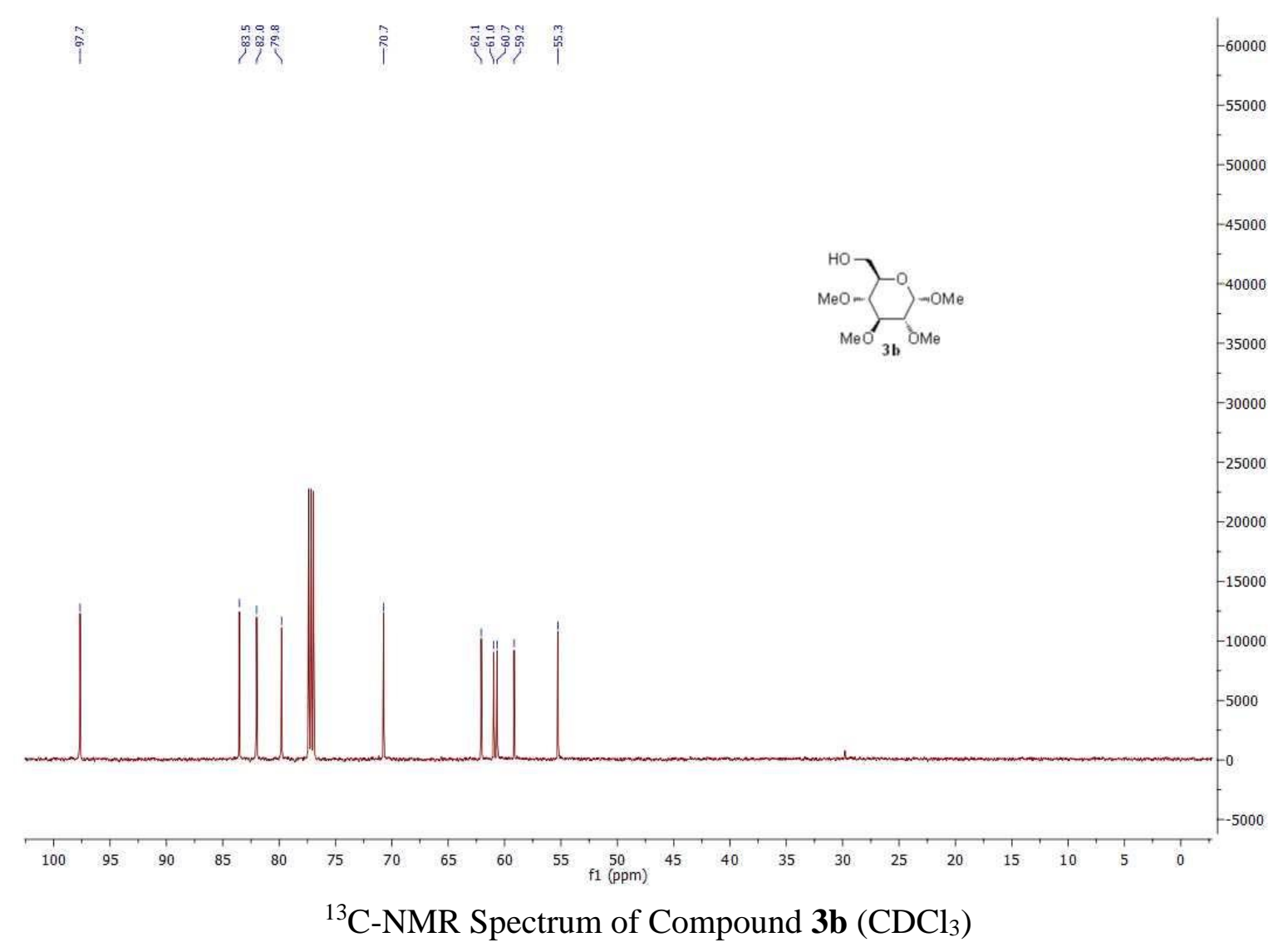




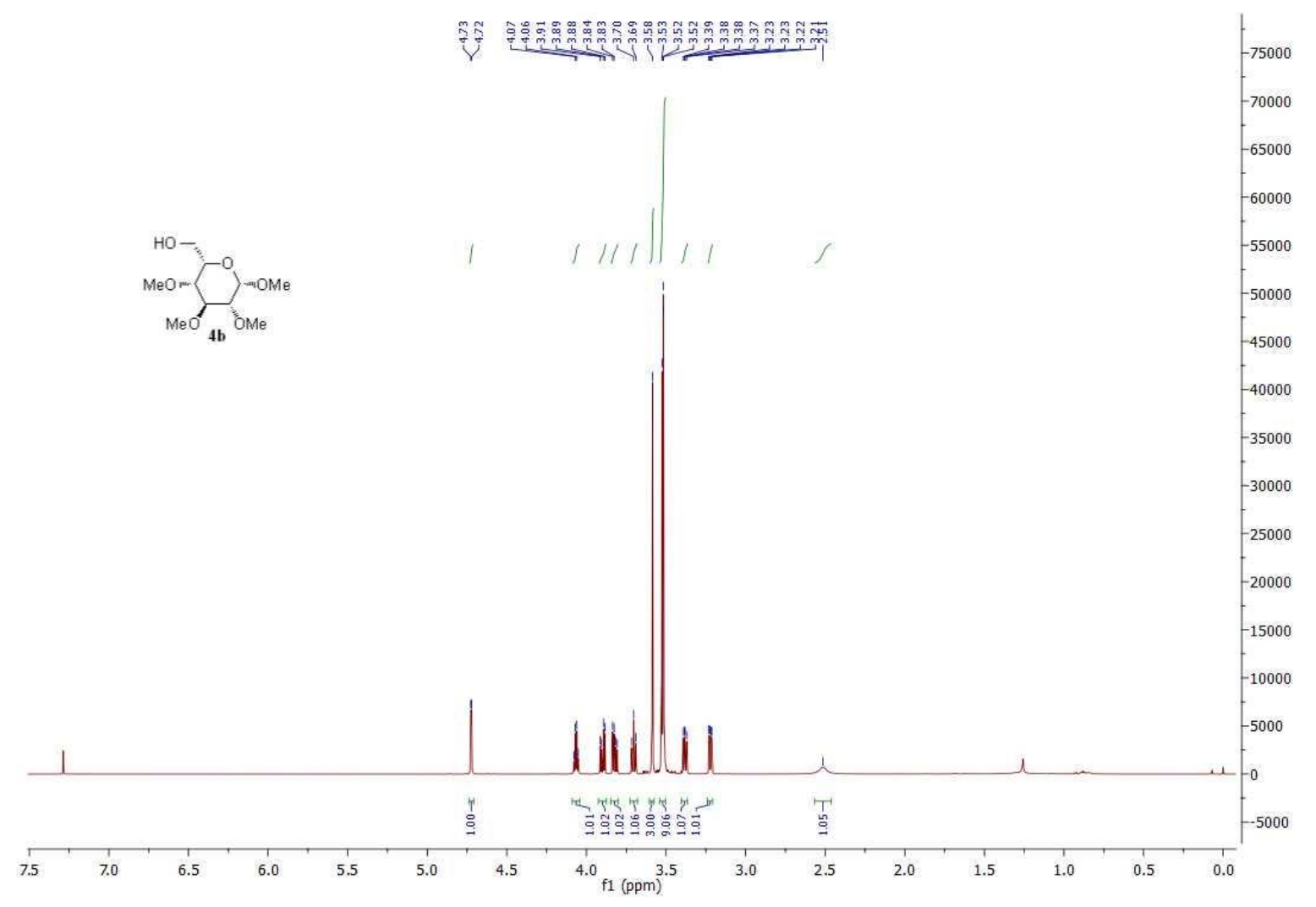

${ }^{1} \mathrm{H}-\mathrm{NMR}$ Spectrum of Compound $\mathbf{4 b}\left(\mathrm{CDCl}_{3}\right)$

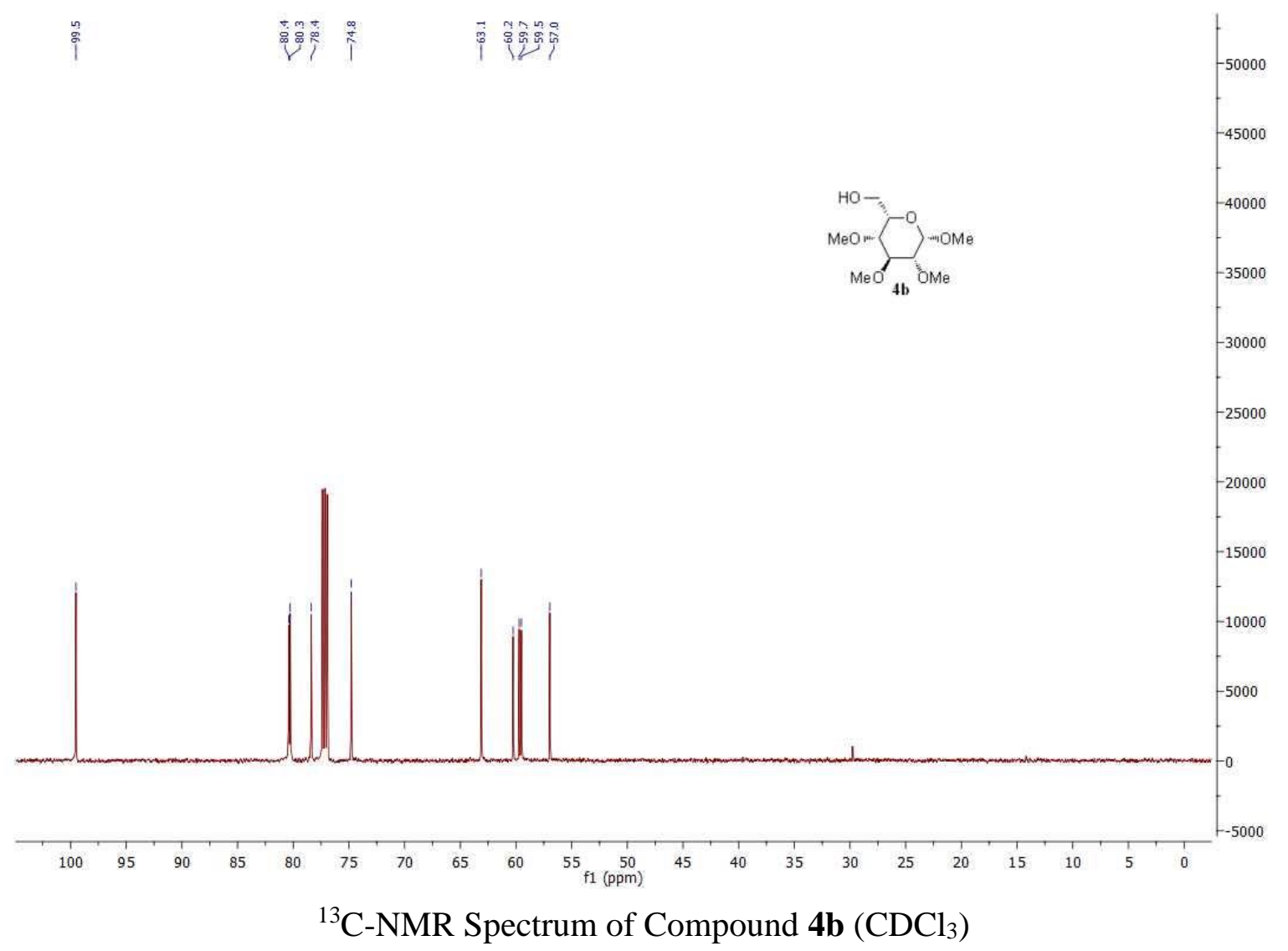




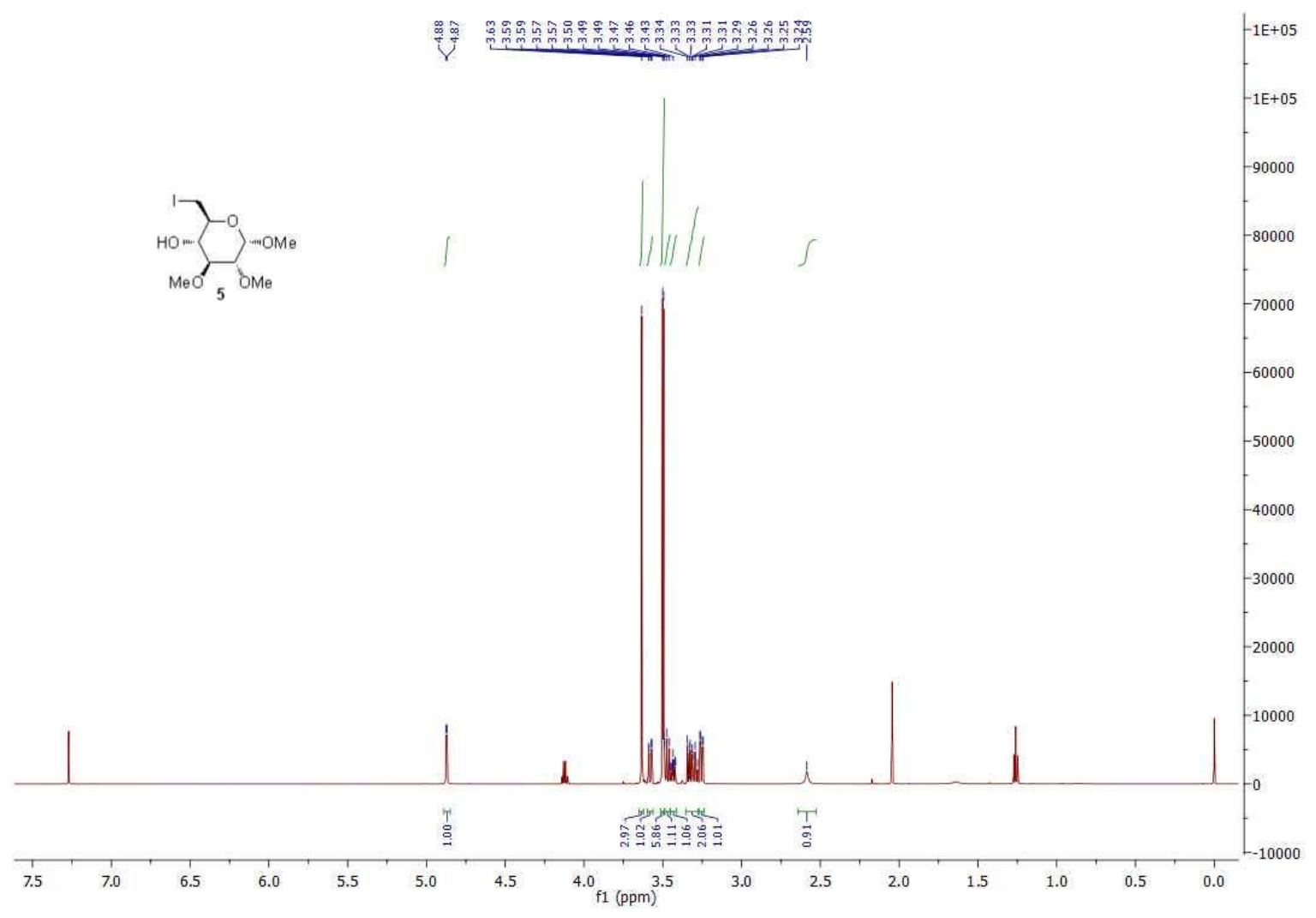

${ }^{1} \mathrm{H}-\mathrm{NMR}$ Spectrum of Compound $\mathbf{5}\left(\mathrm{CDCl}_{3}\right)$

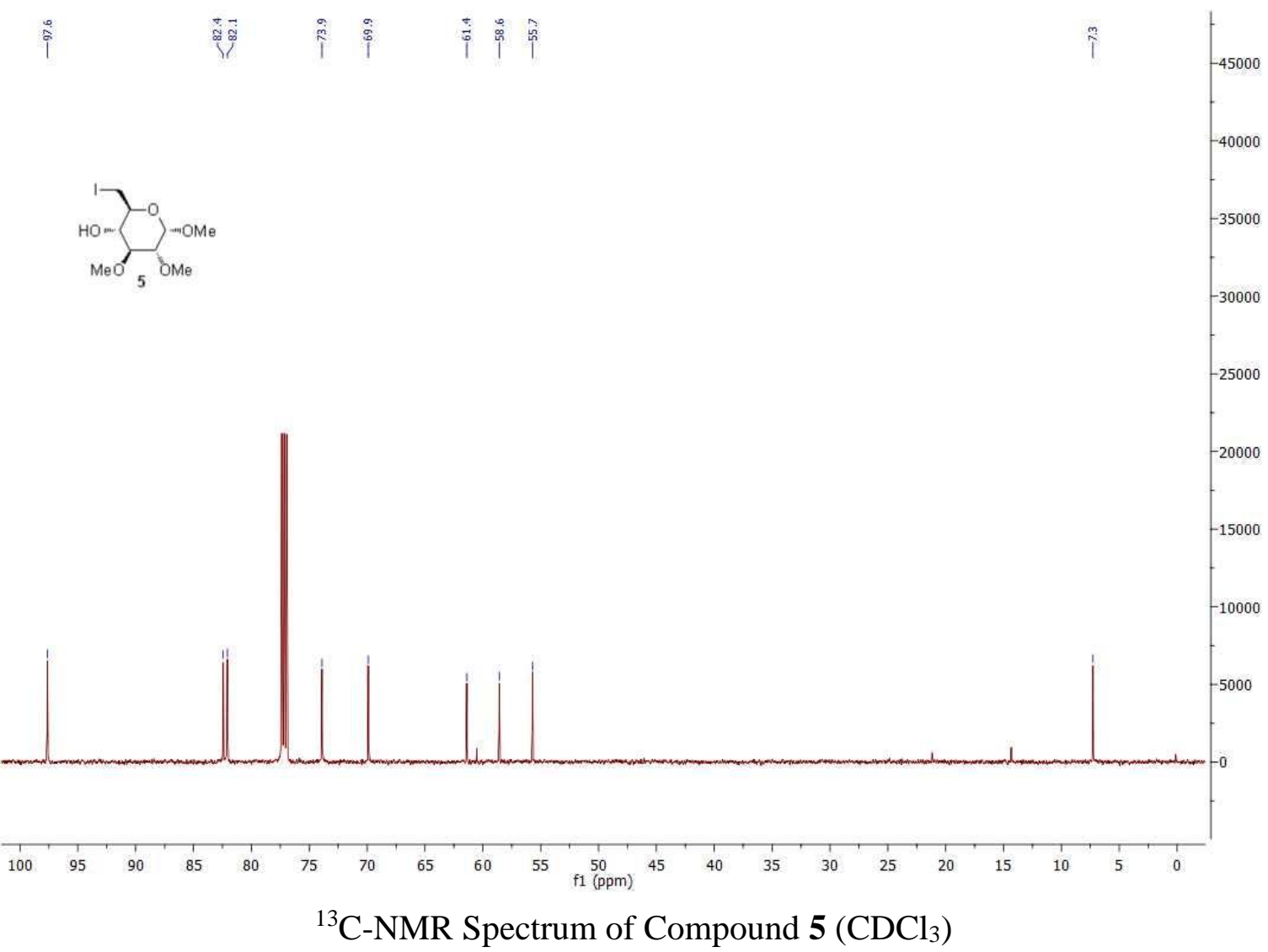




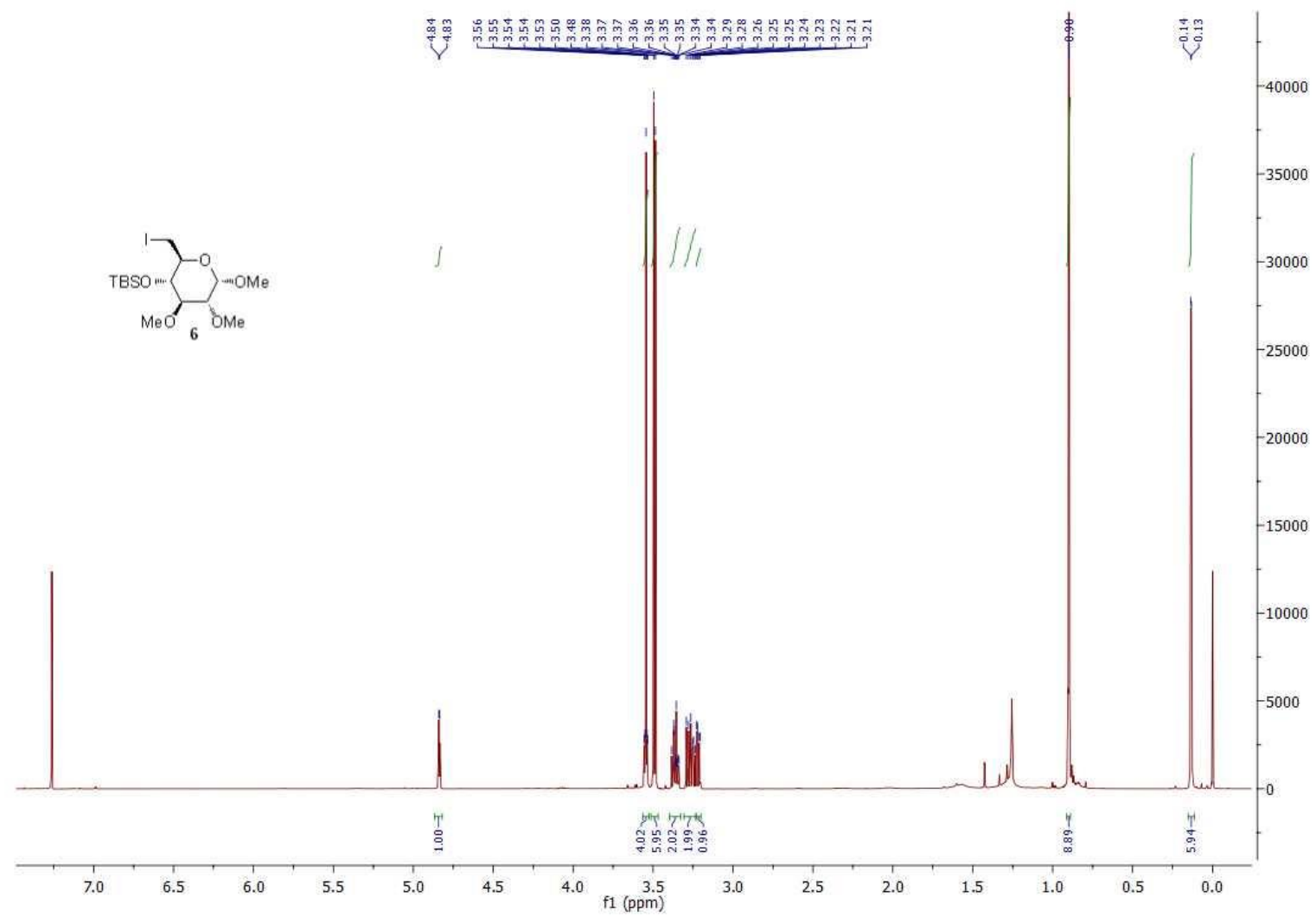

${ }^{1} \mathrm{H}-\mathrm{NMR}$ Spectrum of Compound $\mathbf{6}\left(\mathrm{CDCl}_{3}\right)$

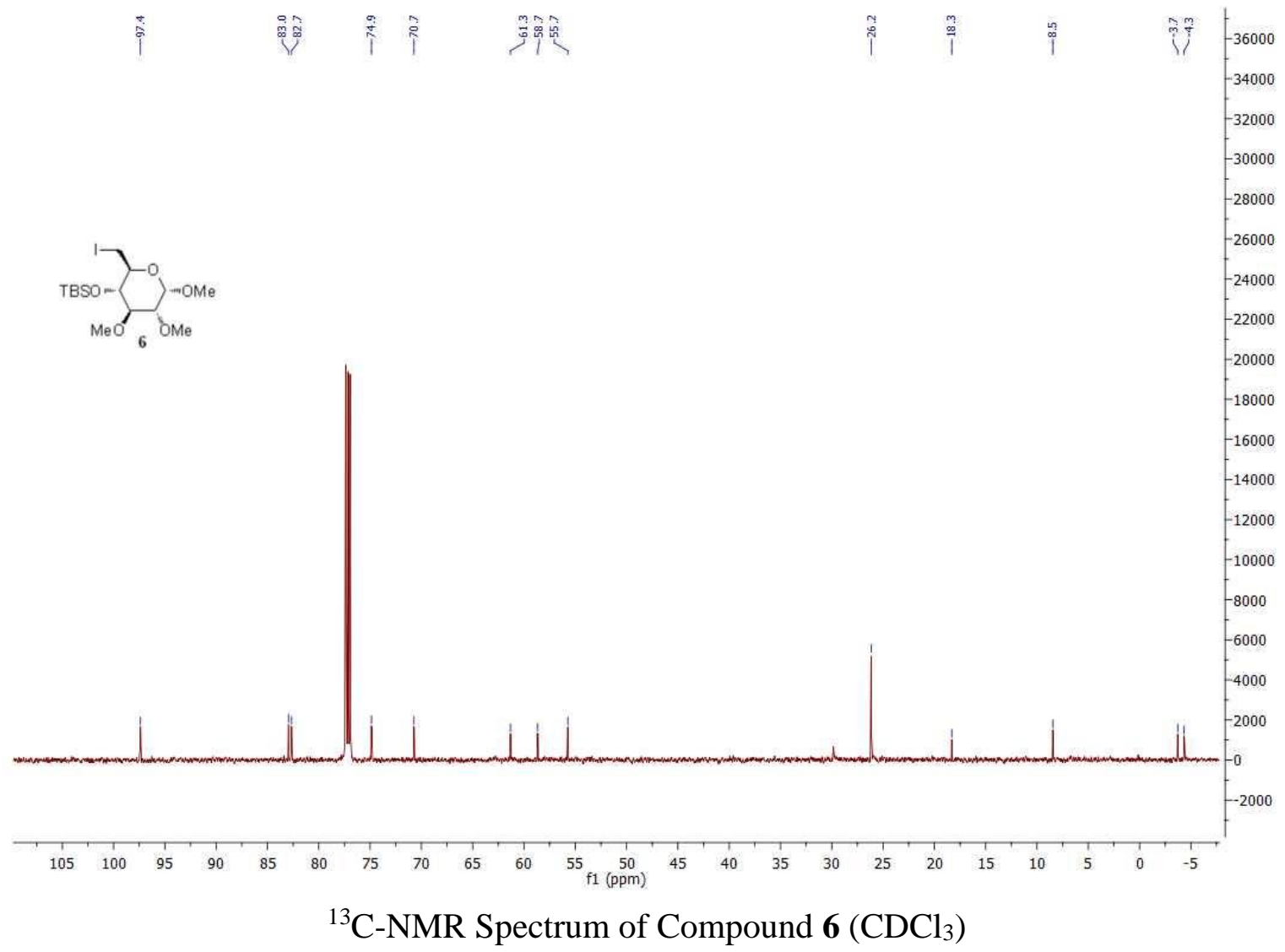




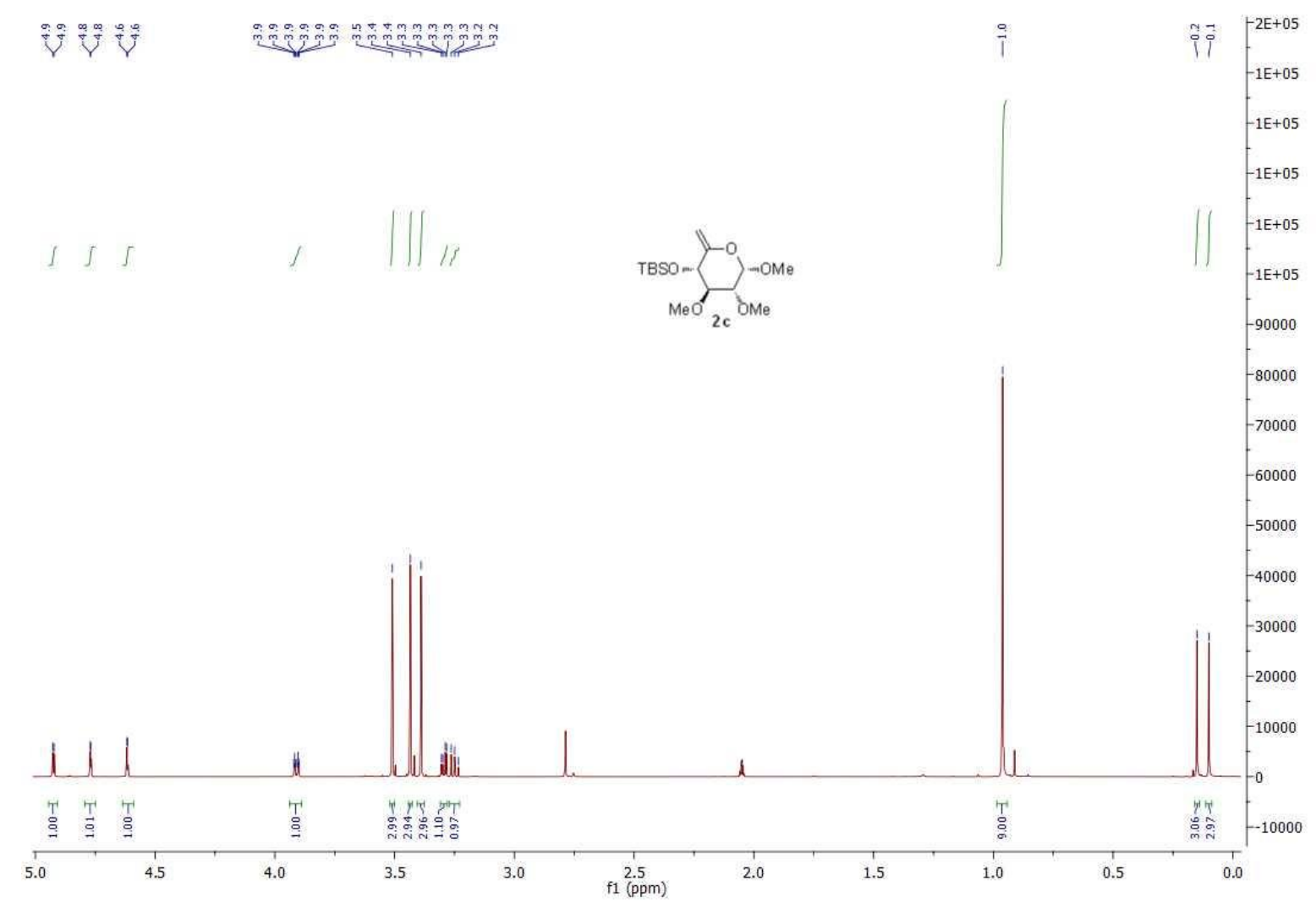

${ }^{1} \mathrm{H}-\mathrm{NMR}$ Spectrum of Compound 2c (Acetone-d6)

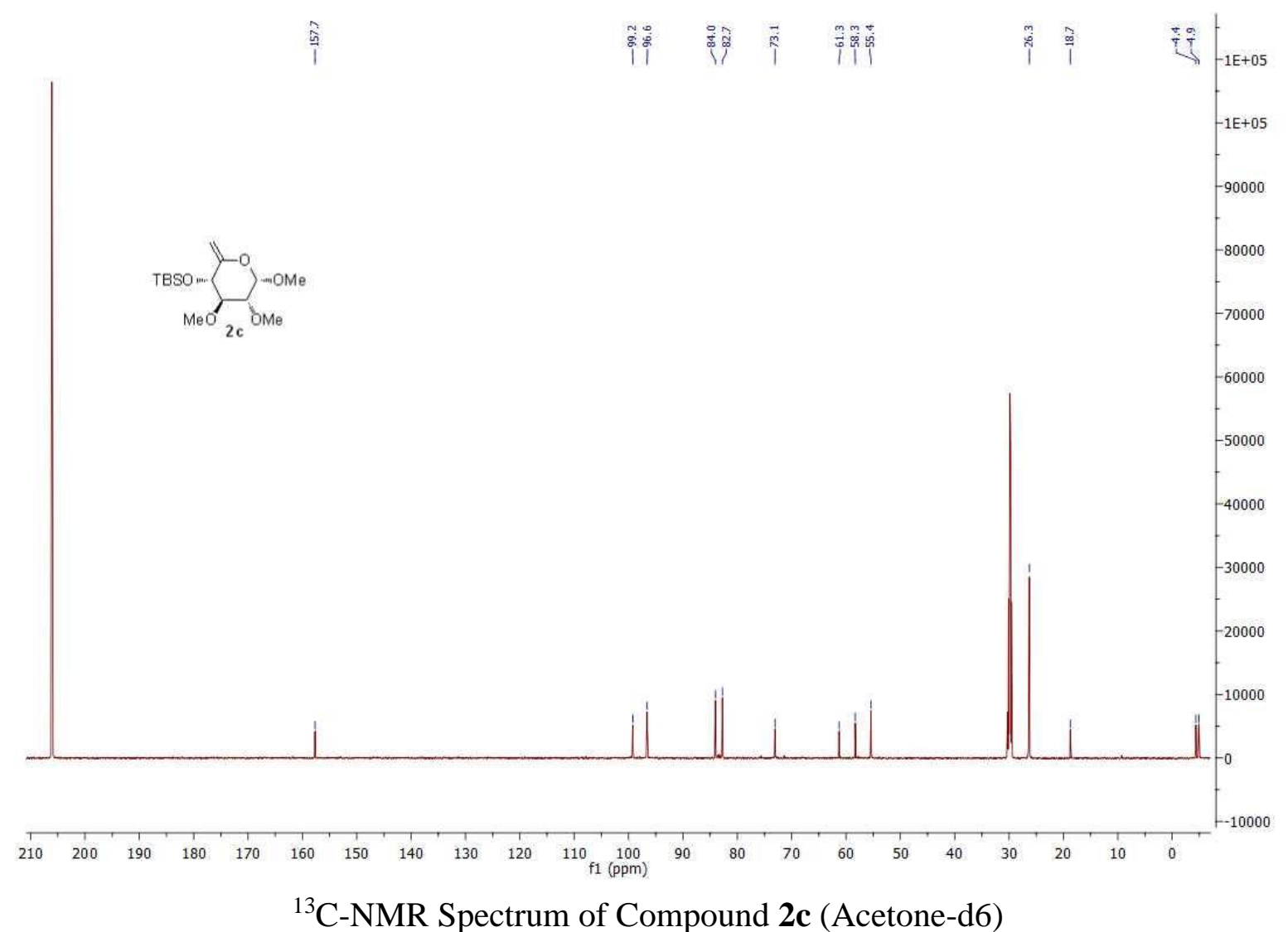




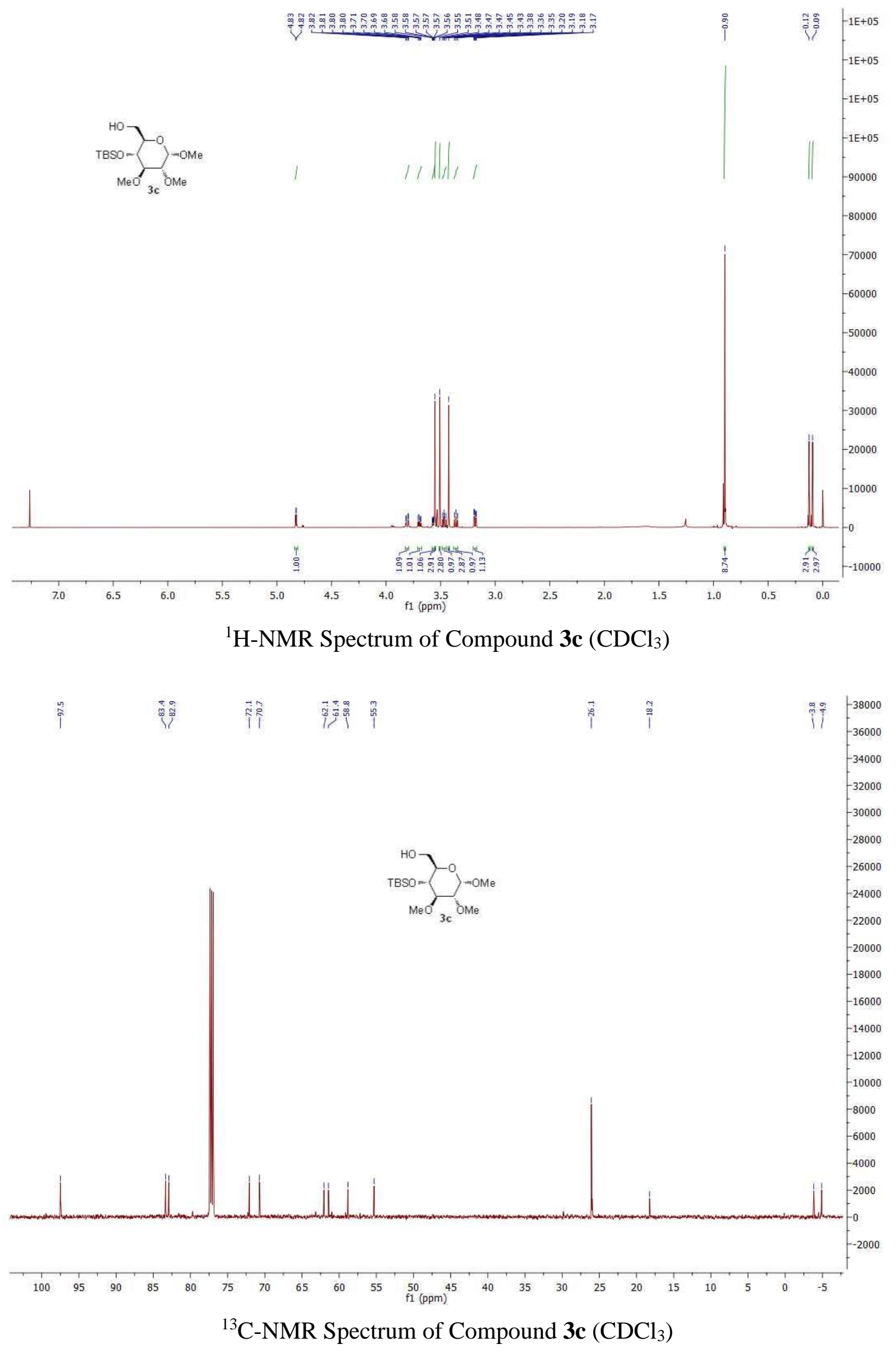




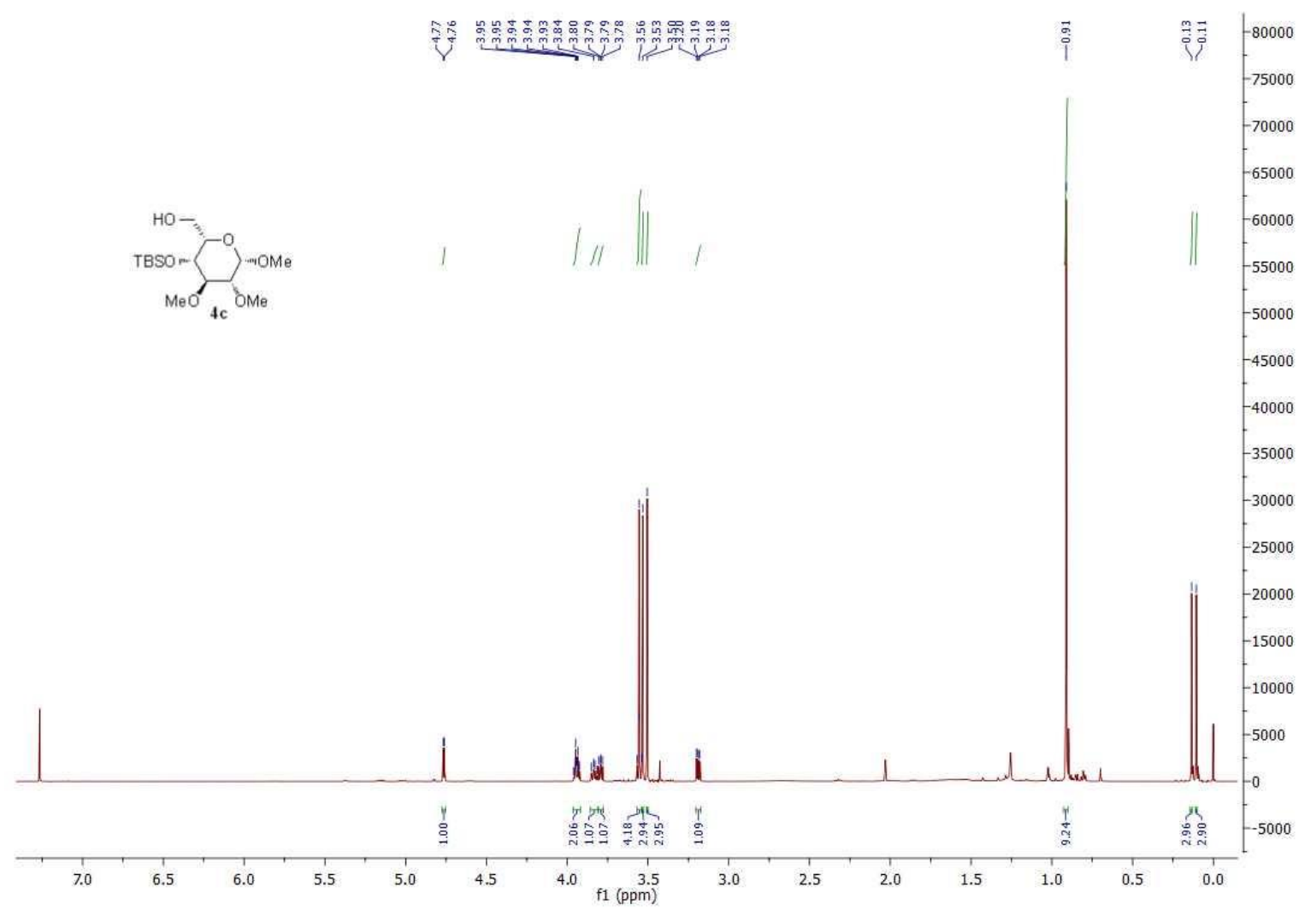

${ }^{1} \mathrm{H}-\mathrm{NMR}$ Spectrum of Compound $\mathbf{4} \mathbf{c}\left(\mathrm{CDCl}_{3}\right)$

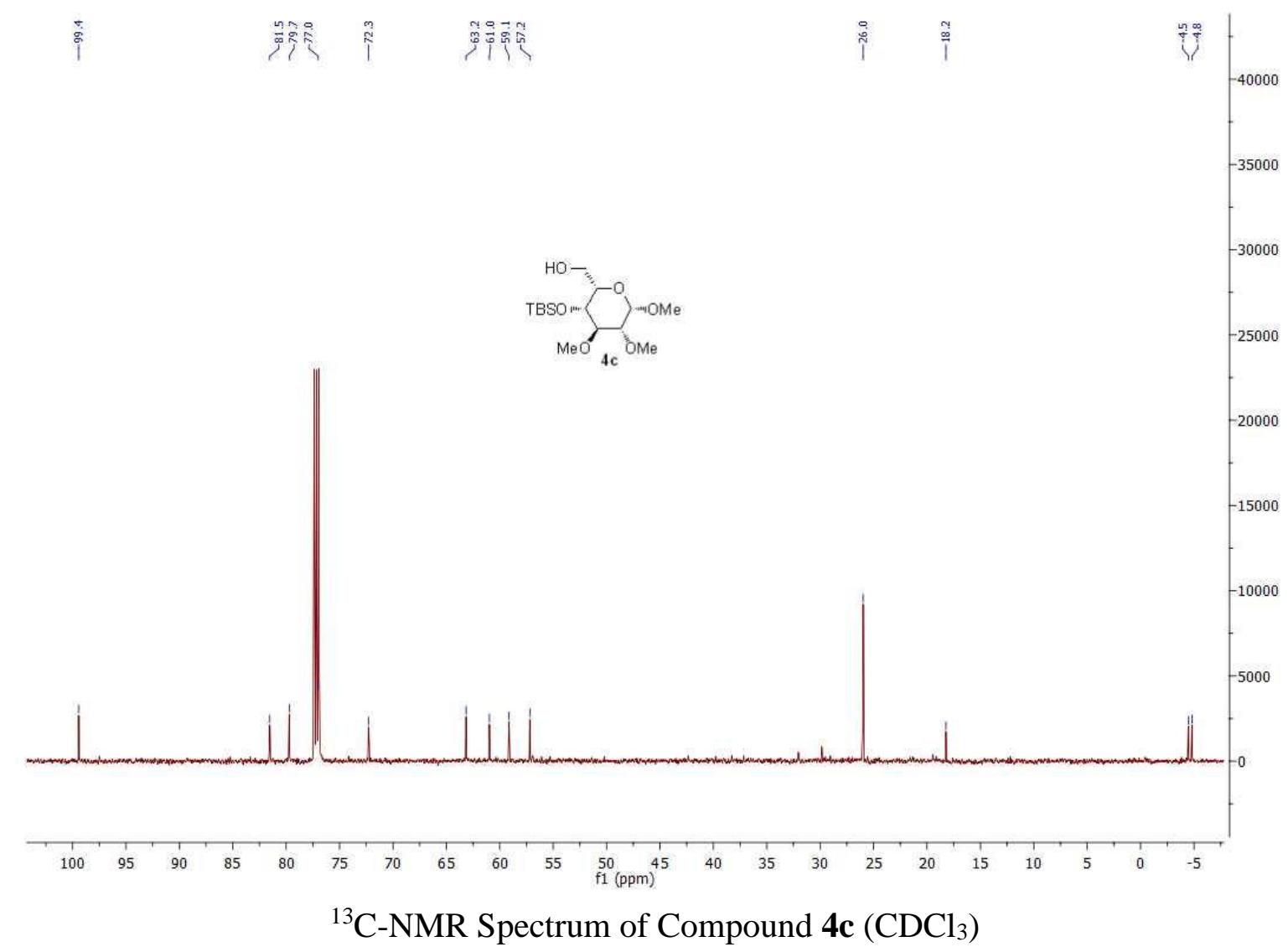




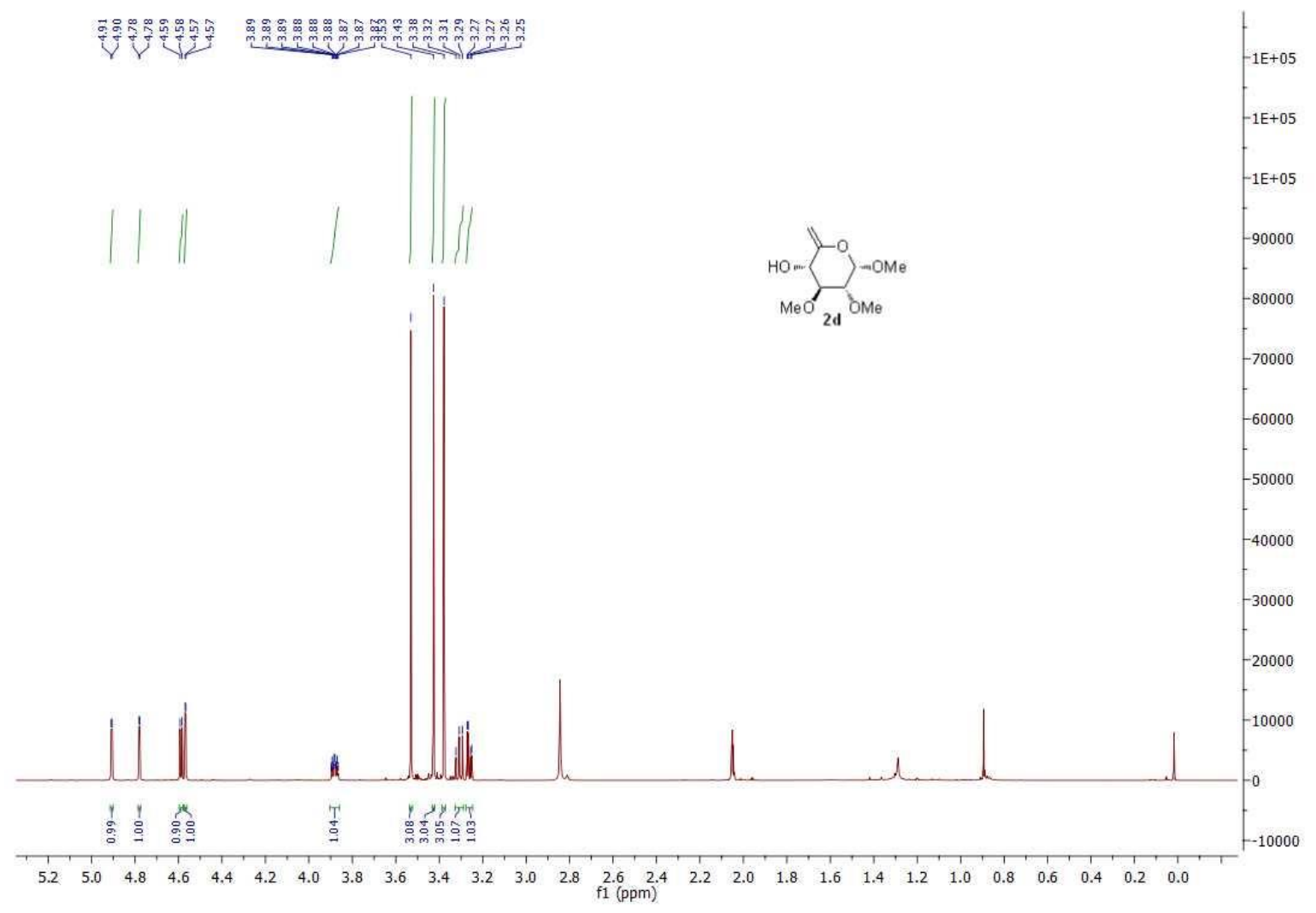

${ }^{1}$ H-NMR Spectrum of Compound 2d (Acetone-d6)

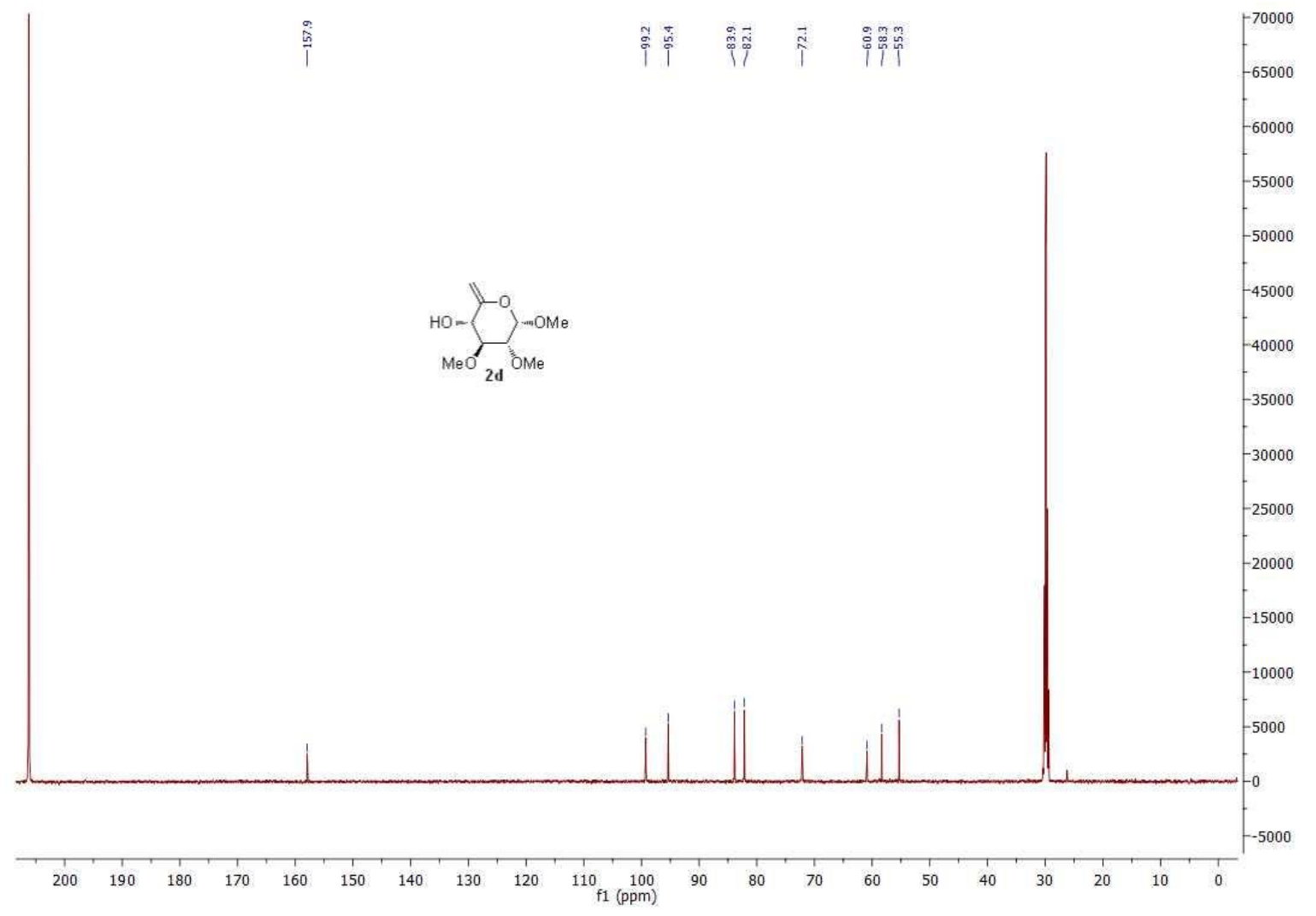

${ }^{13}$ C-NMR Spectrum of Compound 2d (Acetone-d6) 


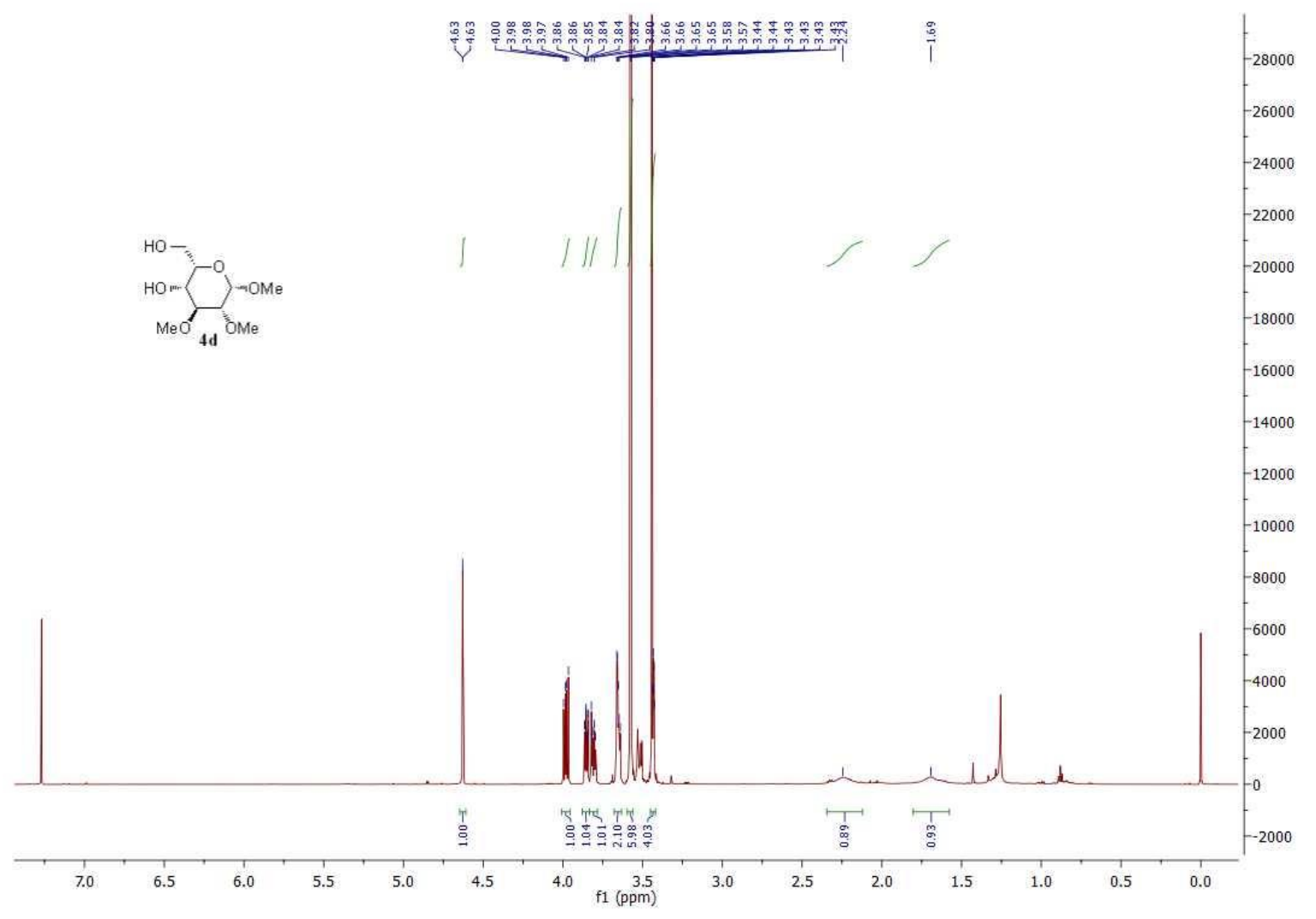

${ }^{1} \mathrm{H}-\mathrm{NMR}$ Spectrum of Compound $\mathbf{4 d}\left(\mathrm{CDCl}_{3}\right)$

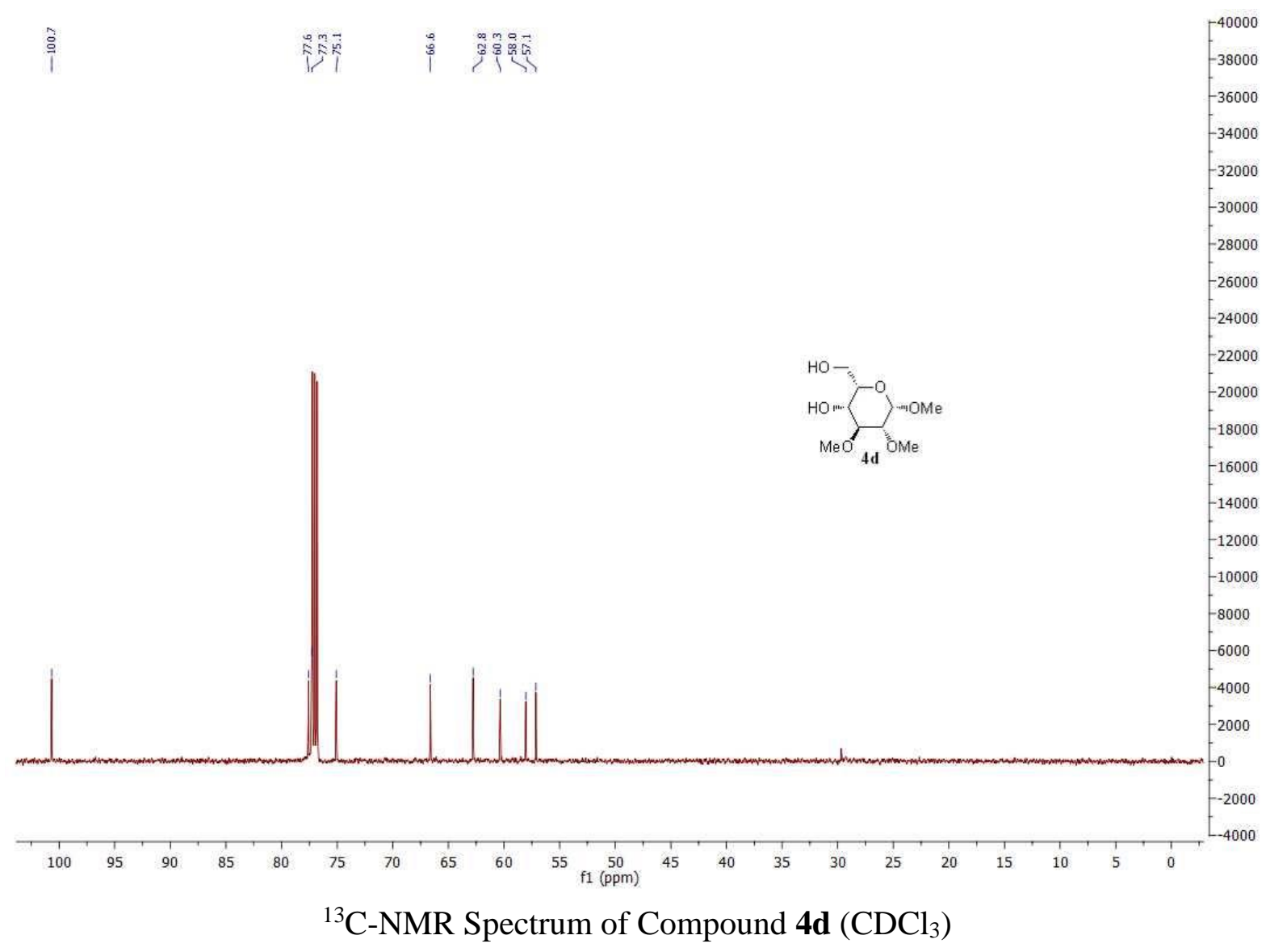




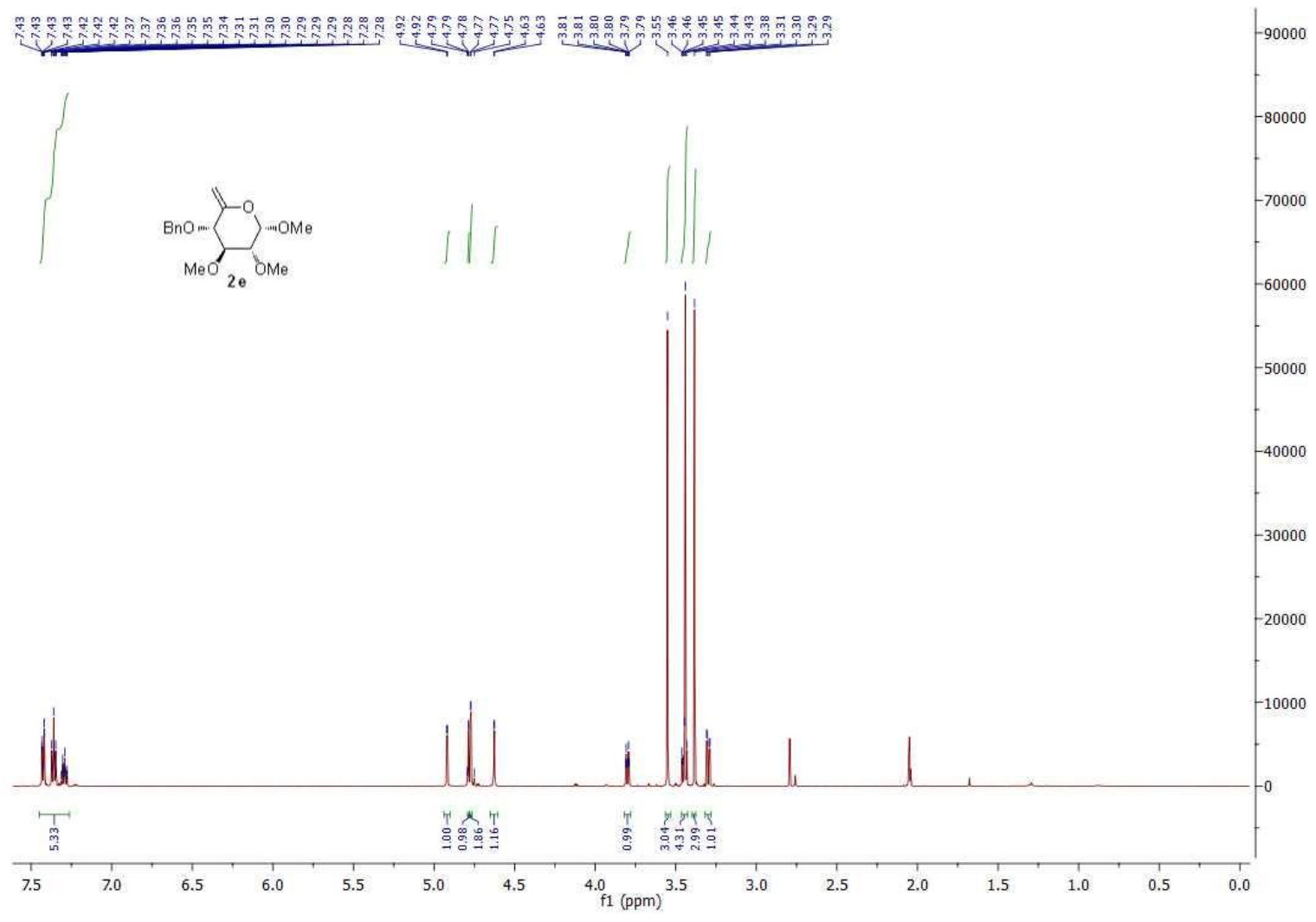

${ }^{1} \mathrm{H}-\mathrm{NMR}$ Spectrum of Compound $\mathbf{2 e}$ (Acetone-d6)

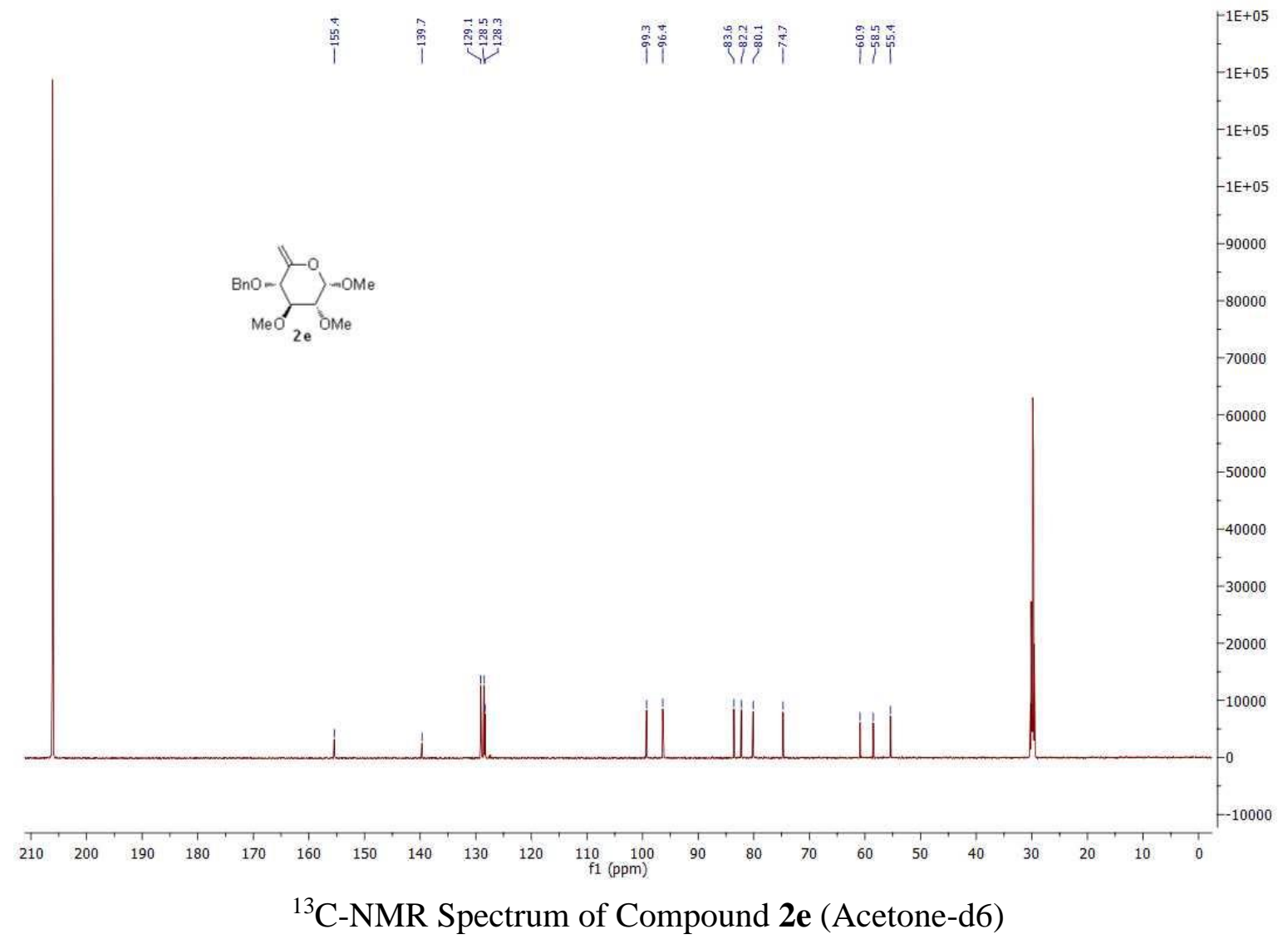




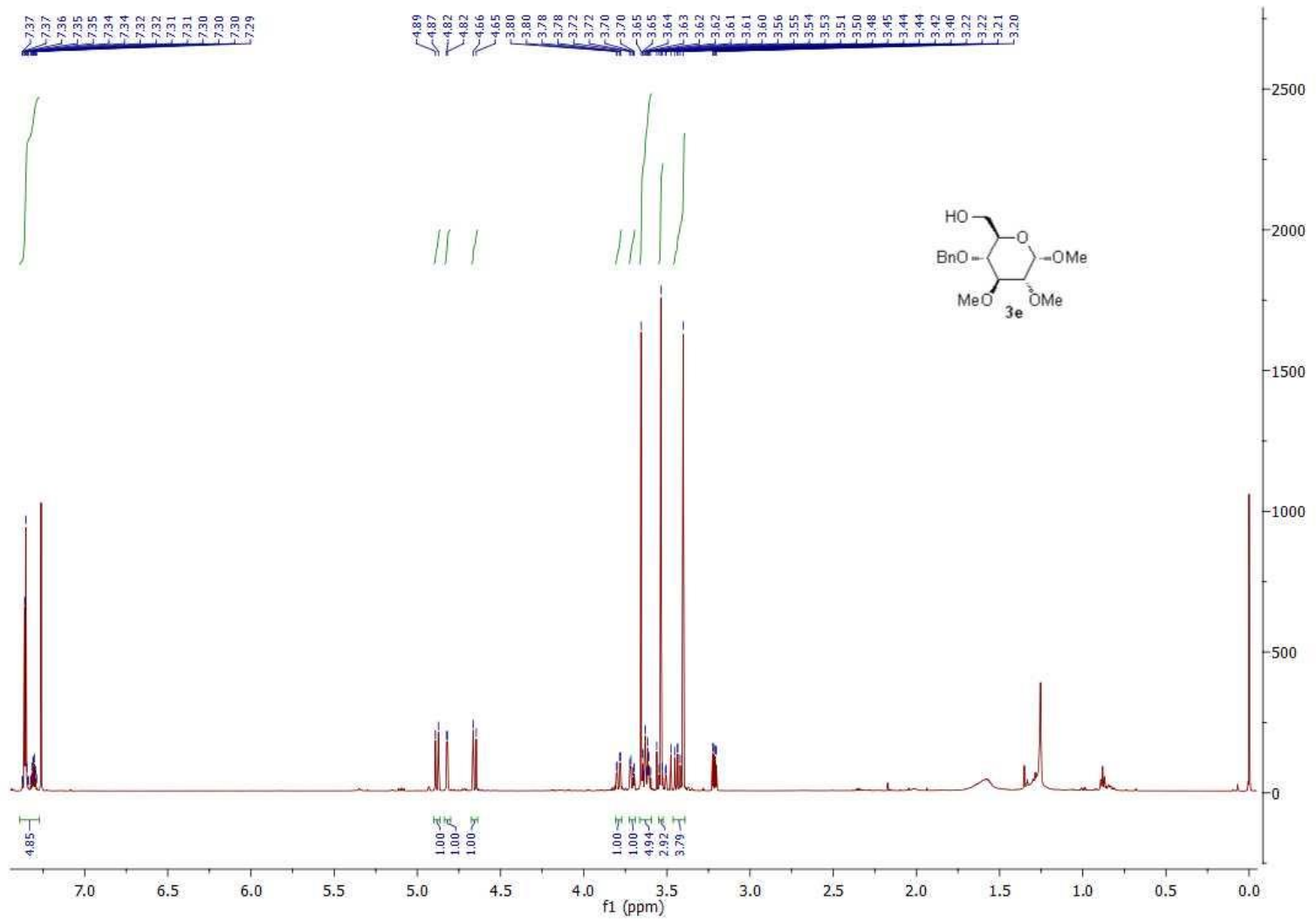

${ }^{1} \mathrm{H}-\mathrm{NMR}$ Spectrum of Compound $\mathbf{3 e}\left(\mathrm{CDCl}_{3}\right)$

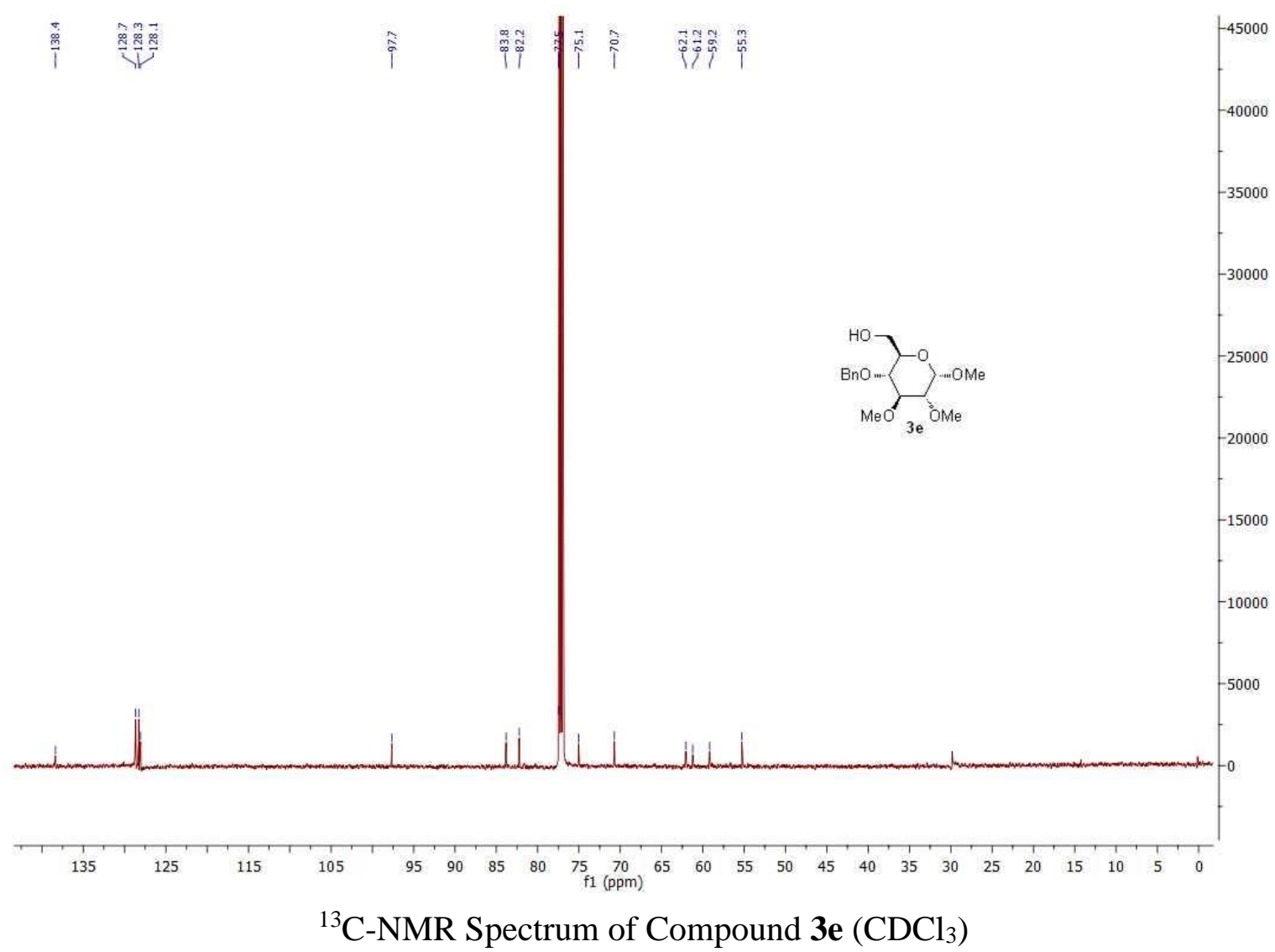




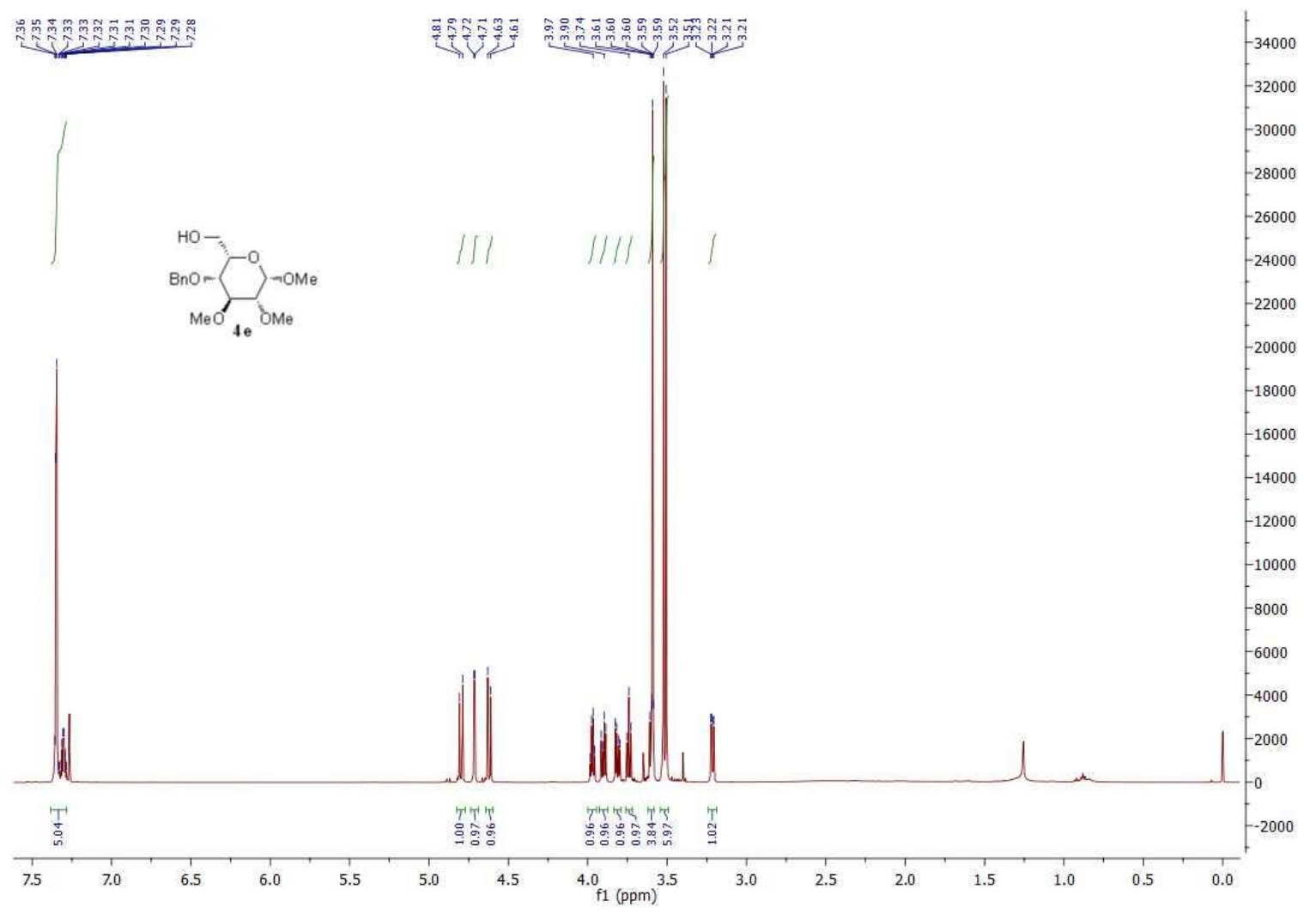

${ }^{1} \mathrm{H}-\mathrm{NMR}$ Spectrum of Compound $\mathbf{4 e}\left(\mathrm{CDCl}_{3}\right)$

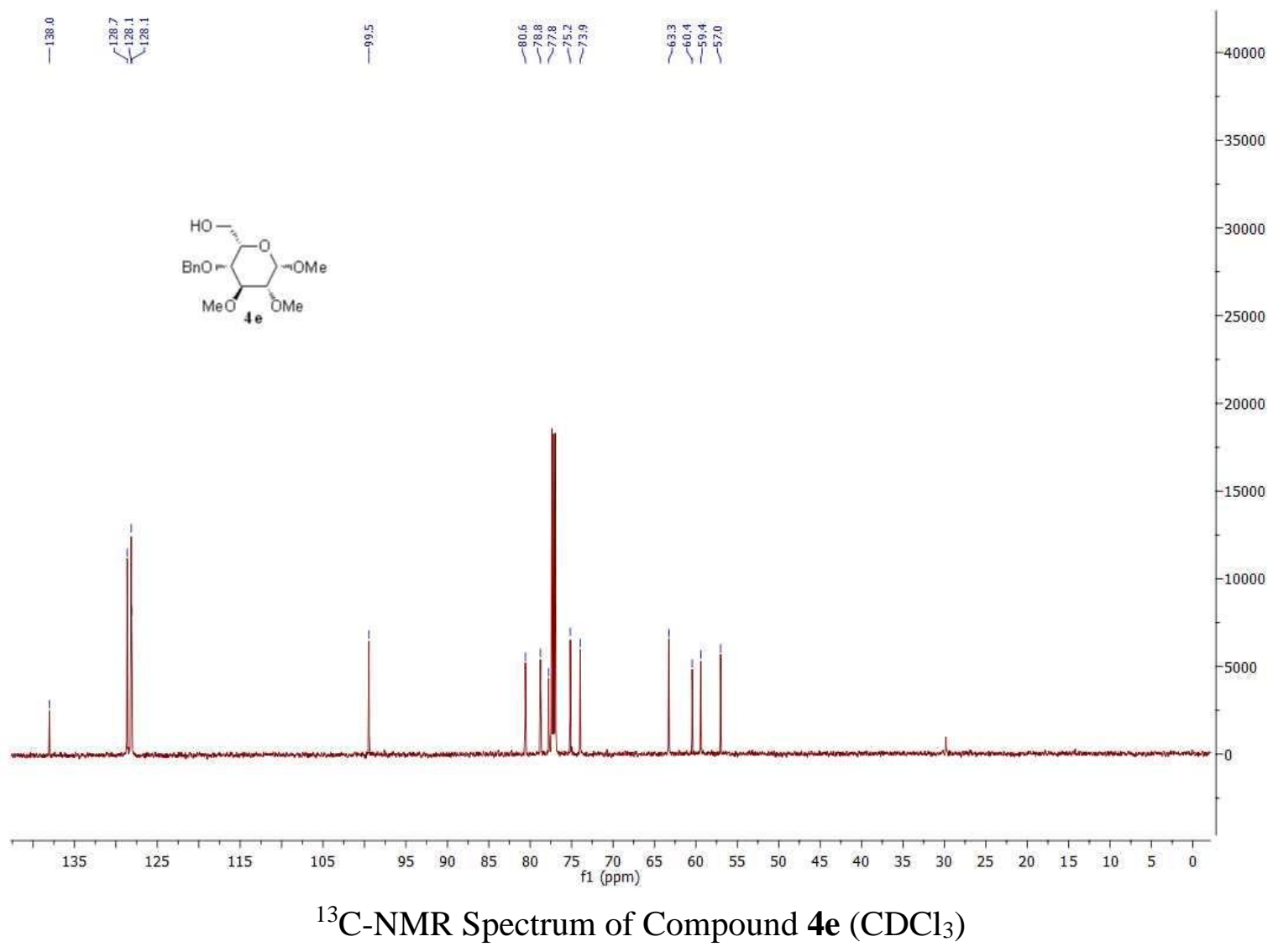




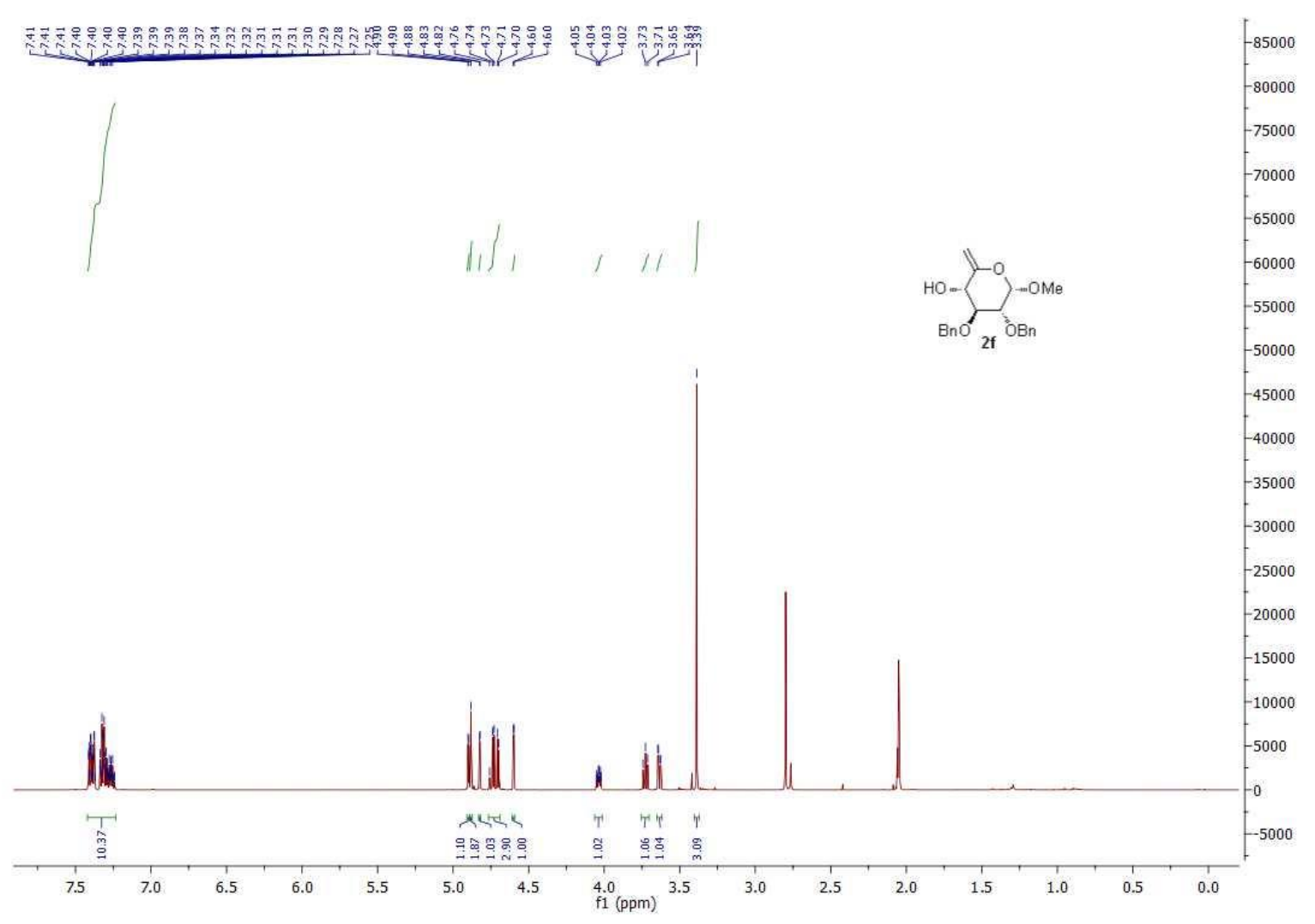

${ }^{1} \mathrm{H}-\mathrm{NMR}$ Spectrum of Compound $\mathbf{2 f}$ (Acetone-d6)

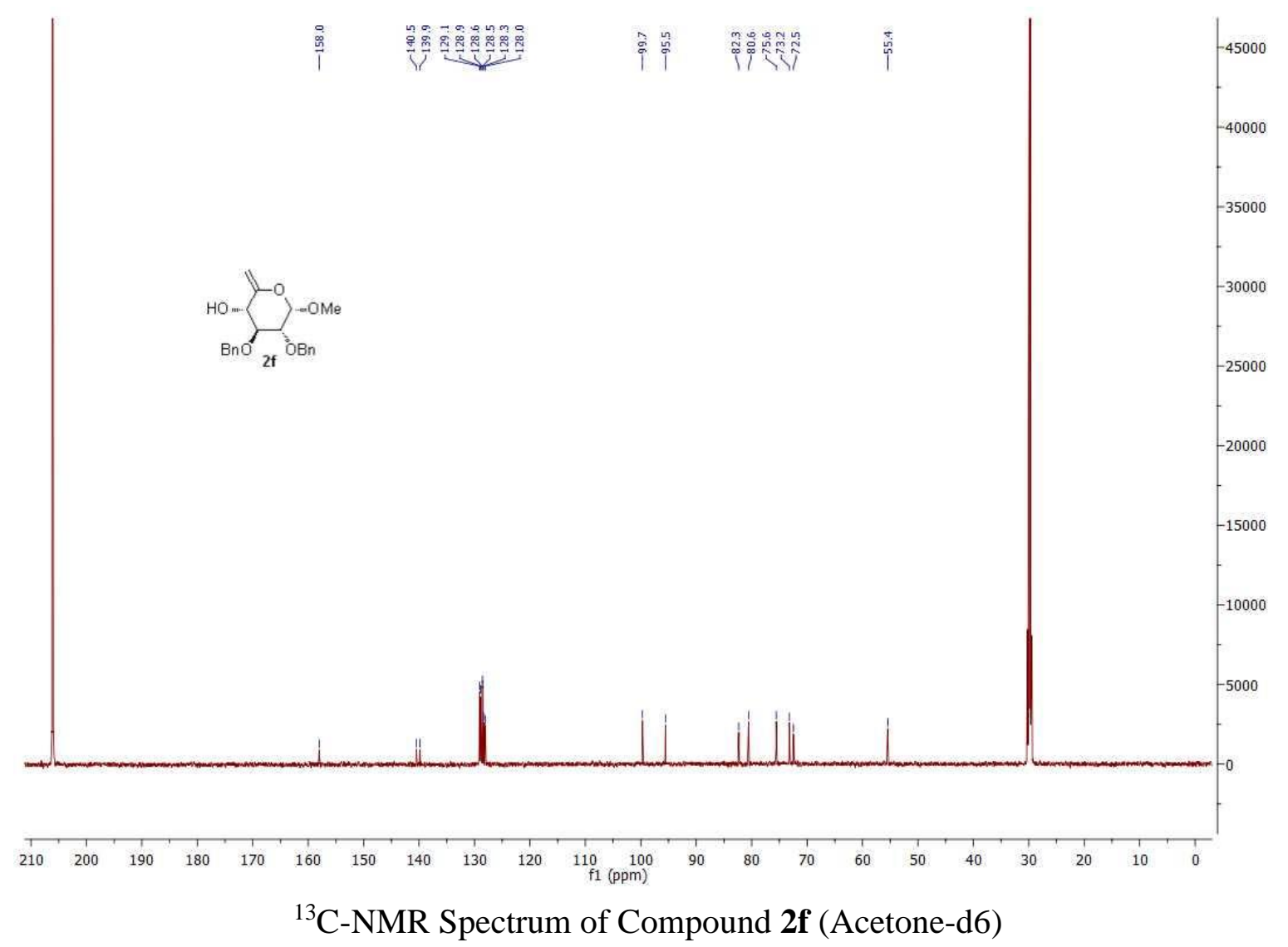




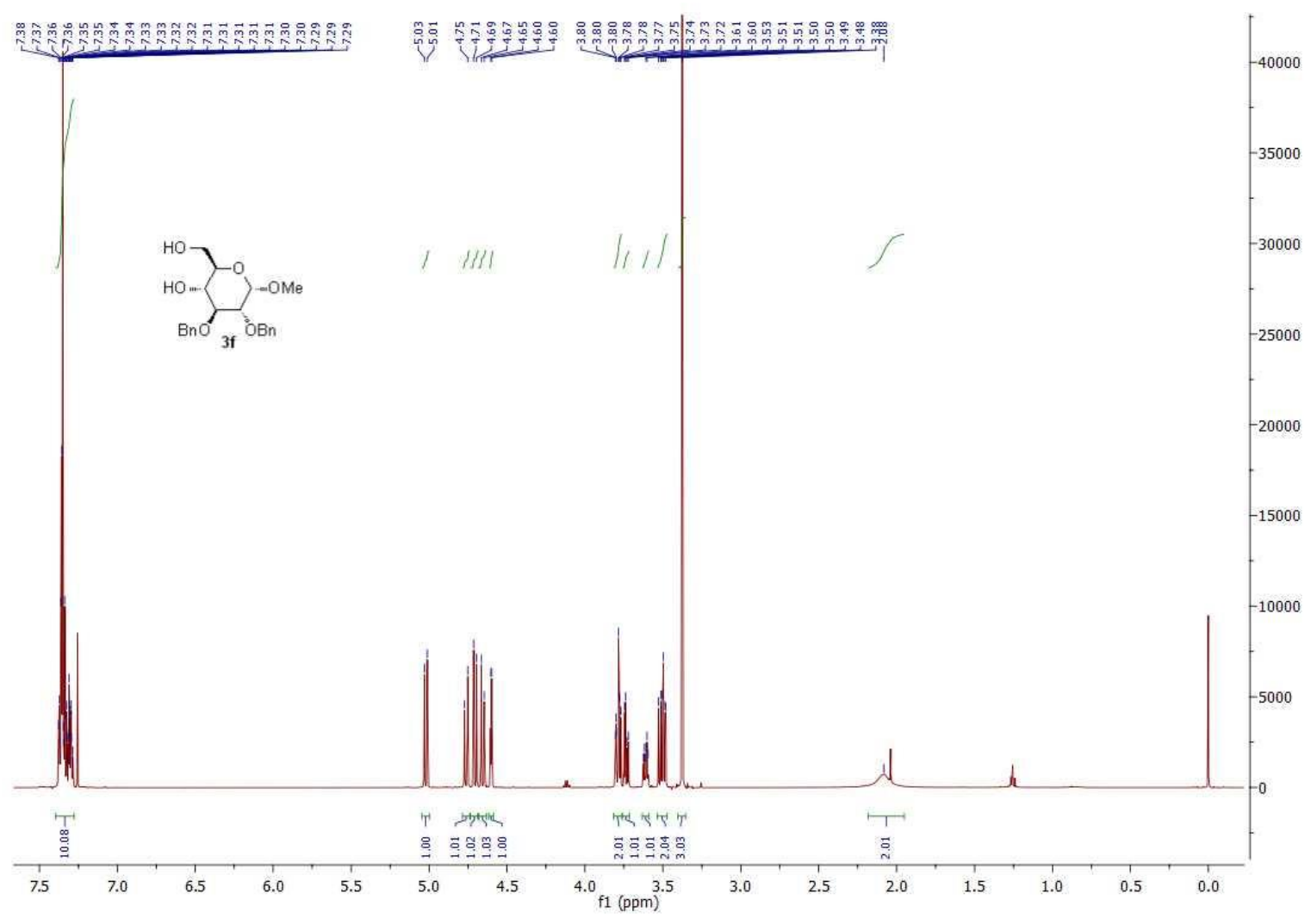

${ }^{1} \mathrm{H}-\mathrm{NMR}$ Spectrum of Compound $\mathbf{3 f}\left(\mathrm{CDCl}_{3}\right)$

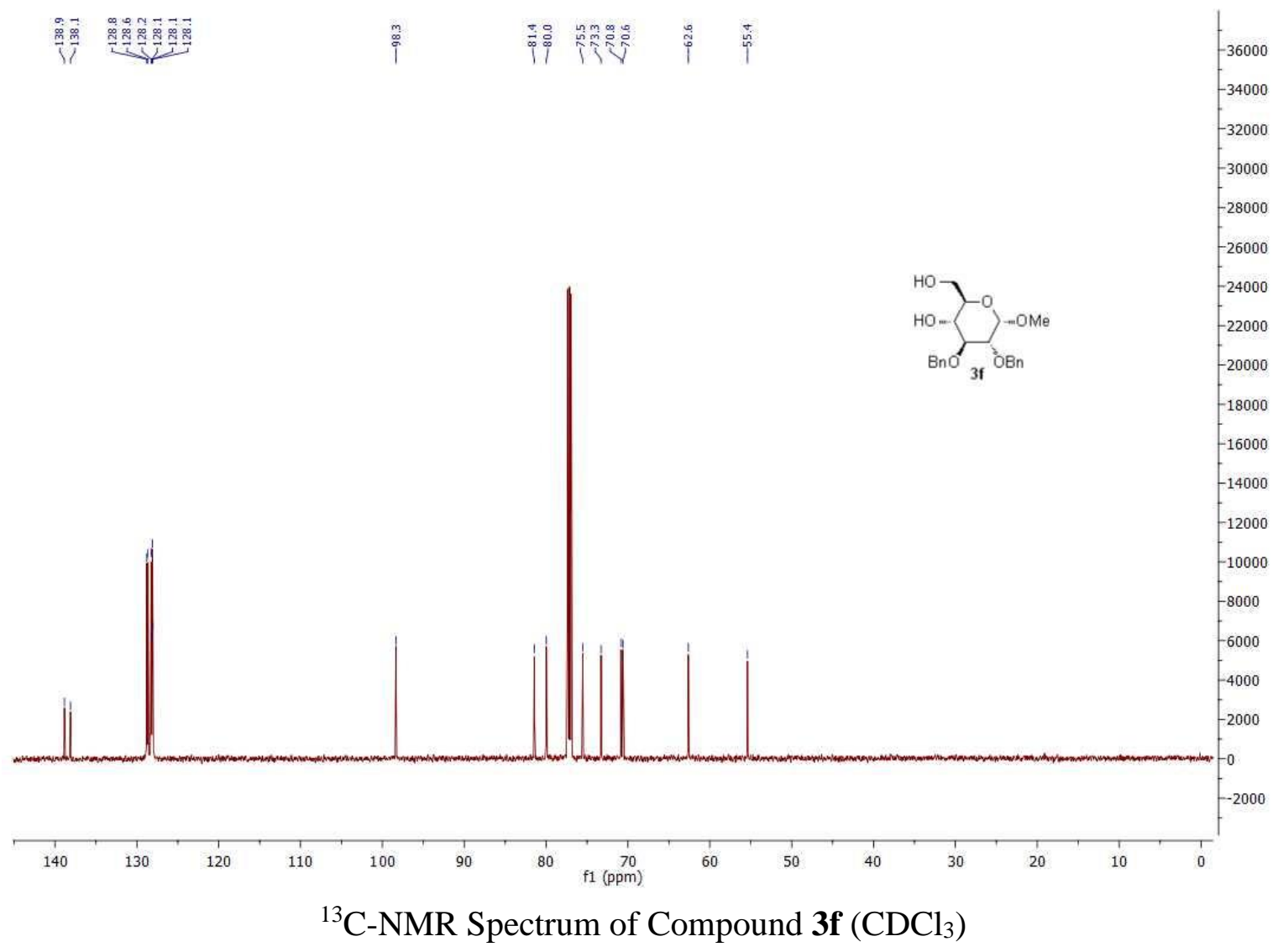




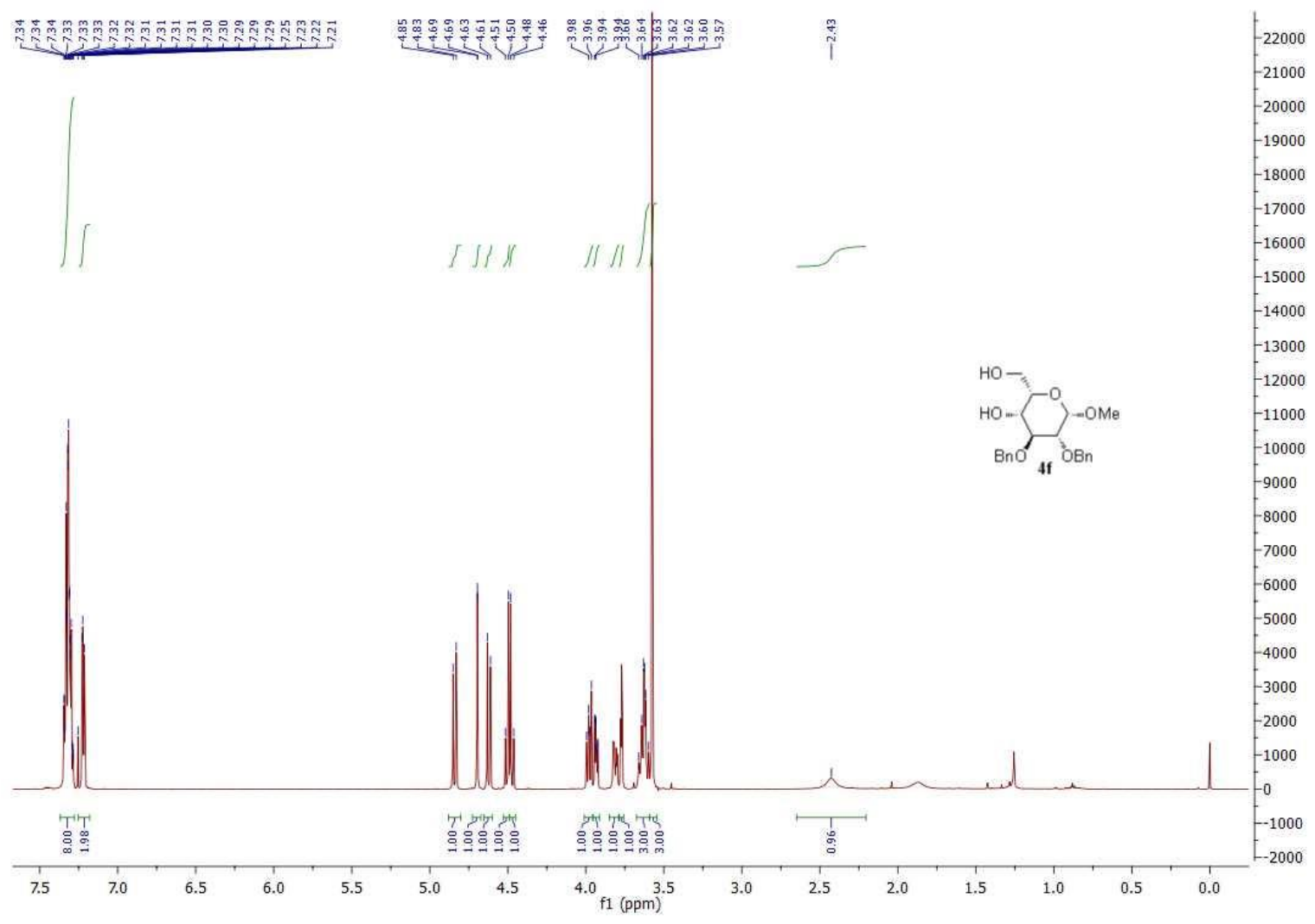

${ }^{1} \mathrm{H}-\mathrm{NMR}$ Spectrum of Compound $\mathbf{4 f}\left(\mathrm{CDCl}_{3}\right)$

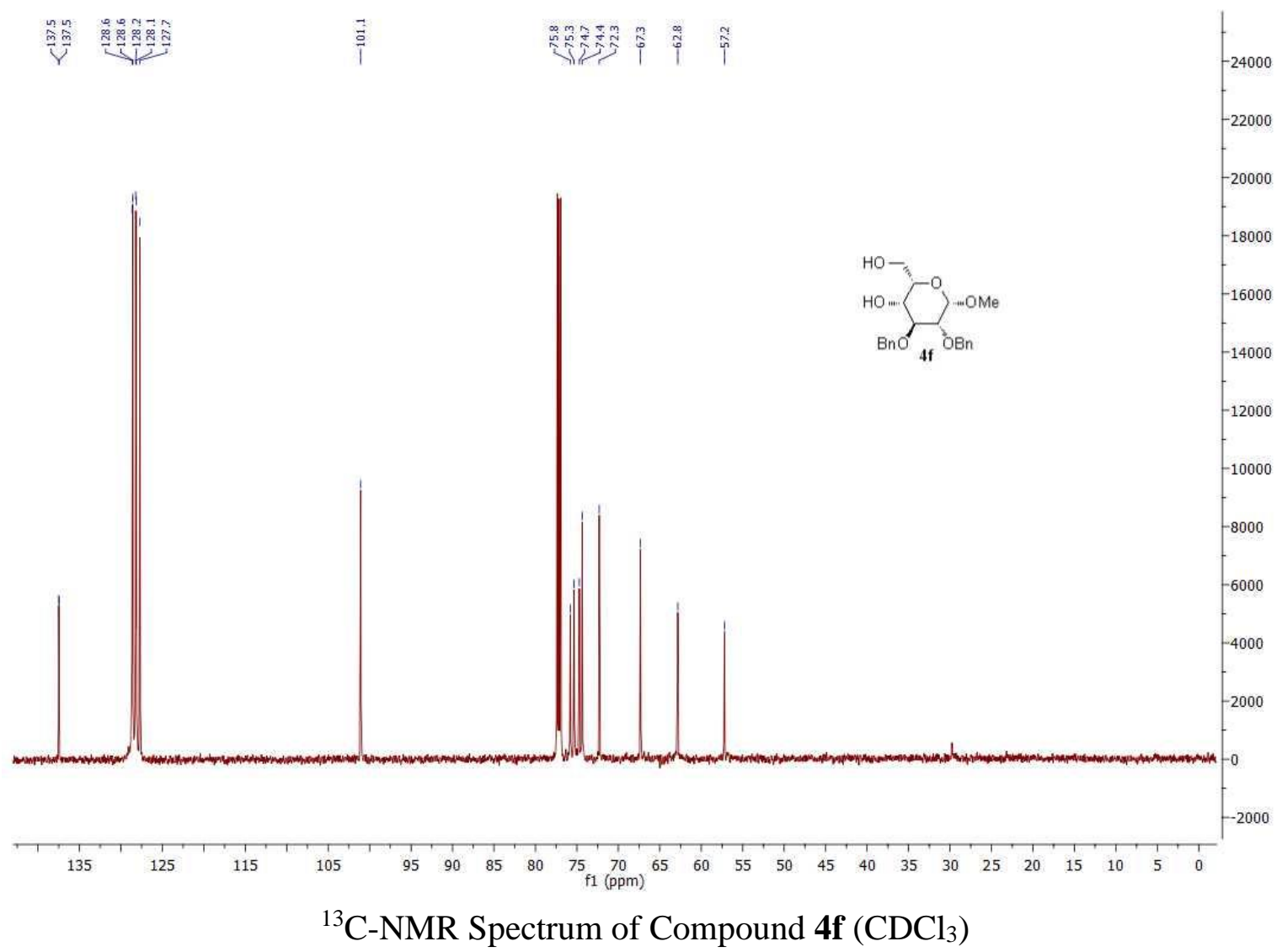




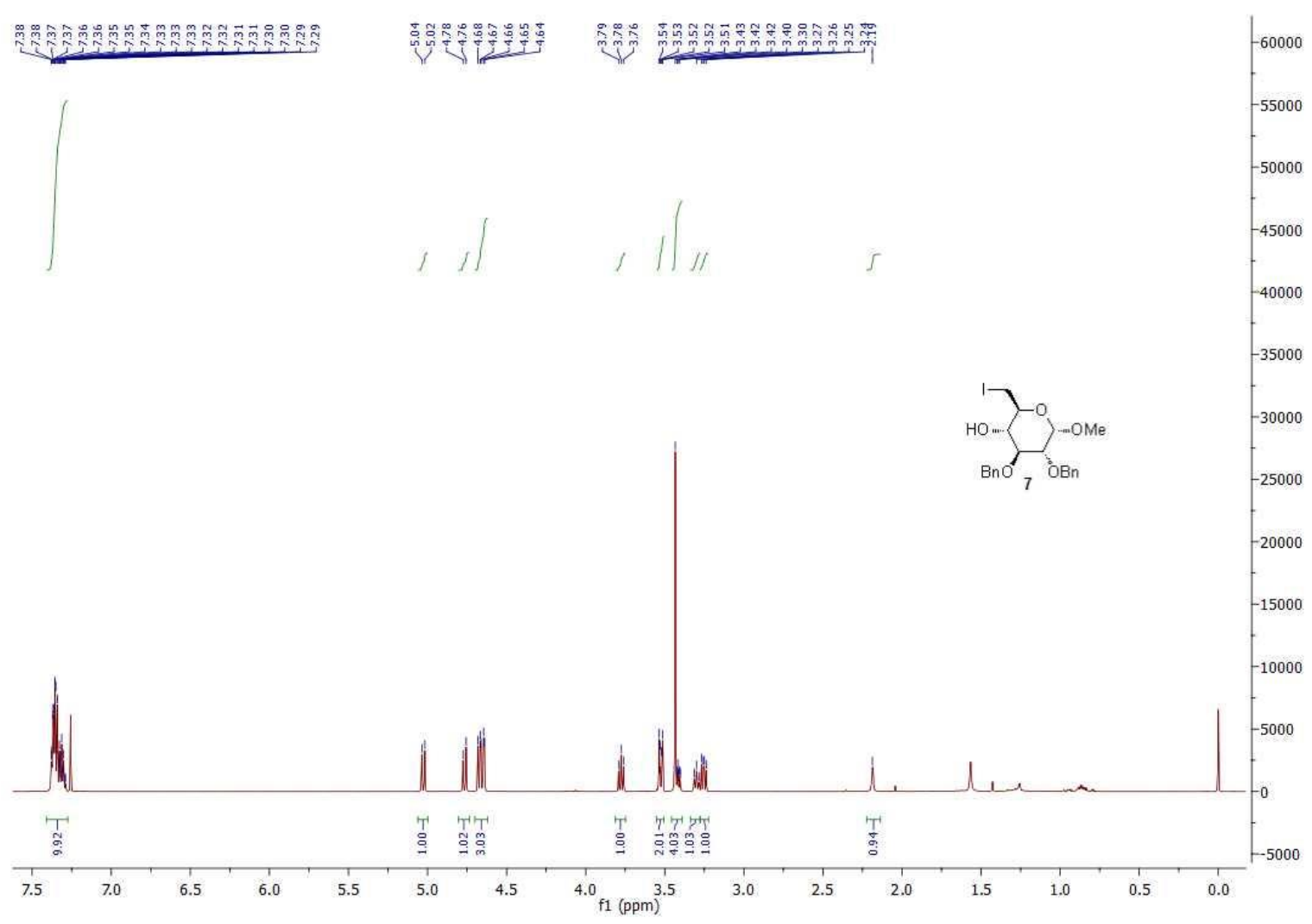

${ }^{1} \mathrm{H}-\mathrm{NMR}$ Spectrum of Compound $7\left(\mathrm{CDCl}_{3}\right)$

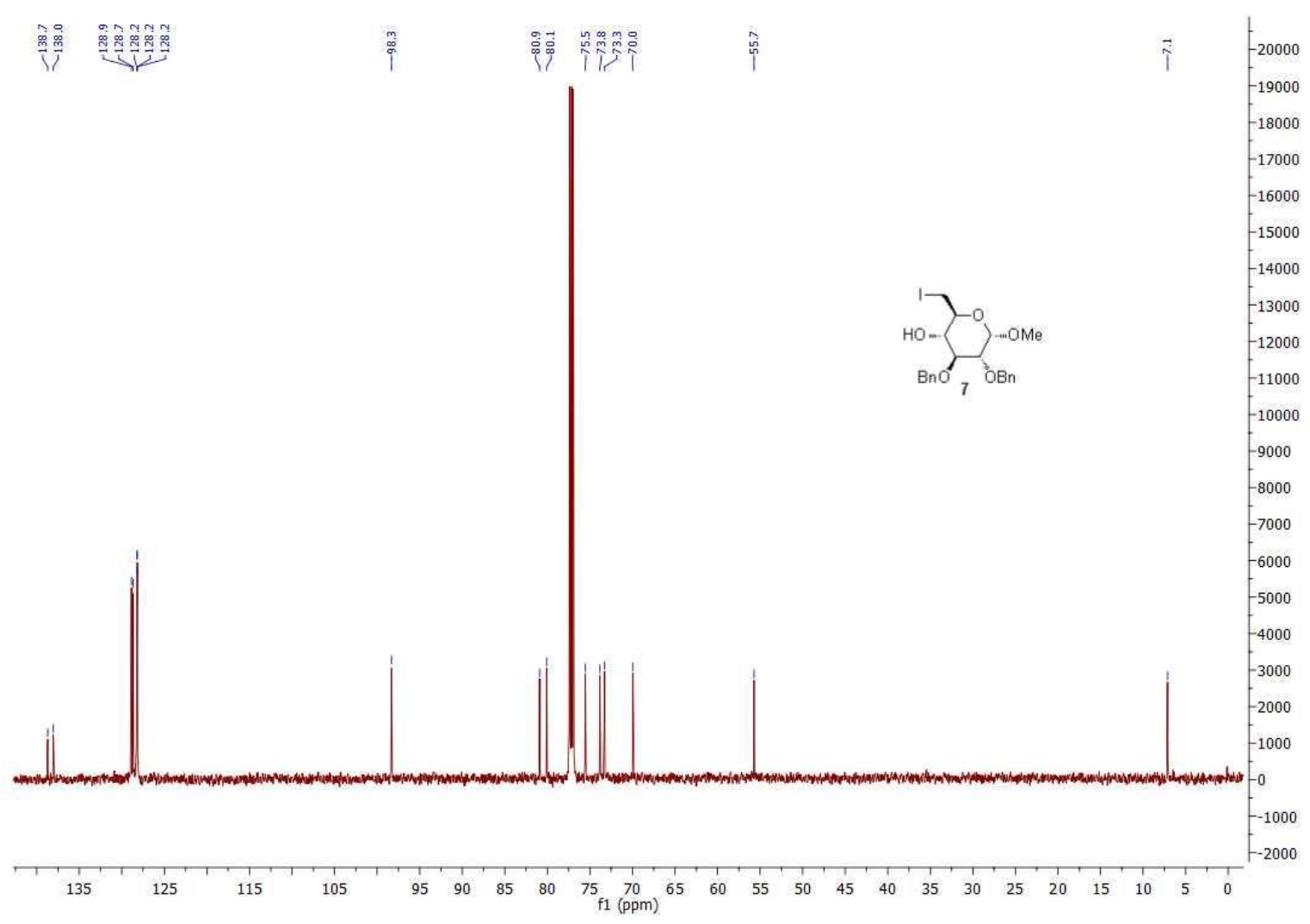

${ }^{13} \mathrm{C}$-NMR Spectrum of Compound $7\left(\mathrm{CDCl}_{3}\right)$ 


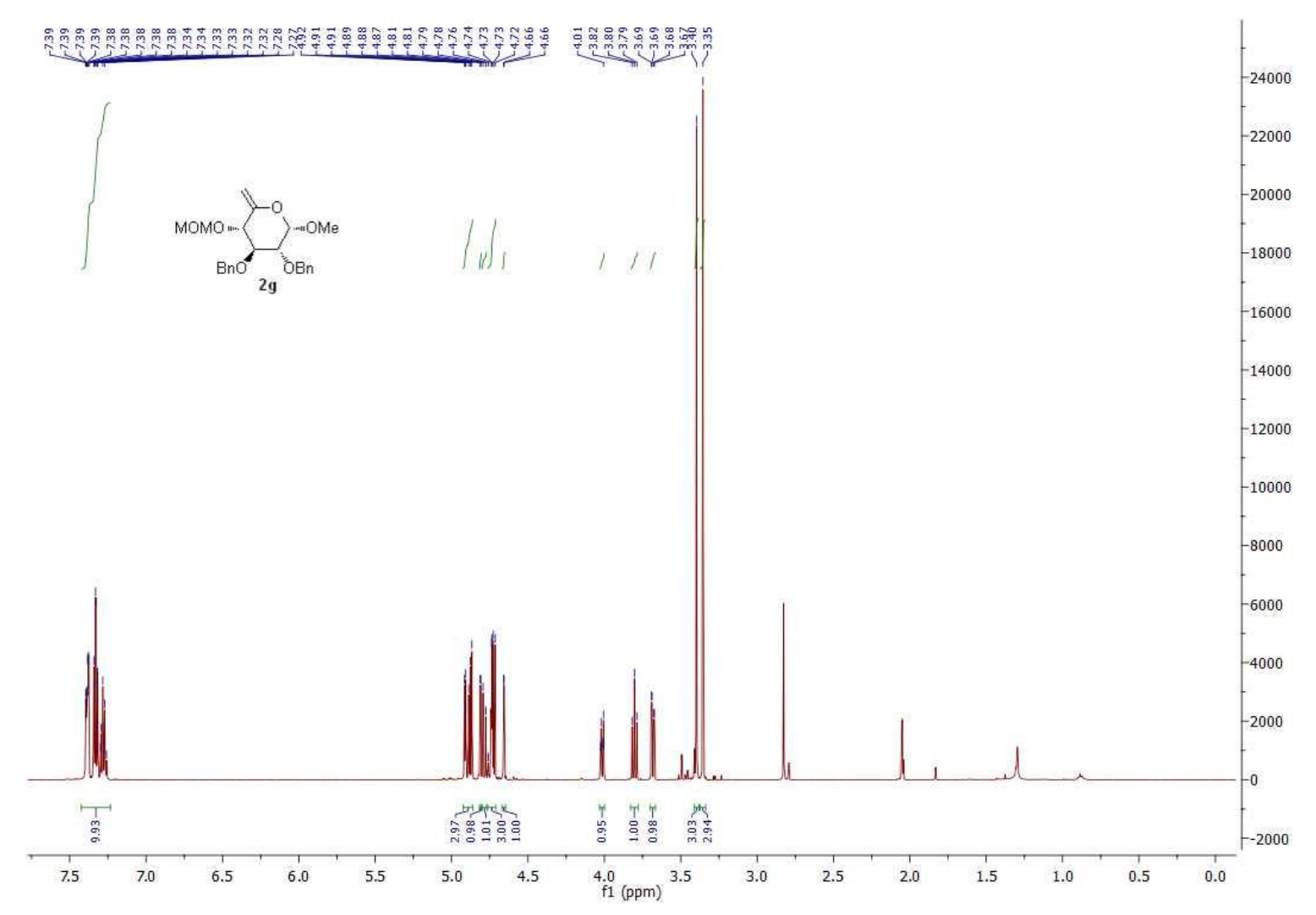

${ }^{1} \mathrm{H}-\mathrm{NMR}$ Spectrum of Compound 2g (Acetone-d6)

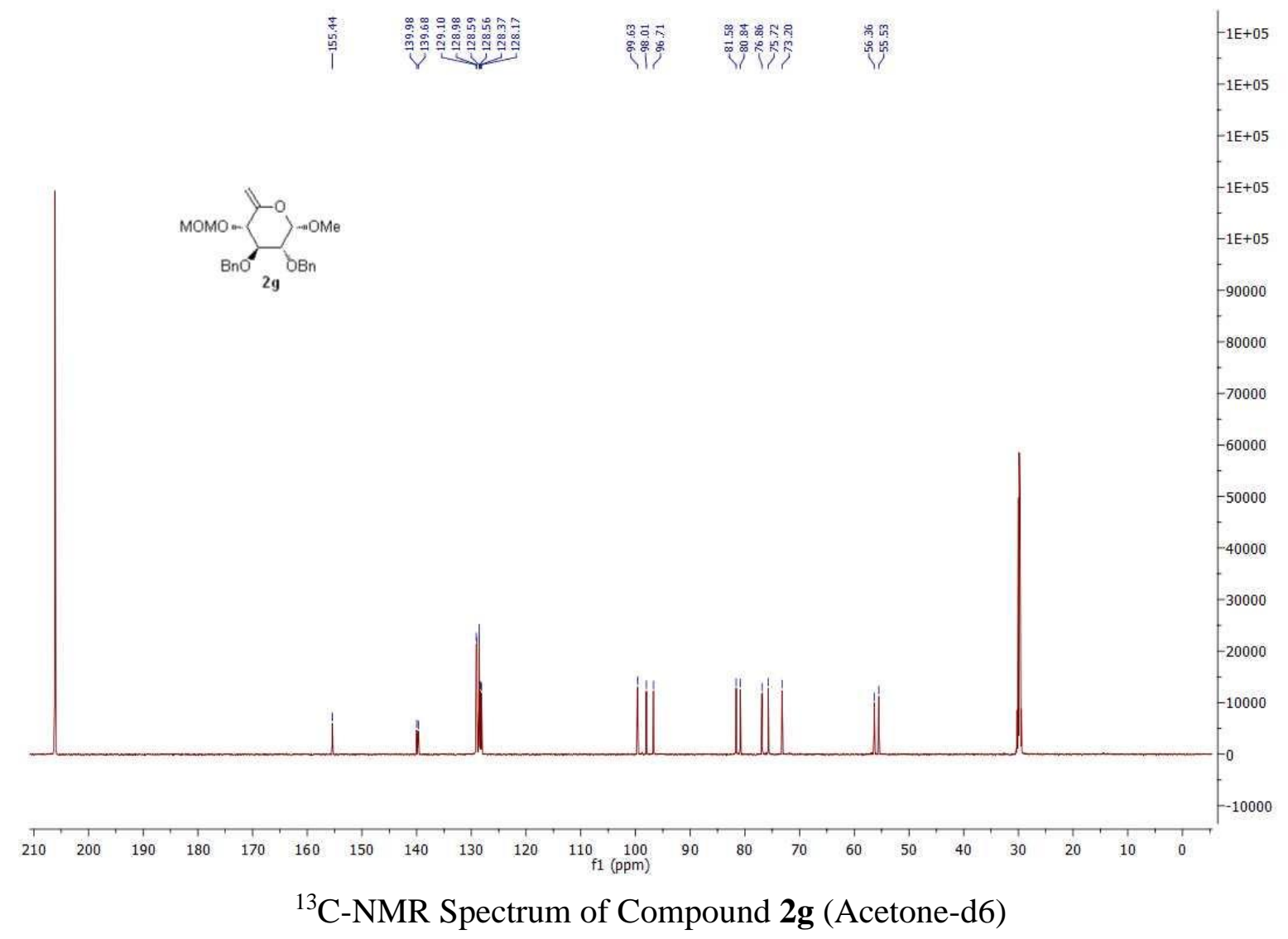




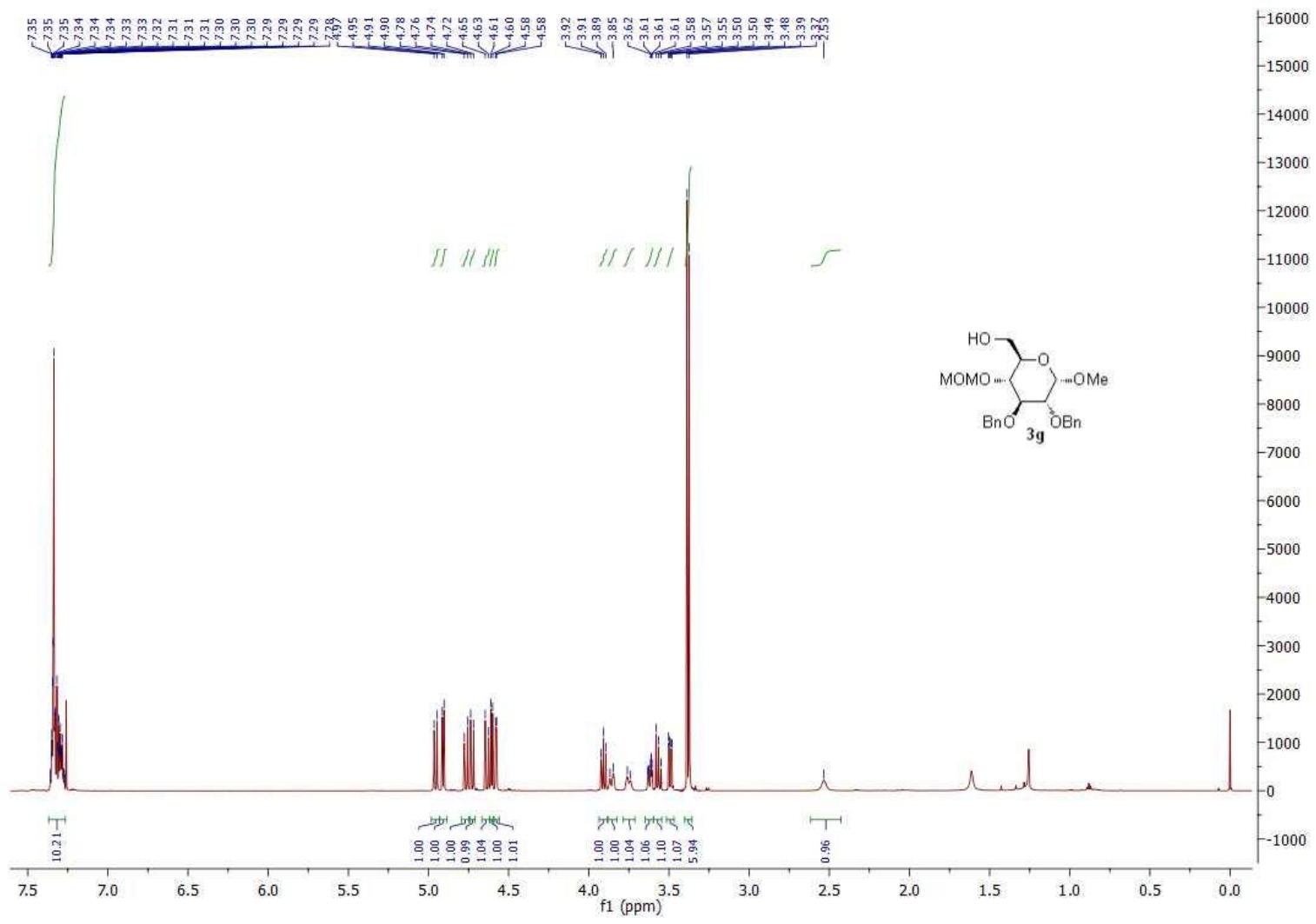

${ }^{1} \mathrm{H}-\mathrm{NMR}$ Spectrum of Compound $\mathbf{3 g}\left(\mathrm{CDCl}_{3}\right)$

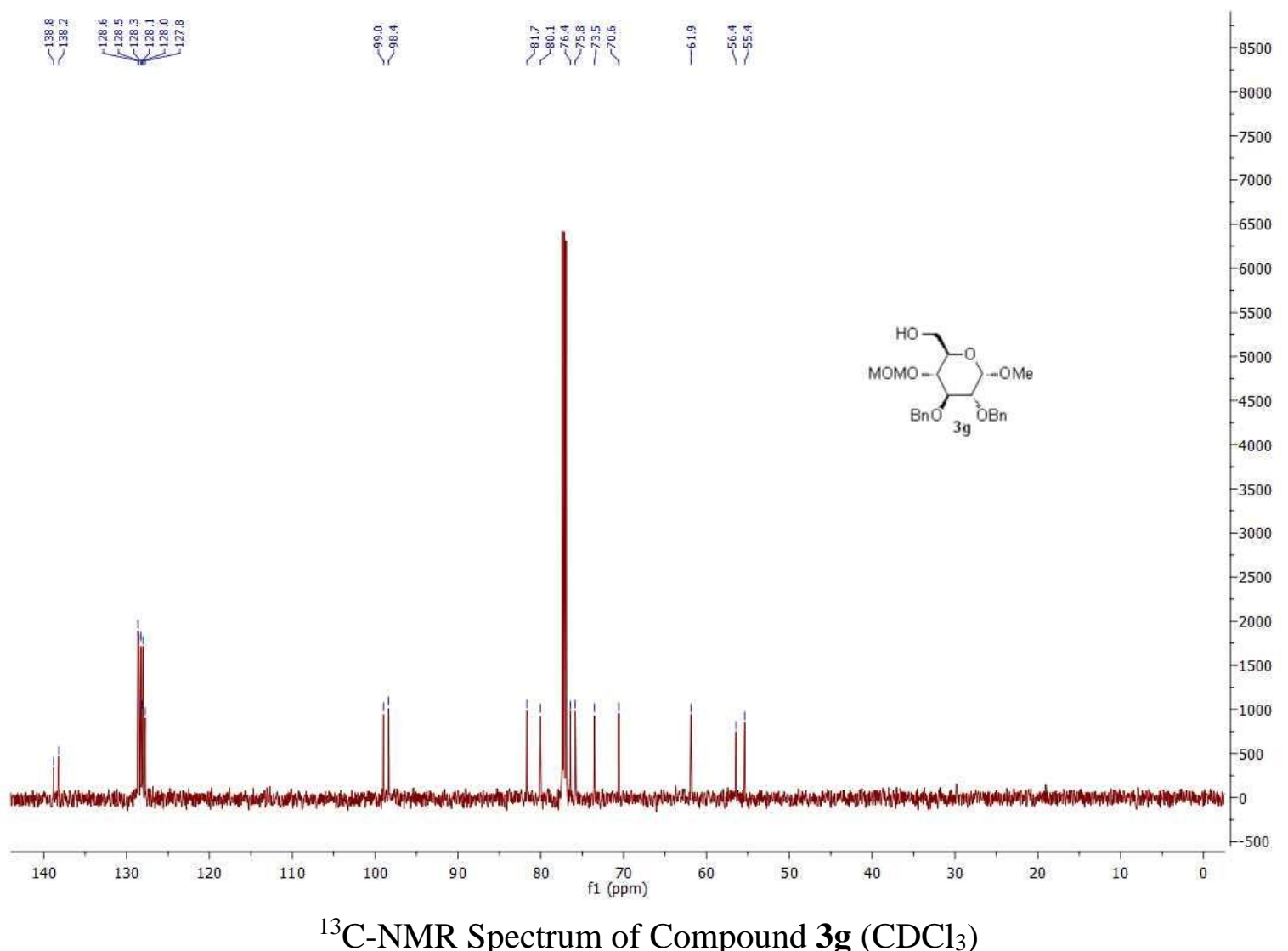




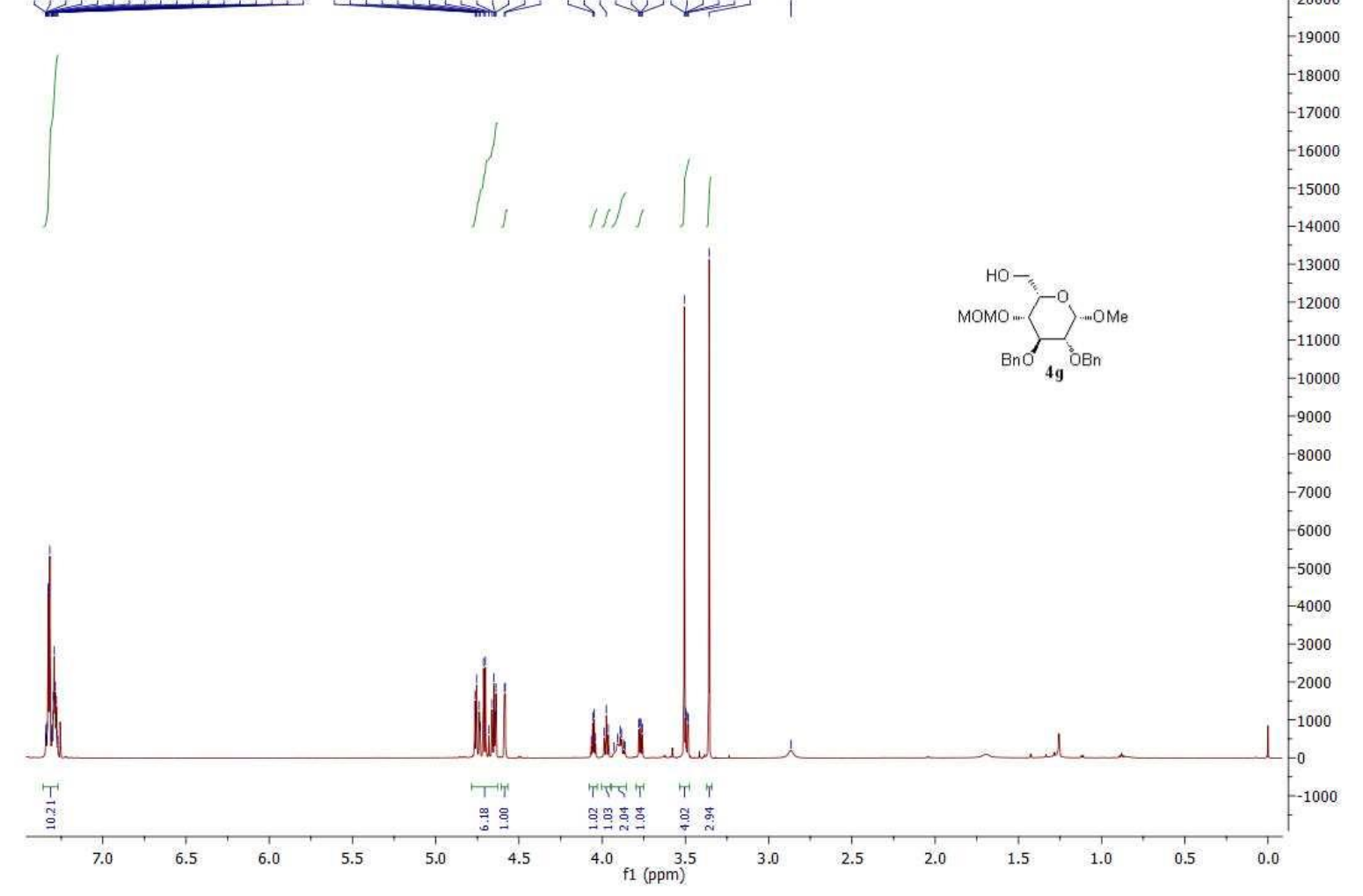

${ }^{1} \mathrm{H}-\mathrm{NMR}$ Spectrum of Compound $\mathbf{4 g}\left(\mathrm{CDCl}_{3}\right)$

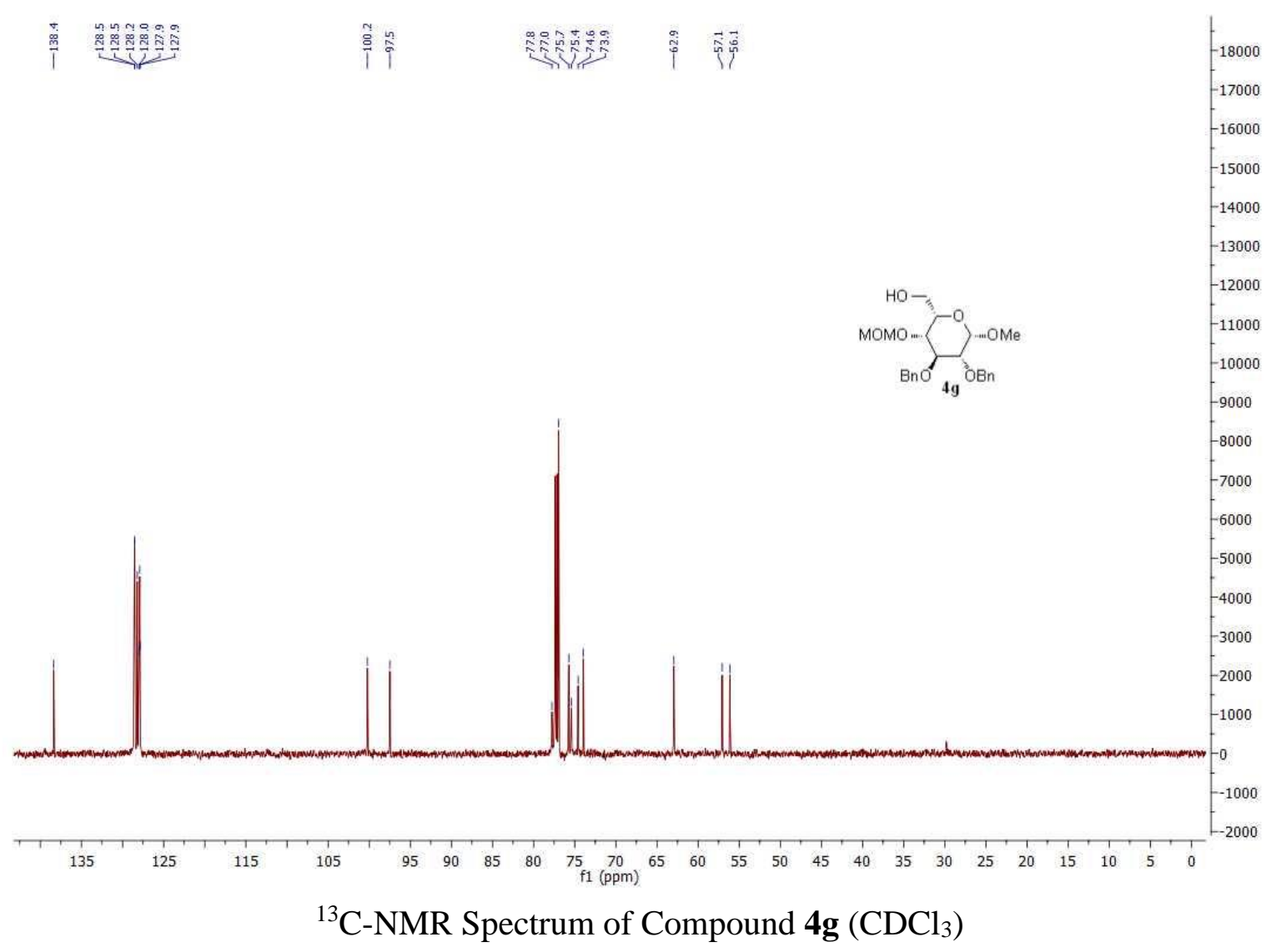




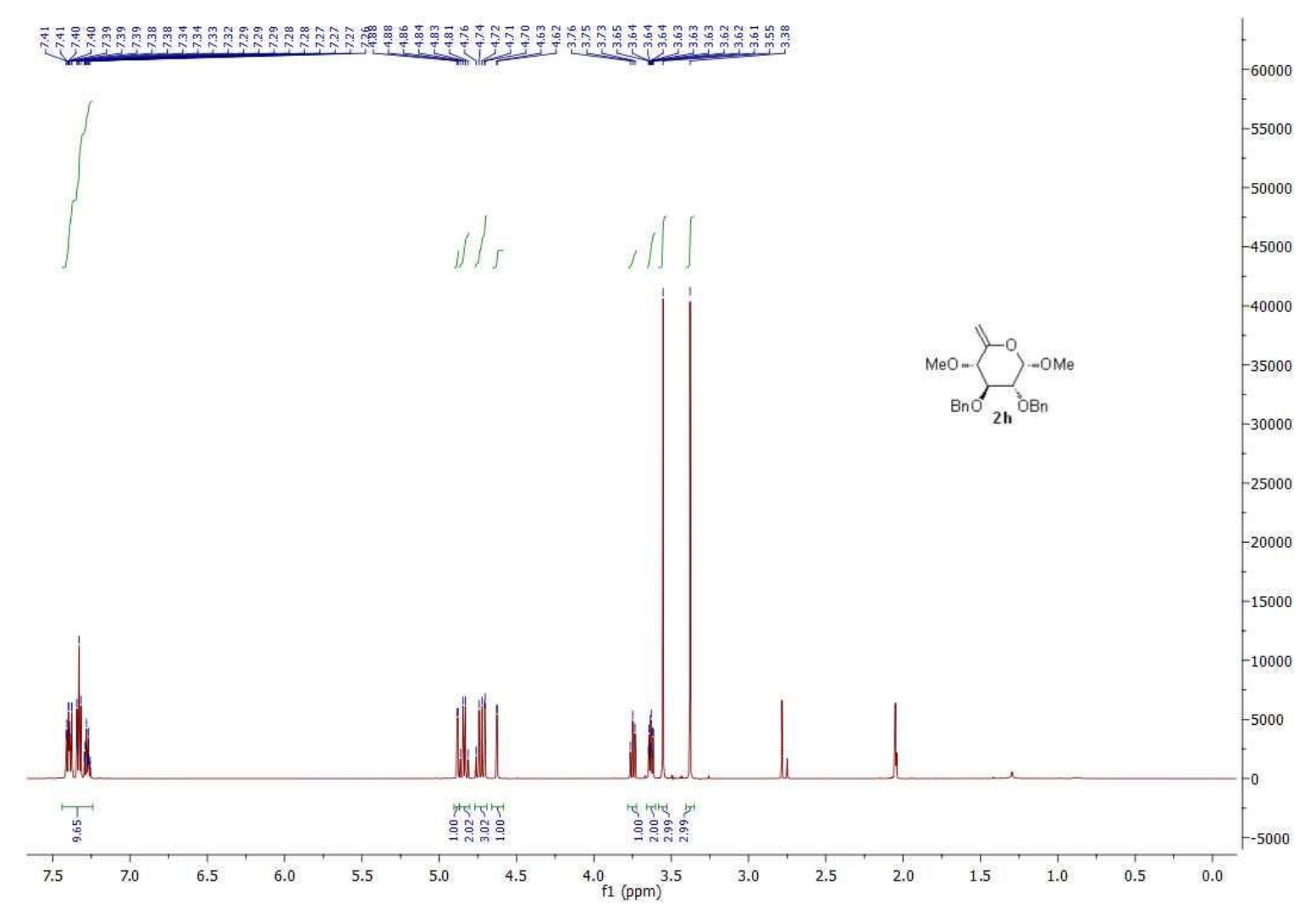

${ }^{1}$ H-NMR Spectrum of Compound $\mathbf{2 h}$ (Acetone-d6)

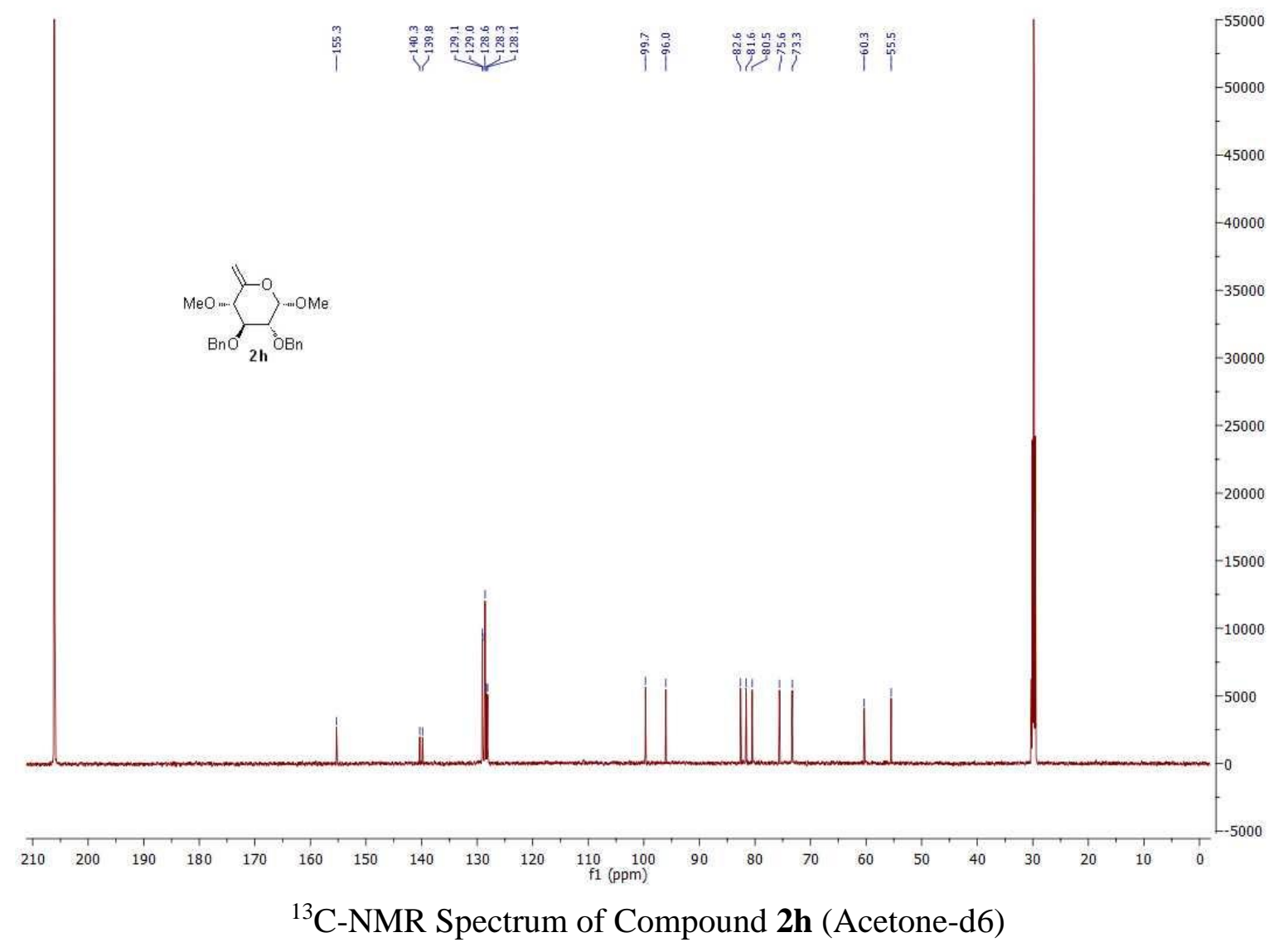




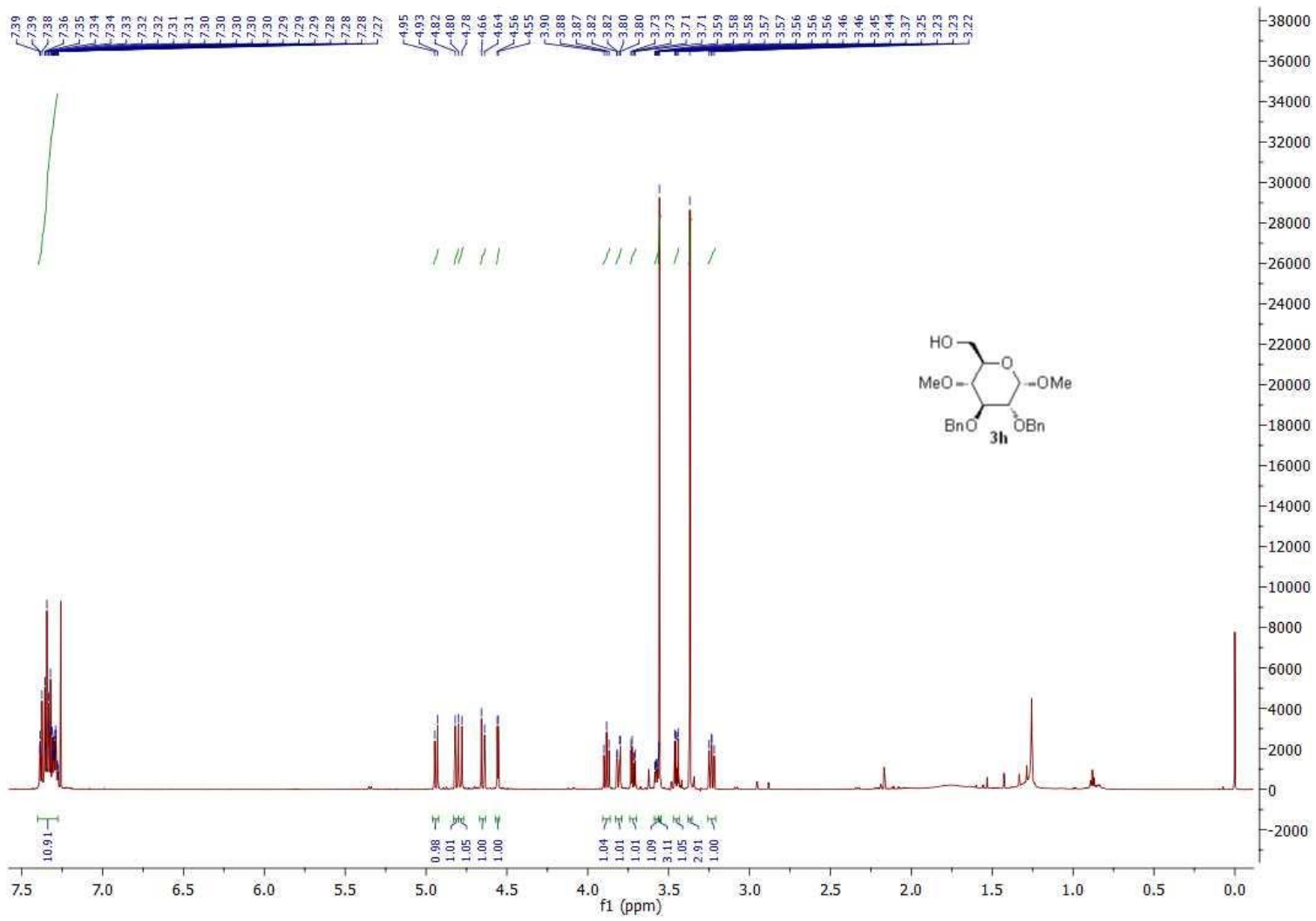

${ }^{1} \mathrm{H}-\mathrm{NMR}$ Spectrum of Compound $\mathbf{3 h}\left(\mathrm{CDCl}_{3}\right)$

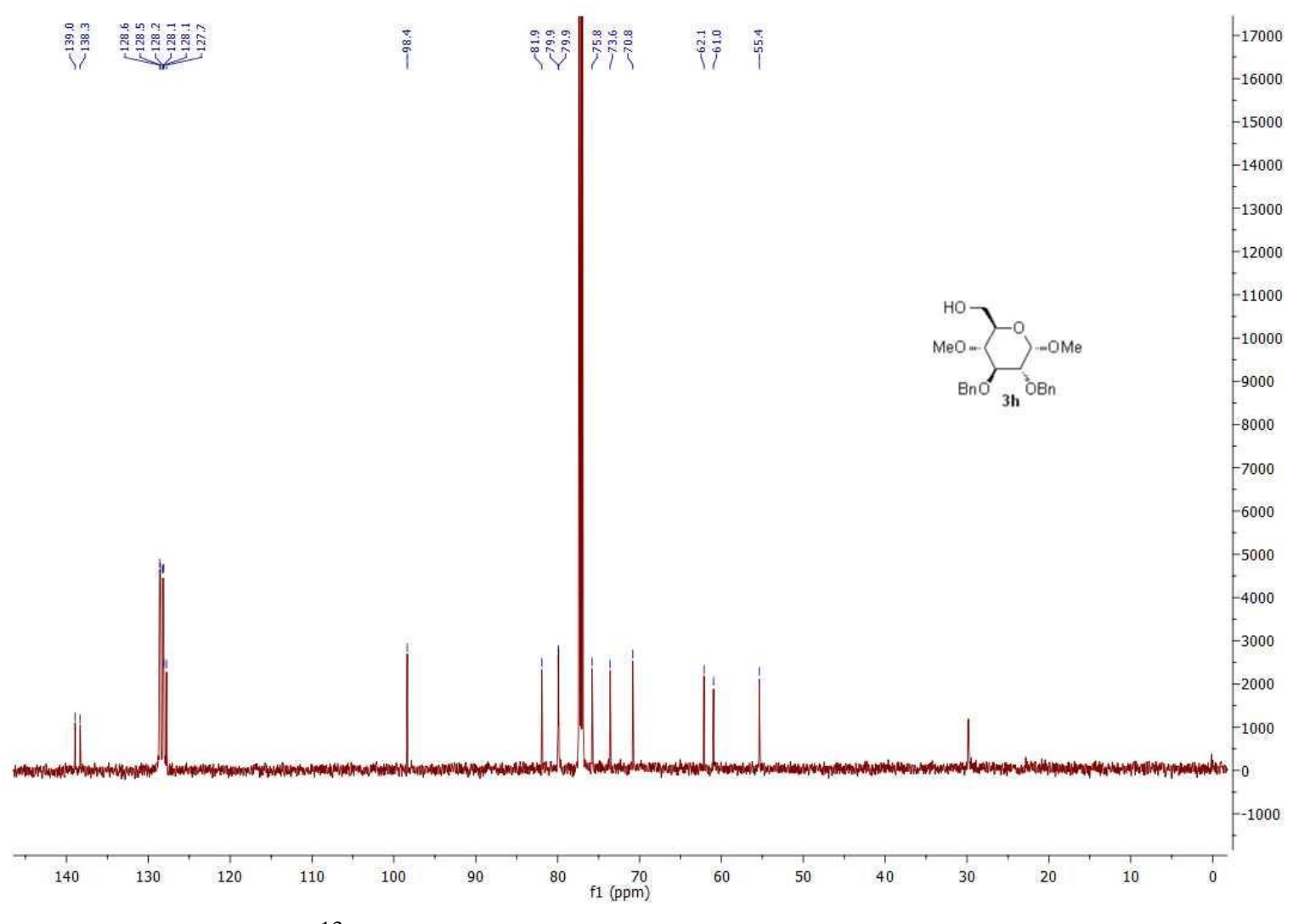

${ }^{13} \mathrm{C}-\mathrm{NMR}$ Spectrum of Compound $\mathbf{3 h}\left(\mathrm{CDCl}_{3}\right)$ 


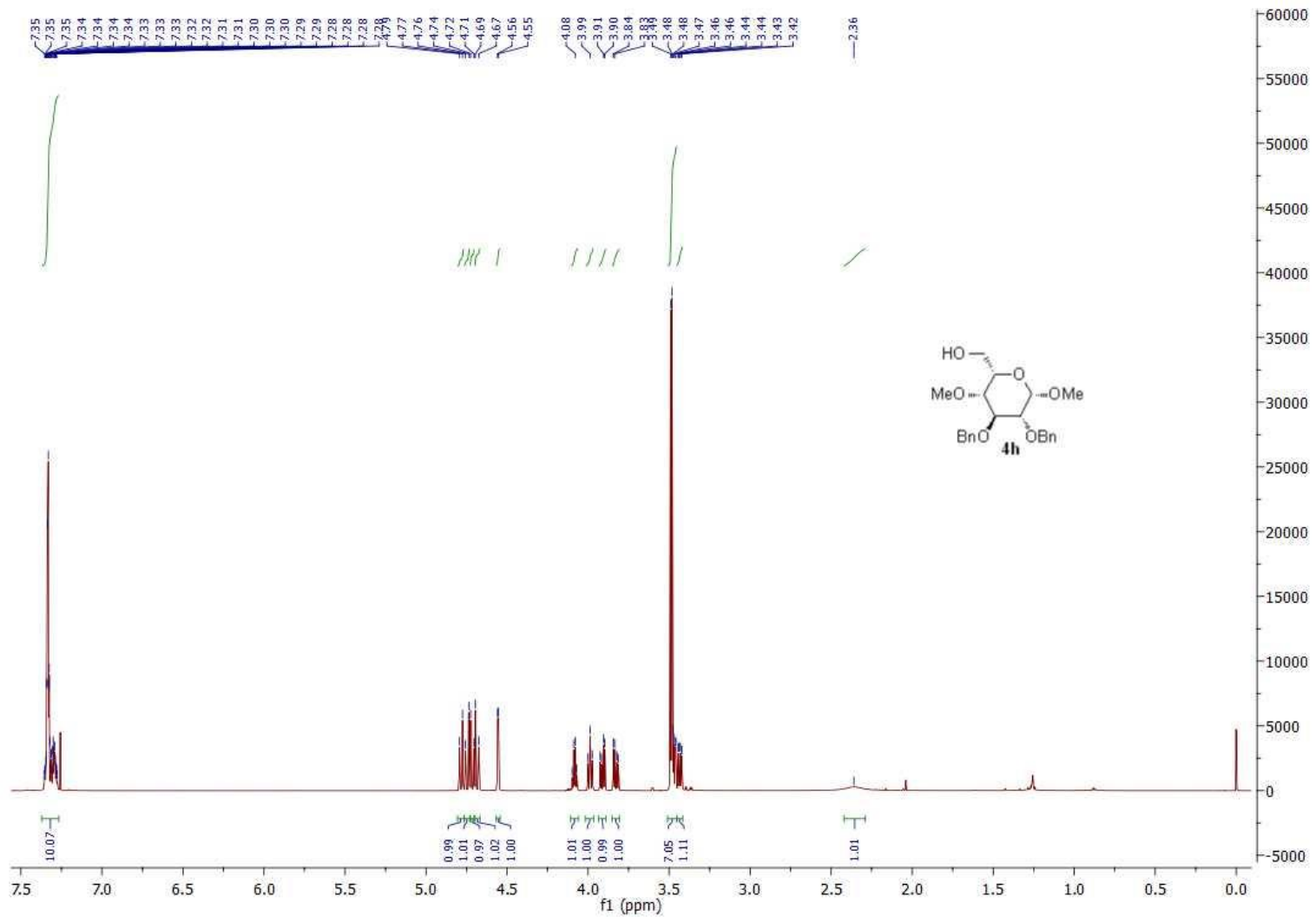

${ }^{1} \mathrm{H}-\mathrm{NMR}$ Spectrum of Compound $\mathbf{4 h}\left(\mathrm{CDCl}_{3}\right)$

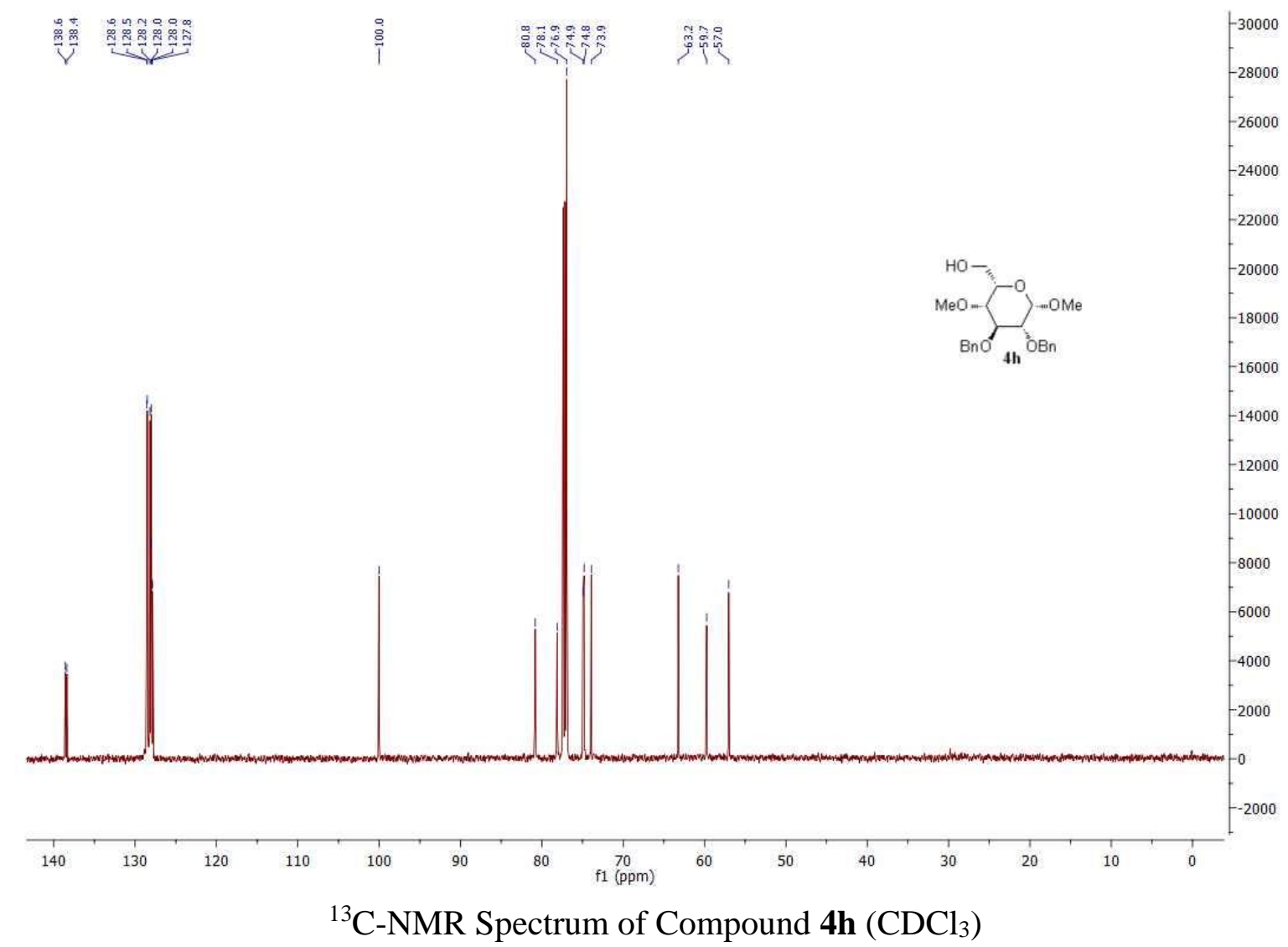




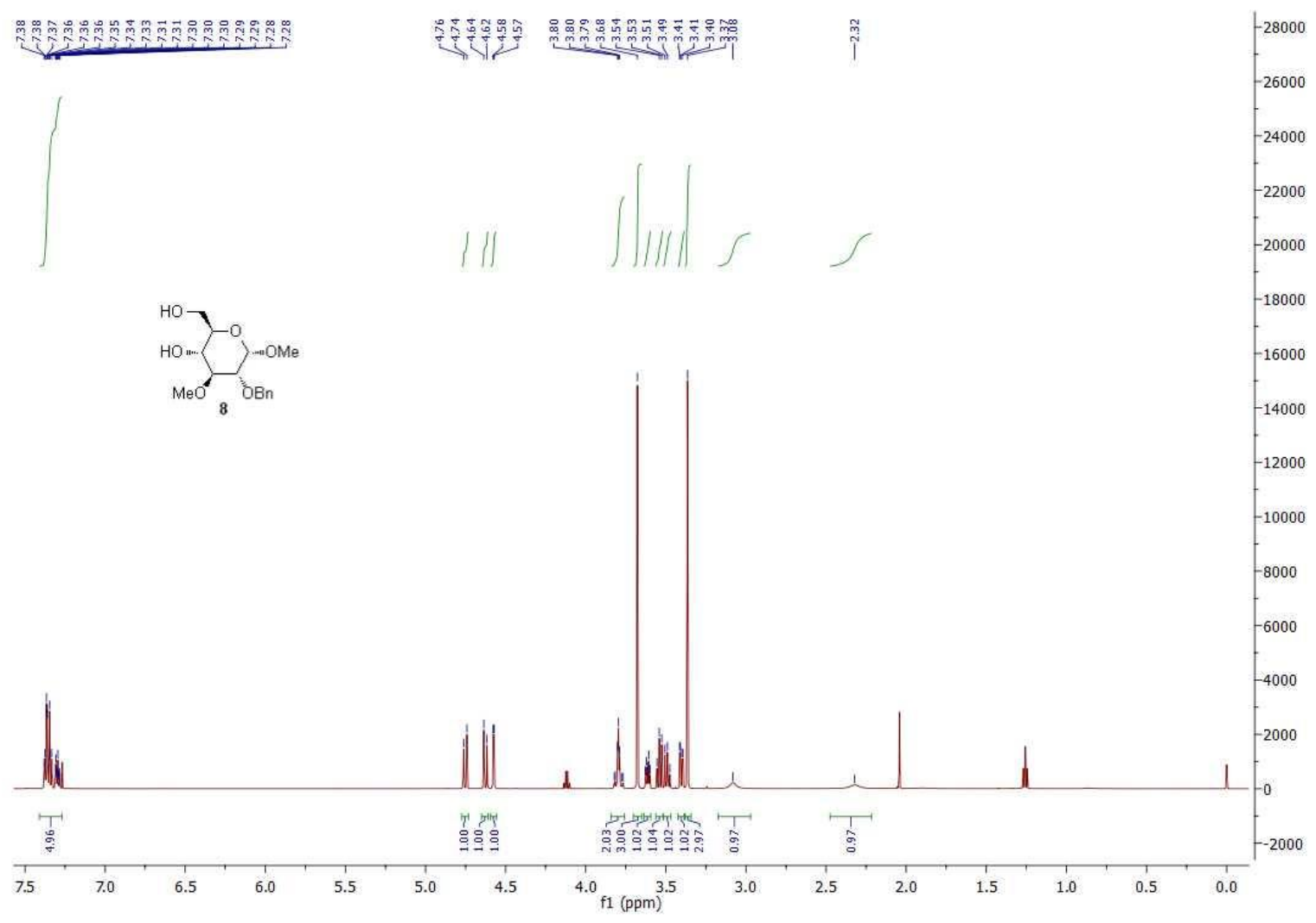

${ }^{1} \mathrm{H}-\mathrm{NMR}$ Spectrum of Compound $\mathbf{8}\left(\mathrm{CDCl}_{3}\right)$

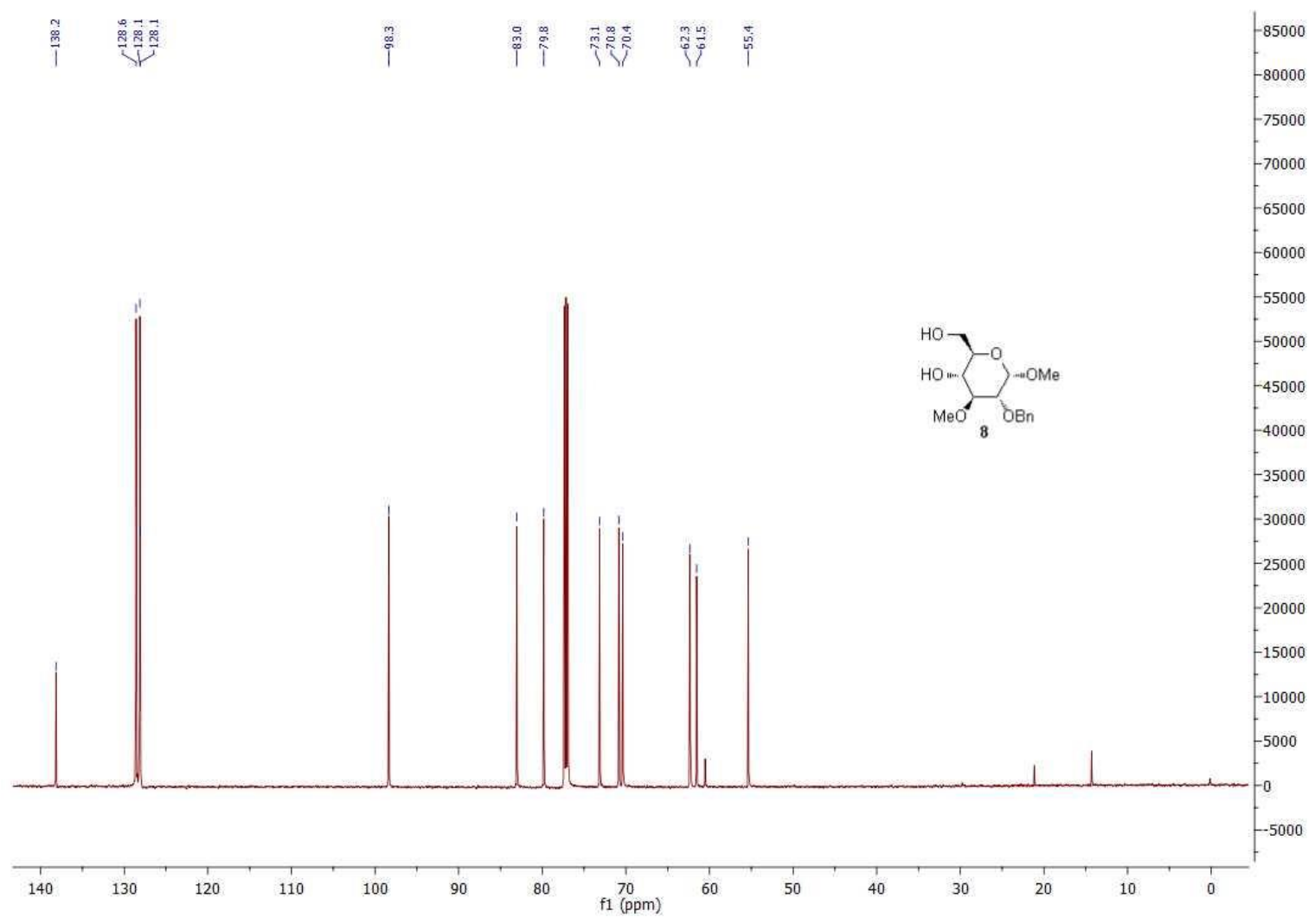

${ }^{13} \mathrm{C}$-NMR Spectrum of Compound $\mathbf{8}\left(\mathrm{CDCl}_{3}\right)$ 


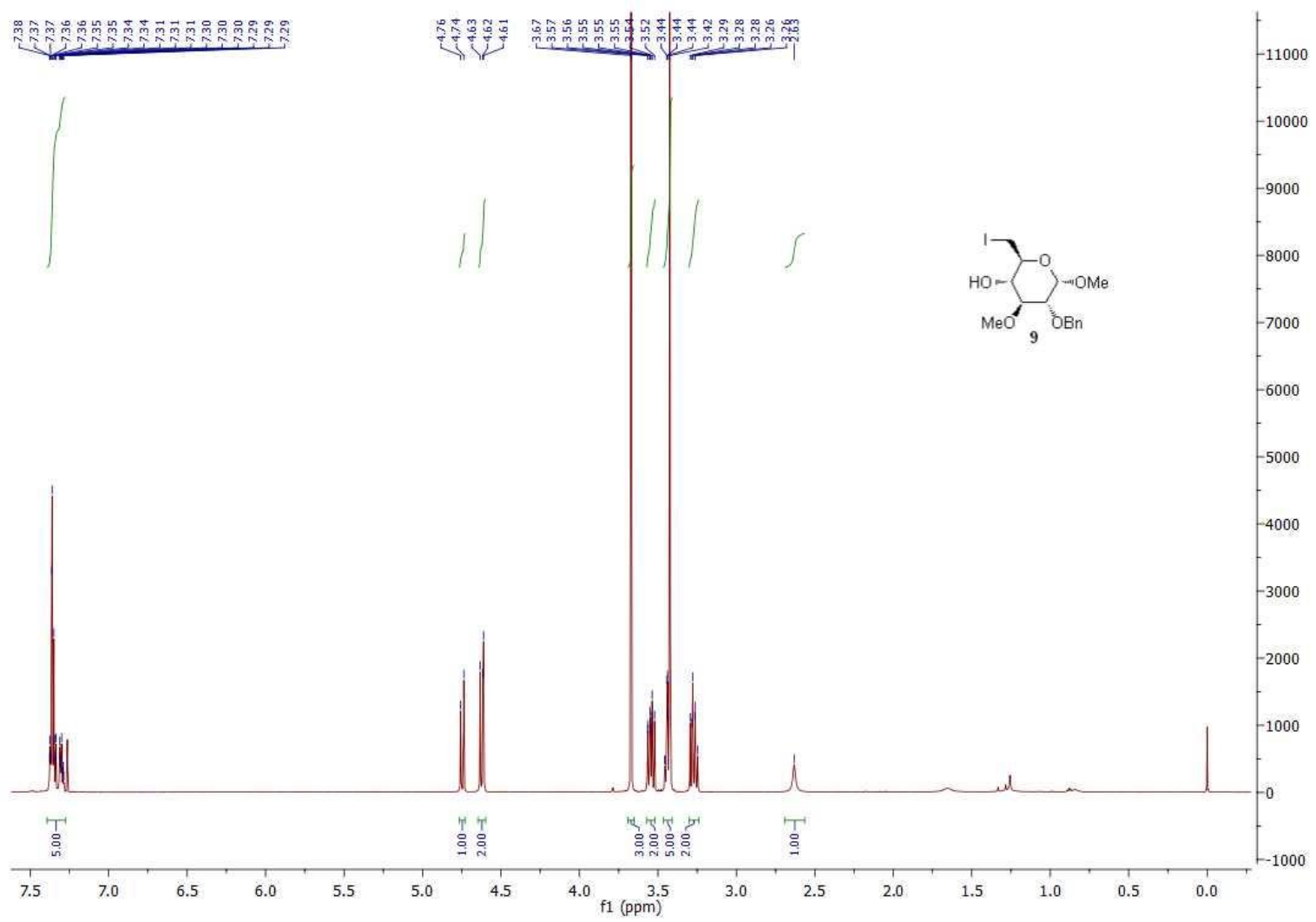

${ }^{1} \mathrm{H}-\mathrm{NMR}$ Spectrum of Compound $9\left(\mathrm{CDCl}_{3}\right)$

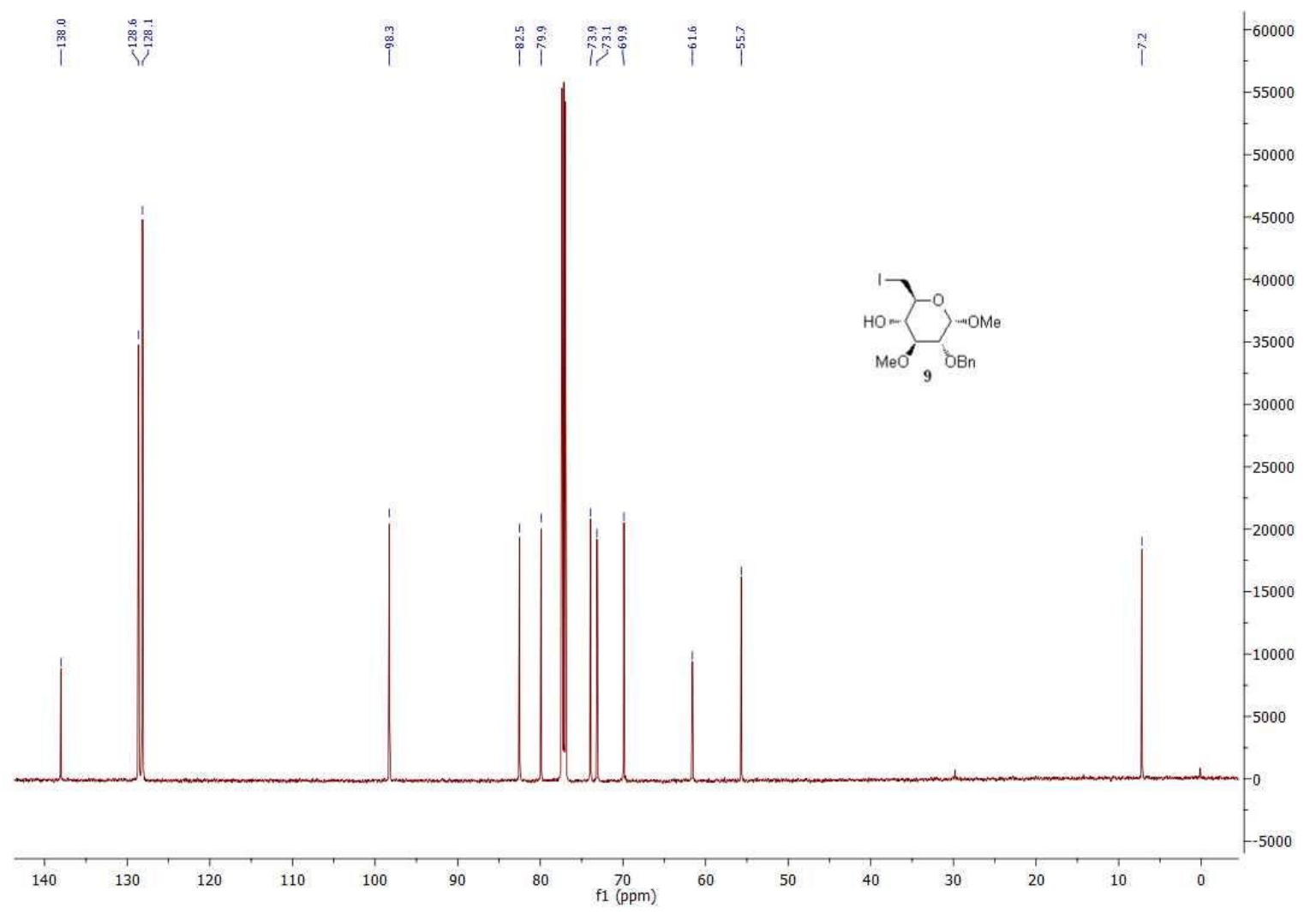

${ }^{13} \mathrm{C}$-NMR Spectrum of Compound $9\left(\mathrm{CDCl}_{3}\right)$ 


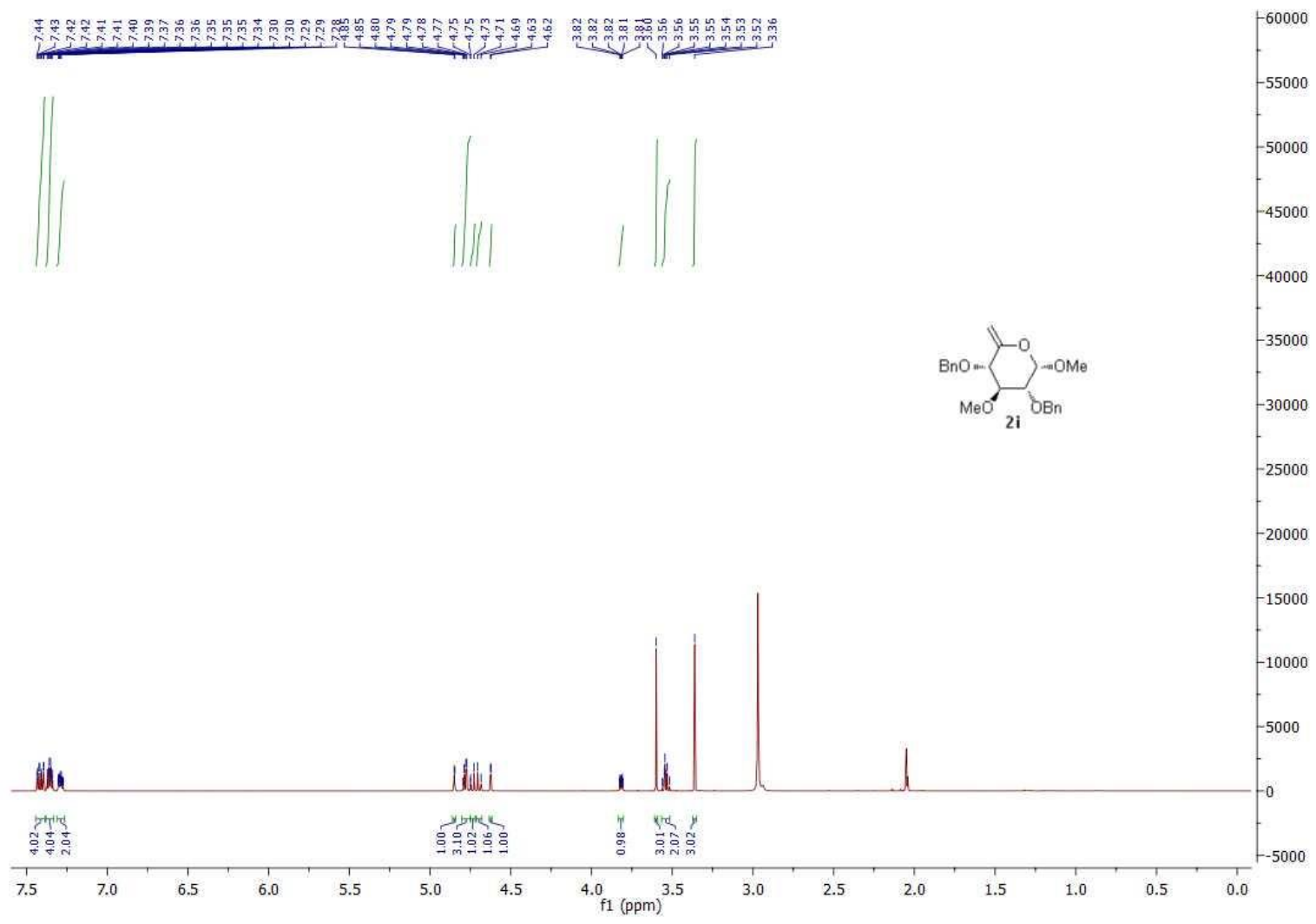

${ }^{1}$ H-NMR Spectrum of Compound 2i (Acetone-d6)

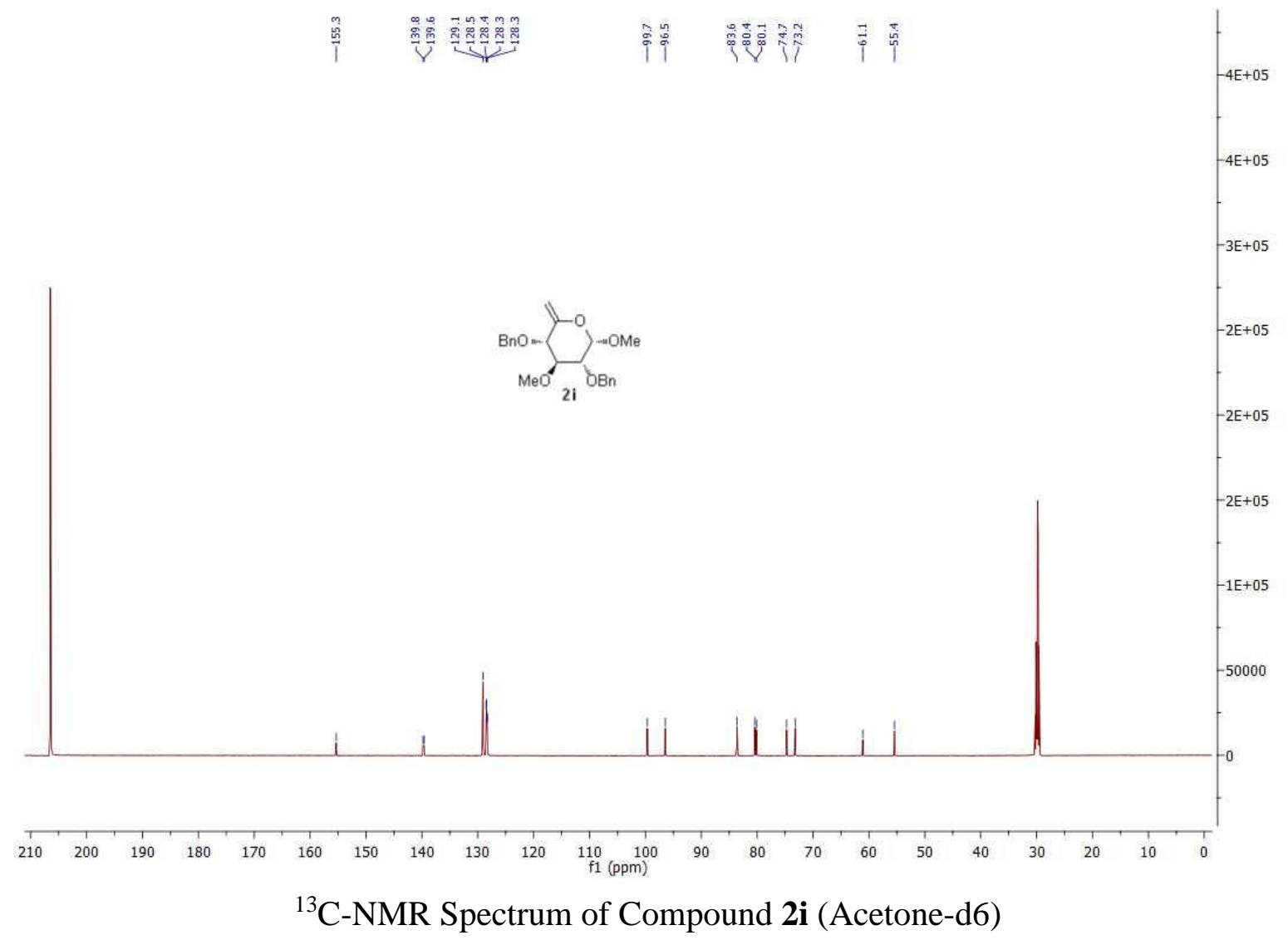




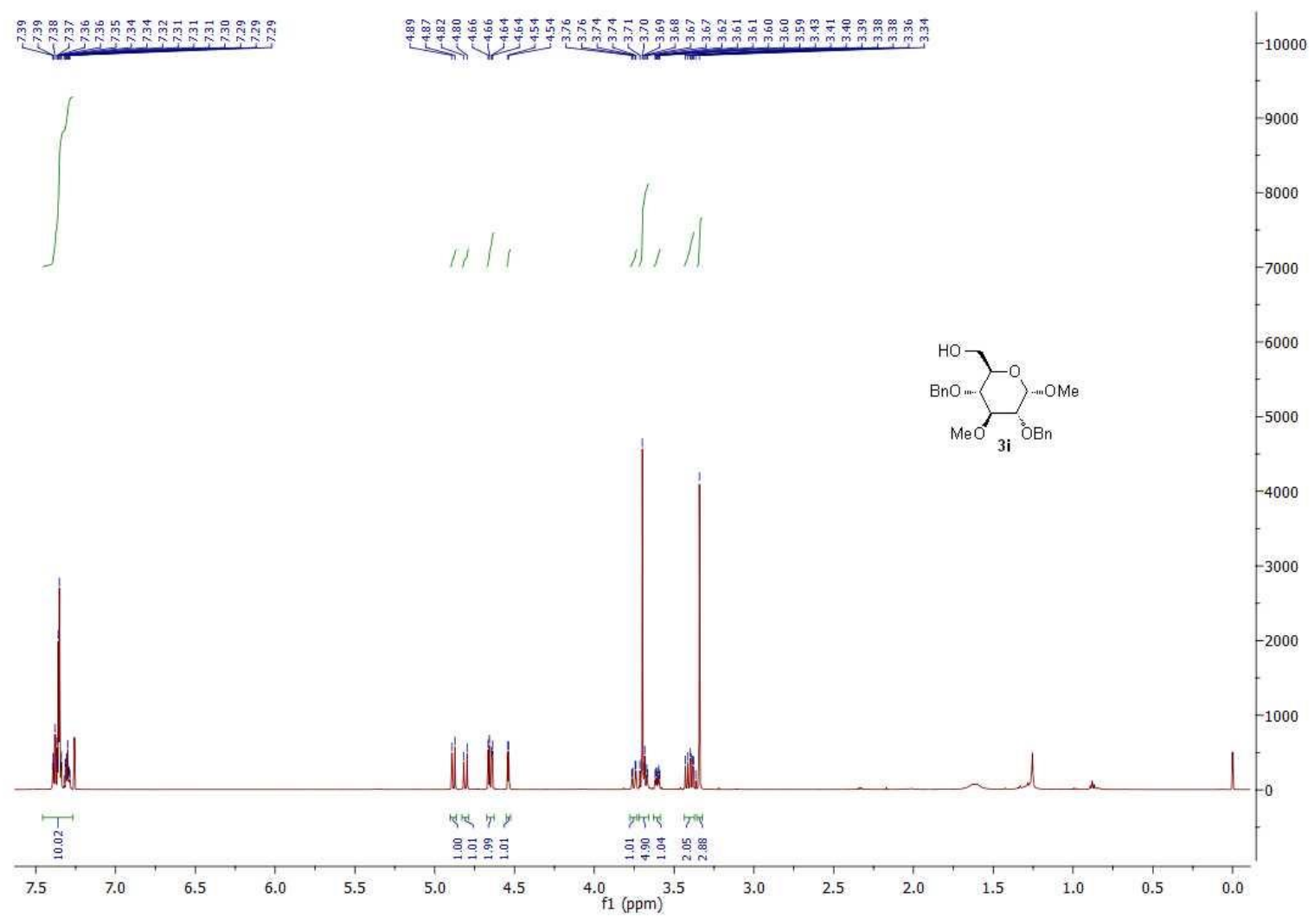

${ }^{1} \mathrm{H}-\mathrm{NMR}$ Spectrum of Compound $\mathbf{3 i}\left(\mathrm{CDCl}_{3}\right)$

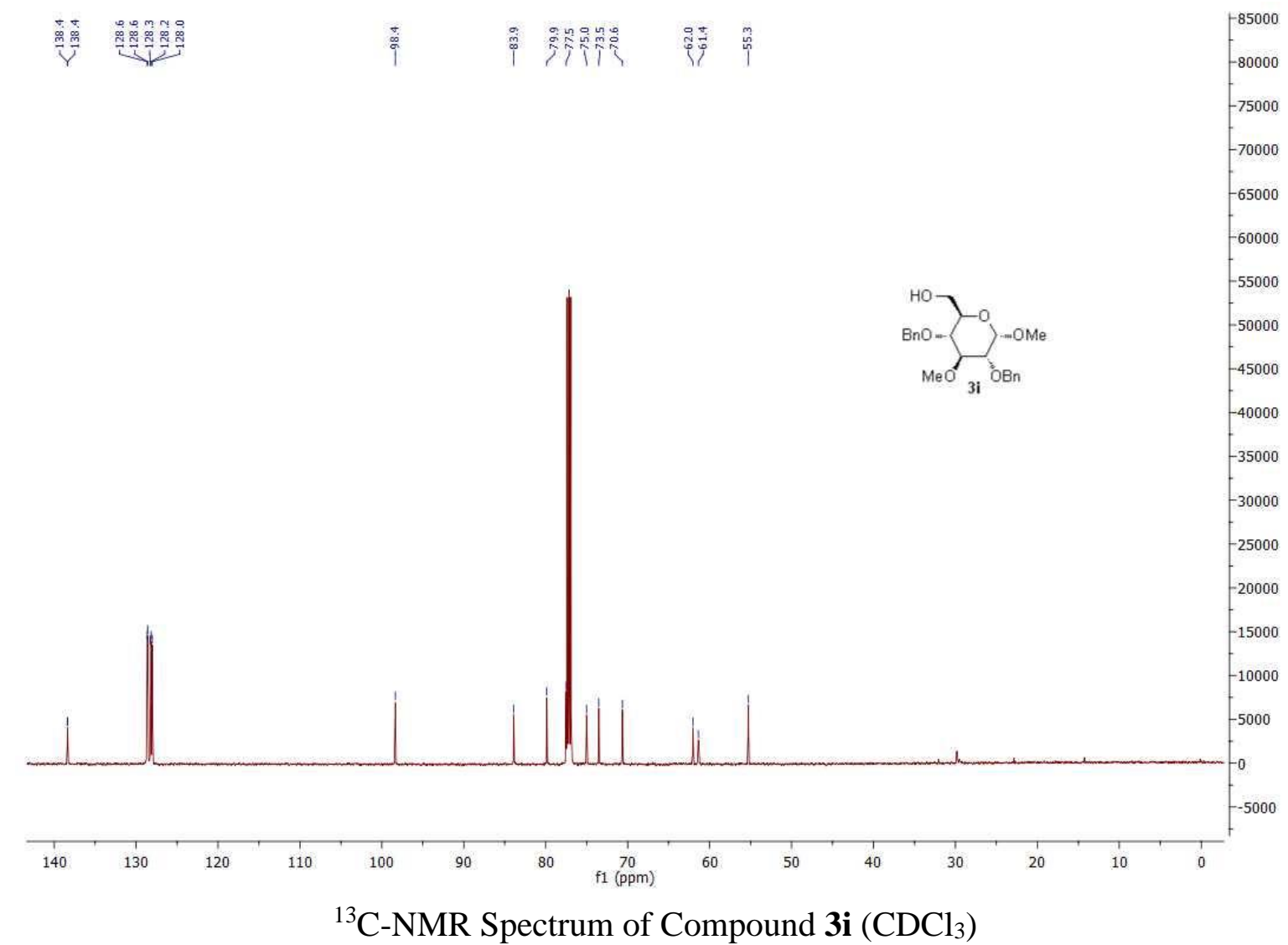




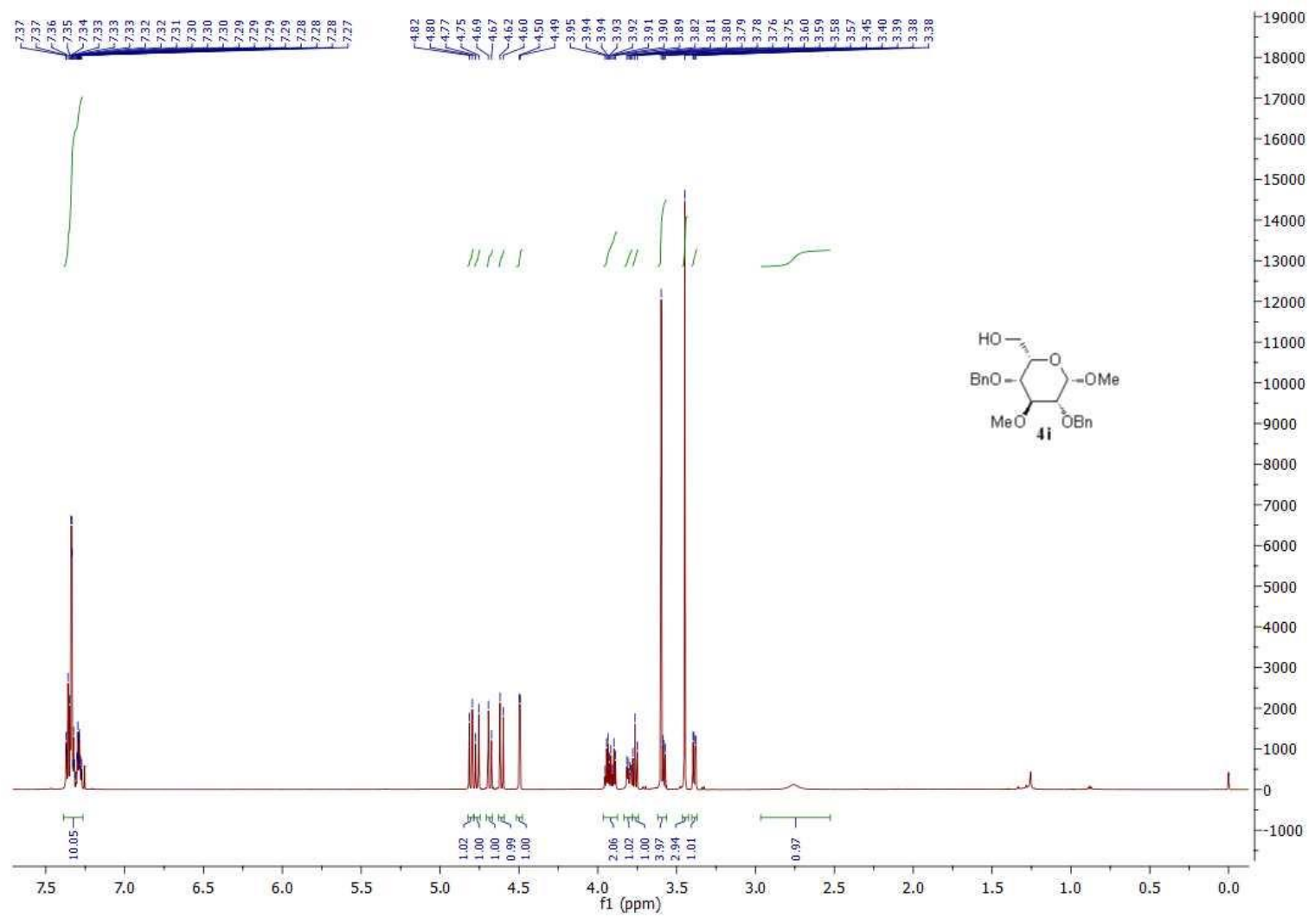

${ }^{1} \mathrm{H}-\mathrm{NMR}$ Spectrum of Compound $\mathbf{4 i}\left(\mathrm{CDCl}_{3}\right)$

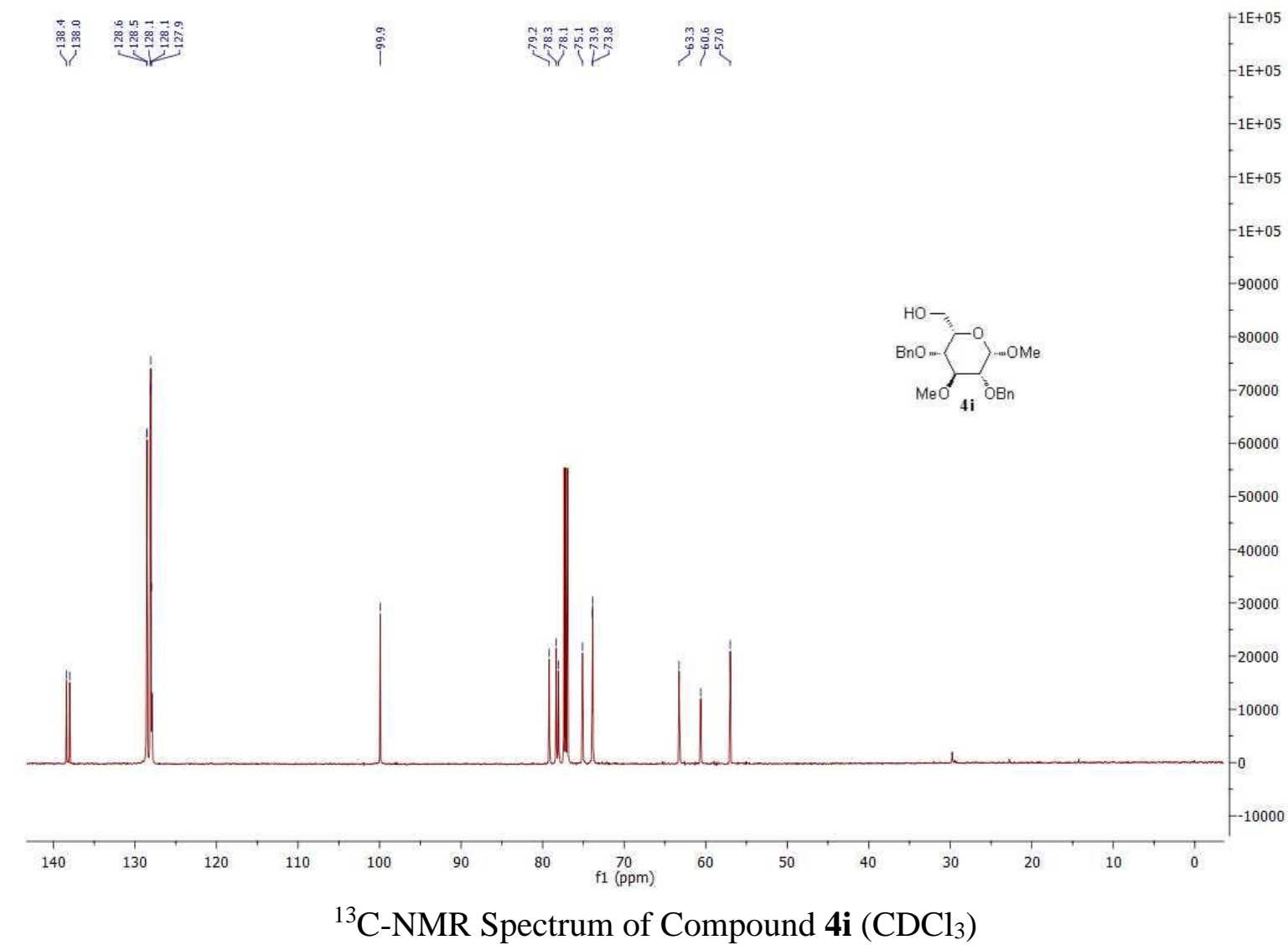




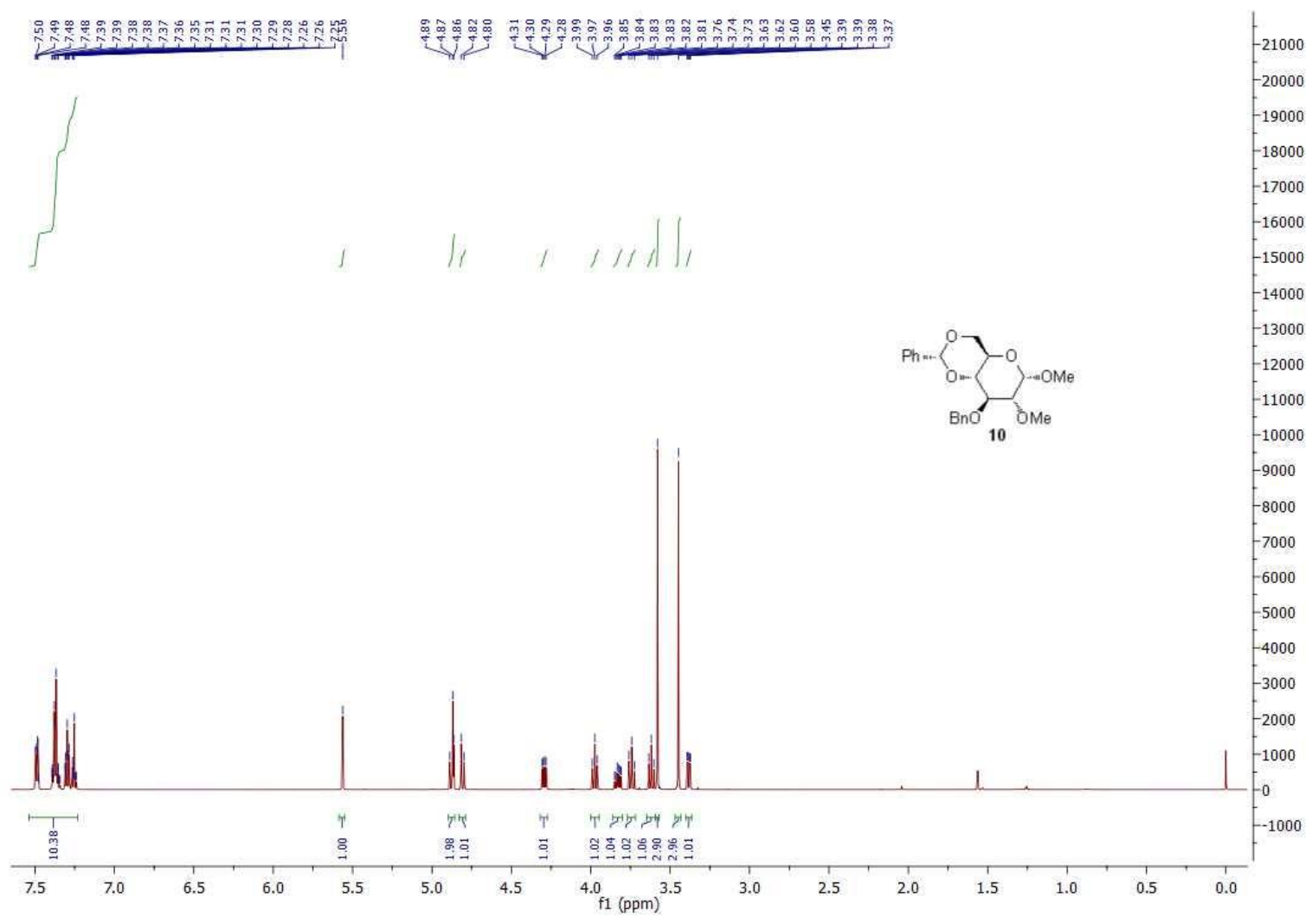

${ }^{1} \mathrm{H}-\mathrm{NMR}$ Spectrum of Compound $\mathbf{1 0}\left(\mathrm{CDCl}_{3}\right)$

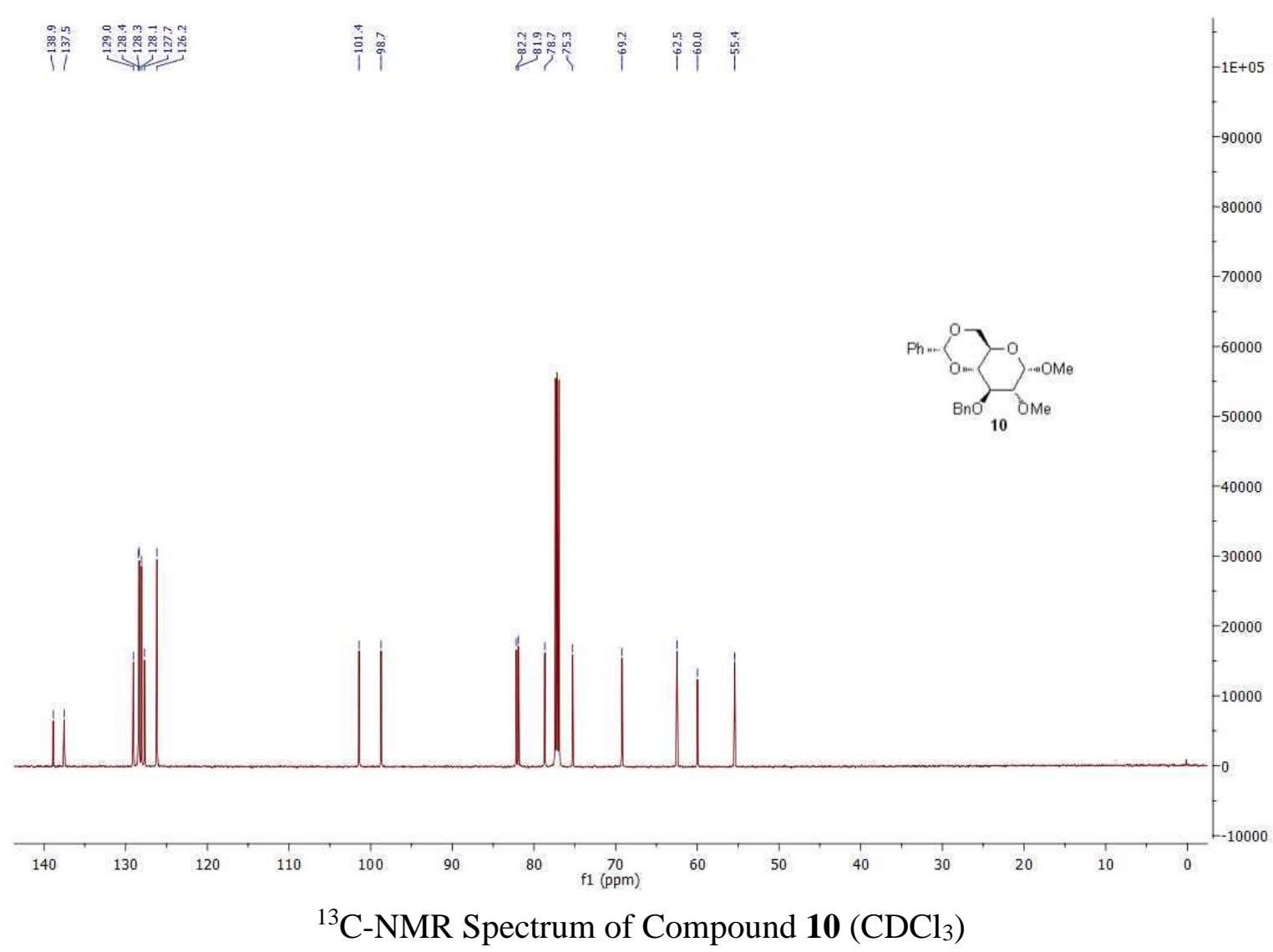




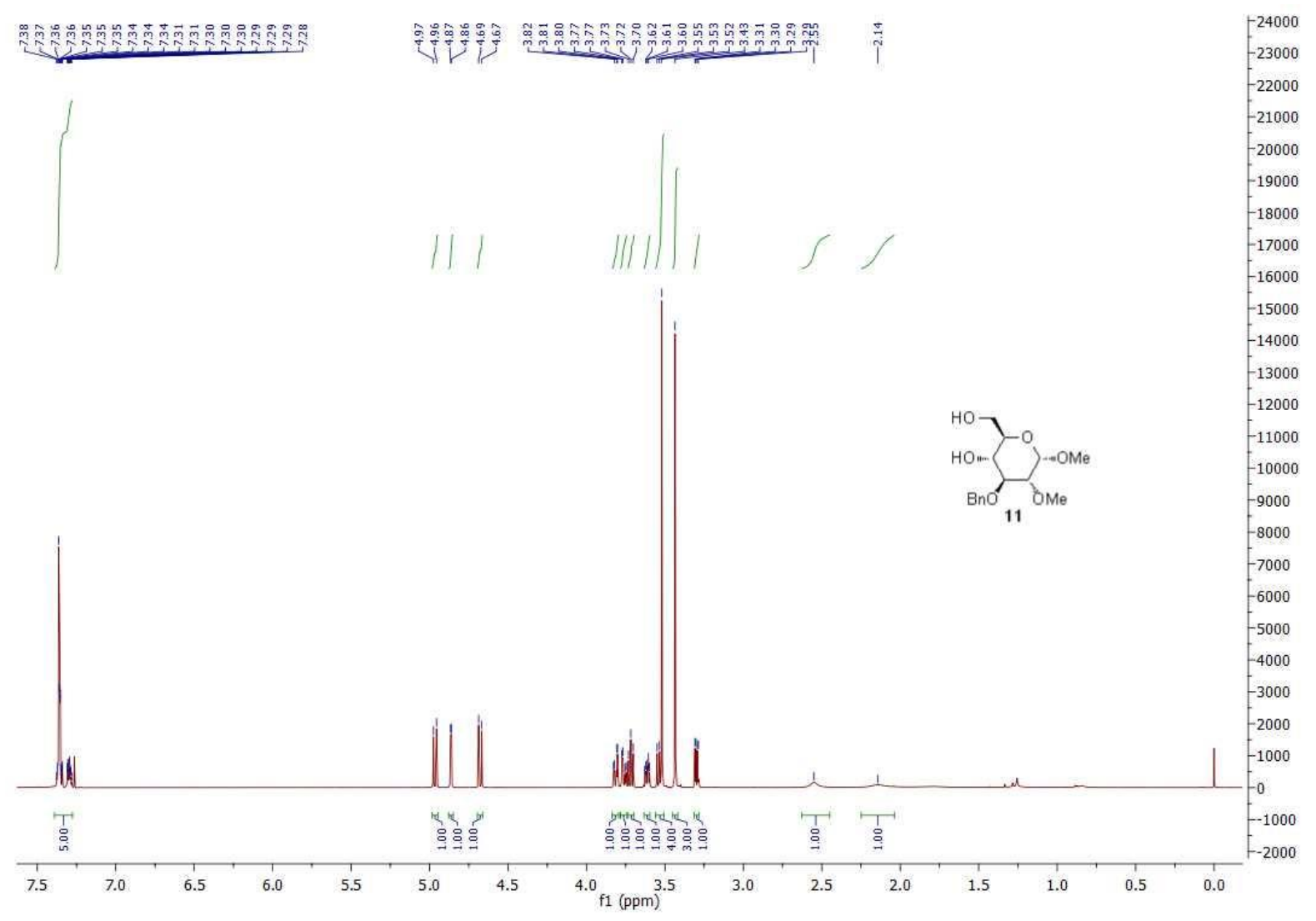

${ }^{1} \mathrm{H}-\mathrm{NMR}$ Spectrum of Compound $\mathbf{1 1}\left(\mathrm{CDCl}_{3}\right)$

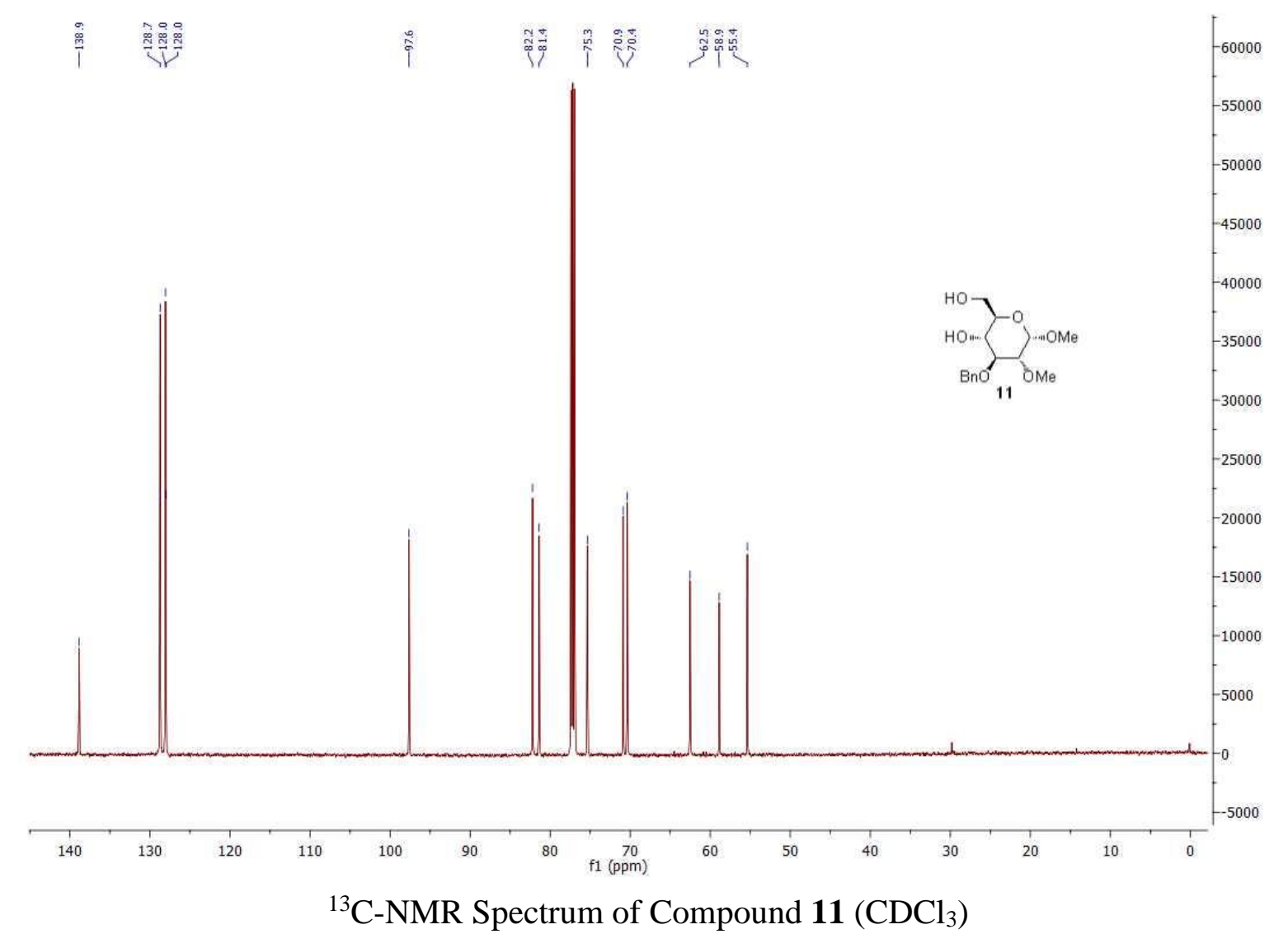




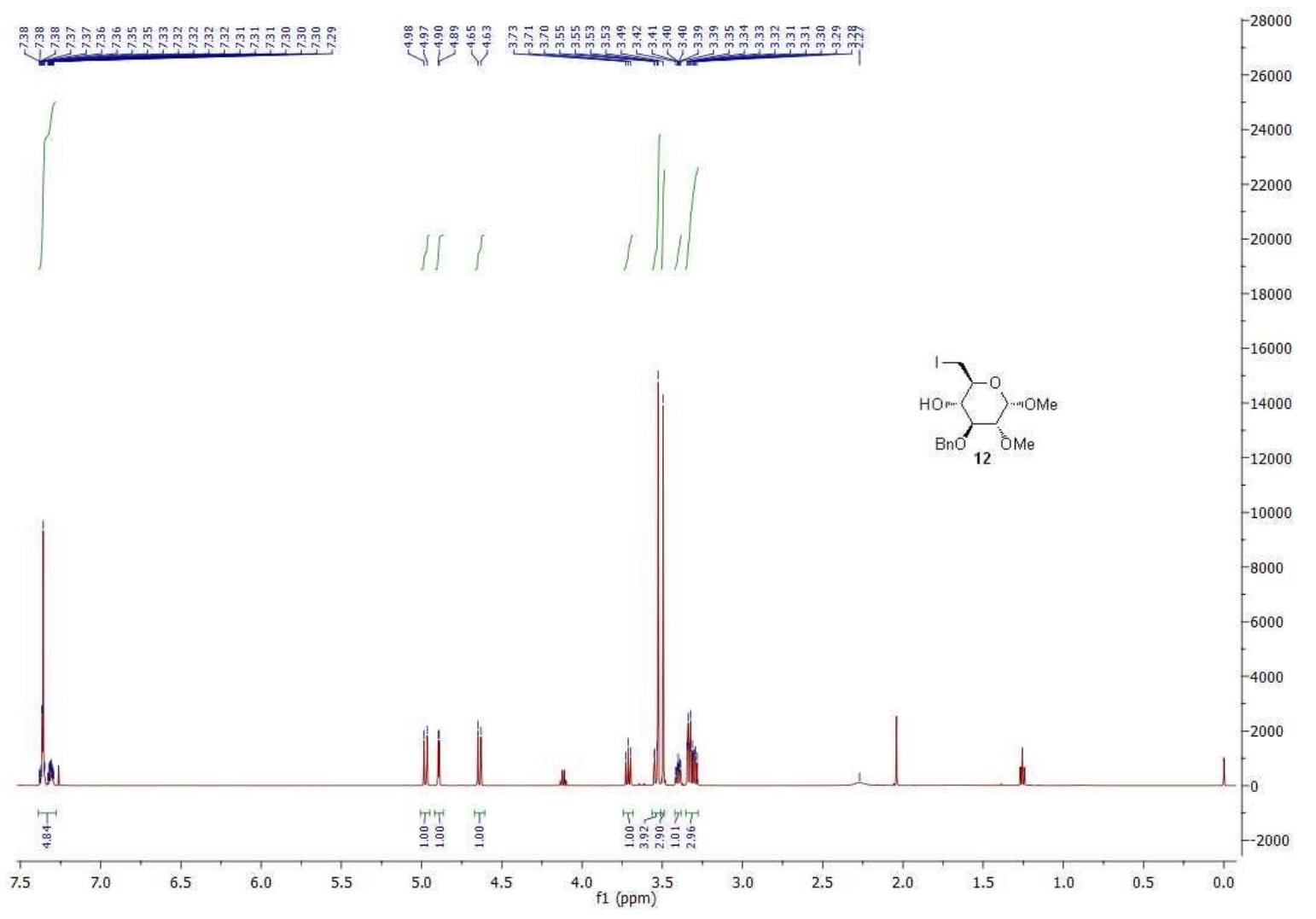

${ }^{1} \mathrm{H}-\mathrm{NMR}$ Spectrum of Compound $12\left(\mathrm{CDCl}_{3}\right)$

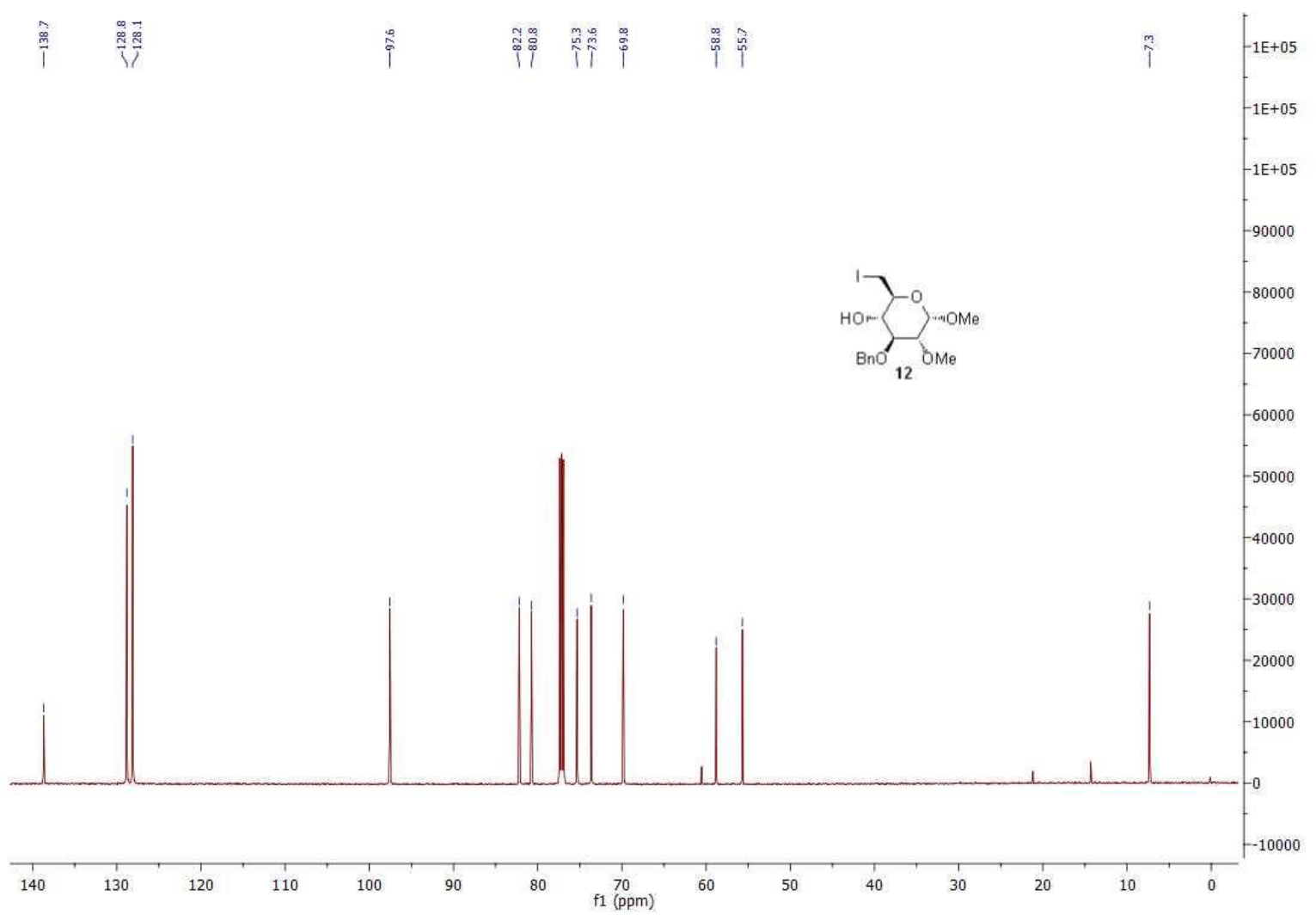

${ }^{13} \mathrm{C}$-NMR Spectrum of Compound $\mathbf{1 2}\left(\mathrm{CDCl}_{3}\right)$ 


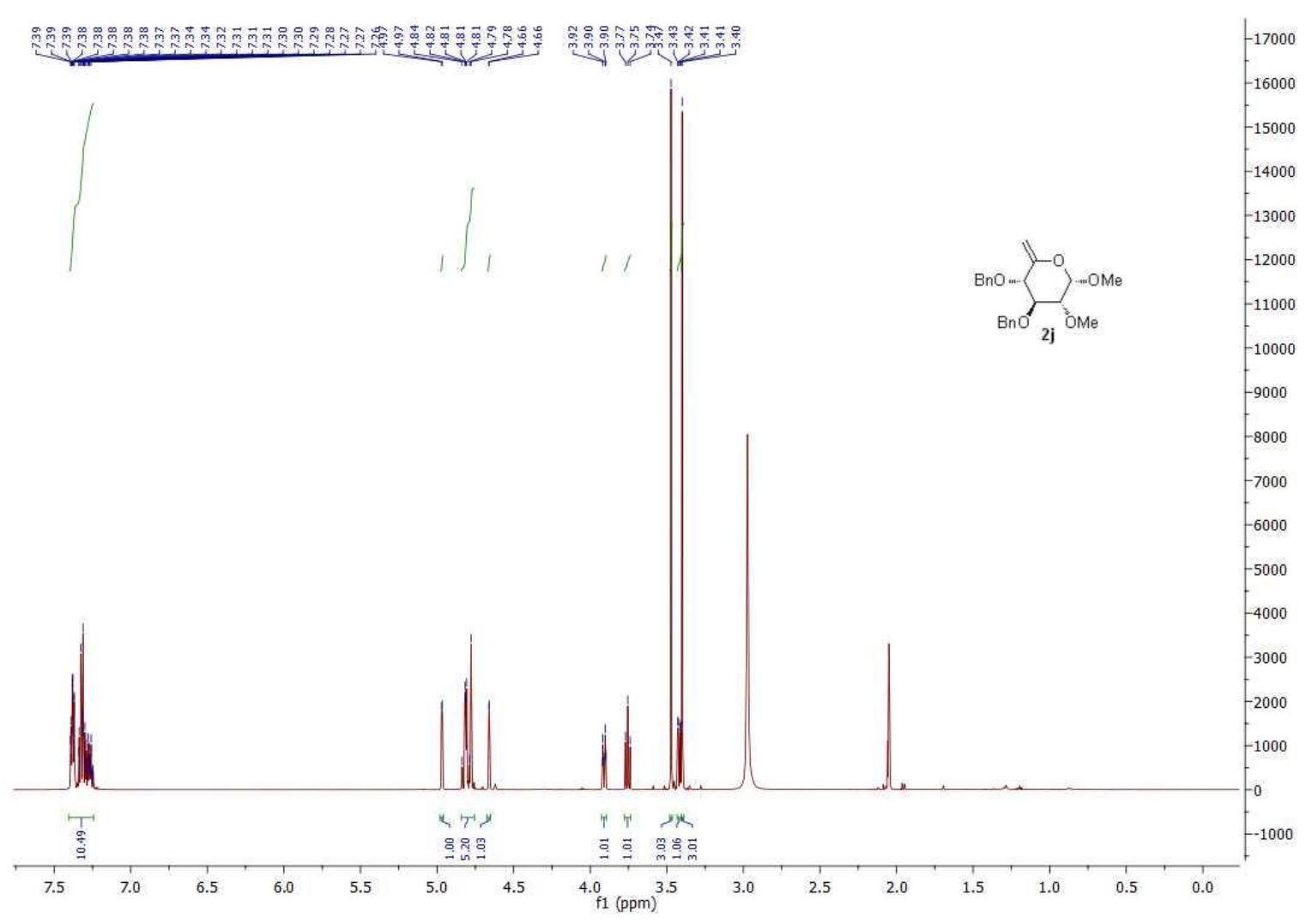

${ }^{1} \mathrm{H}-\mathrm{NMR}$ Spectrum of Compound $\mathbf{2 \mathbf { j }}$ (Acetone-d6)

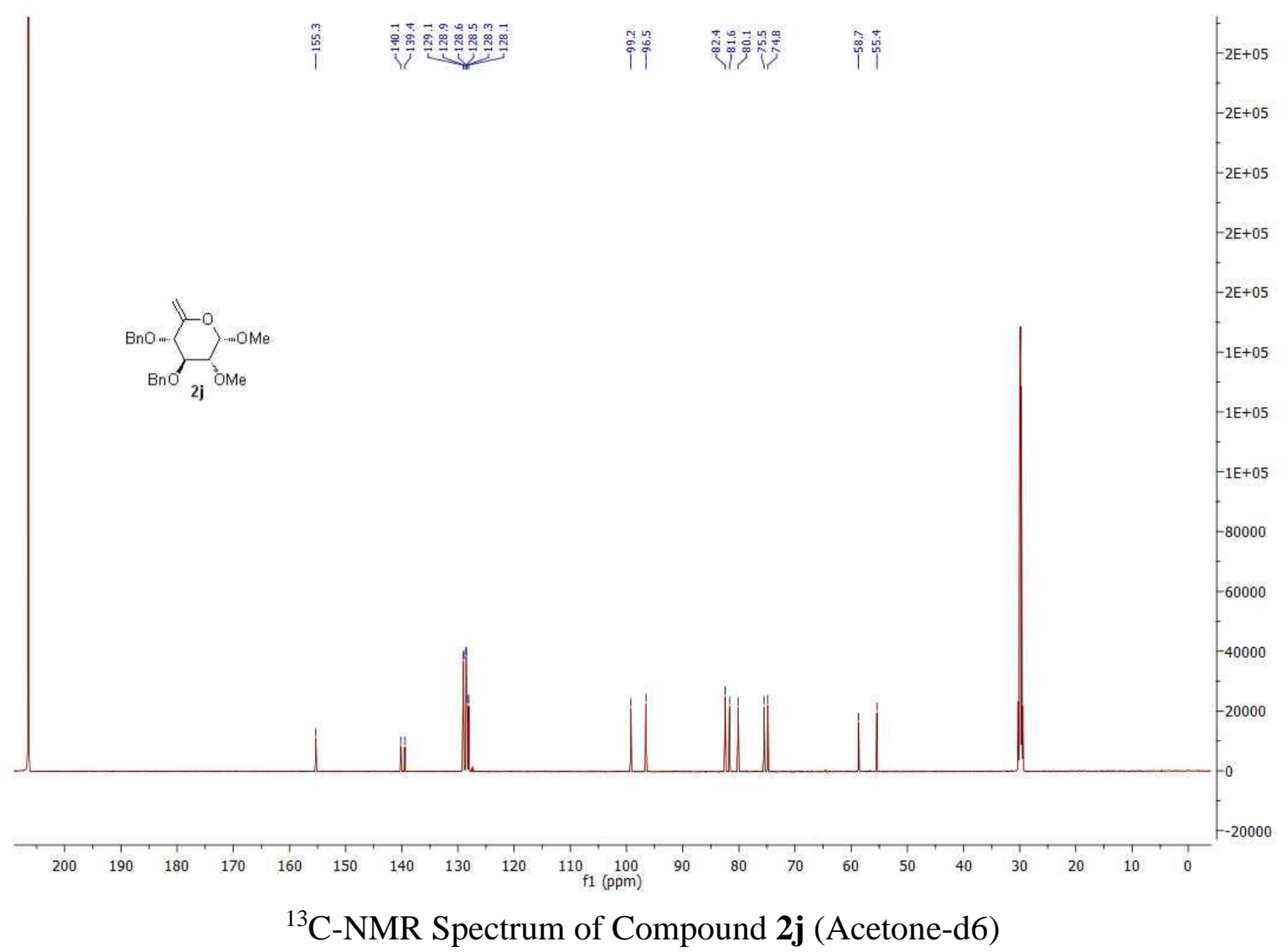




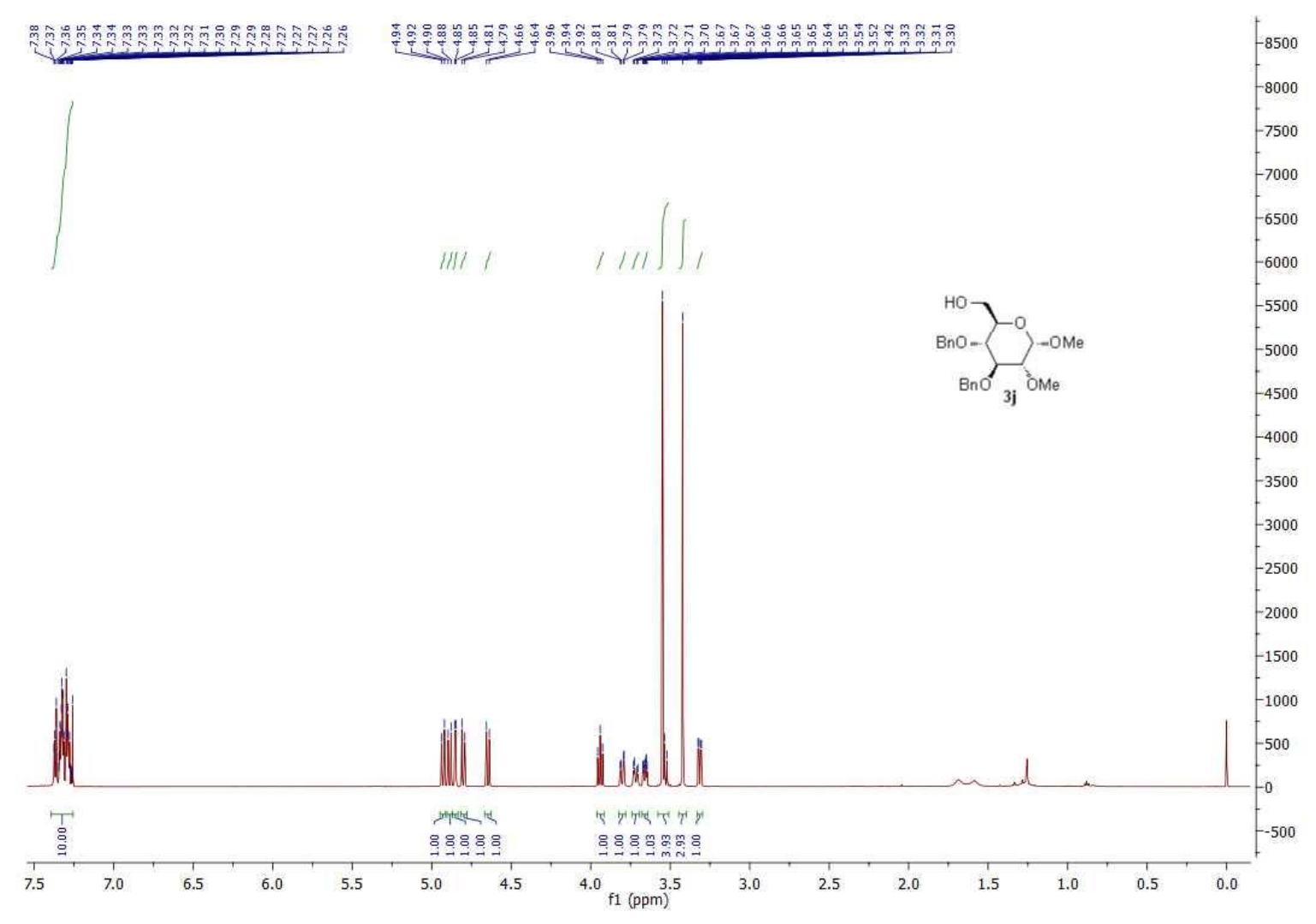

${ }^{1} \mathrm{H}-\mathrm{NMR}$ Spectrum of Compound $\mathbf{3 j}\left(\mathrm{CDCl}_{3}\right)$

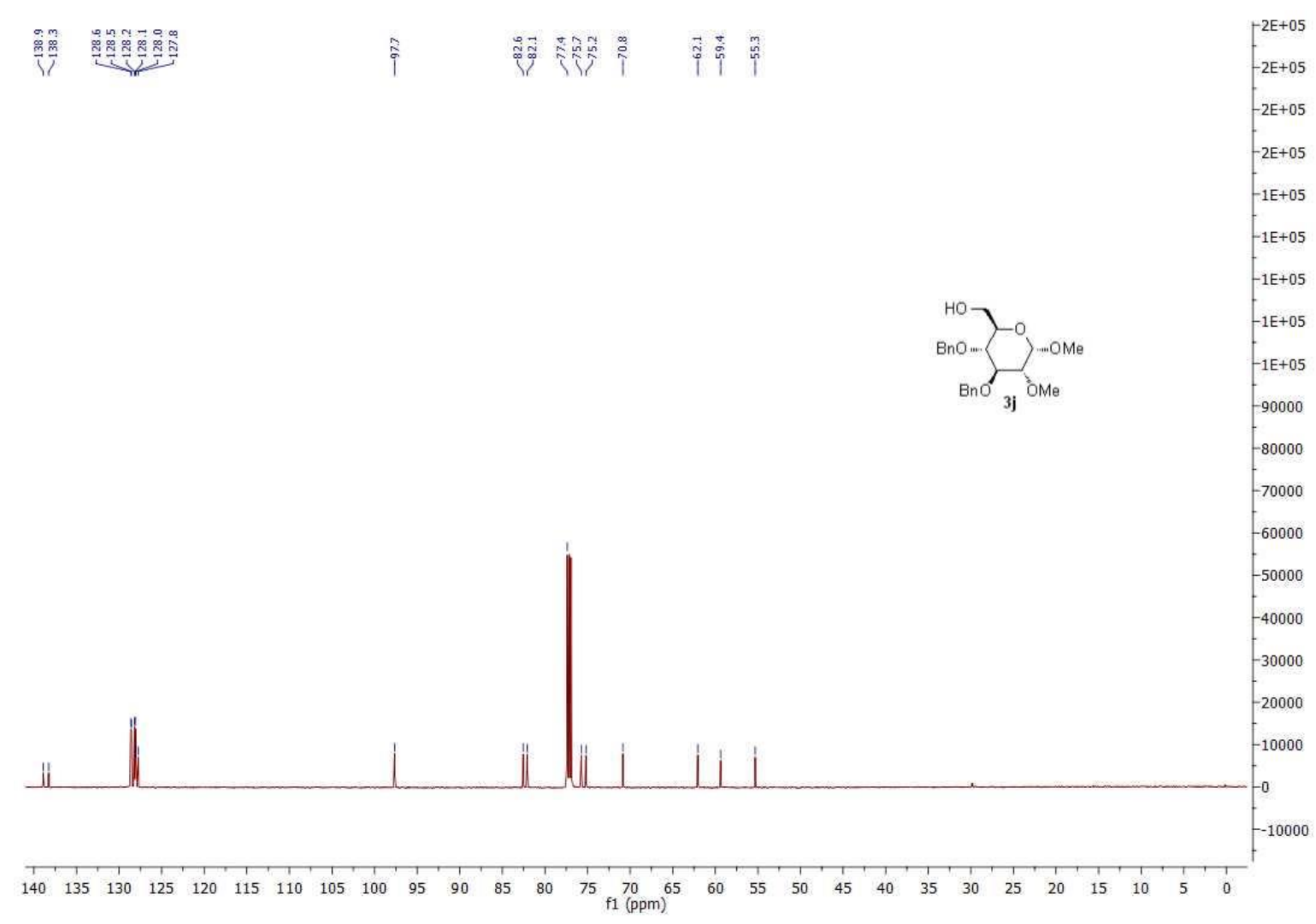

${ }^{13} \mathrm{C}-\mathrm{NMR}$ Spectrum of Compound $\mathbf{3 j}\left(\mathrm{CDCl}_{3}\right)$ 


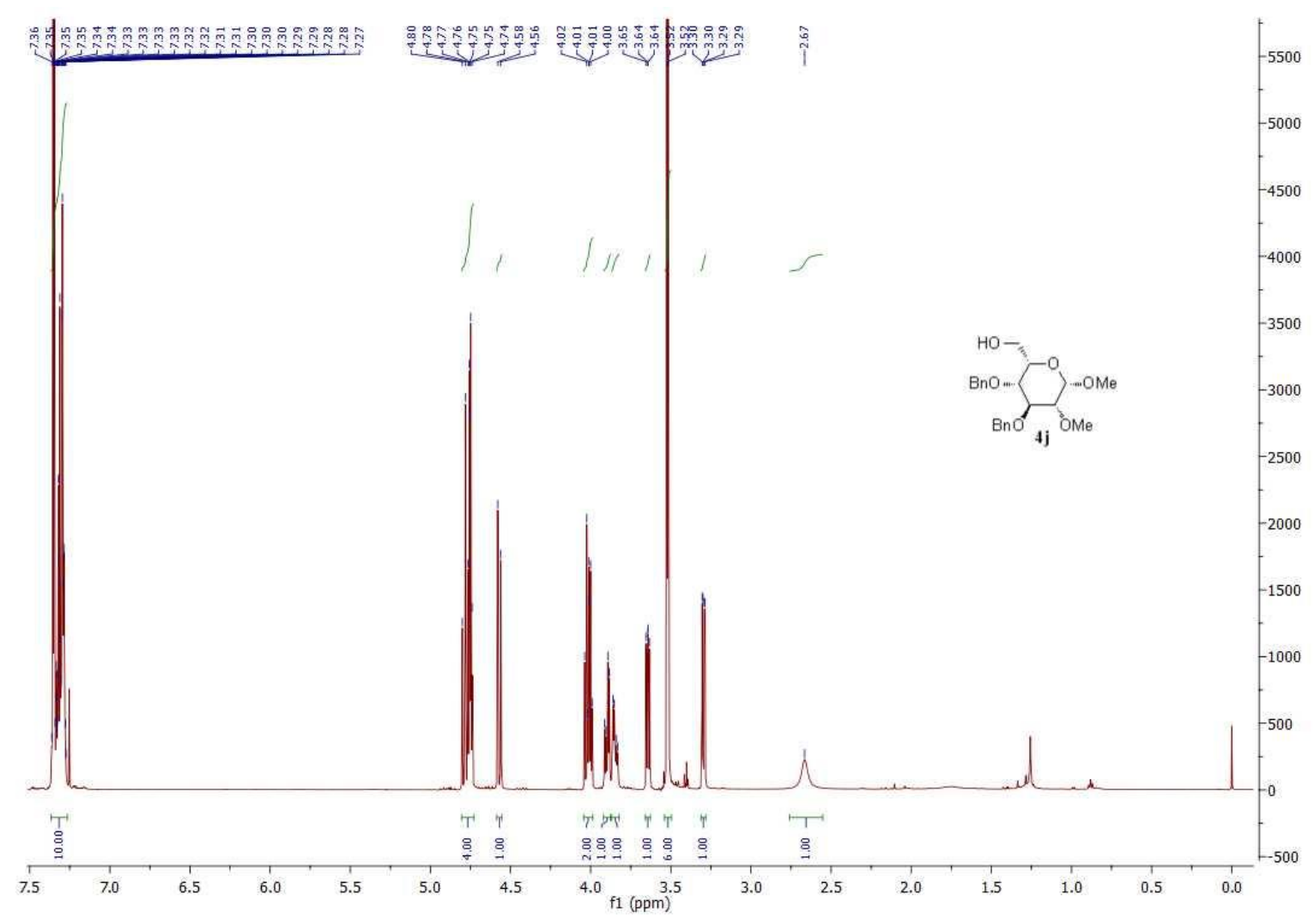

${ }^{1} \mathrm{H}-\mathrm{NMR}$ Spectrum of Compound $\mathbf{4} \mathbf{j}\left(\mathrm{CDCl}_{3}\right)$

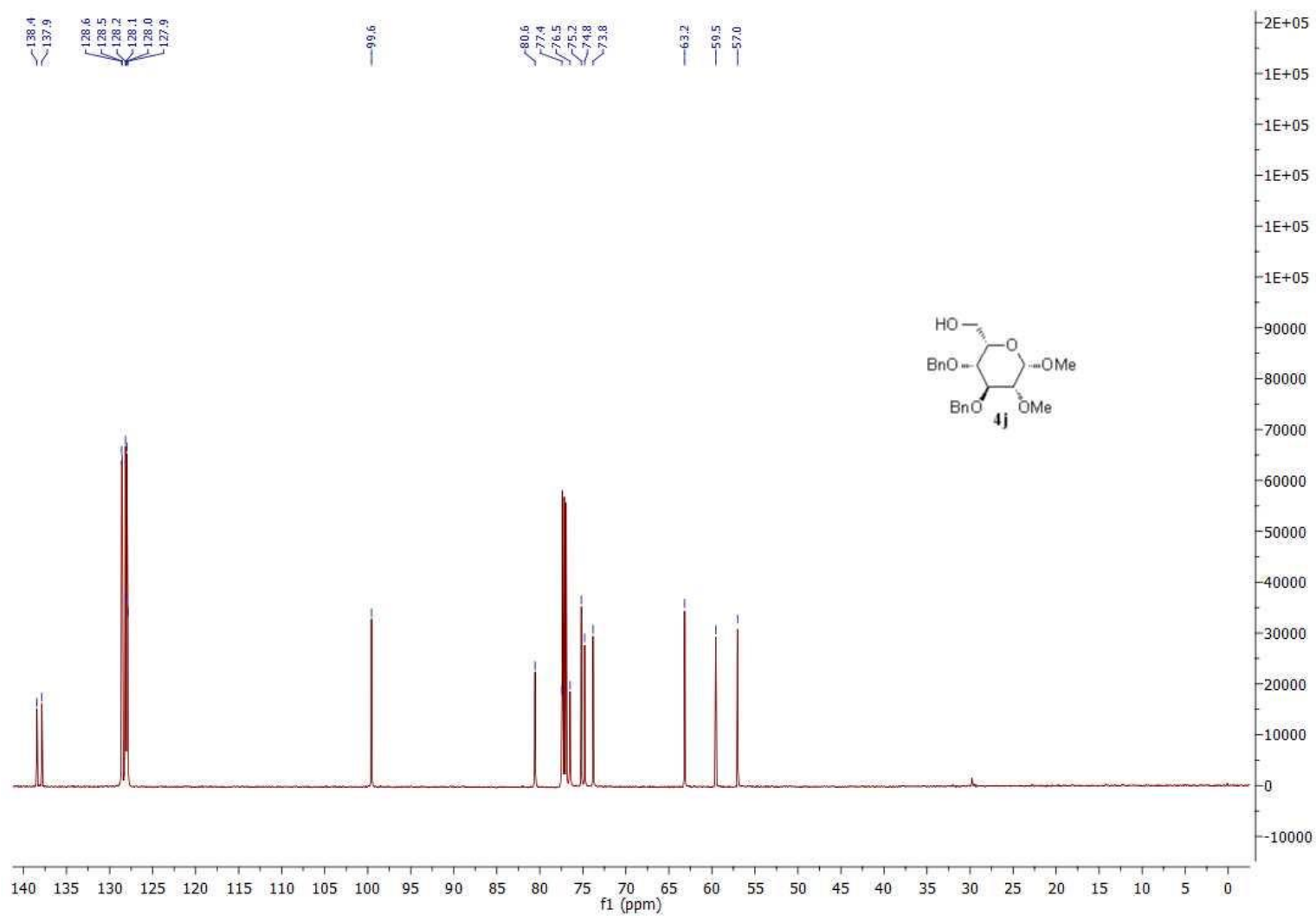

${ }^{13} \mathrm{C}-\mathrm{NMR}$ Spectrum of Compound $\mathbf{4 j}\left(\mathrm{CDCl}_{3}\right)$ 


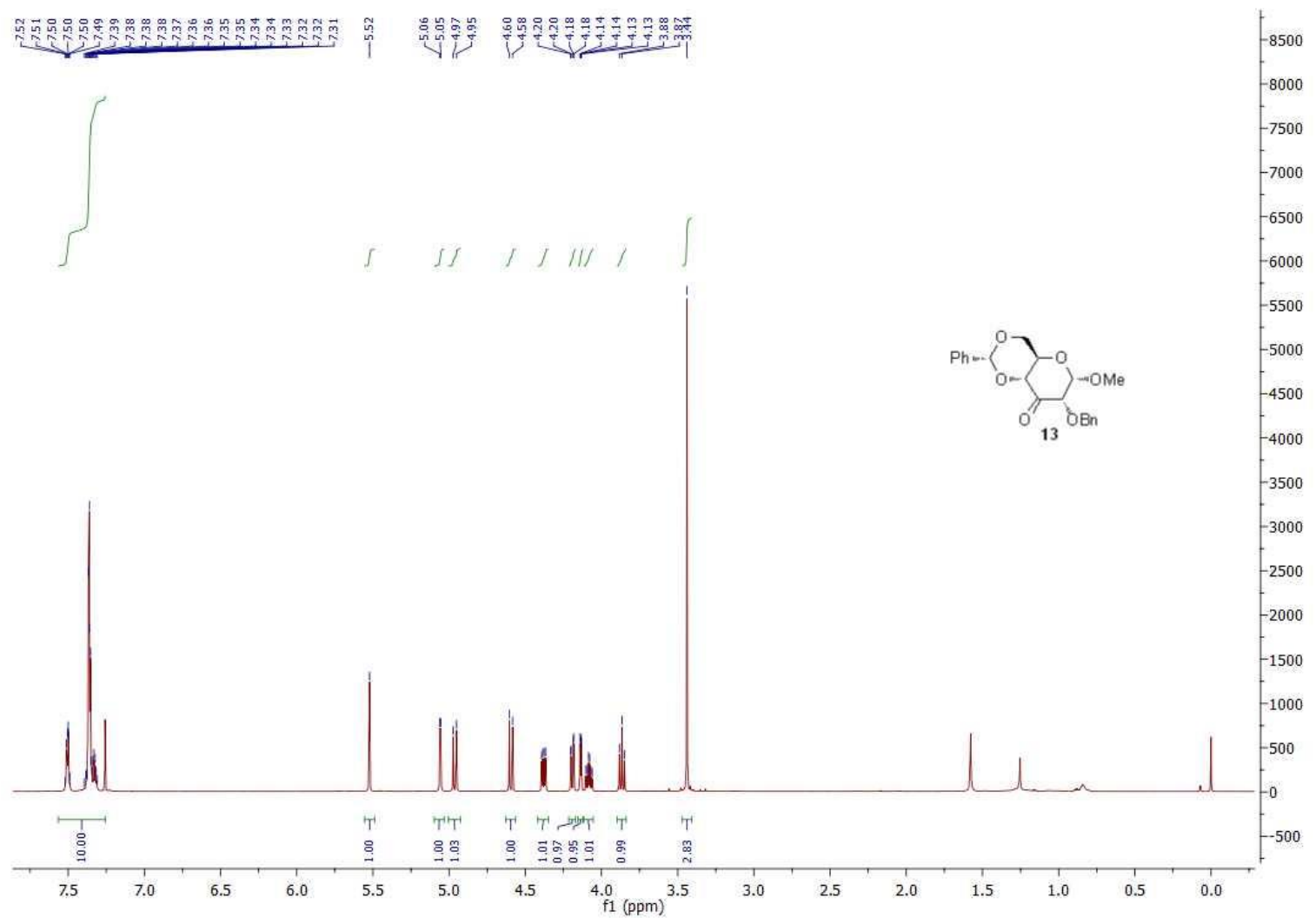

${ }^{1} \mathrm{H}-\mathrm{NMR}$ Spectrum of Compound $\mathbf{1 3}\left(\mathrm{CDCl}_{3}\right)$

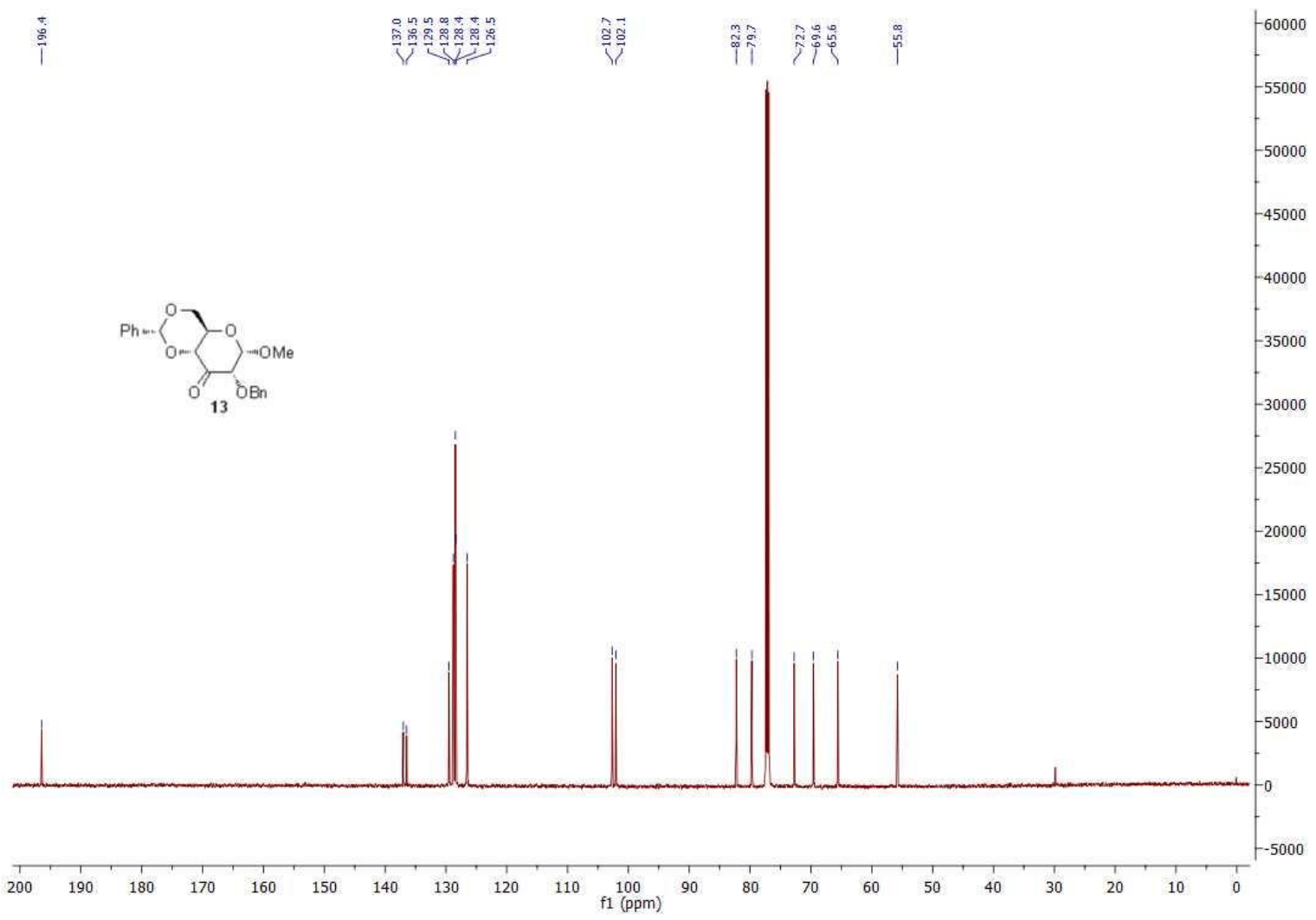

${ }^{13} \mathrm{C}$-NMR Spectrum of Compound $\mathbf{1 3}\left(\mathrm{CDCl}_{3}\right)$ 


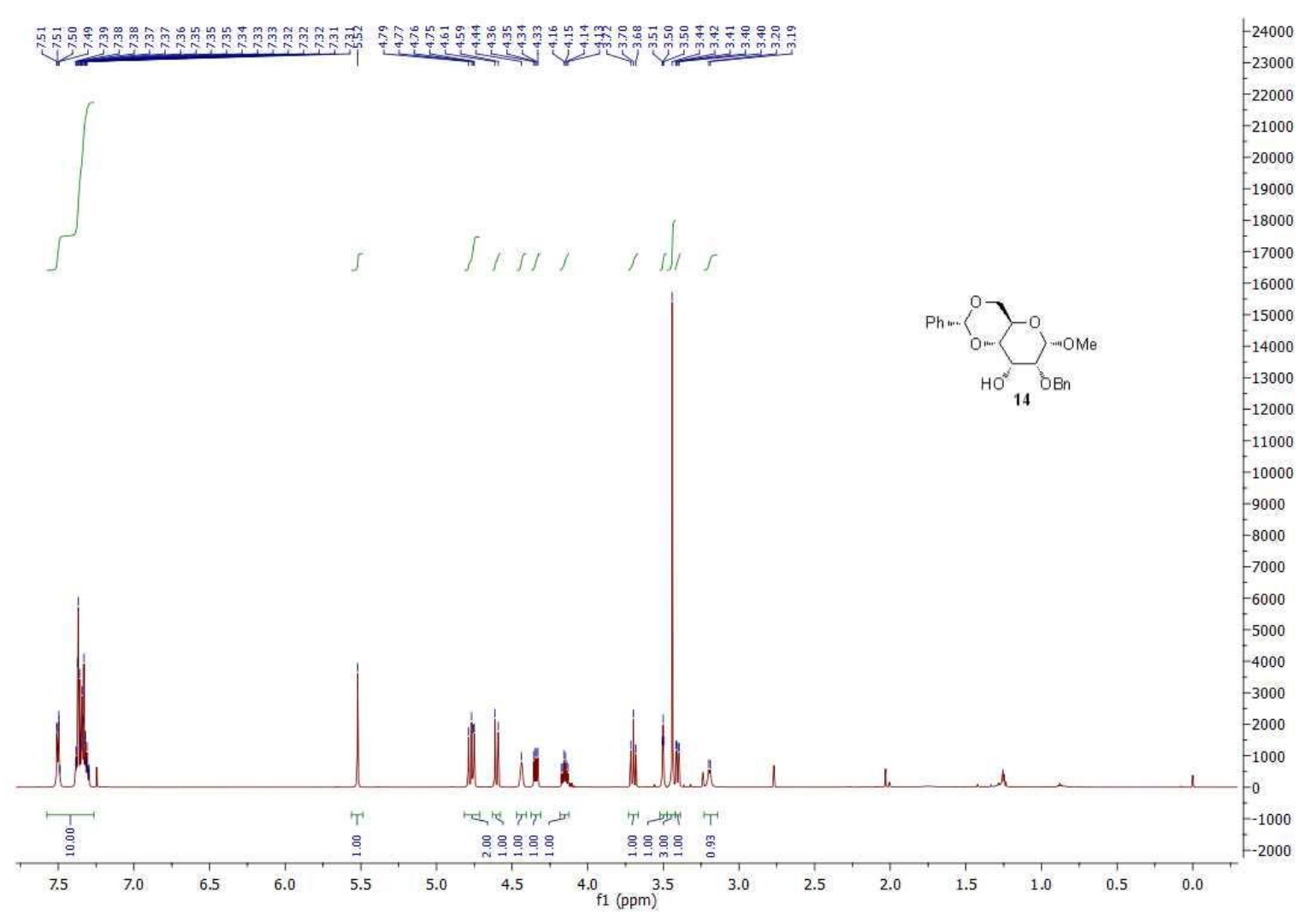

${ }^{1} \mathrm{H}-\mathrm{NMR}$ Spectrum of Compound $14\left(\mathrm{CDCl}_{3}\right)$

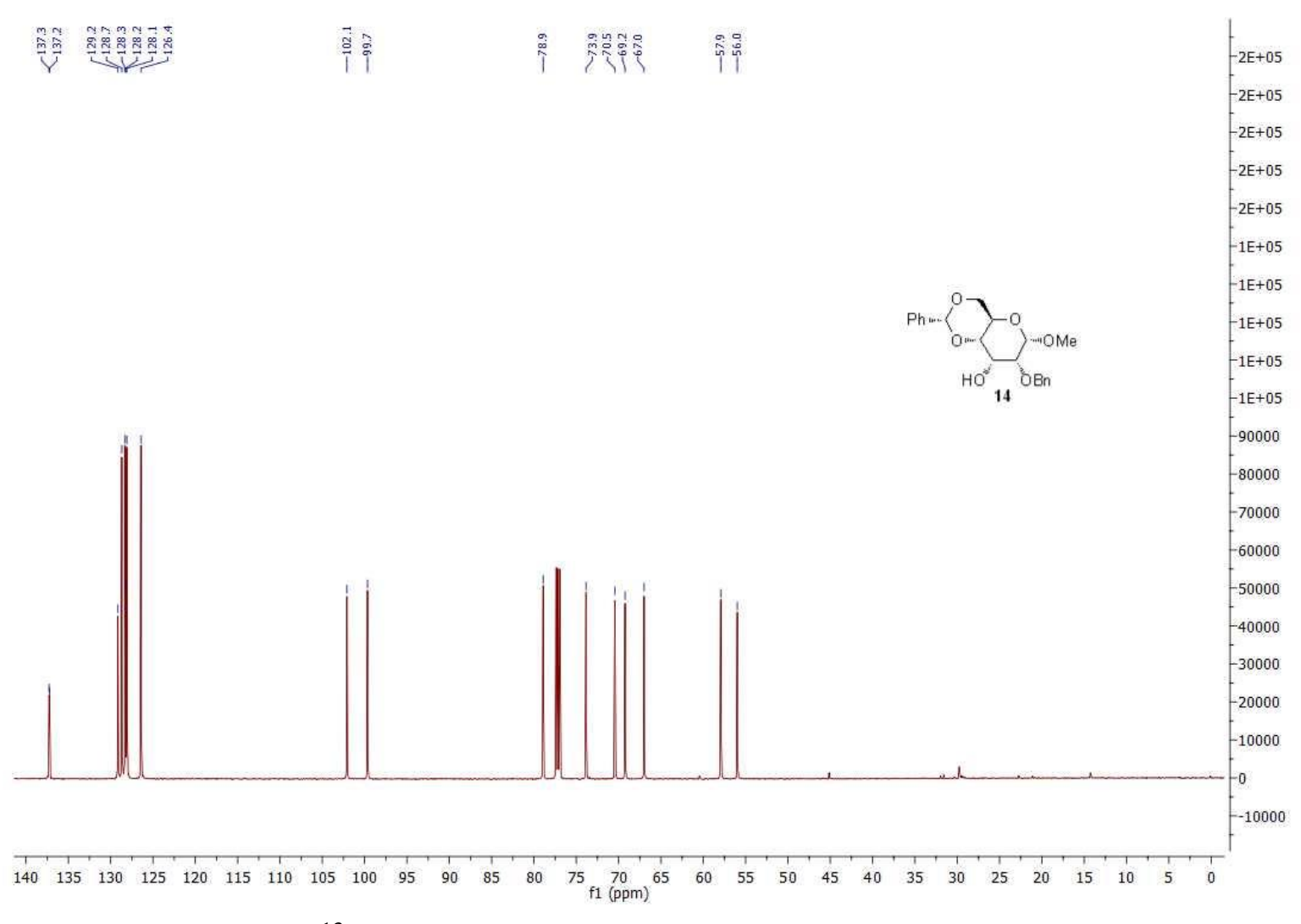

${ }^{13} \mathrm{C}-\mathrm{NMR}$ Spectrum of Compound $\mathbf{1 4}\left(\mathrm{CDCl}_{3}\right)$ 


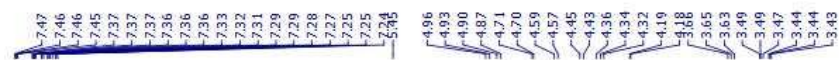

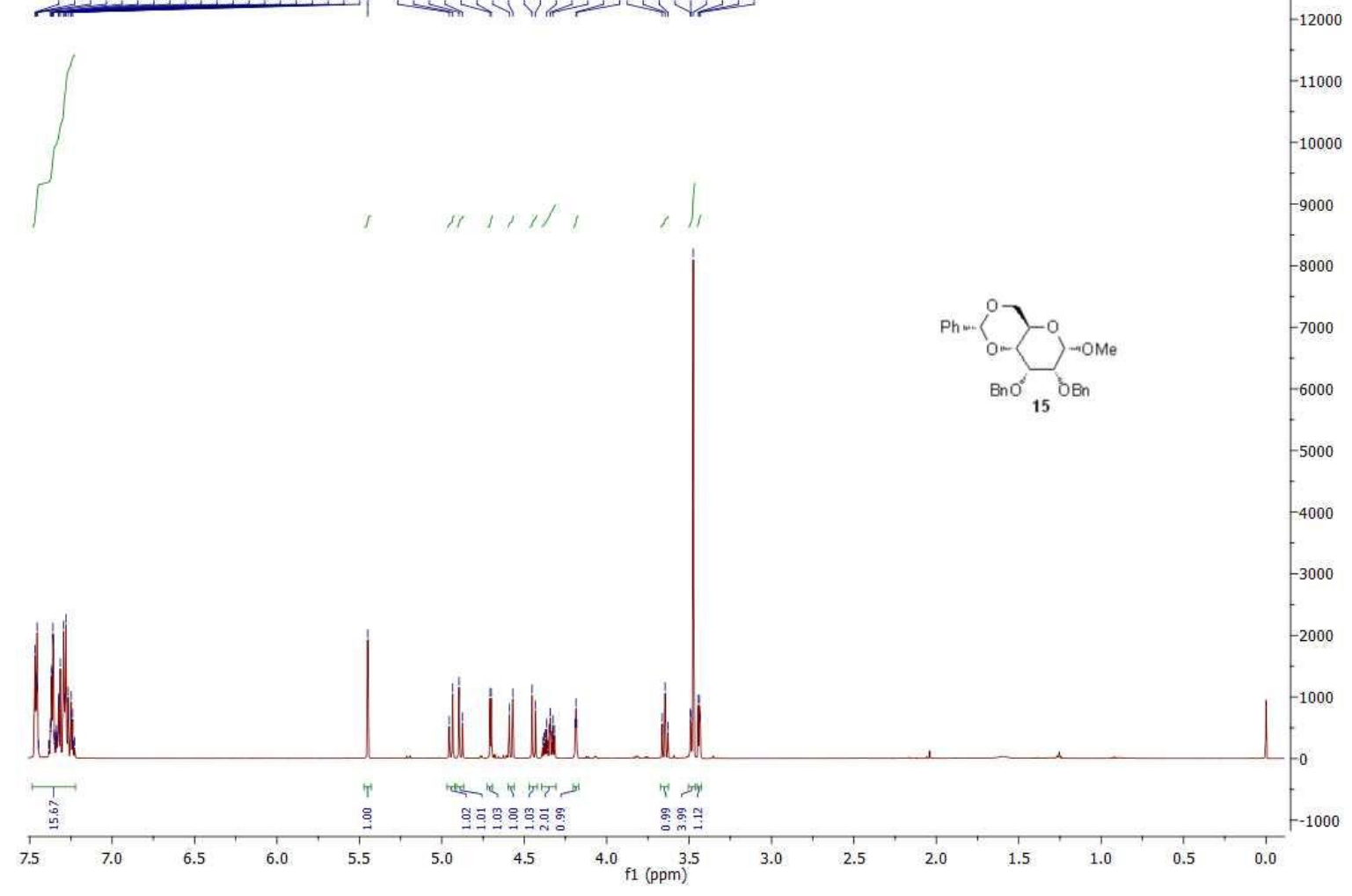

${ }^{1} \mathrm{H}-\mathrm{NMR}$ Spectrum of Compound $15\left(\mathrm{CDCl}_{3}\right)$

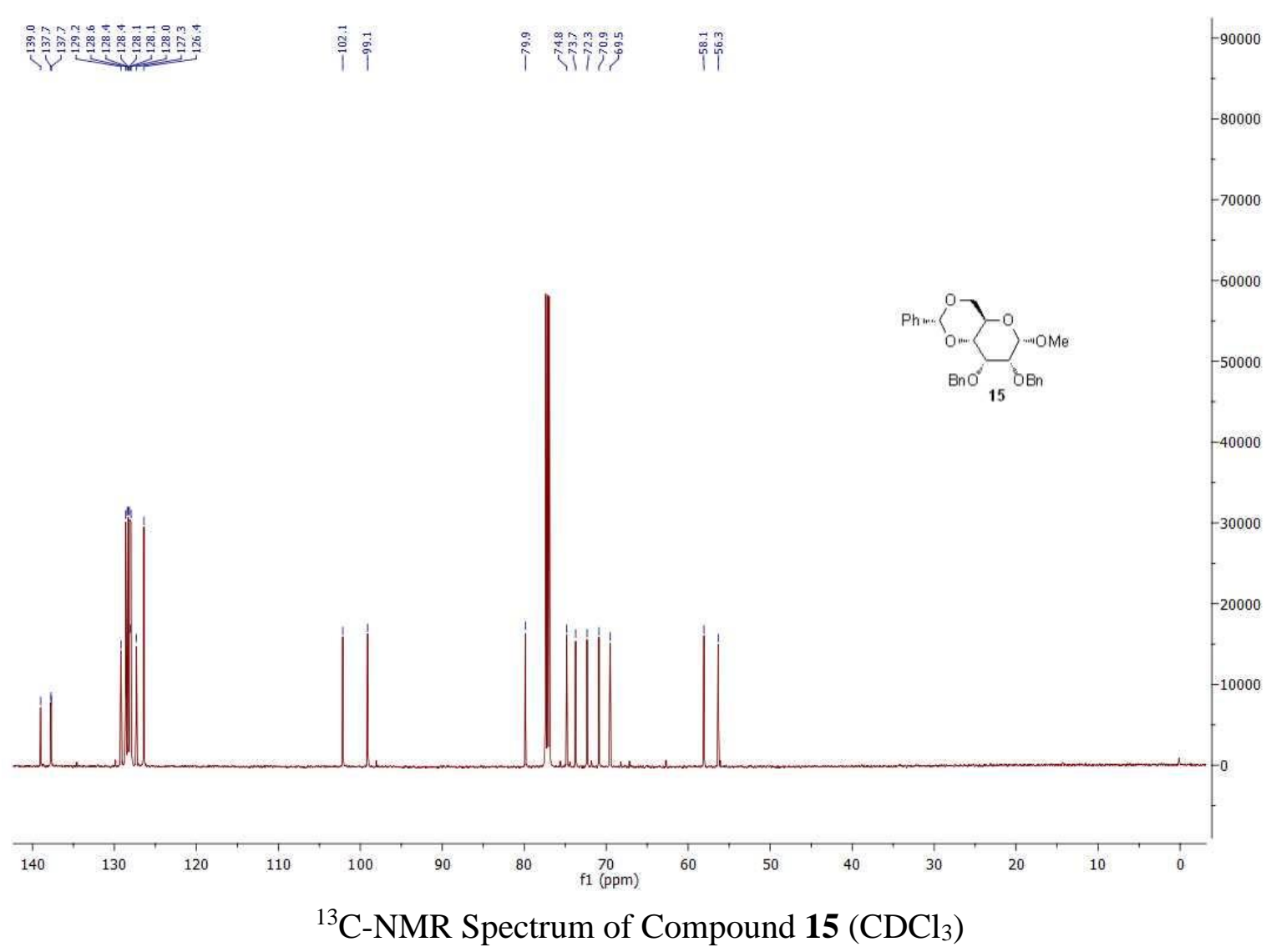




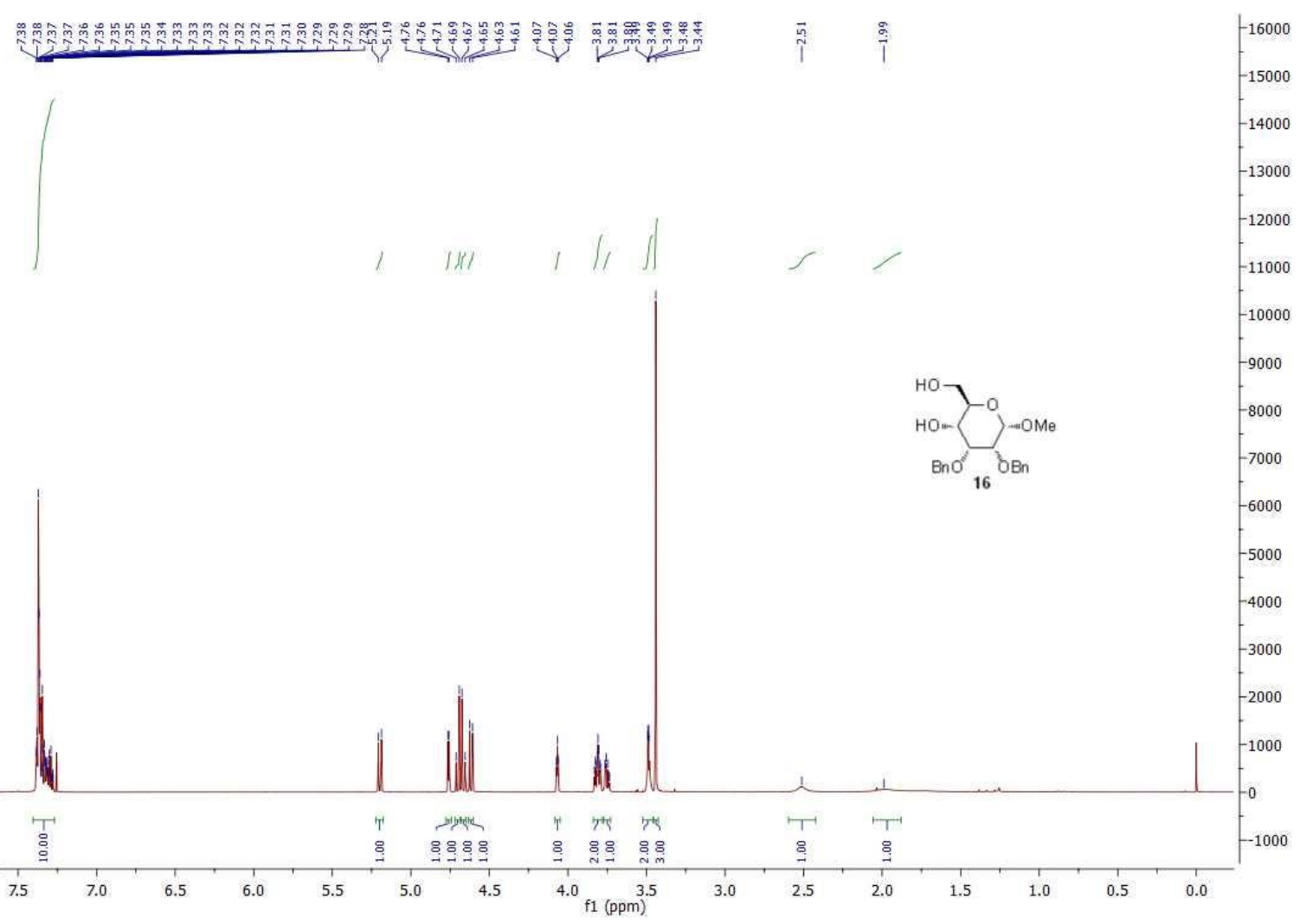

${ }^{1} \mathrm{H}-\mathrm{NMR}$ Spectrum of Compound $16\left(\mathrm{CDCl}_{3}\right)$

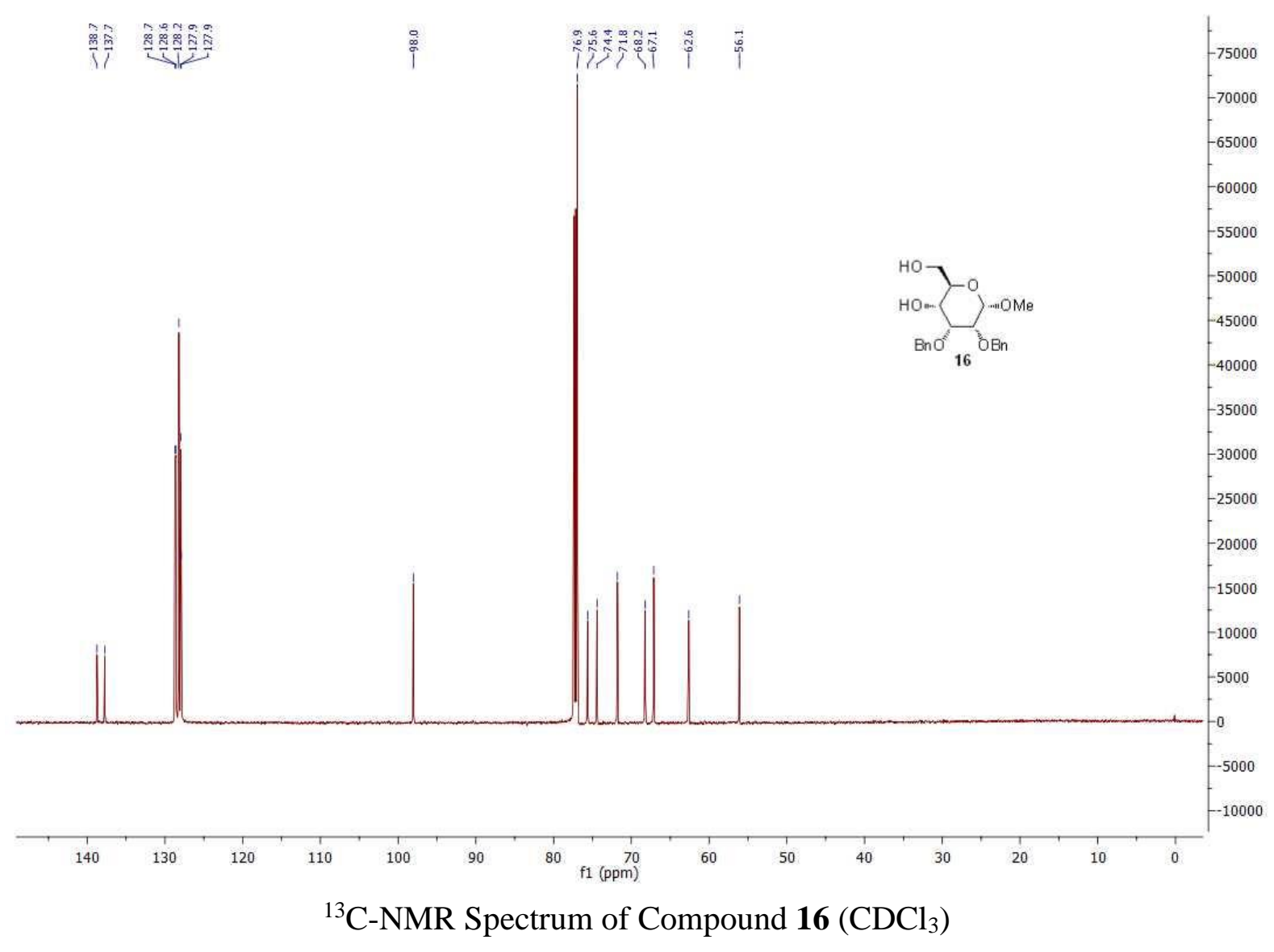




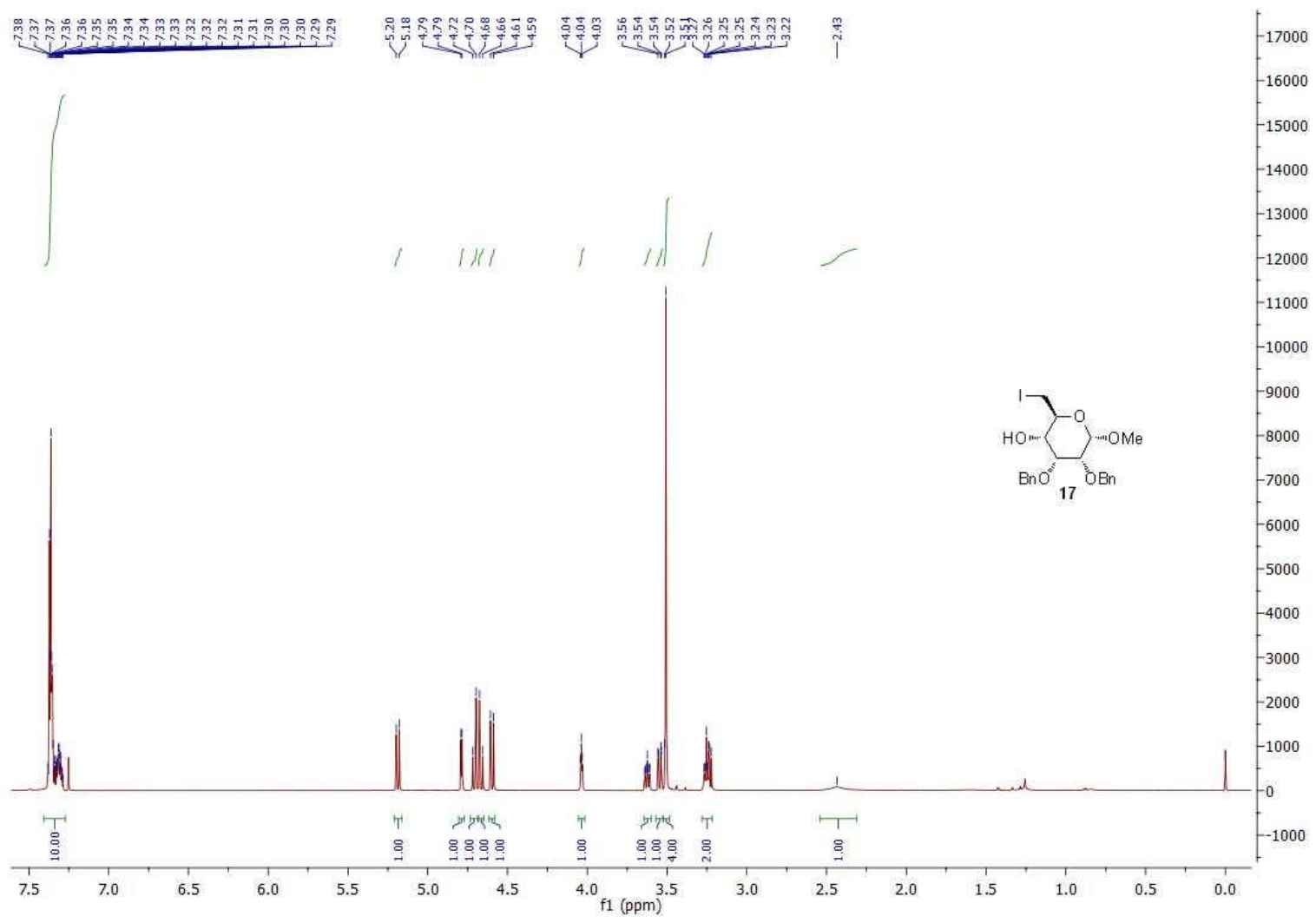

${ }^{1} \mathrm{H}-\mathrm{NMR}$ Spectrum of Compound $17\left(\mathrm{CDCl}_{3}\right)$

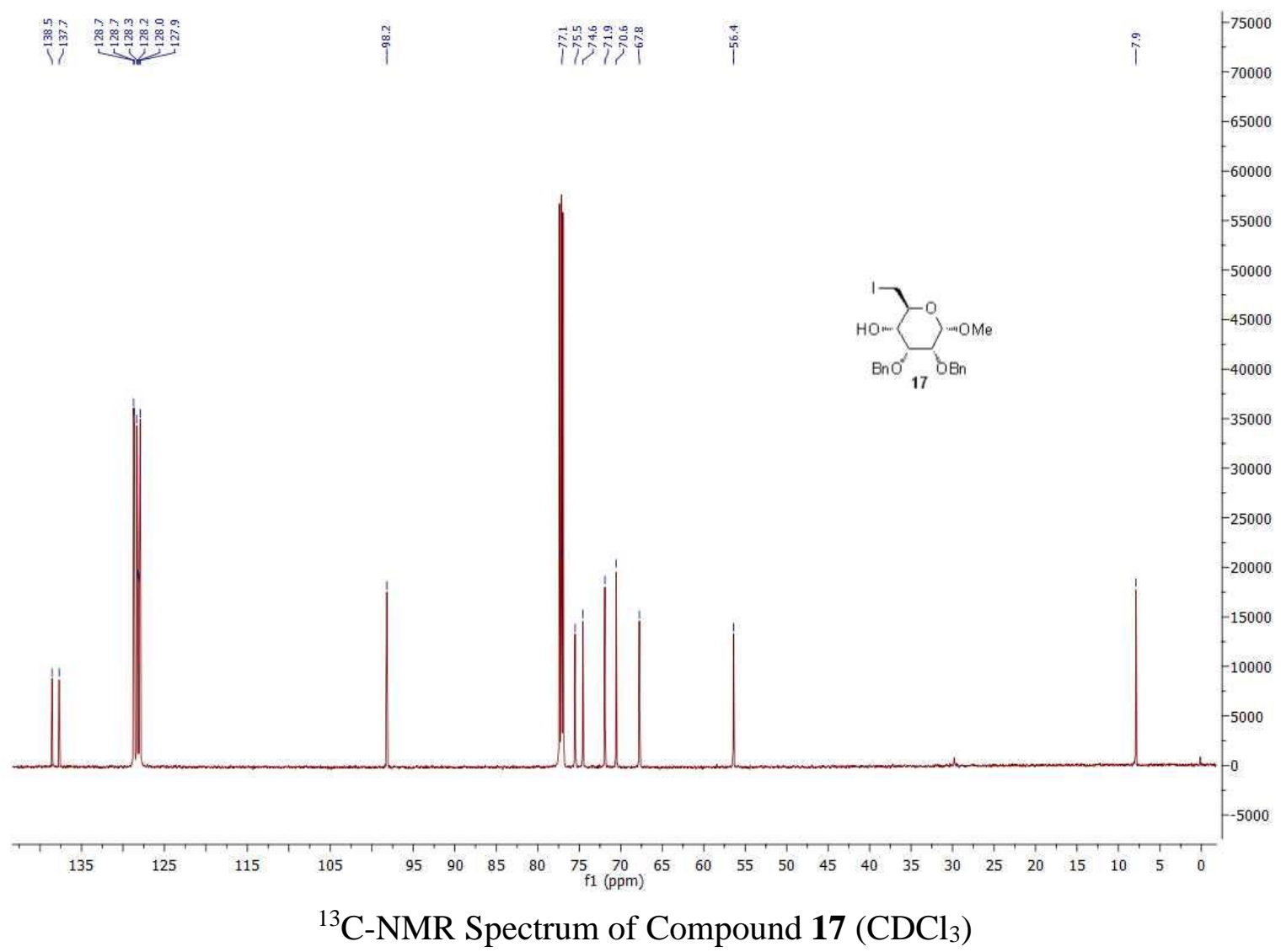




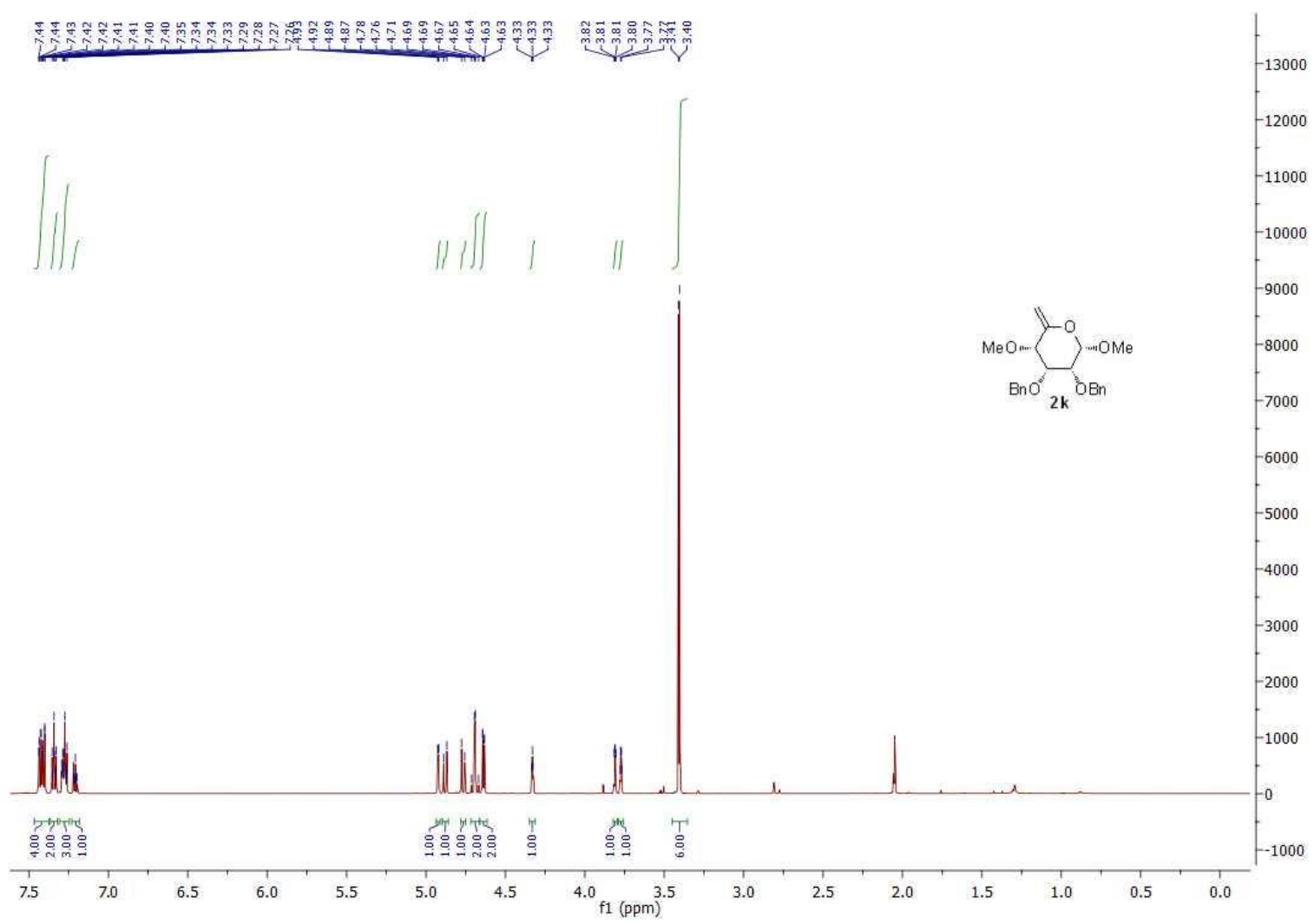

${ }^{1} \mathrm{H}-\mathrm{NMR}$ Spectrum of Compound 2k (Acetone-d6)

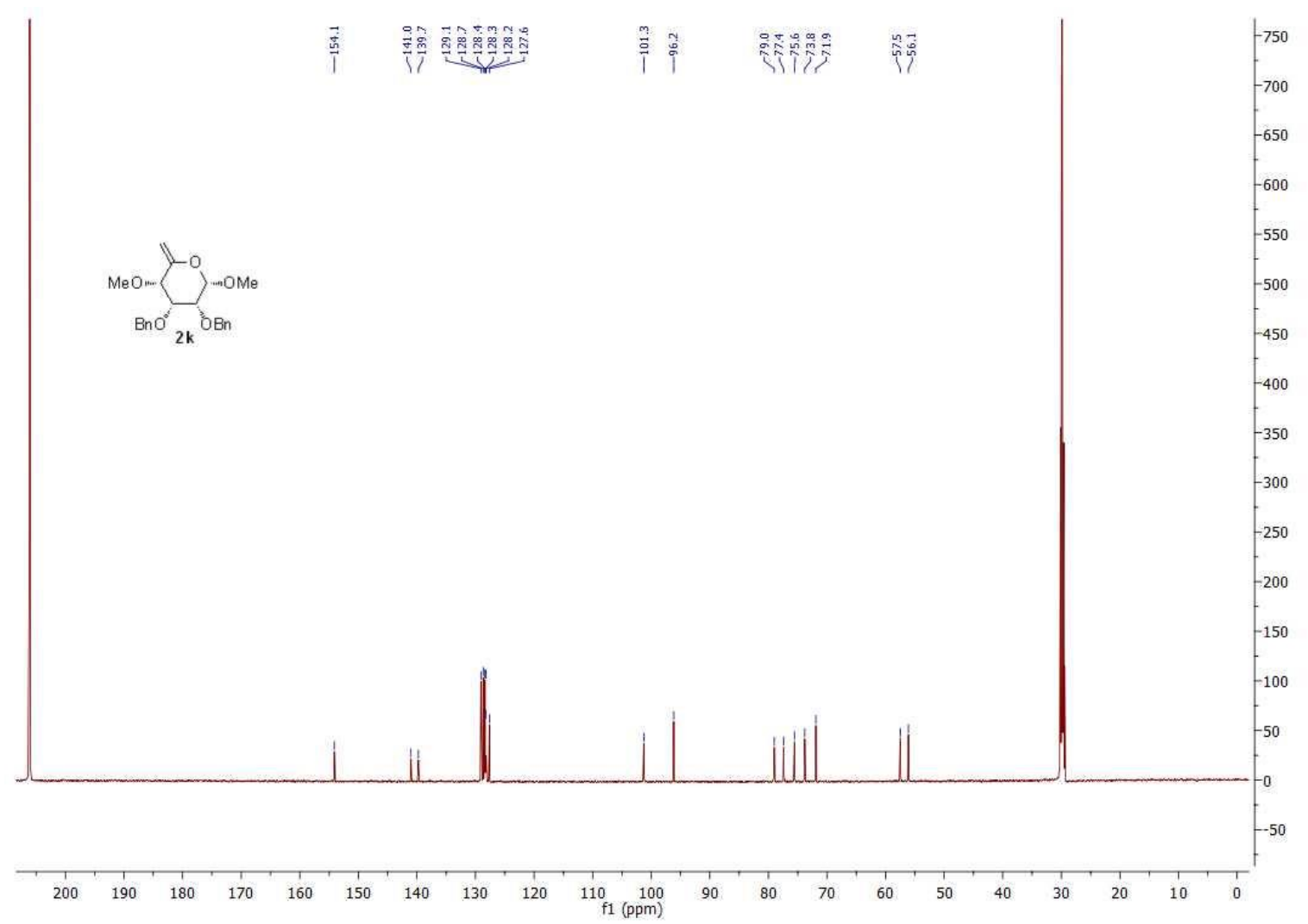

${ }^{13} \mathrm{C}-\mathrm{NMR}$ Spectrum of Compound 2k (Acetone-d6) 


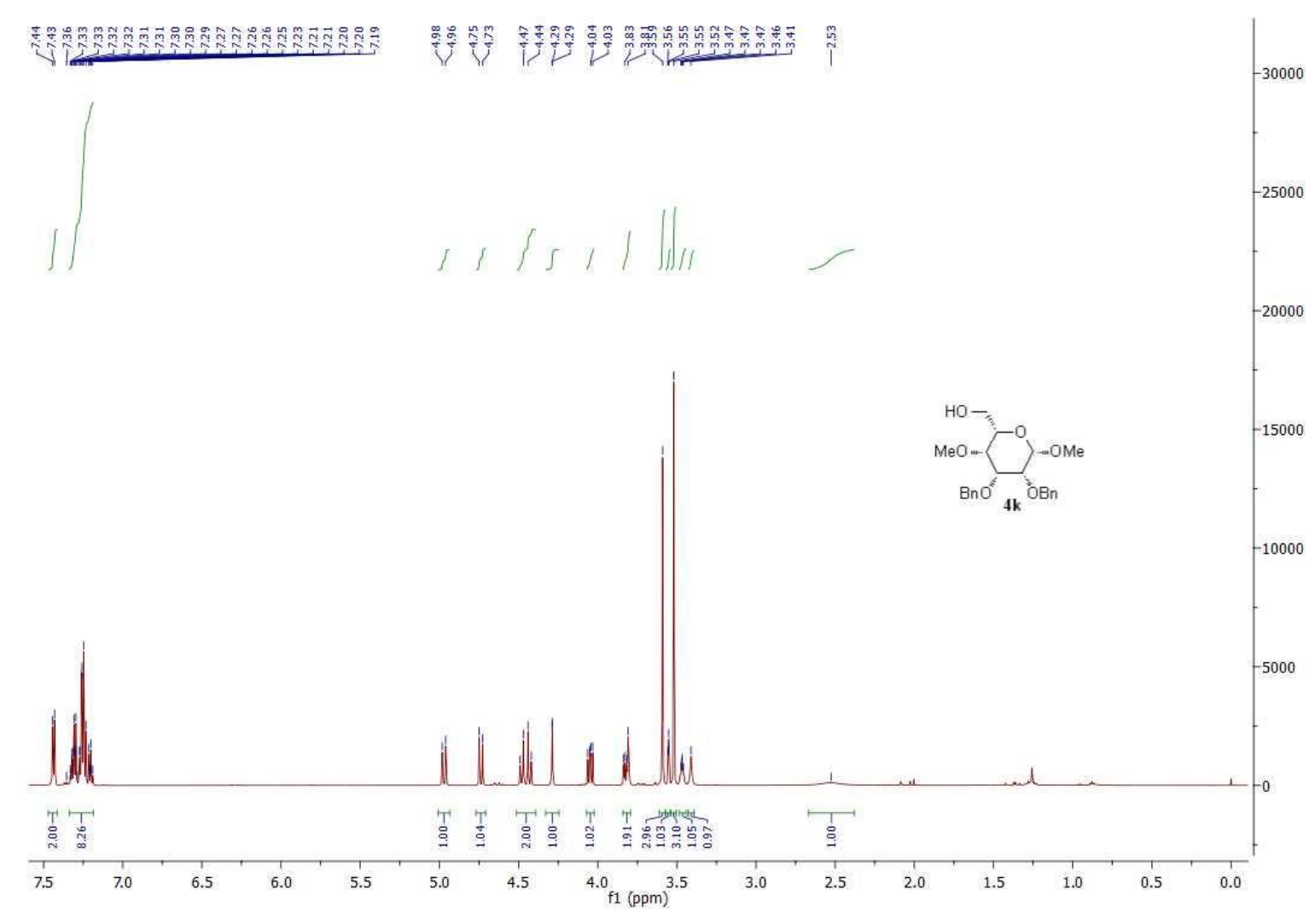

${ }^{1} \mathrm{H}-\mathrm{NMR}$ Spectrum of Compound $\mathbf{4 k}\left(\mathrm{CDCl}_{3}\right)$

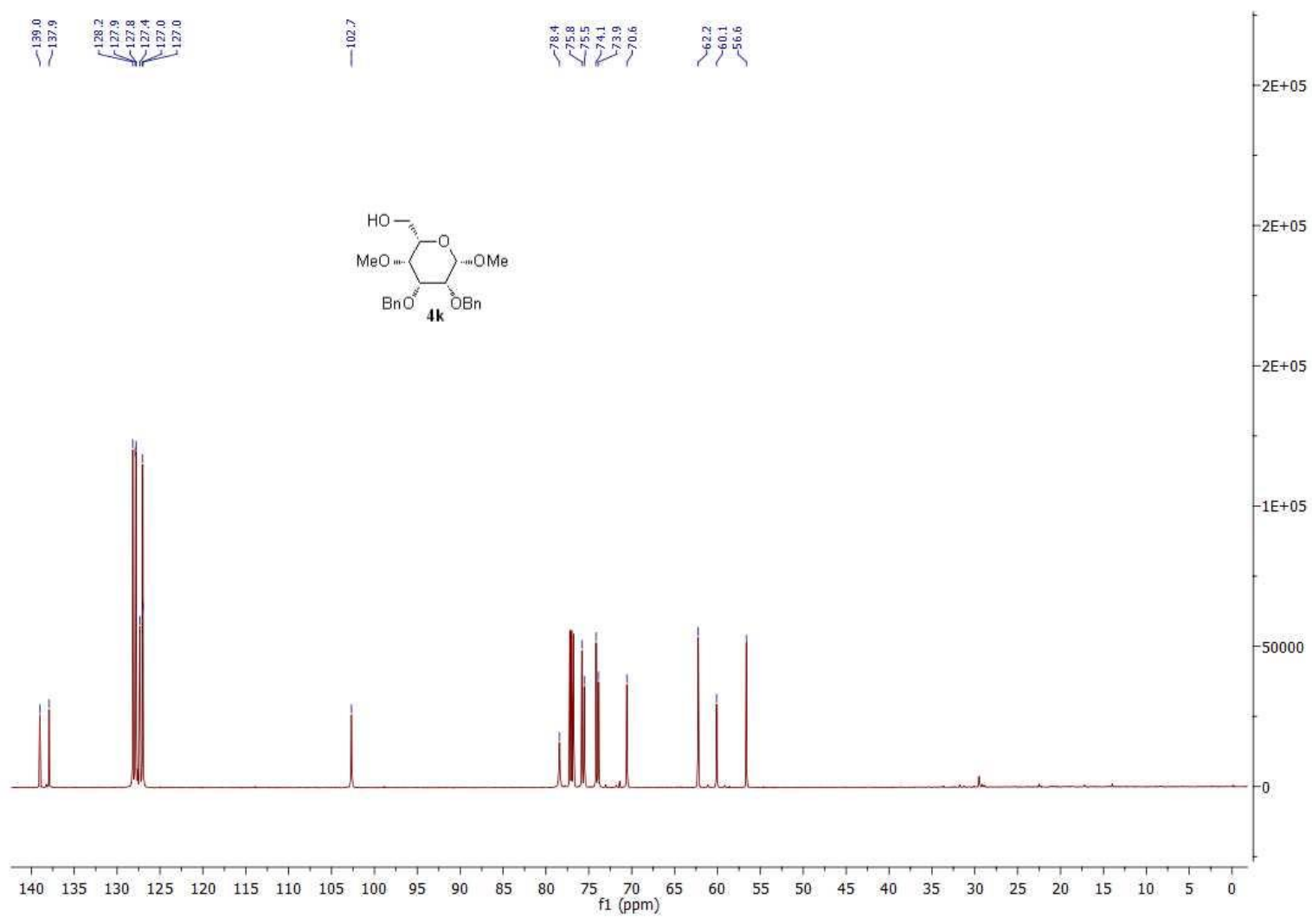

${ }^{13} \mathrm{C}-\mathrm{NMR}$ Spectrum of Compound $\mathbf{4 k}\left(\mathrm{CDCl}_{3}\right)$ 


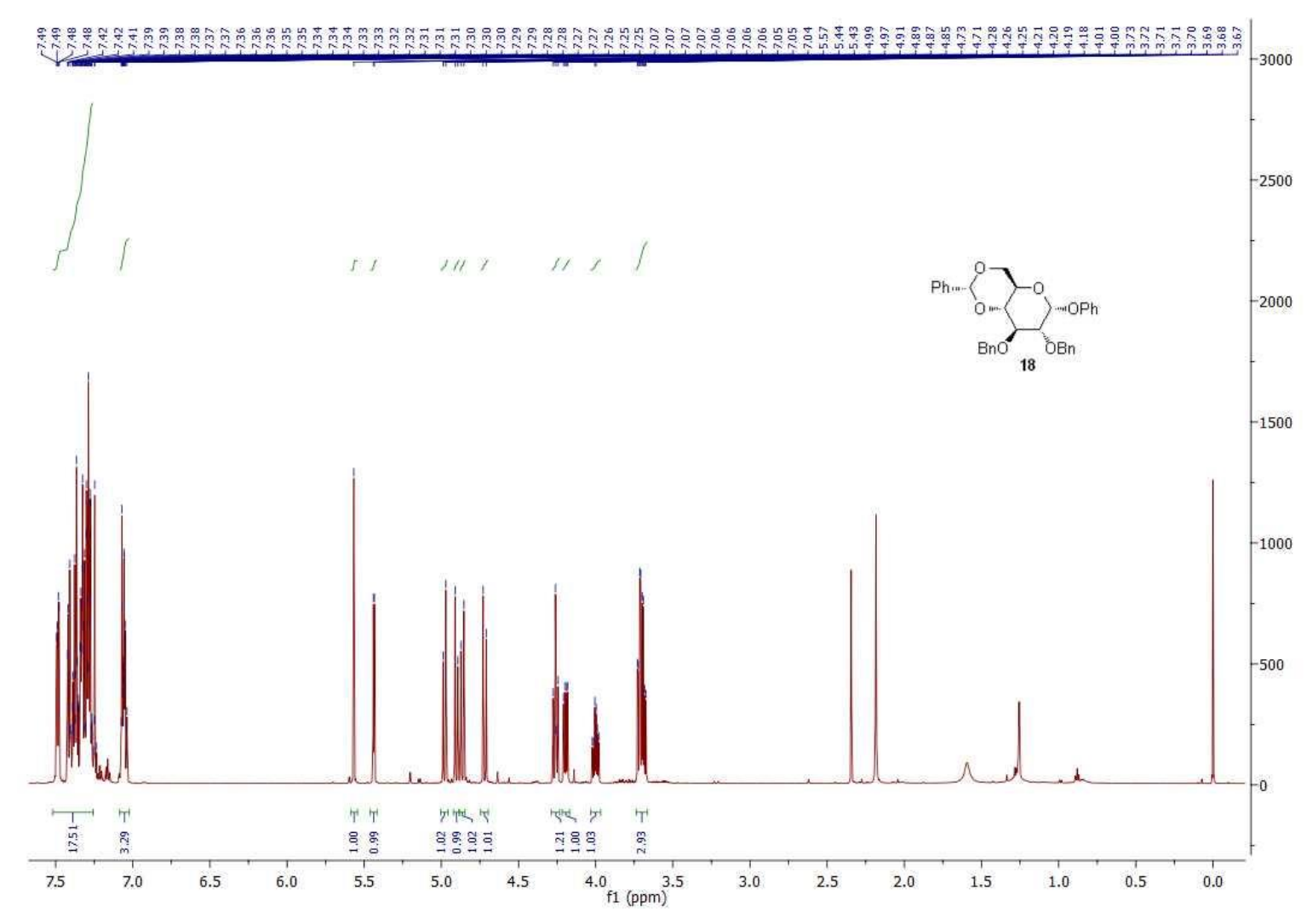

${ }^{1} \mathrm{H}-\mathrm{NMR}$ Spectrum of Compound $\mathbf{1 8}\left(\mathrm{CDCl}_{3}\right)$

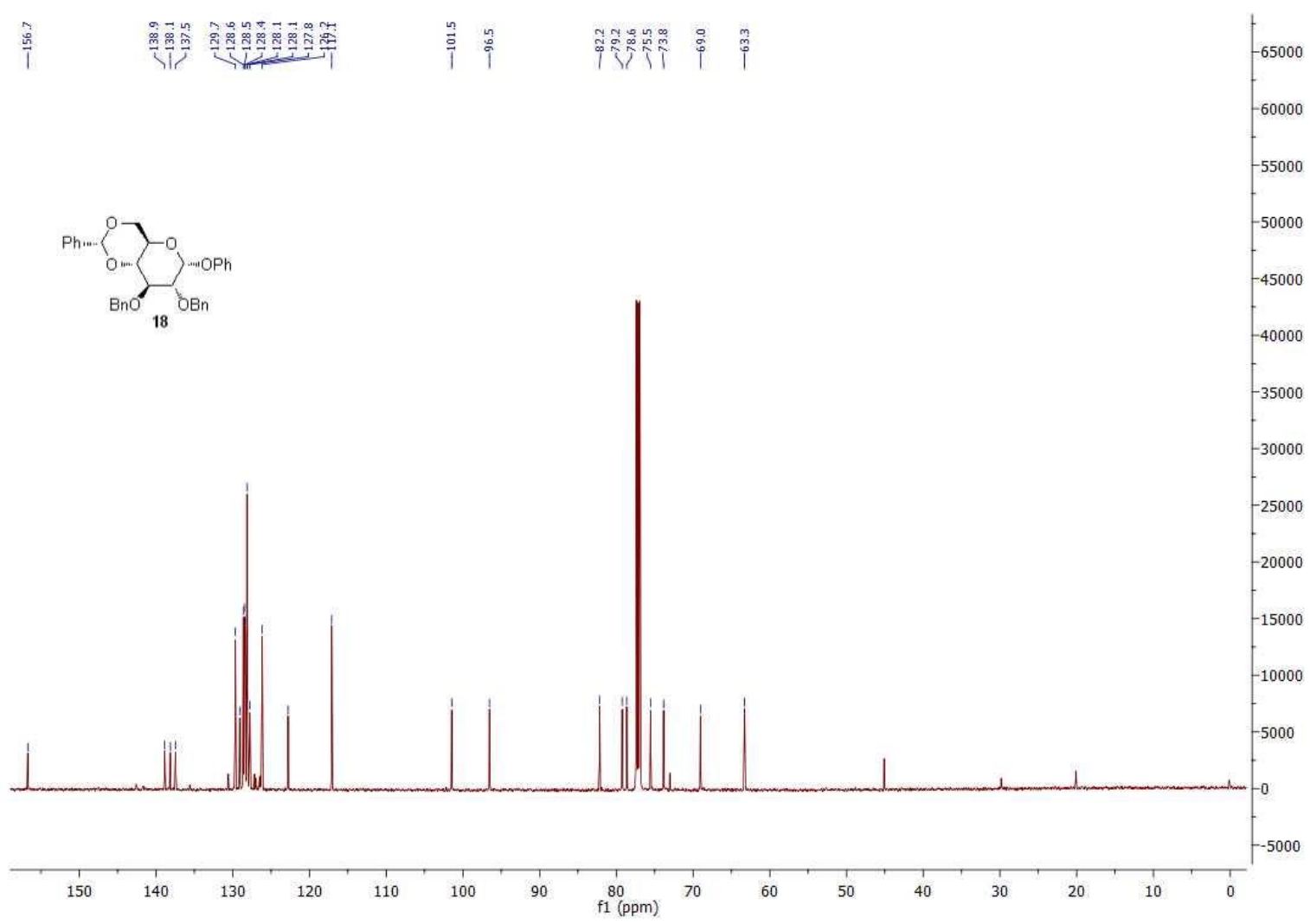

${ }^{13} \mathrm{C}-\mathrm{NMR}$ Spectrum of Compound $\mathbf{1 8}\left(\mathrm{CDCl}_{3}\right)$ 


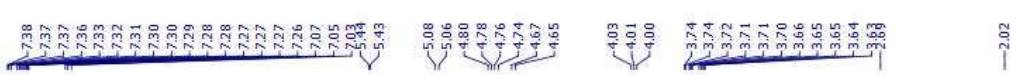

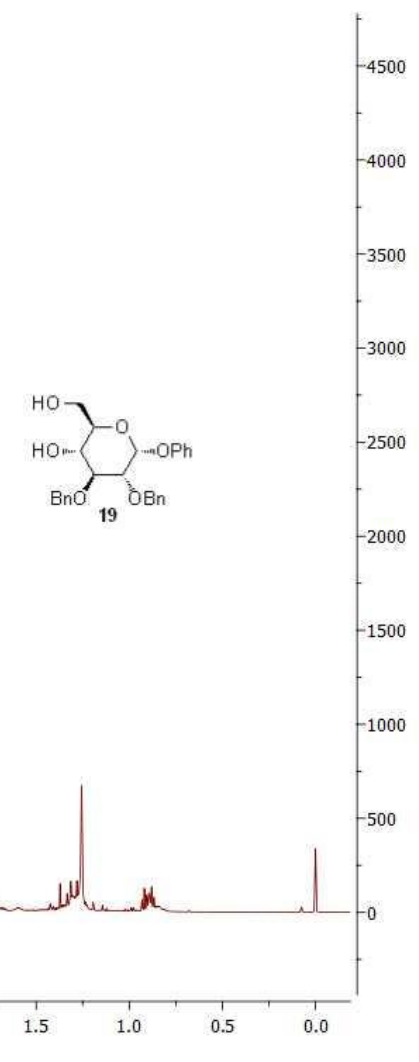

${ }^{1} \mathrm{H}-\mathrm{NMR}$ Spectrum of Compound $19\left(\mathrm{CDCl}_{3}\right)$

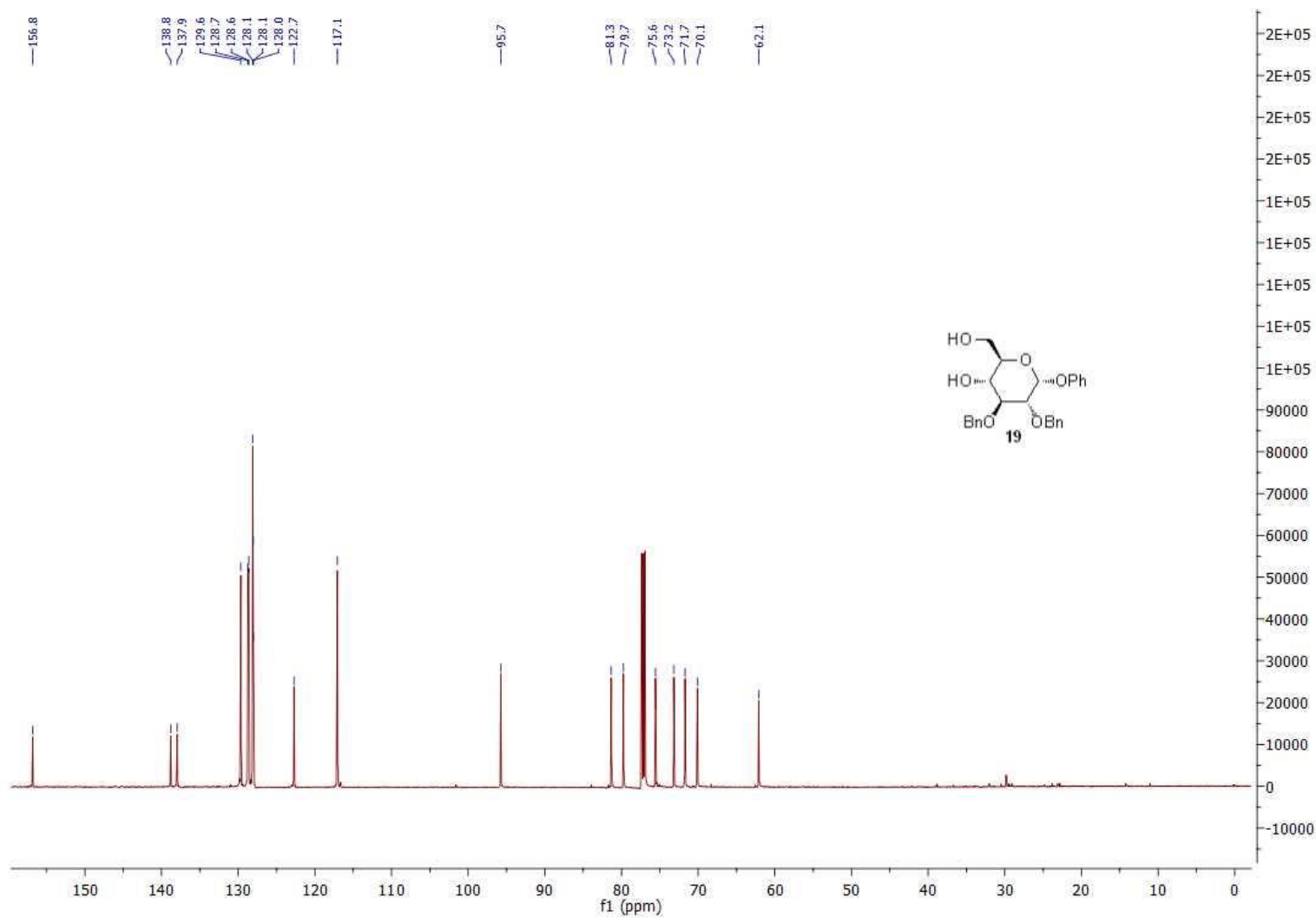

${ }^{13} \mathrm{C}-\mathrm{NMR}$ Spectrum of Compound $\mathbf{1 9}\left(\mathrm{CDCl}_{3}\right)$ 


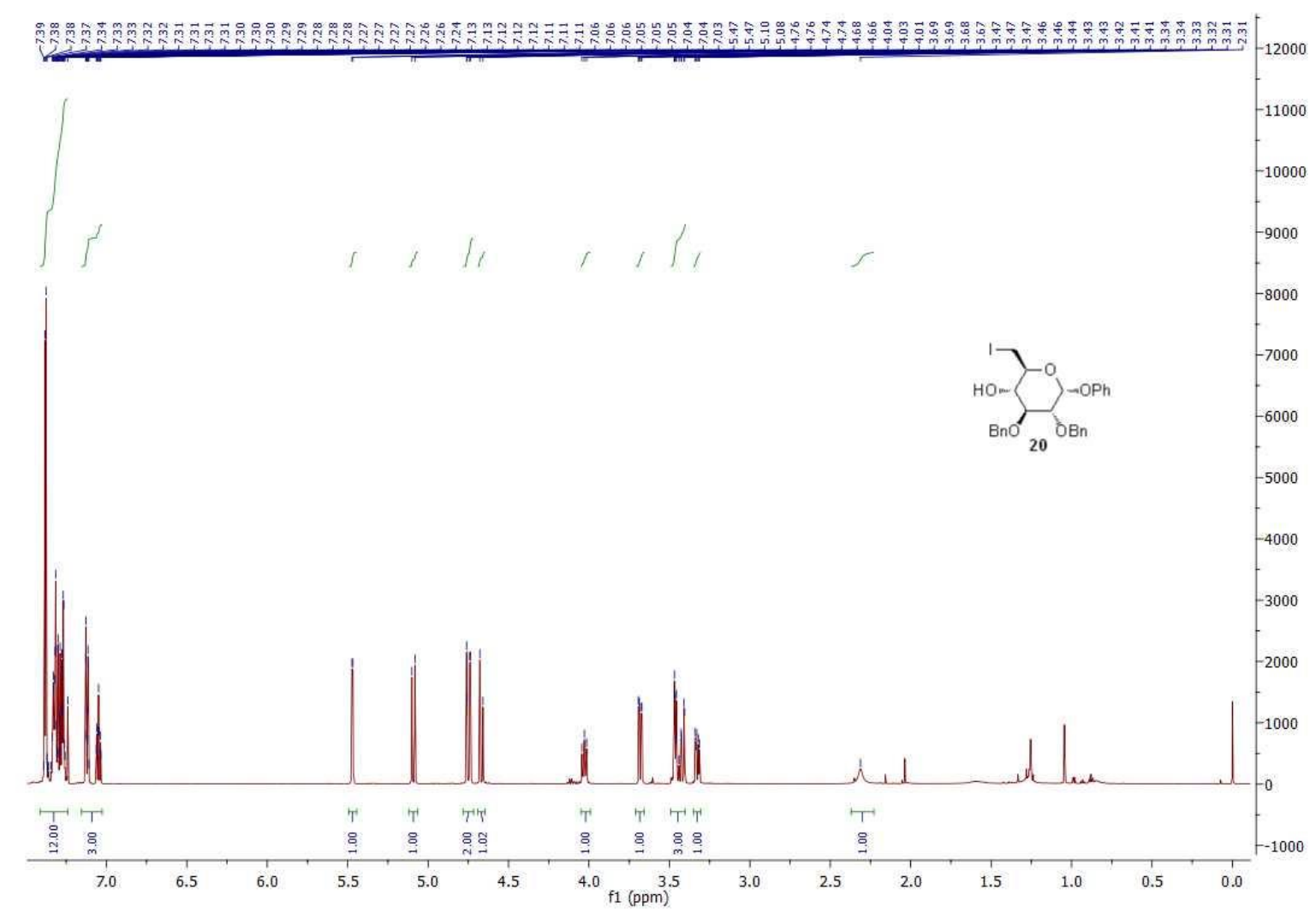

${ }^{1} \mathrm{H}-\mathrm{NMR}$ Spectrum of Compound $20\left(\mathrm{CDCl}_{3}\right)$

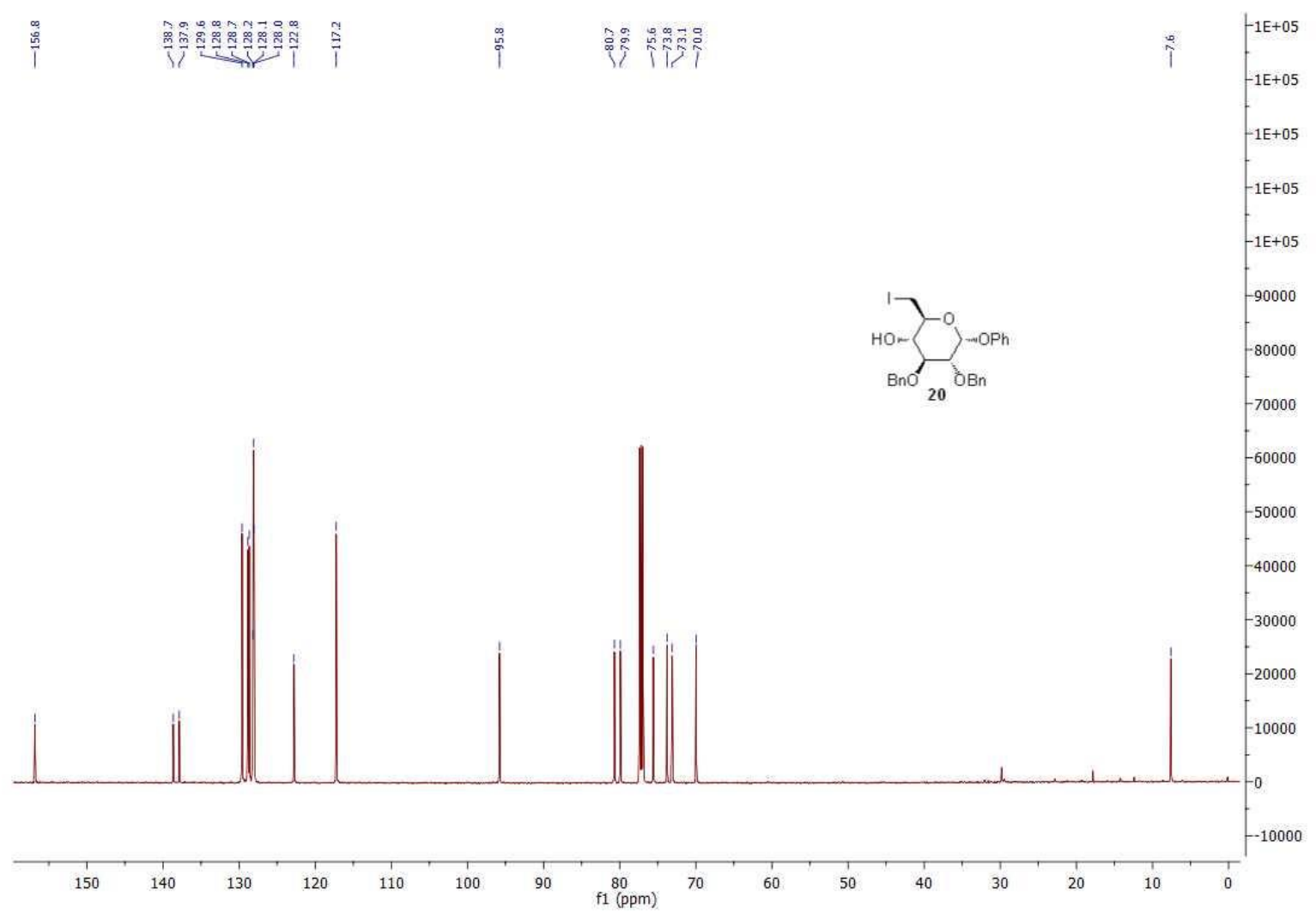

${ }^{13} \mathrm{C}-\mathrm{NMR}$ Spectrum of Compound $20\left(\mathrm{CDCl}_{3}\right)$ 


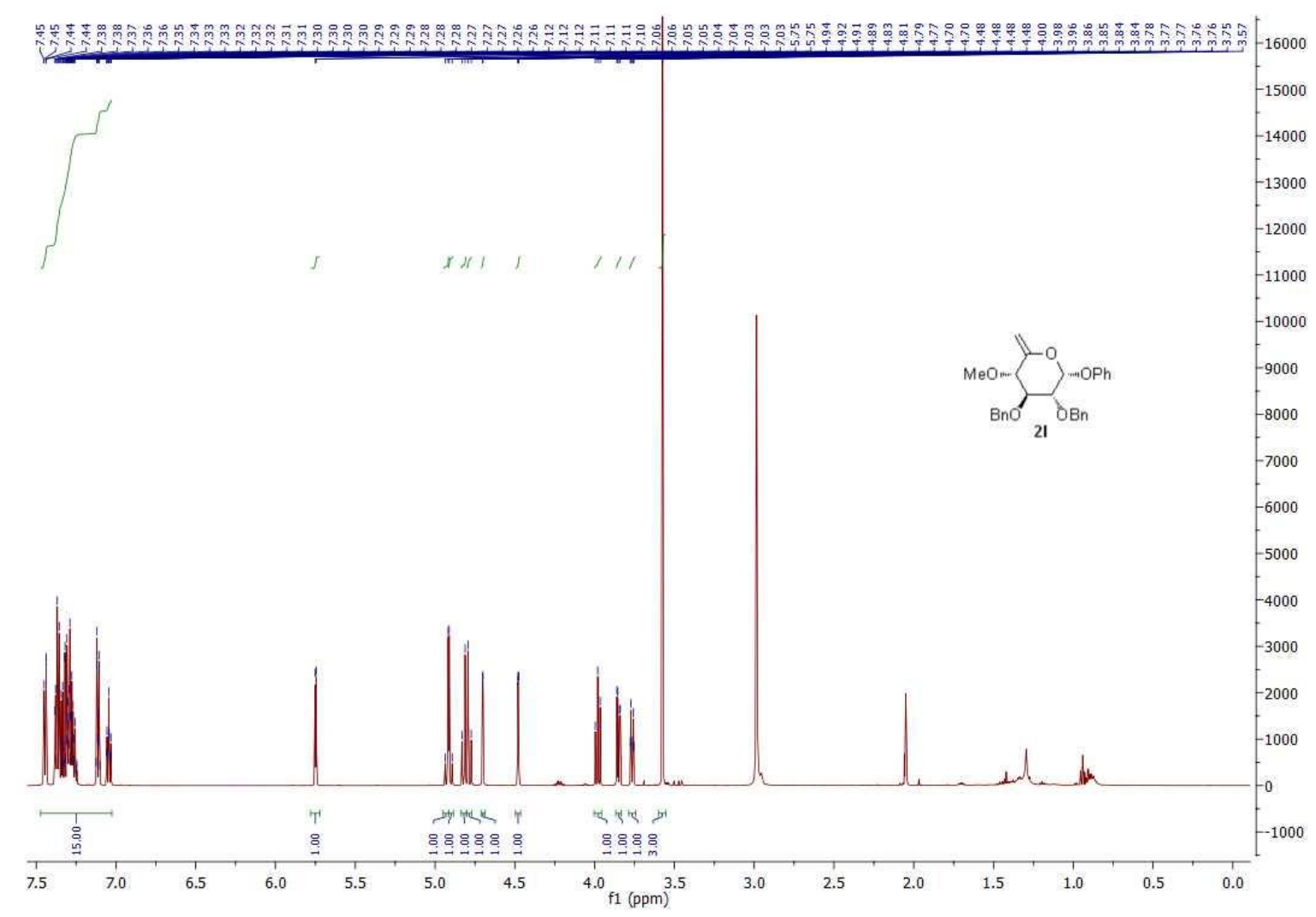

${ }^{1}$ H-NMR Spectrum of Compound 21 (Acetone-d6)

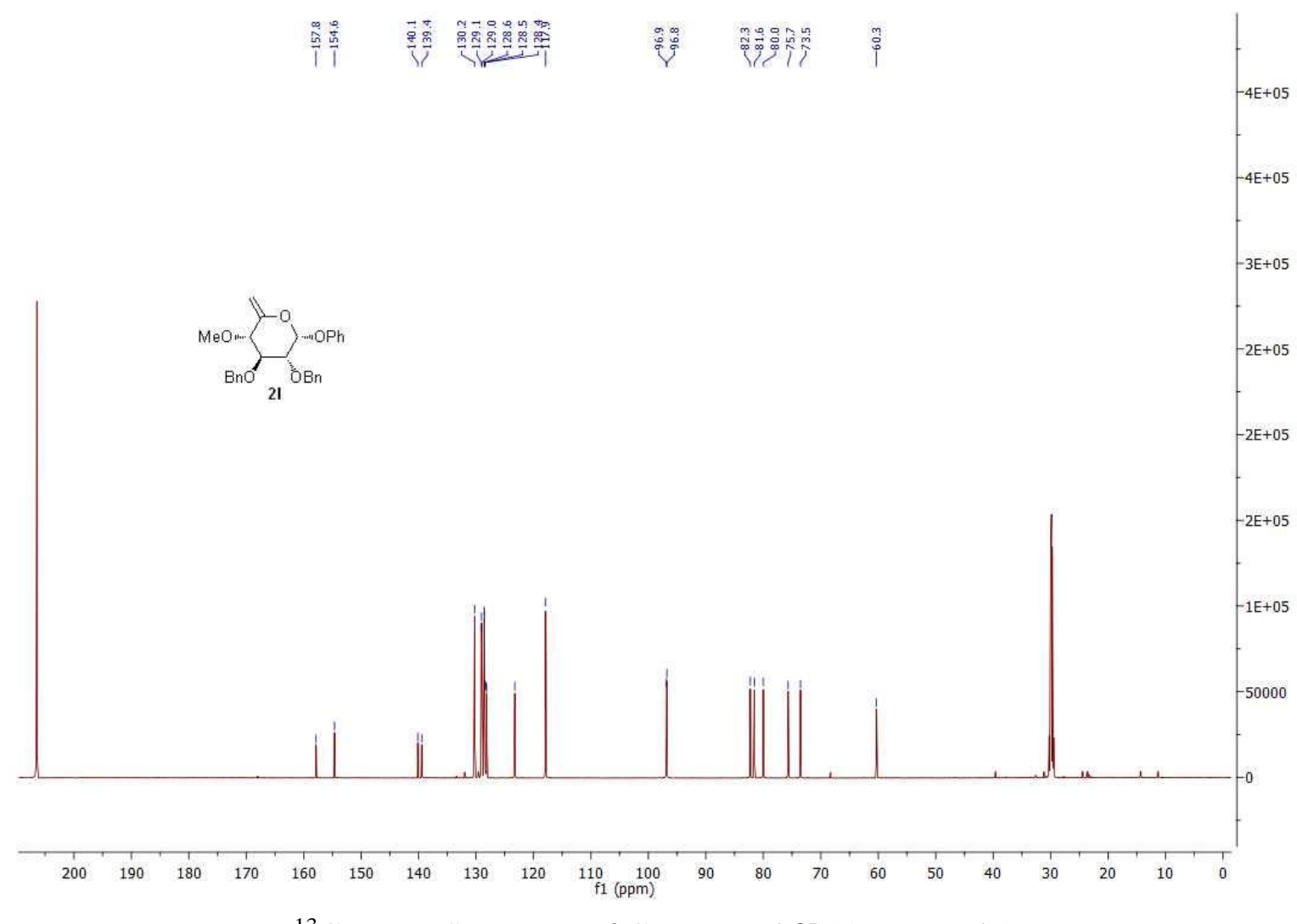

${ }^{13} \mathrm{C}-\mathrm{NMR}$ Spectrum of Compound 2l (Acetone-d6) 


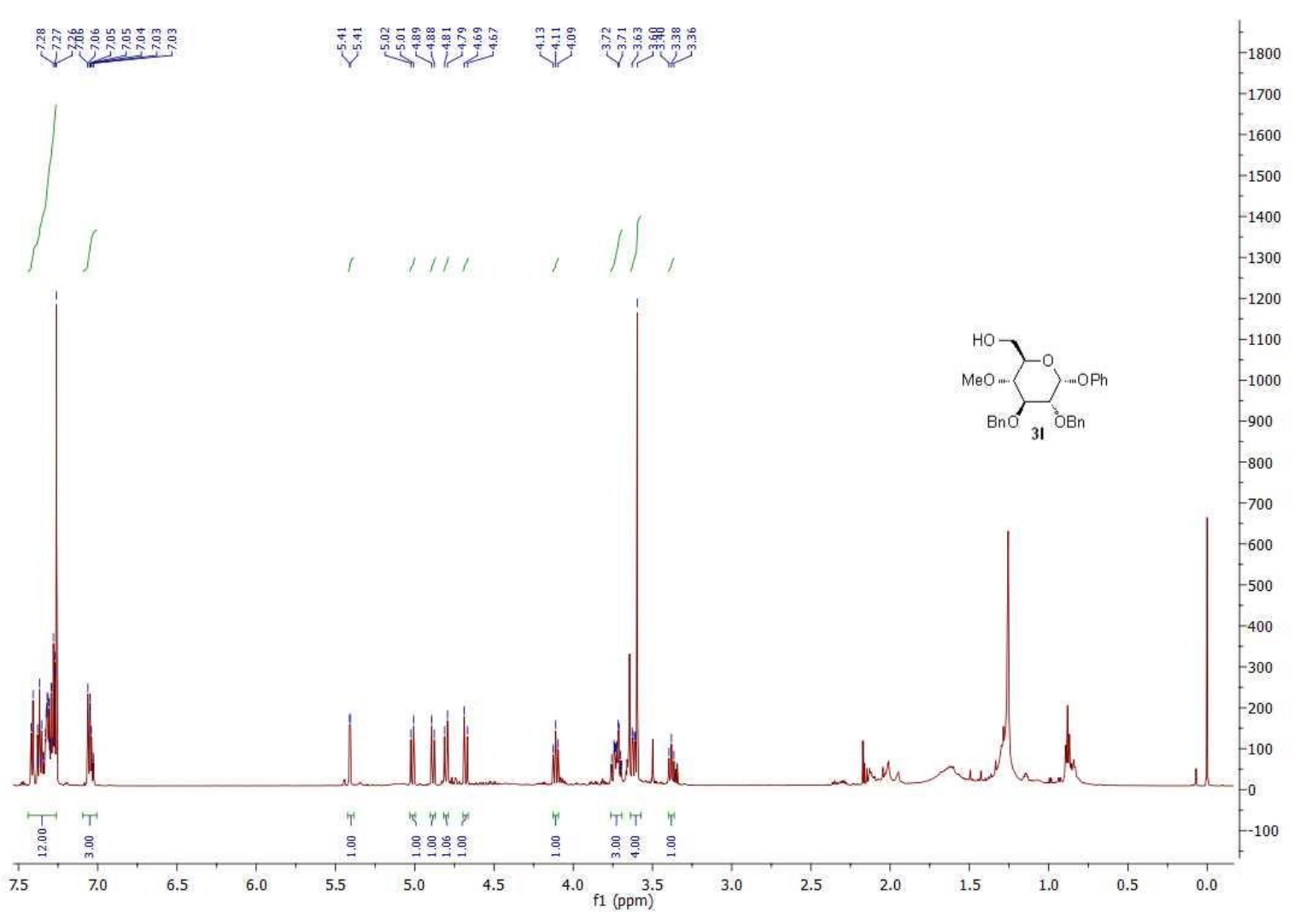

${ }^{1} \mathrm{H}-\mathrm{NMR}$ Spectrum of Compound $\mathbf{3 l}\left(\mathrm{CDCl}_{3}\right)$

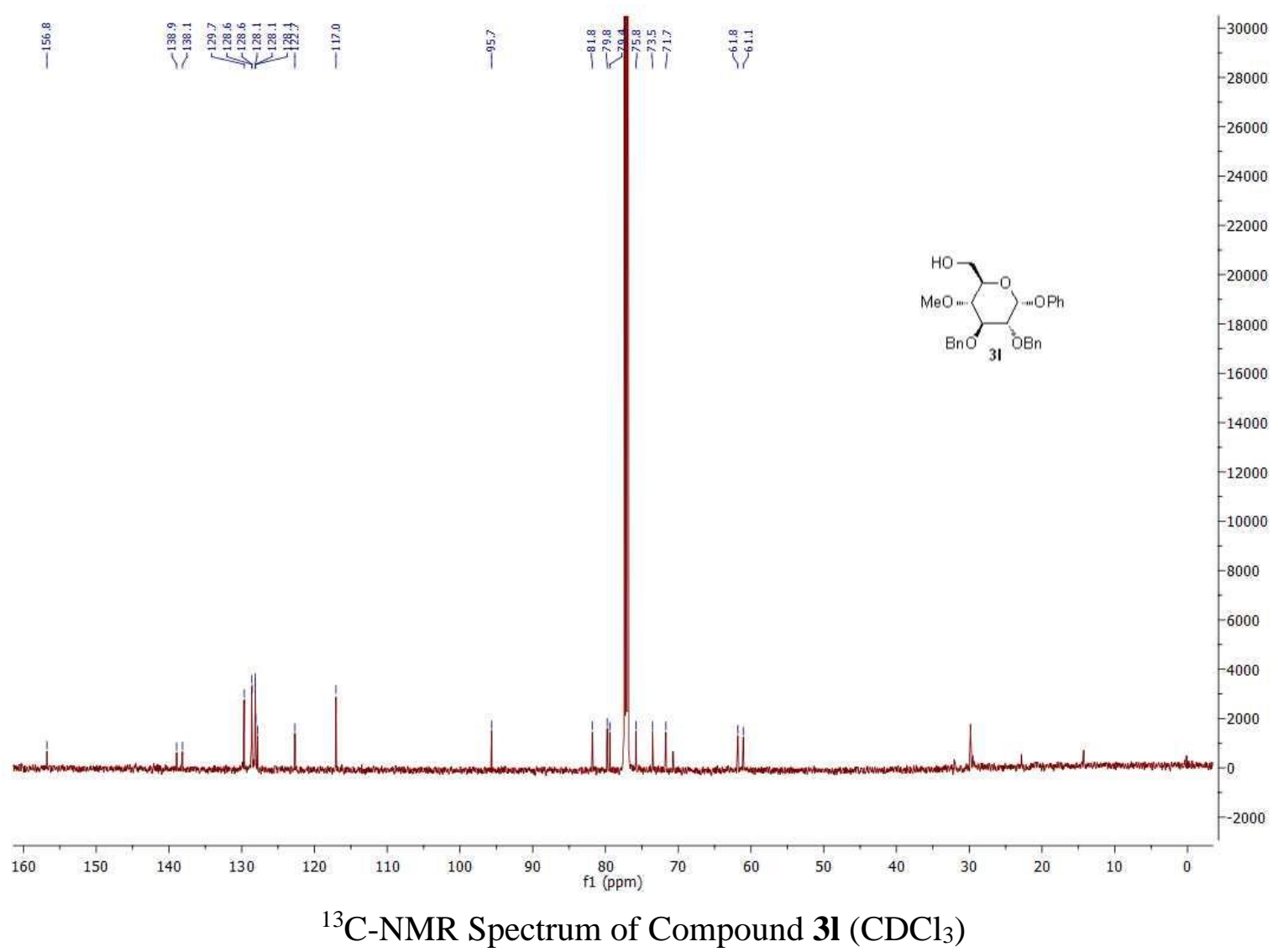




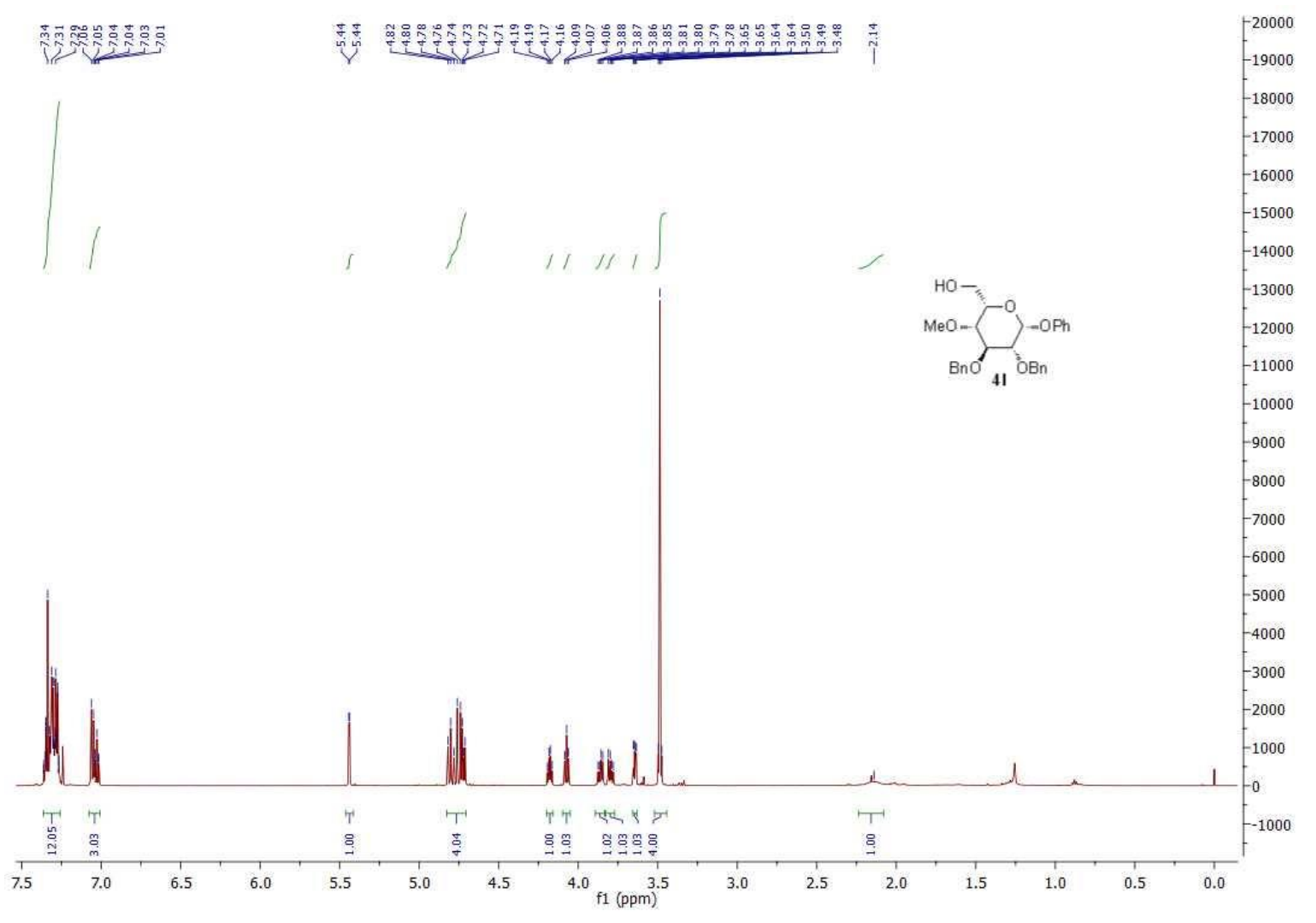

${ }^{1} \mathrm{H}-\mathrm{NMR}$ Spectrum of Compound $4 \mathbf{l}\left(\mathrm{CDCl}_{3}\right)$

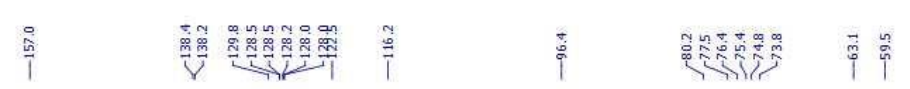

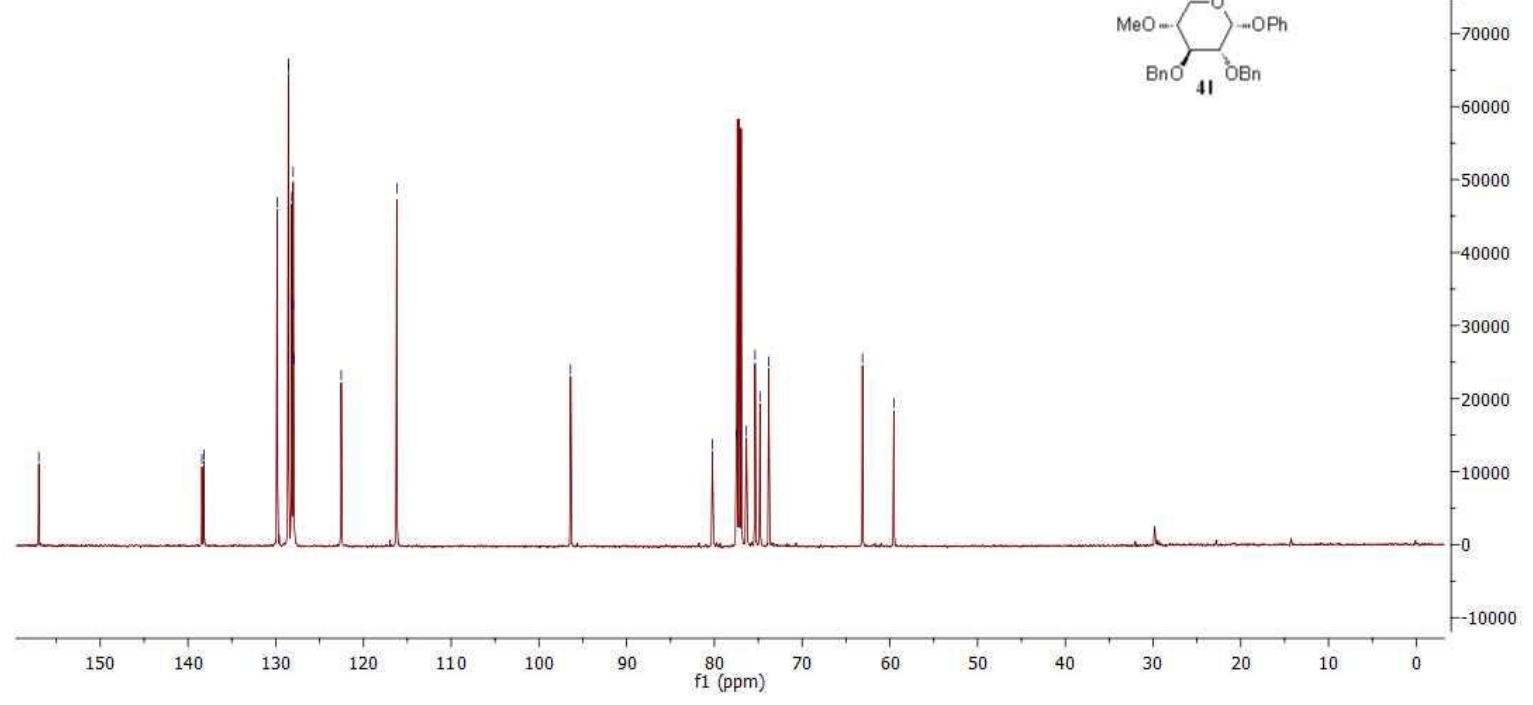

${ }^{13} \mathrm{C}-\mathrm{NMR}$ Spectrum of Compound $4 \mathbf{l}\left(\mathrm{CDCl}_{3}\right)$ 


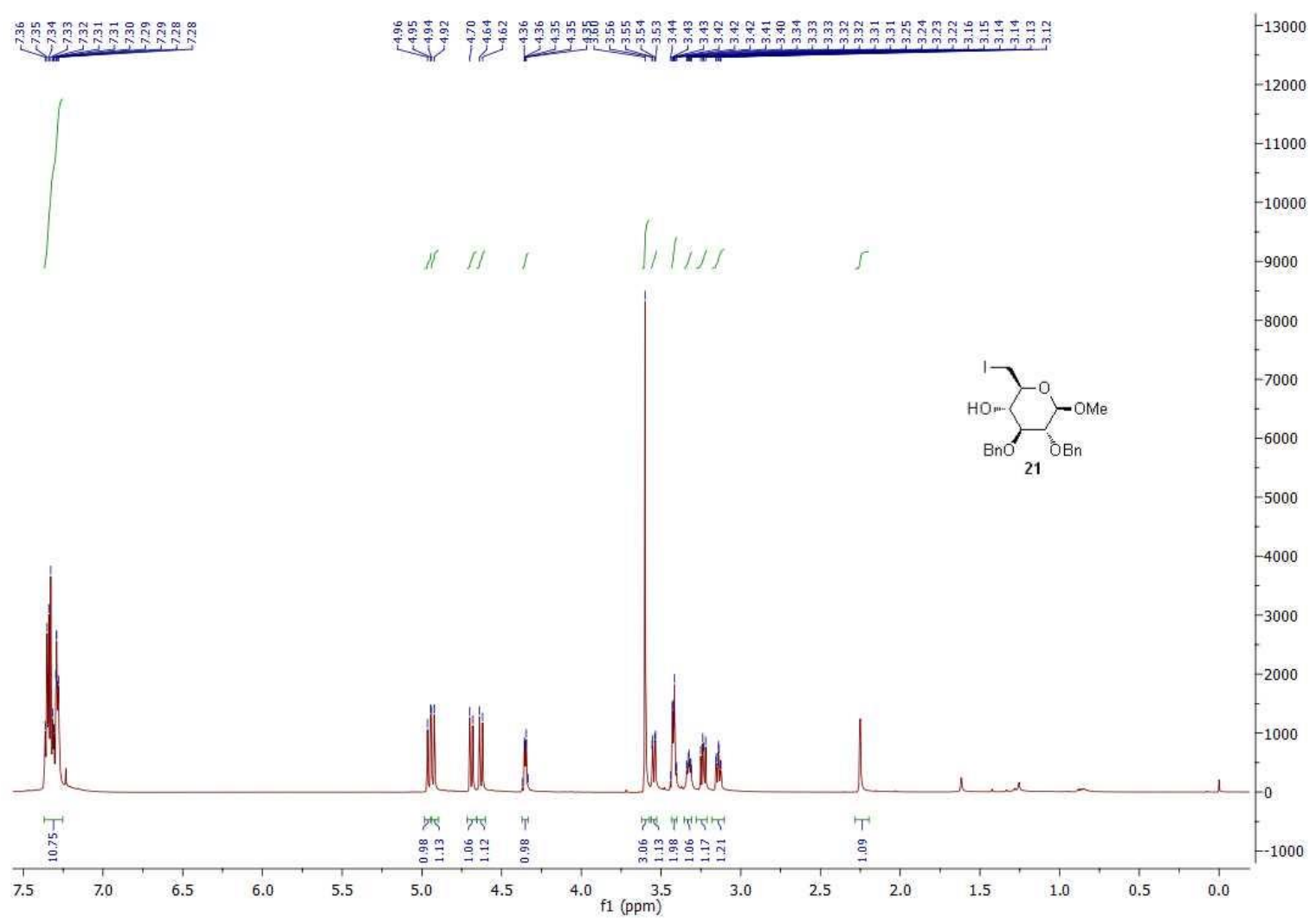

${ }^{1} \mathrm{H}-\mathrm{NMR}$ Spectrum of Compound $21\left(\mathrm{CDCl}_{3}\right)$

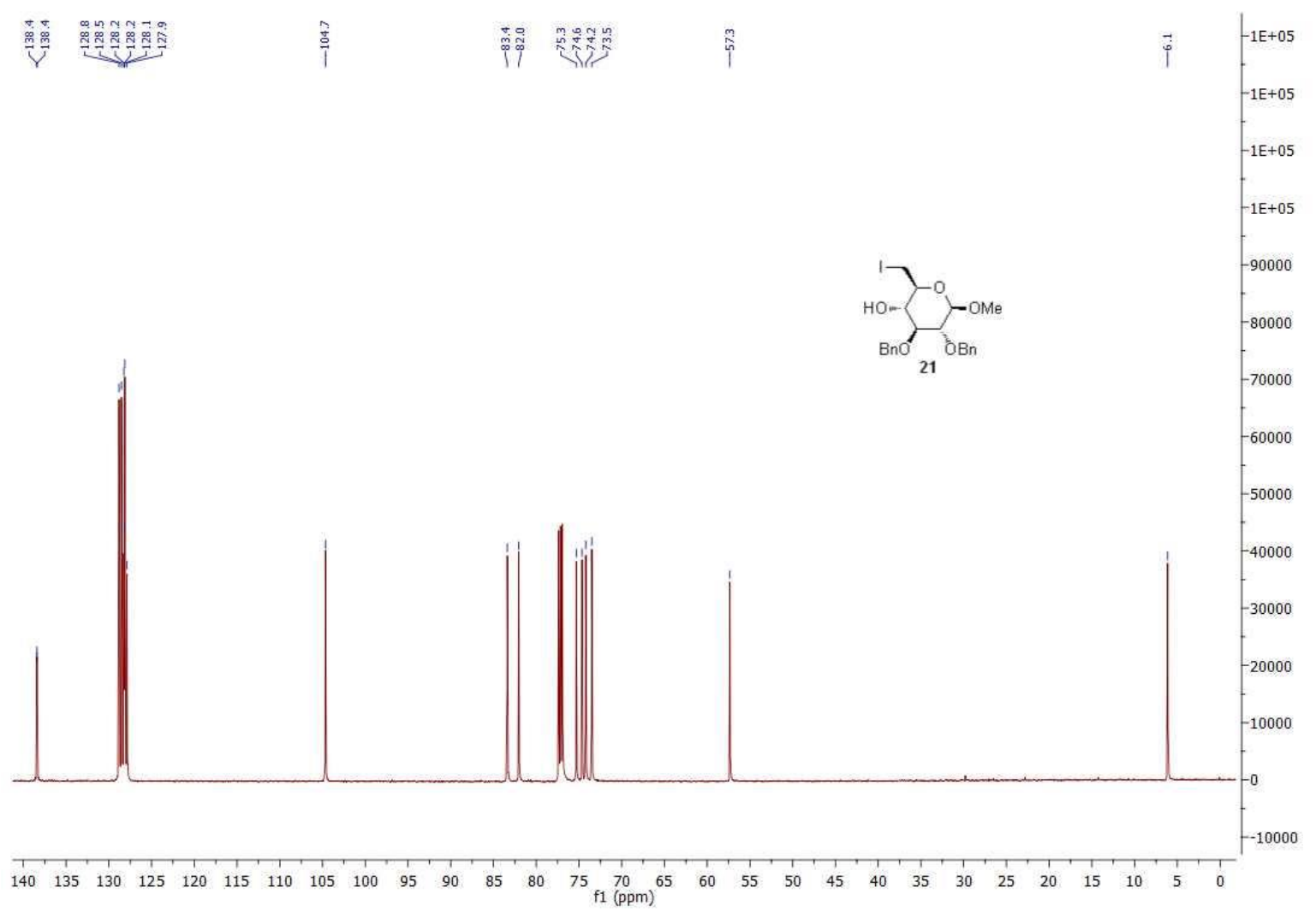

${ }^{13} \mathrm{C}$-NMR Spectrum of Compound $21\left(\mathrm{CDCl}_{3}\right)$ 


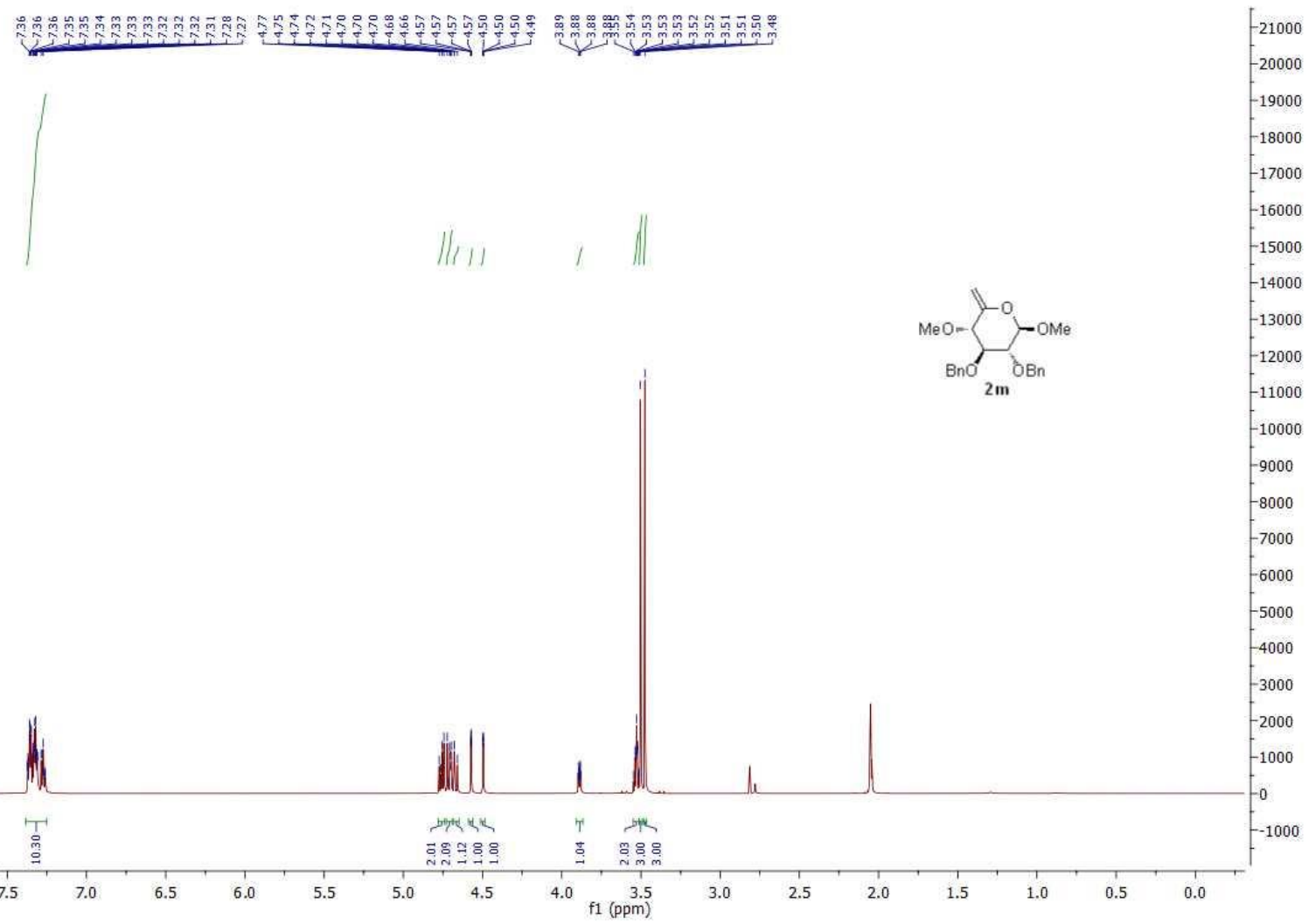

${ }^{1}$ H-NMR Spectrum of Compound $\mathbf{2 m}$ (Acetone-d6)

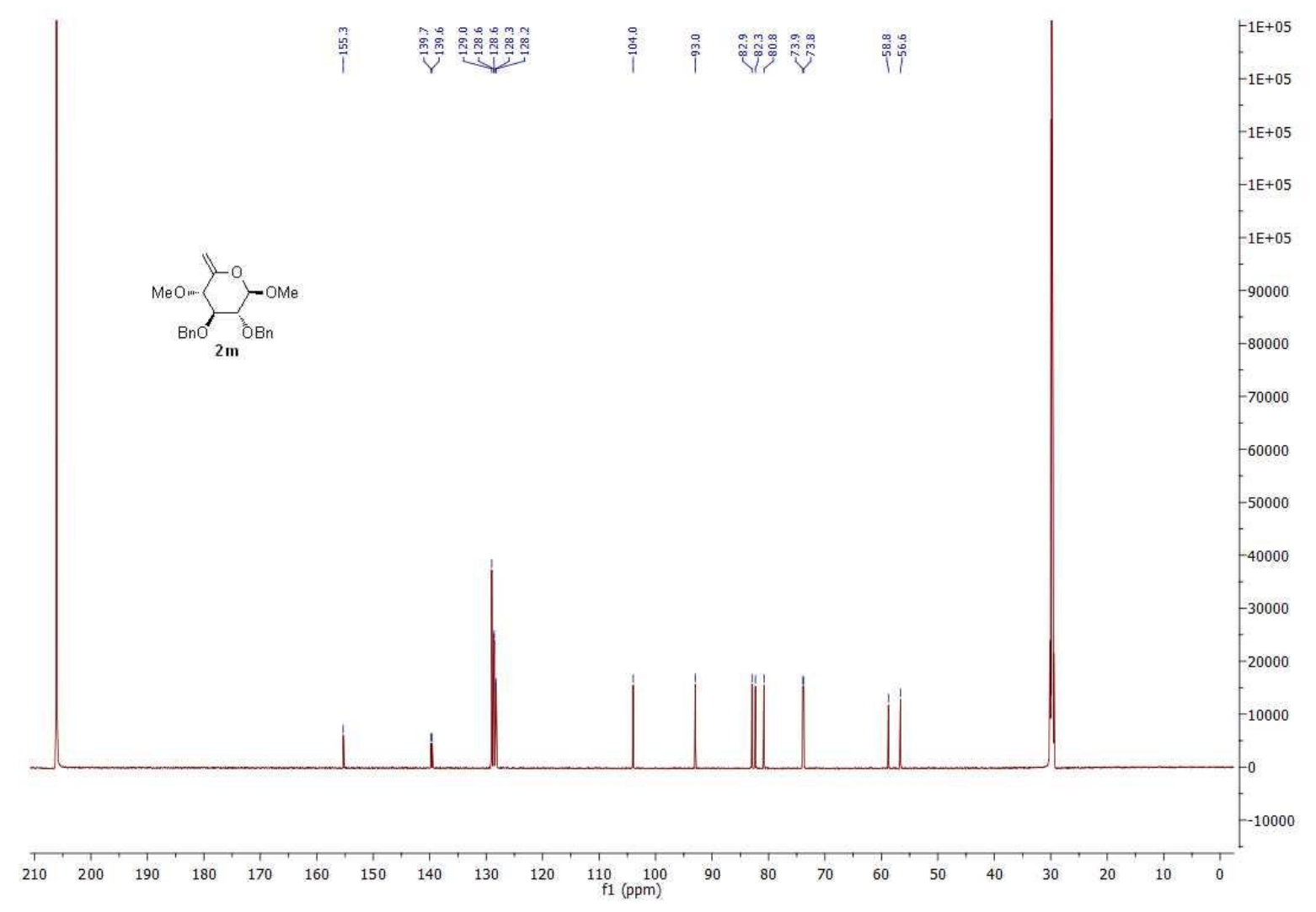

${ }^{13} \mathrm{C}-\mathrm{NMR}$ Spectrum of Compound $\mathbf{2 m}$ (Acetone-d6) 


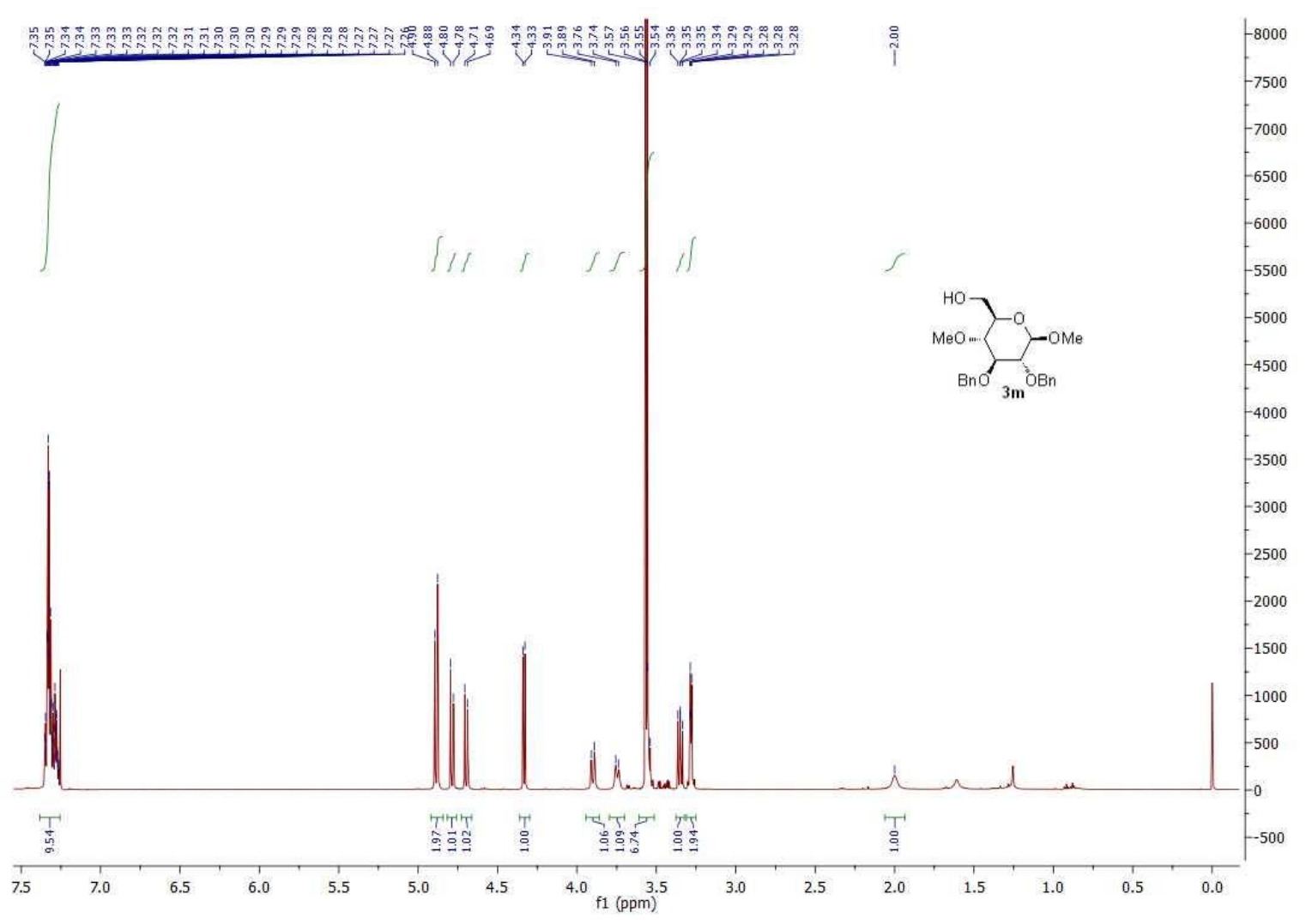

${ }^{1} \mathrm{H}-\mathrm{NMR}$ Spectrum of Compound $\mathbf{3 m}\left(\mathrm{CDCl}_{3}\right)$

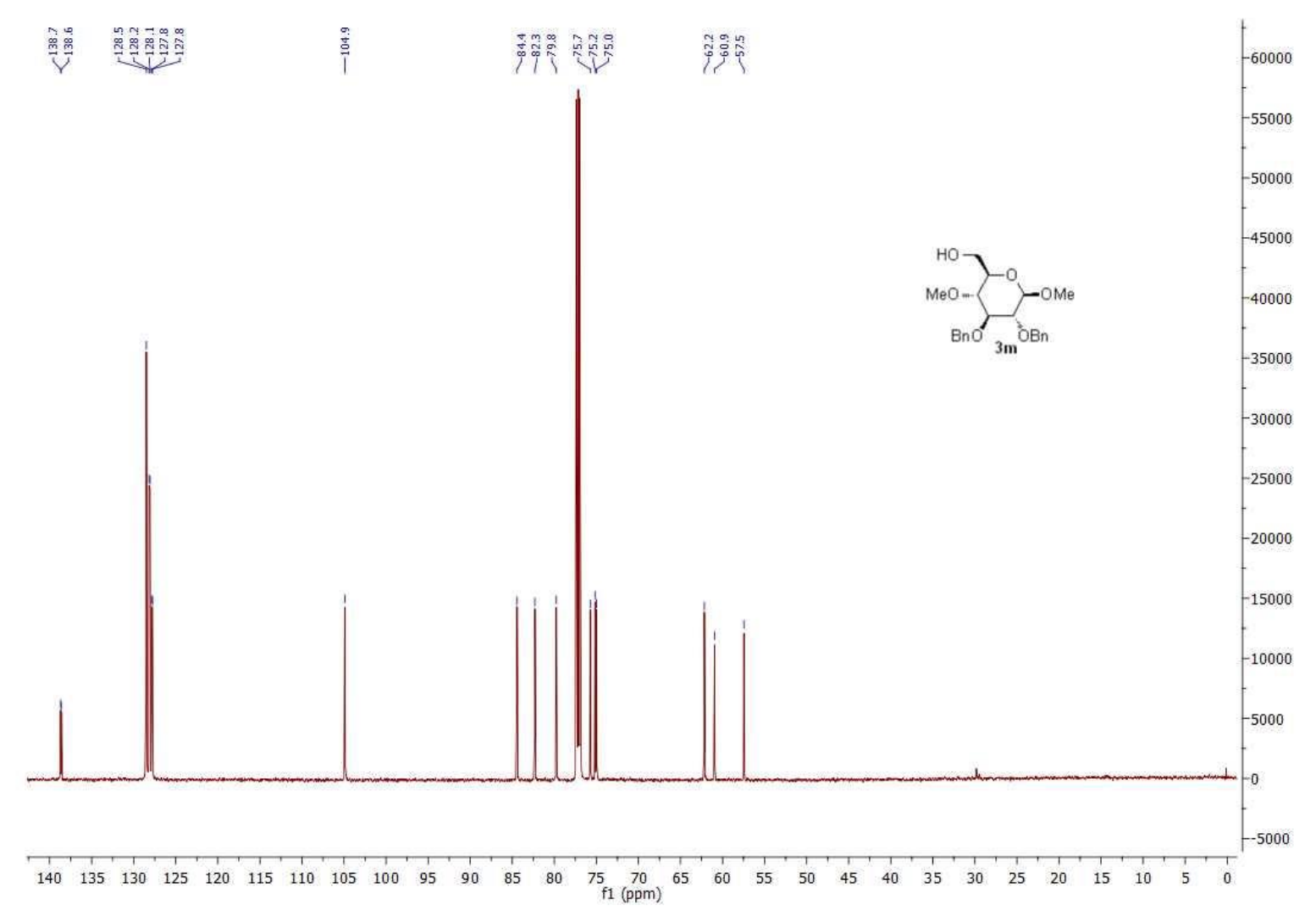

${ }^{13} \mathrm{C}-\mathrm{NMR}$ Spectrum of Compound $\mathbf{3 m}\left(\mathrm{CDCl}_{3}\right)$ 


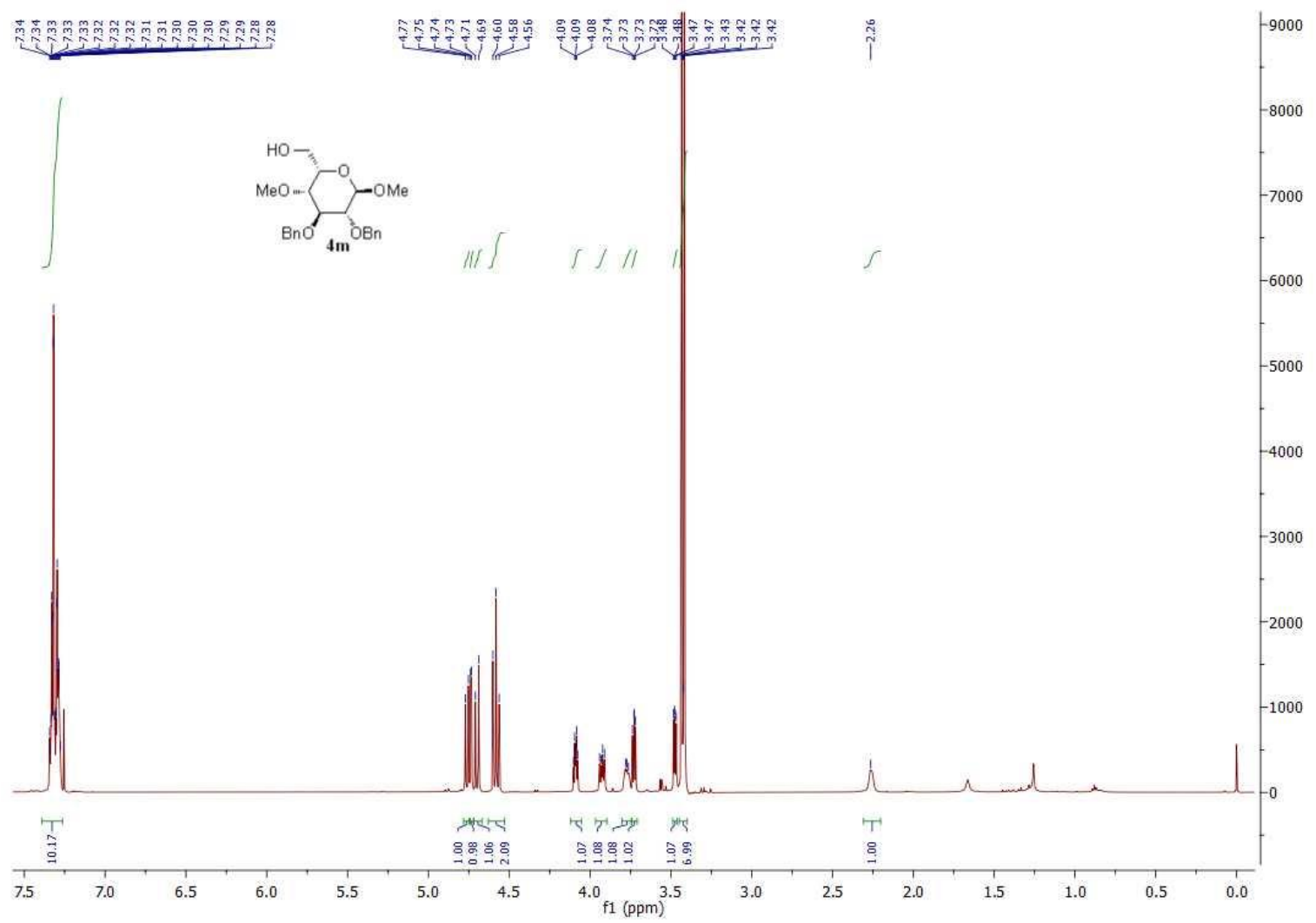

${ }^{1} \mathrm{H}-\mathrm{NMR}$ Spectrum of Compound $\mathbf{4 m}\left(\mathrm{CDCl}_{3}\right)$

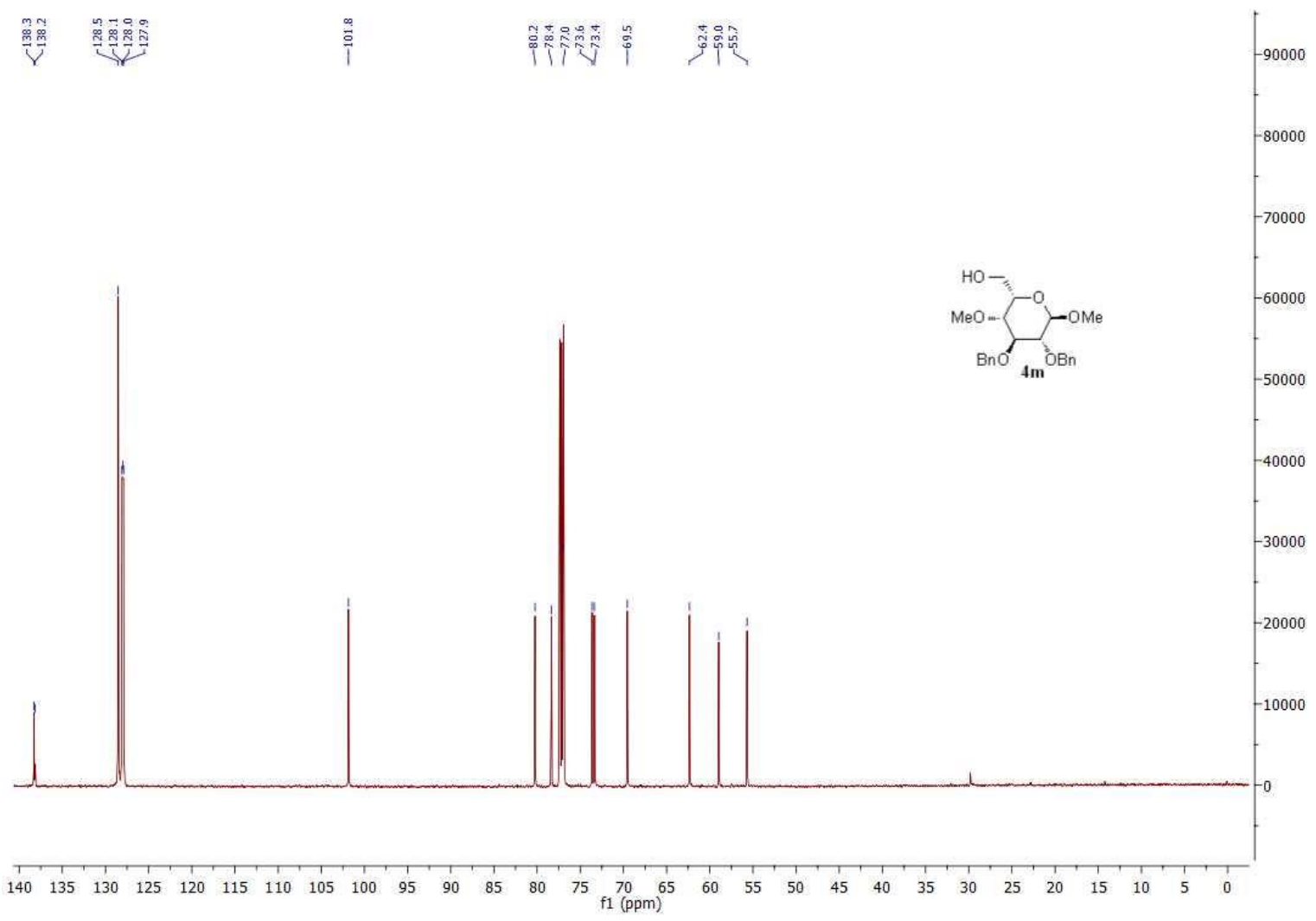

${ }^{13} \mathrm{C}$-NMR Spectrum of Compound $\mathbf{4 m}\left(\mathrm{CDCl}_{3}\right)$ 


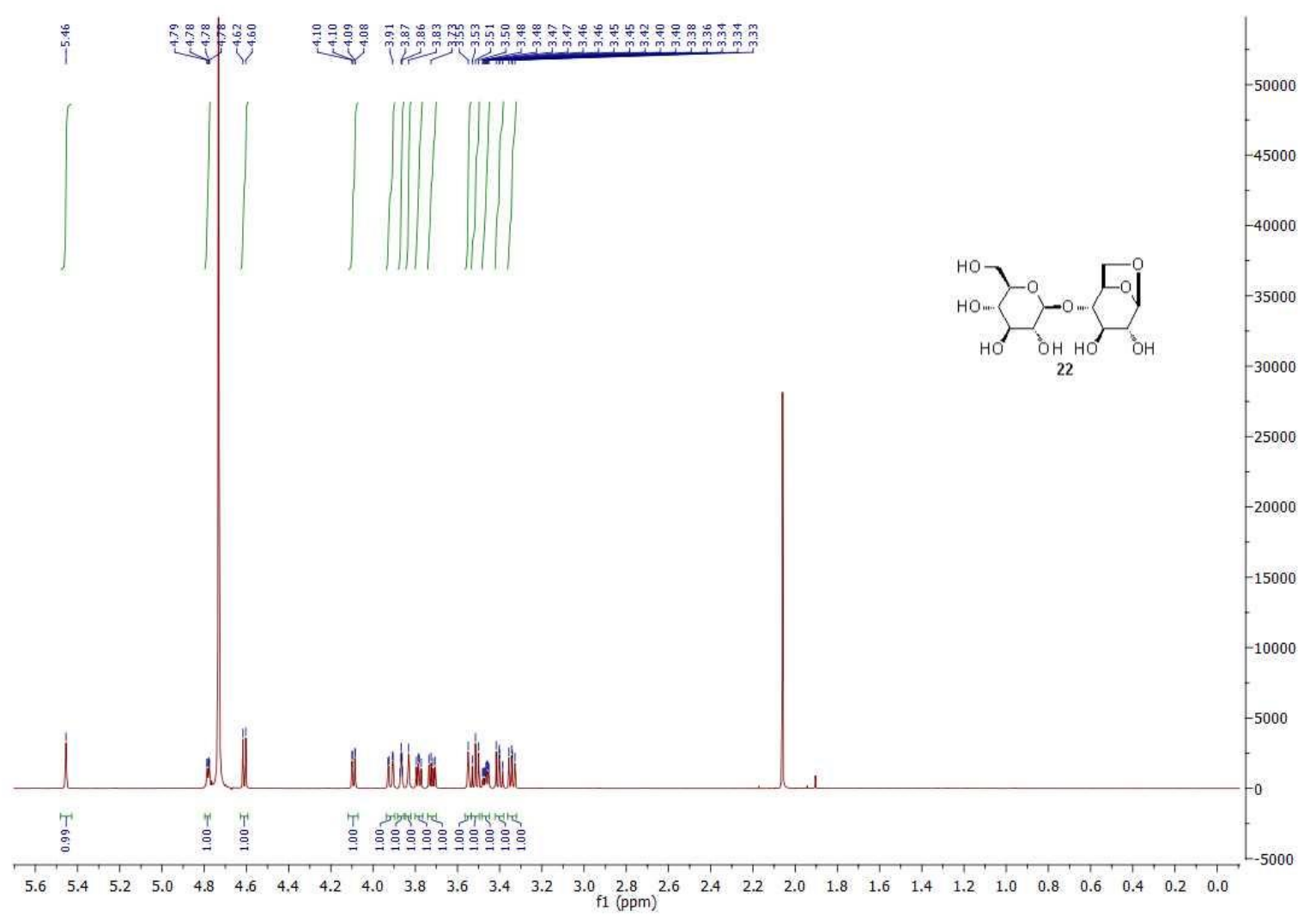

${ }^{1} \mathrm{H}-\mathrm{NMR}$ Spectrum of Compound $22\left(\mathrm{D}_{2} \mathrm{O}\right)$

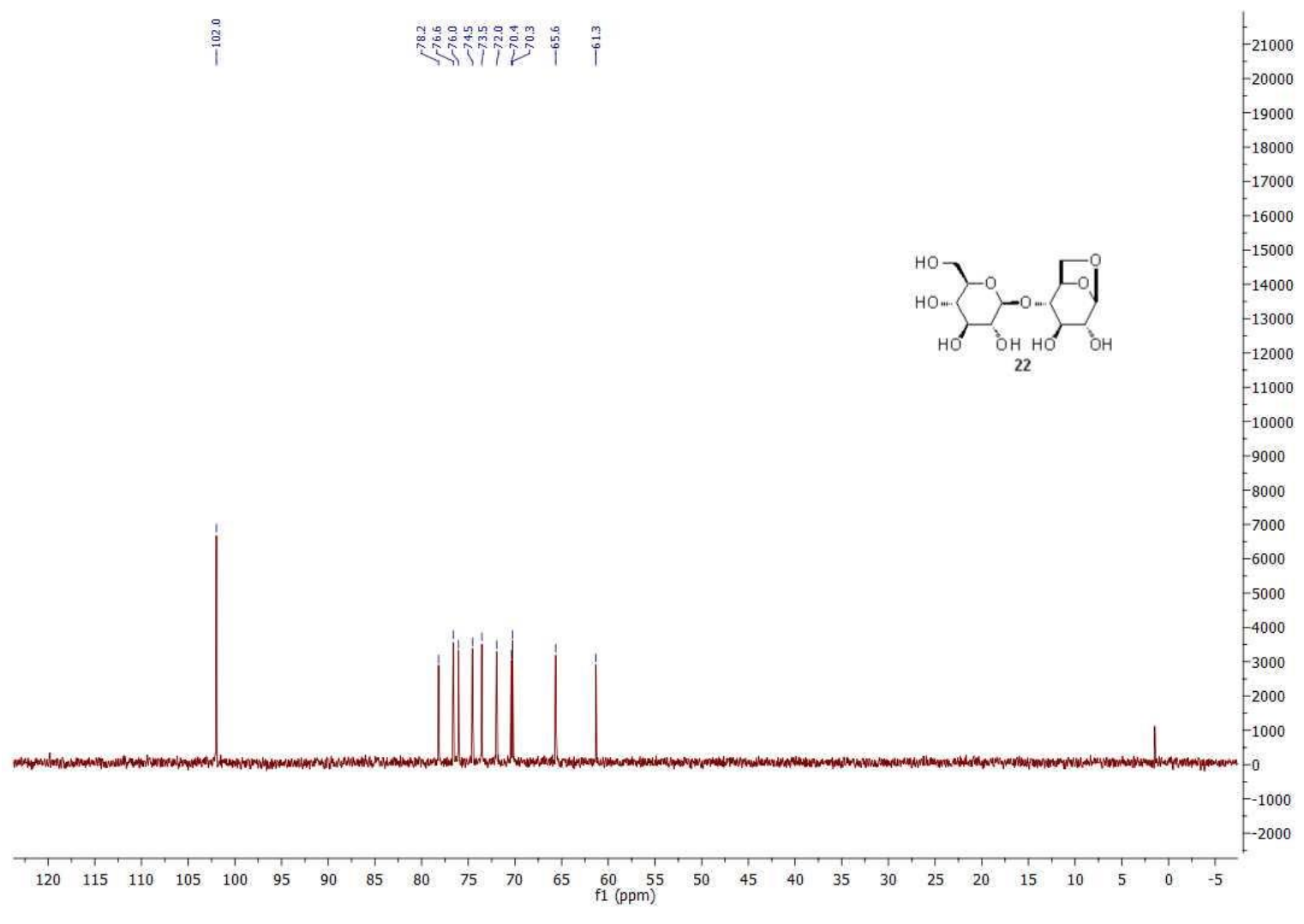

${ }^{13} \mathrm{C}-\mathrm{NMR}$ Spectrum of Compound $22\left(\mathrm{D}_{2} \mathrm{O}\right)$ 


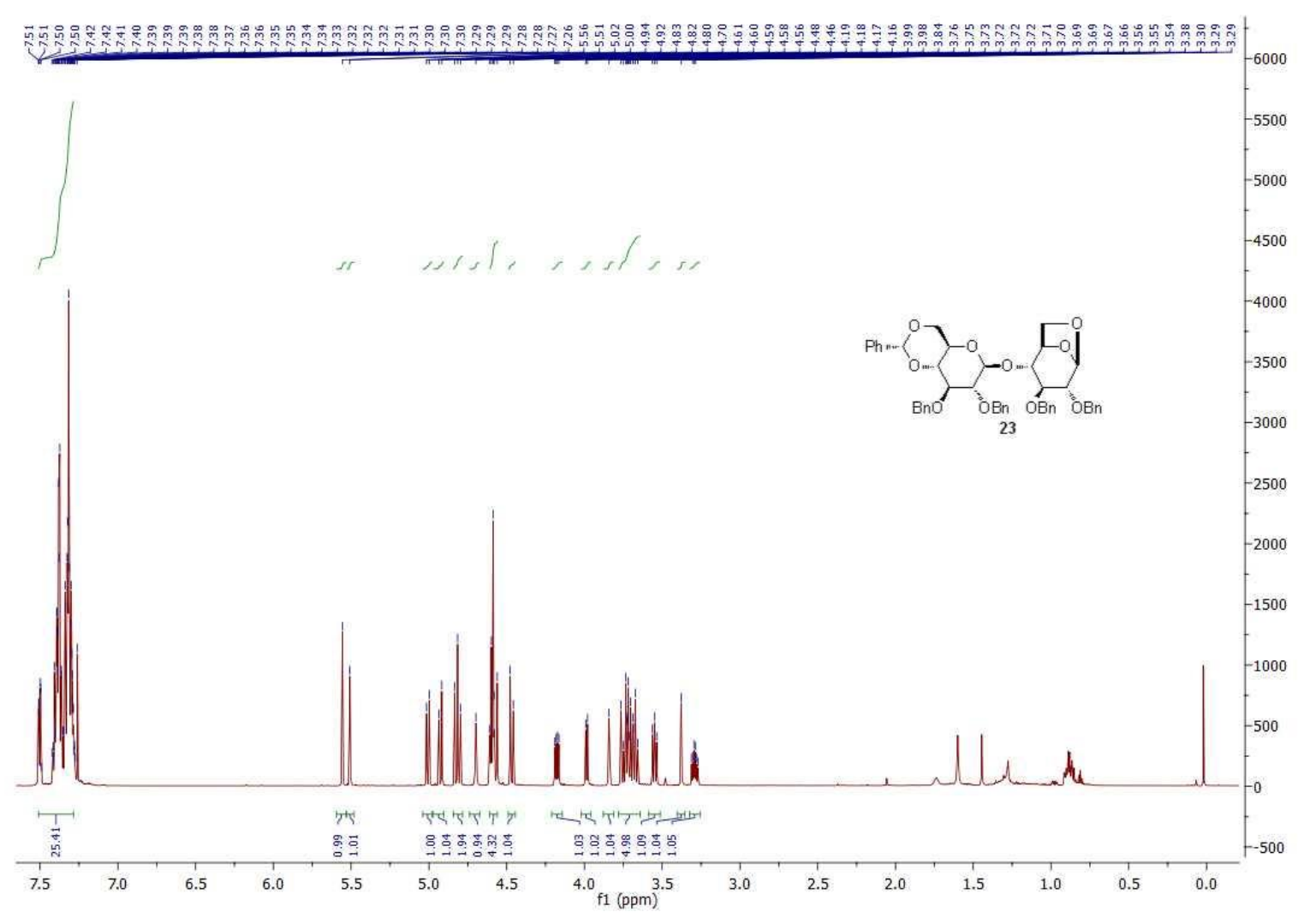

${ }^{1} \mathrm{H}-\mathrm{NMR}$ Spectrum of Compound $23\left(\mathrm{CDCl}_{3}\right)$

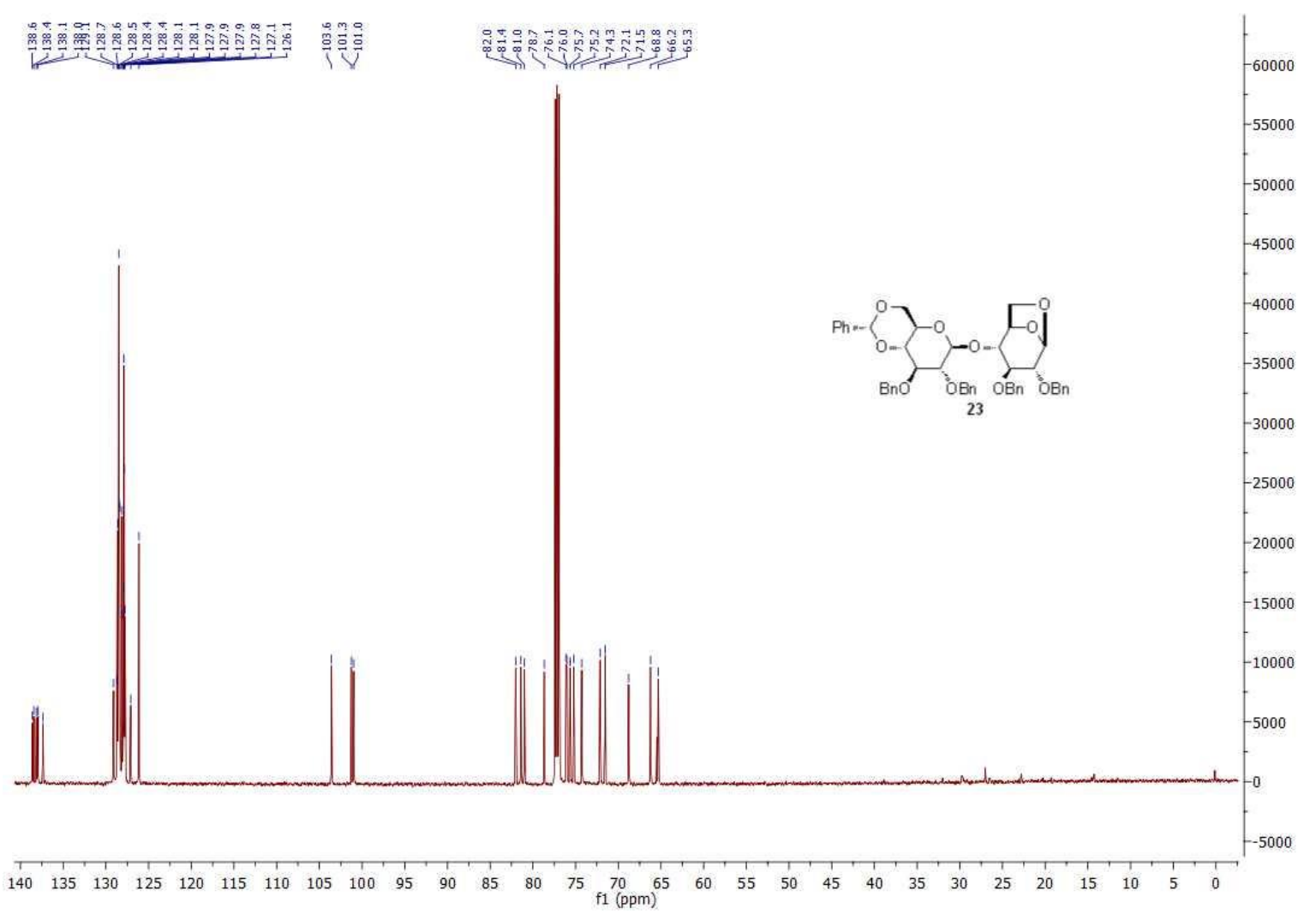

${ }^{13} \mathrm{C}-\mathrm{NMR}$ Spectrum of Compound $23\left(\mathrm{CDCl}_{3}\right)$ 


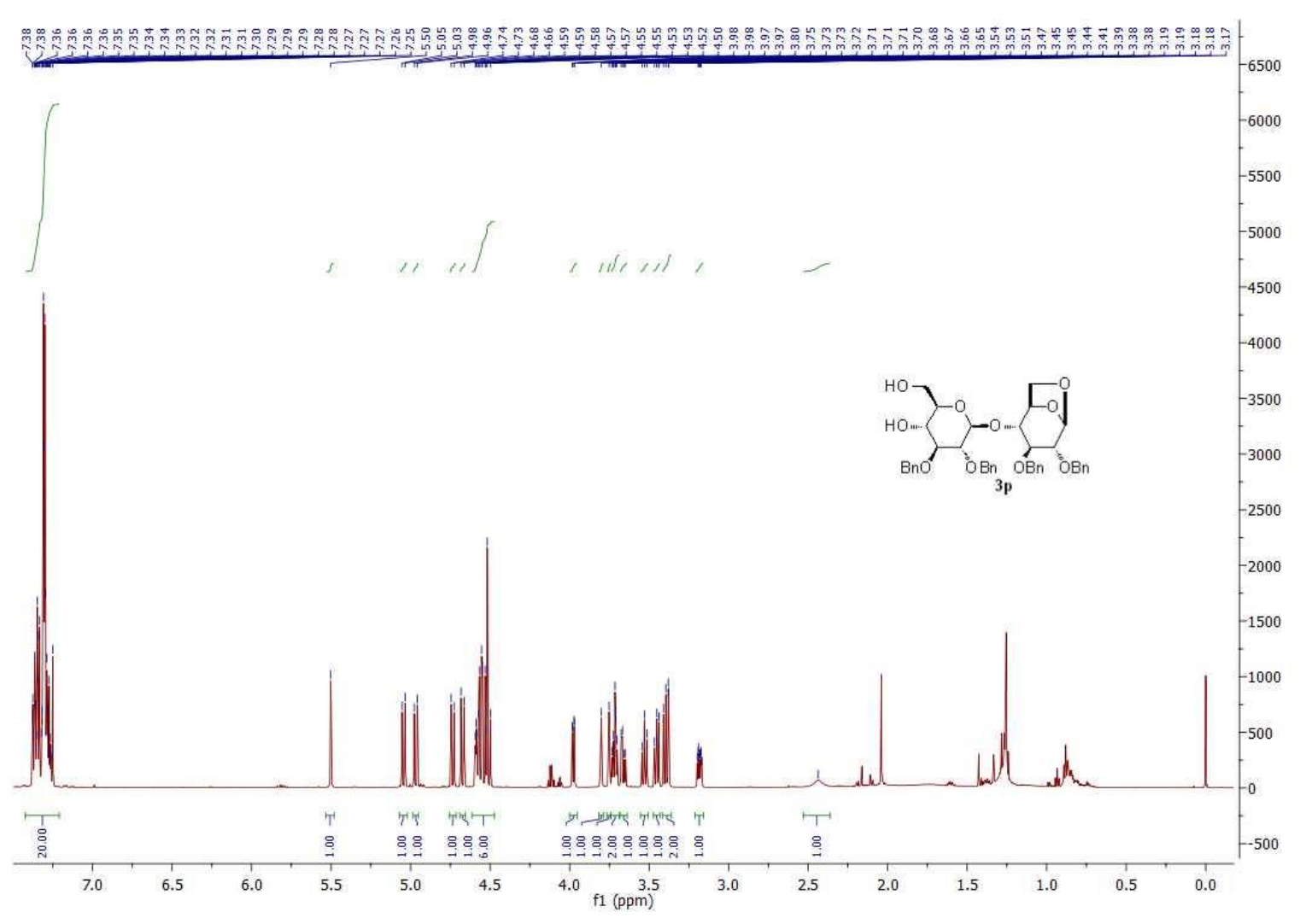

${ }^{1} \mathrm{H}-\mathrm{NMR}$ Spectrum of Compound $\mathbf{3 p}\left(\mathrm{CDCl}_{3}\right)$

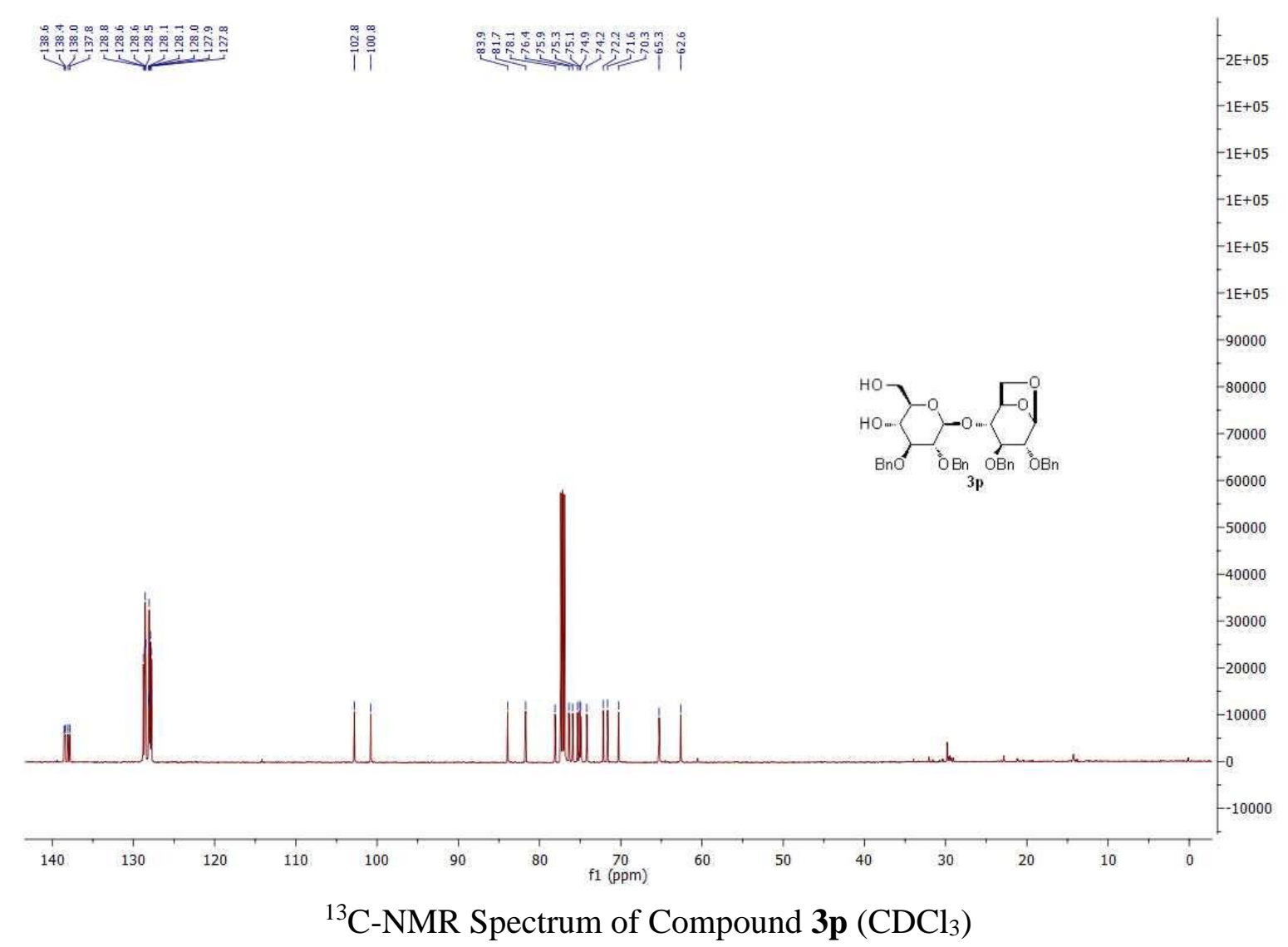




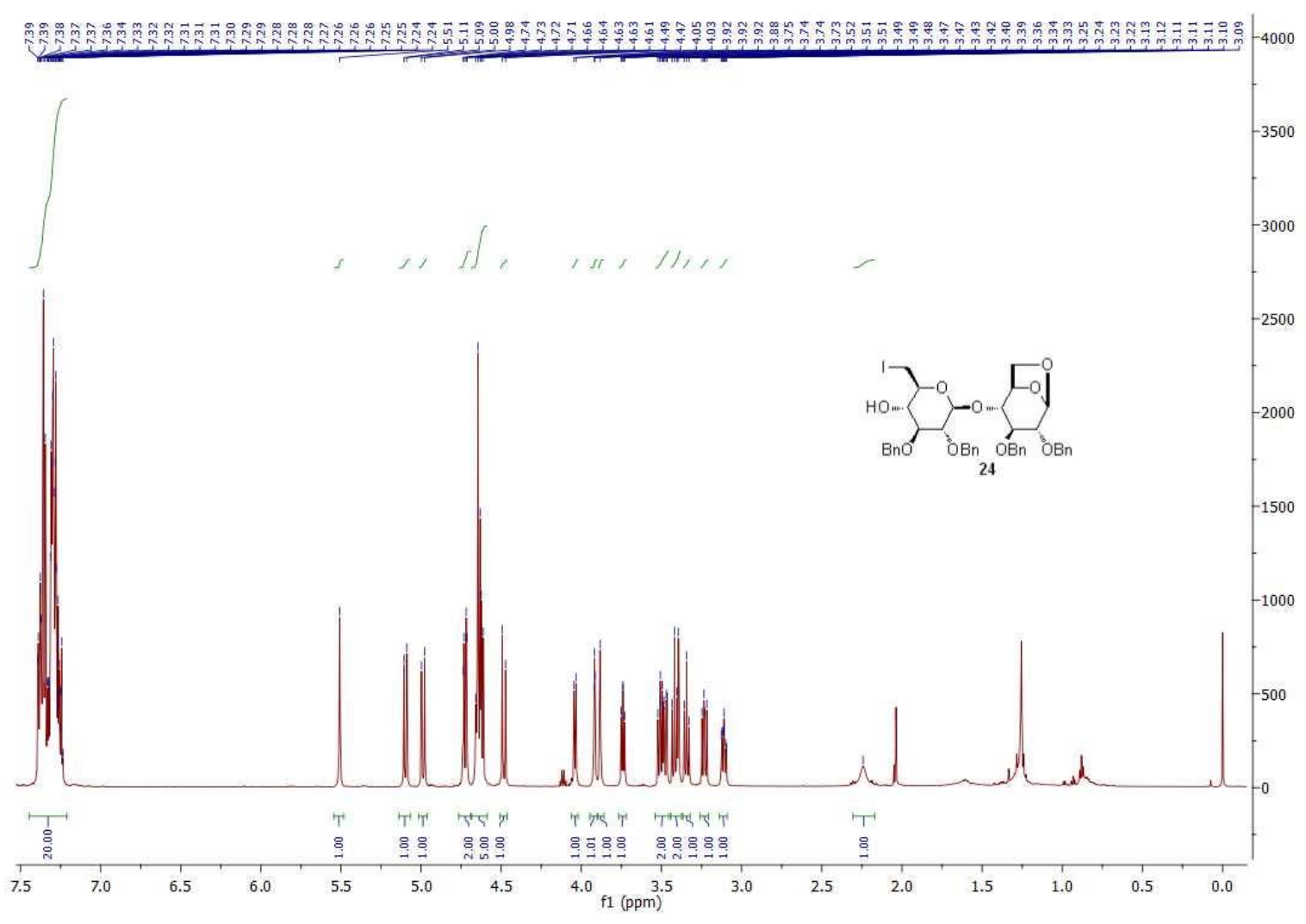

${ }^{1} \mathrm{H}-\mathrm{NMR}$ Spectrum of Compound $\mathbf{2 4}\left(\mathrm{CDCl}_{3}\right)$

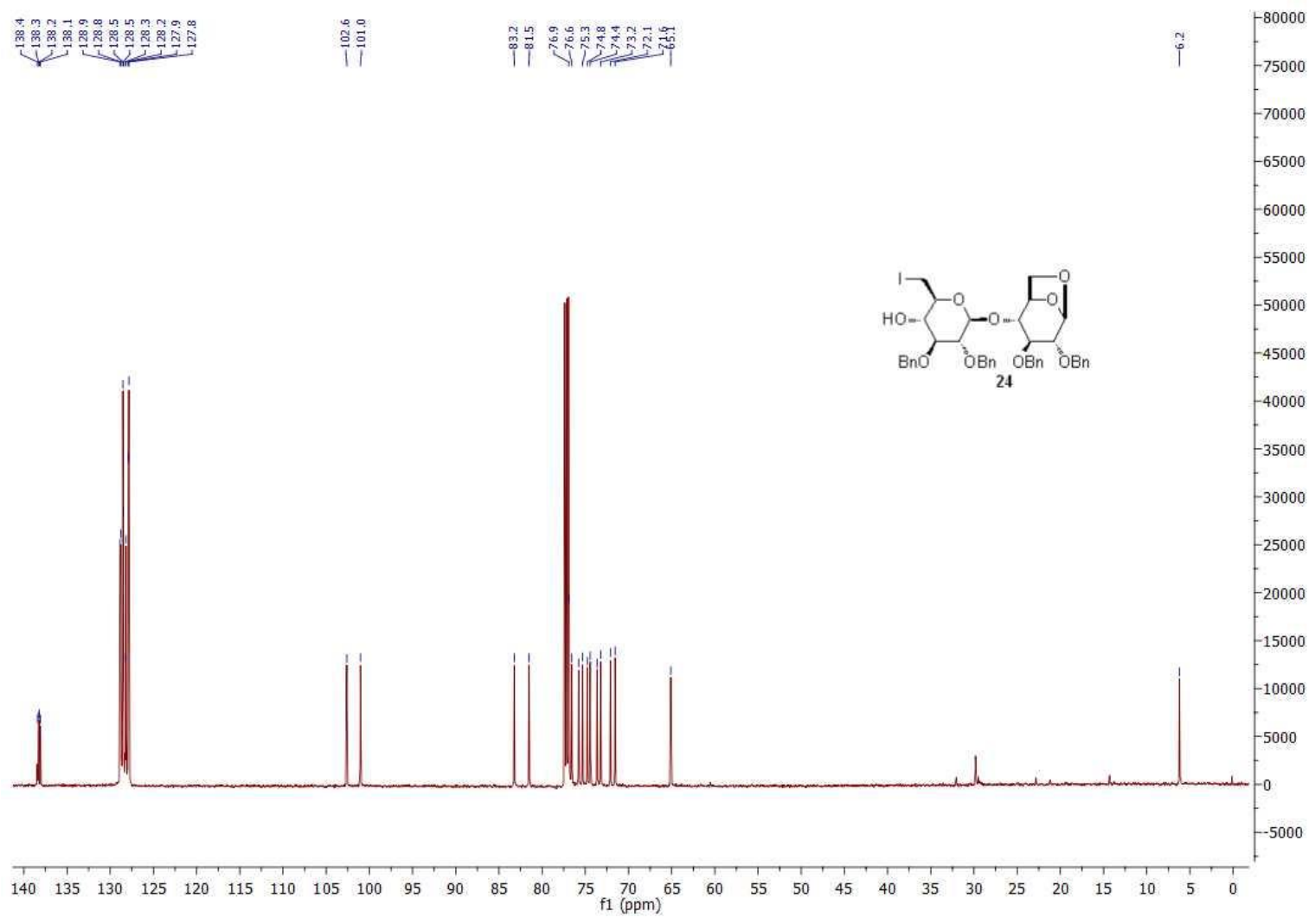

${ }^{13} \mathrm{C}-\mathrm{NMR}$ Spectrum of Compound $24\left(\mathrm{CDCl}_{3}\right)$ 


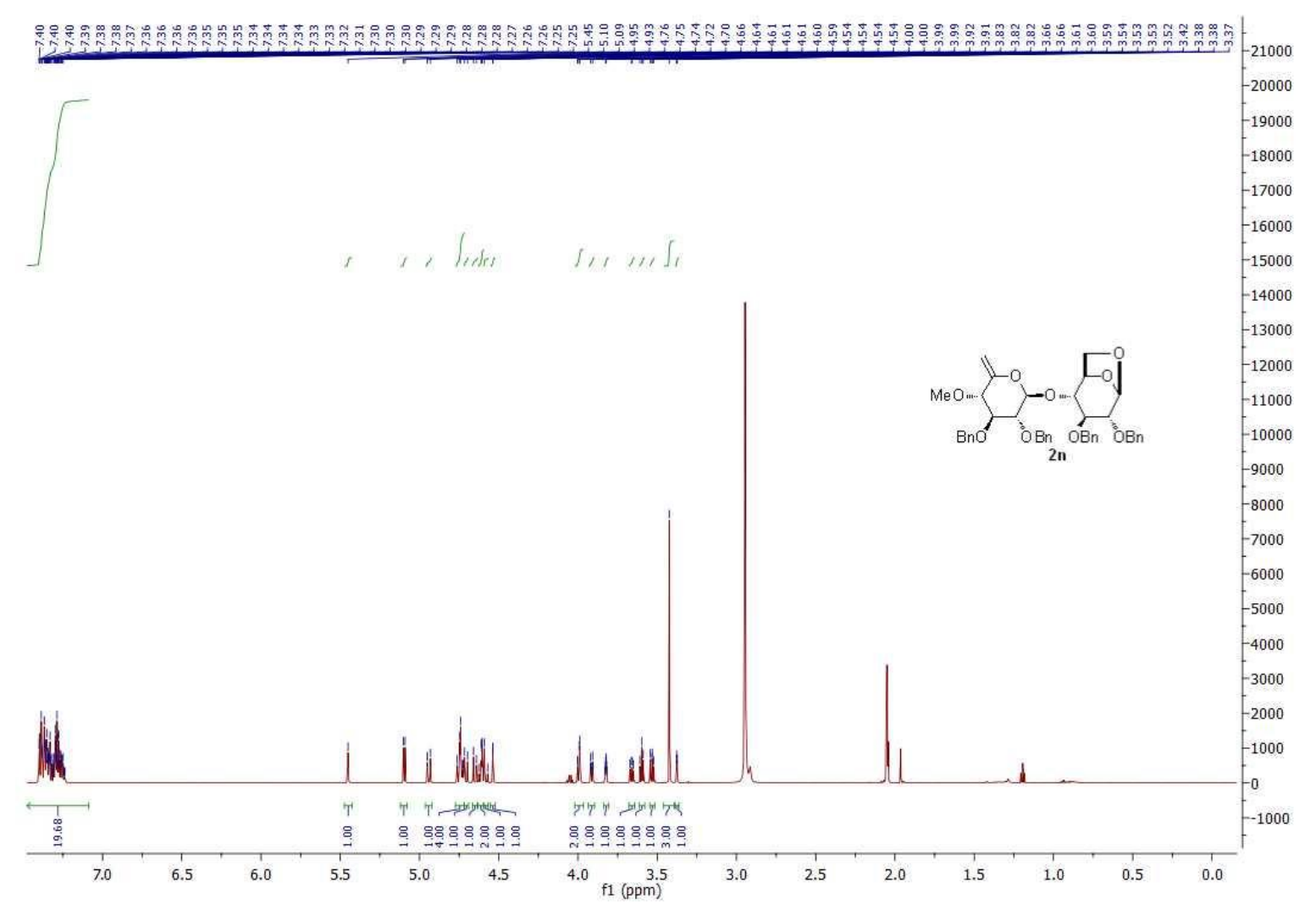

${ }^{1}$ H-NMR Spectrum of Compound 2n (Acetone-d6)

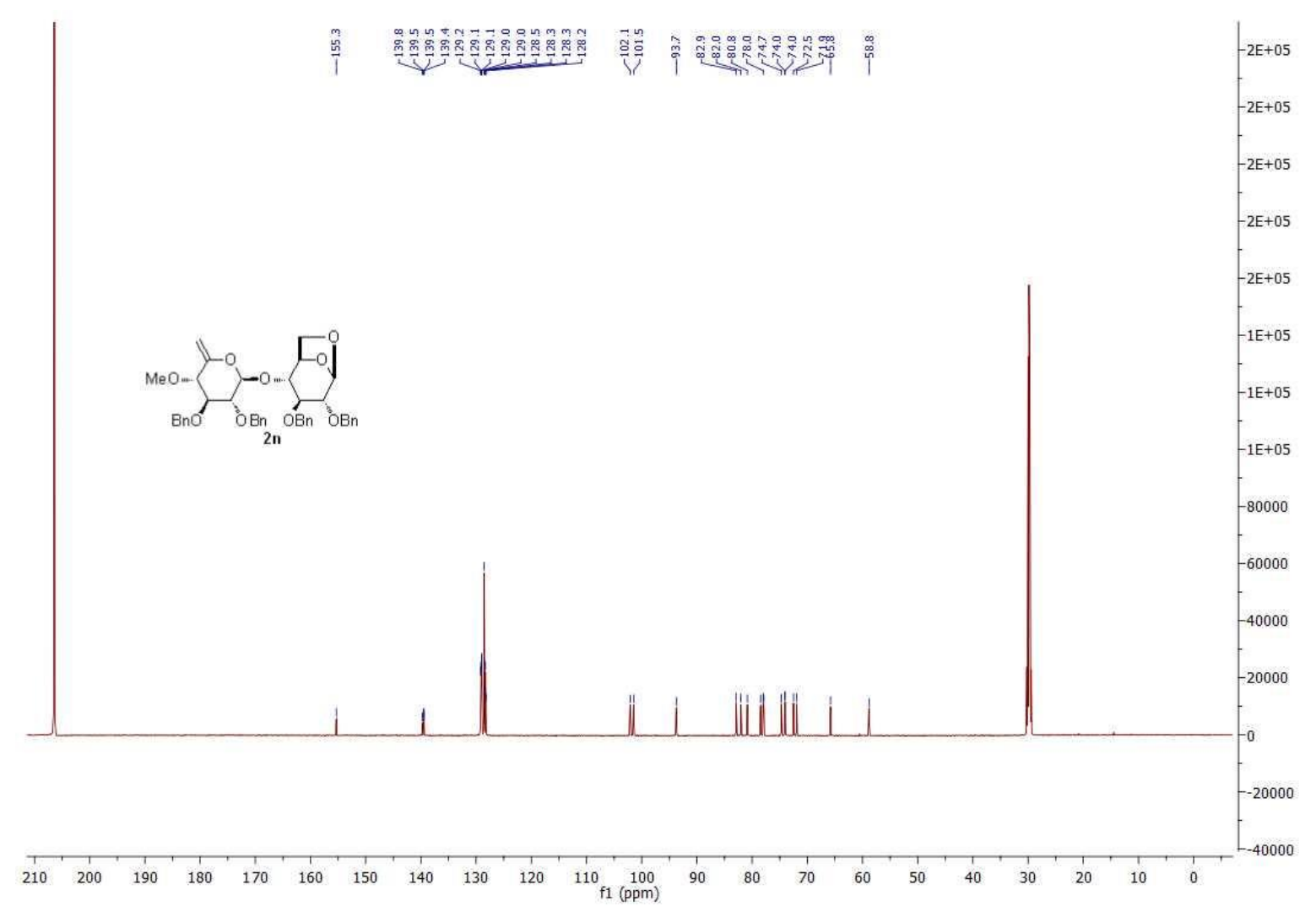

${ }^{13}$ C-NMR Spectrum of Compound 2n (Acetone-d6) 


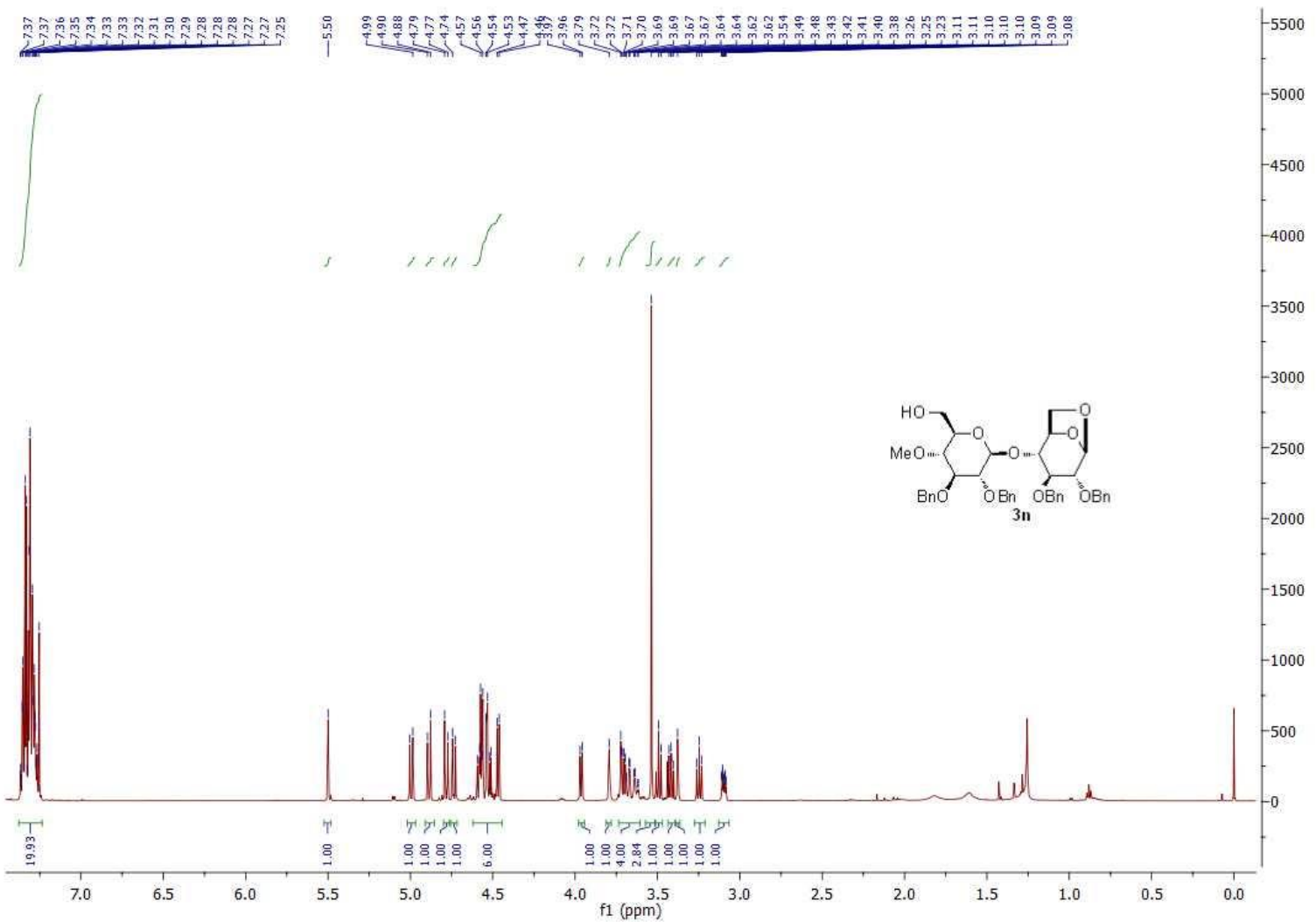

${ }^{1} \mathrm{H}-\mathrm{NMR}$ Spectrum of Compound $\mathbf{3 n}\left(\mathrm{CDCl}_{3}\right)$

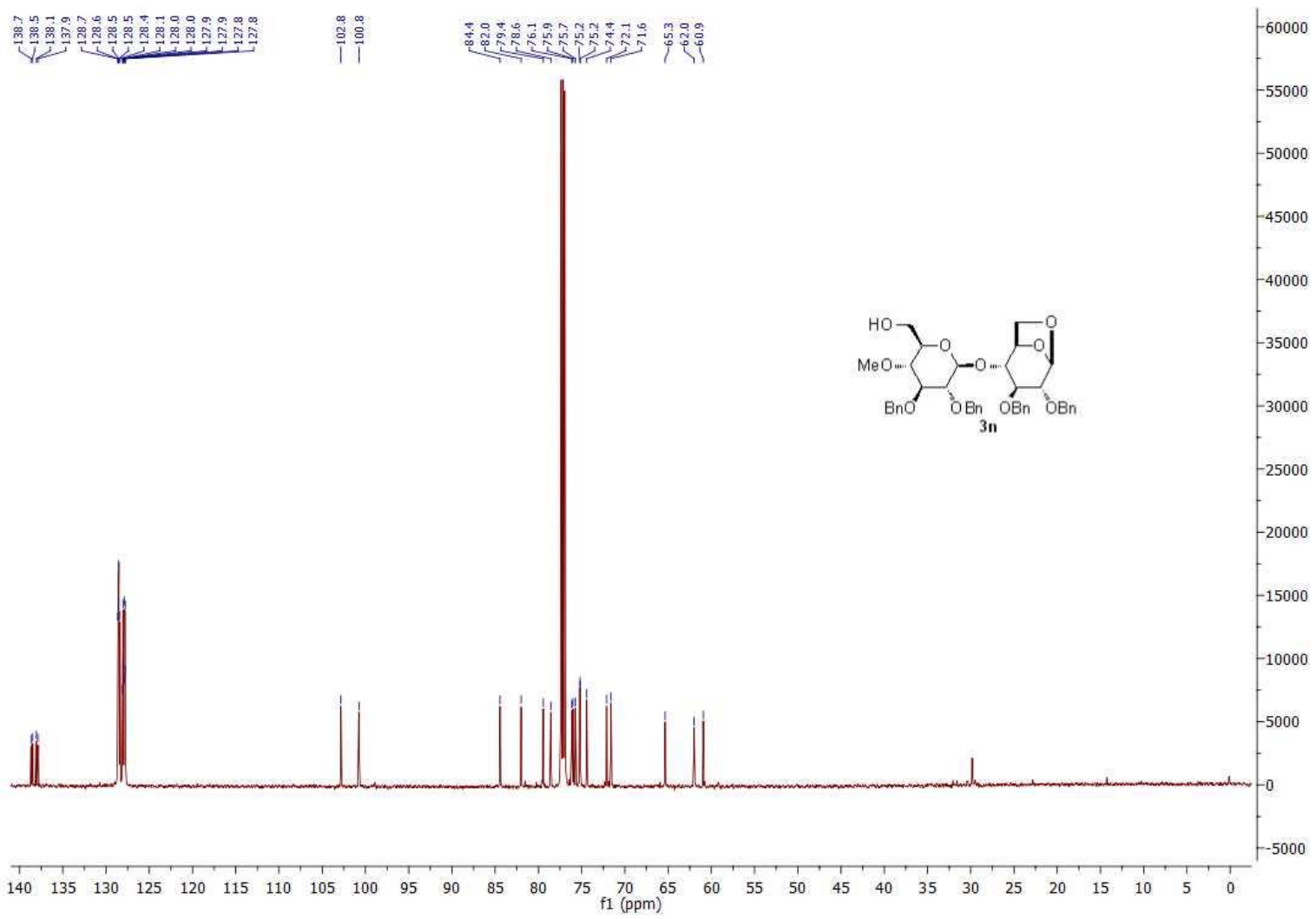

${ }^{13} \mathrm{C}-\mathrm{NMR}$ Spectrum of Compound $\mathbf{3 n}\left(\mathrm{CDCl}_{3}\right)$ 


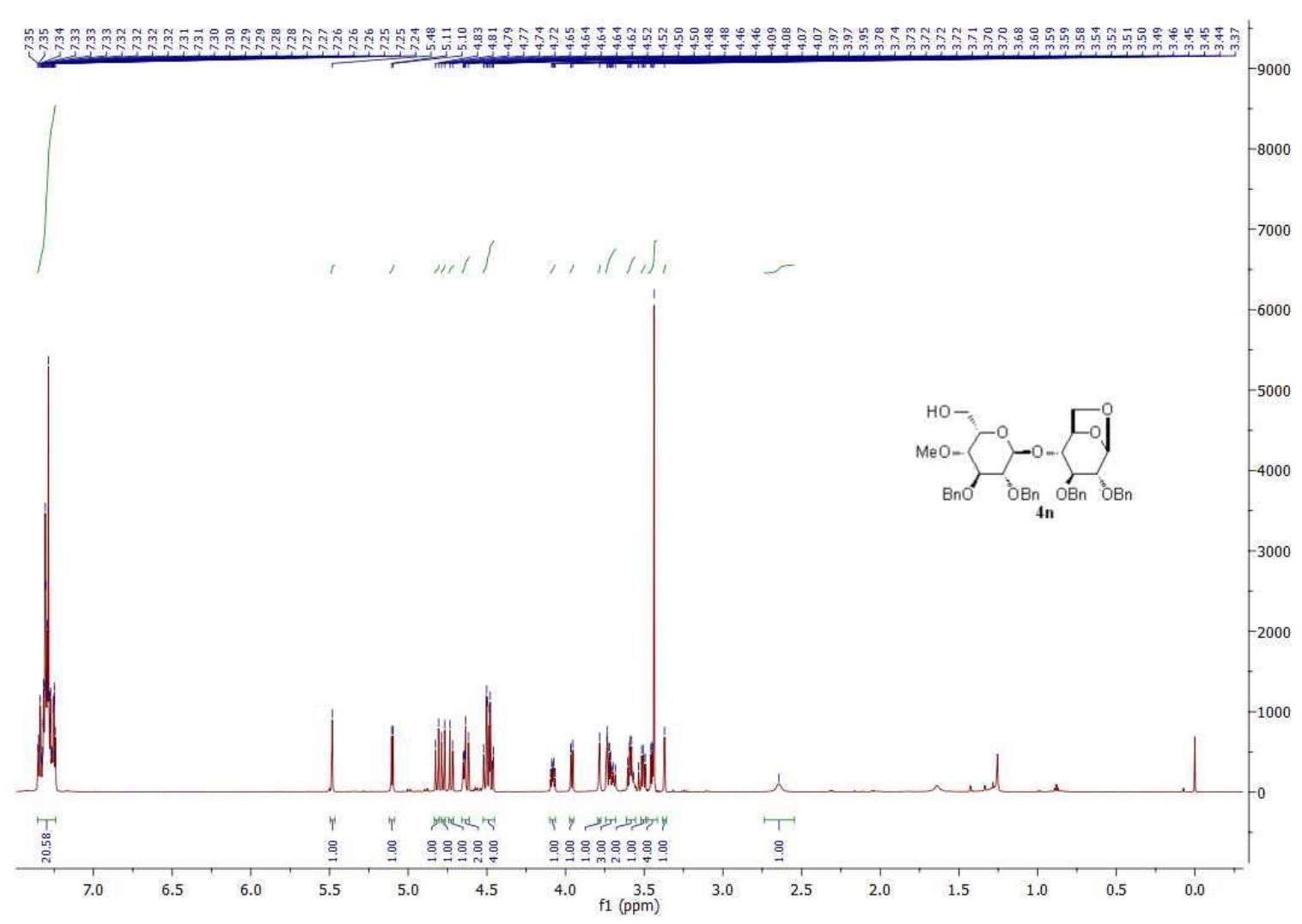

${ }^{1} \mathrm{H}-\mathrm{NMR}$ Spectrum of Compound $\mathbf{4 n}\left(\mathrm{CDCl}_{3}\right)$

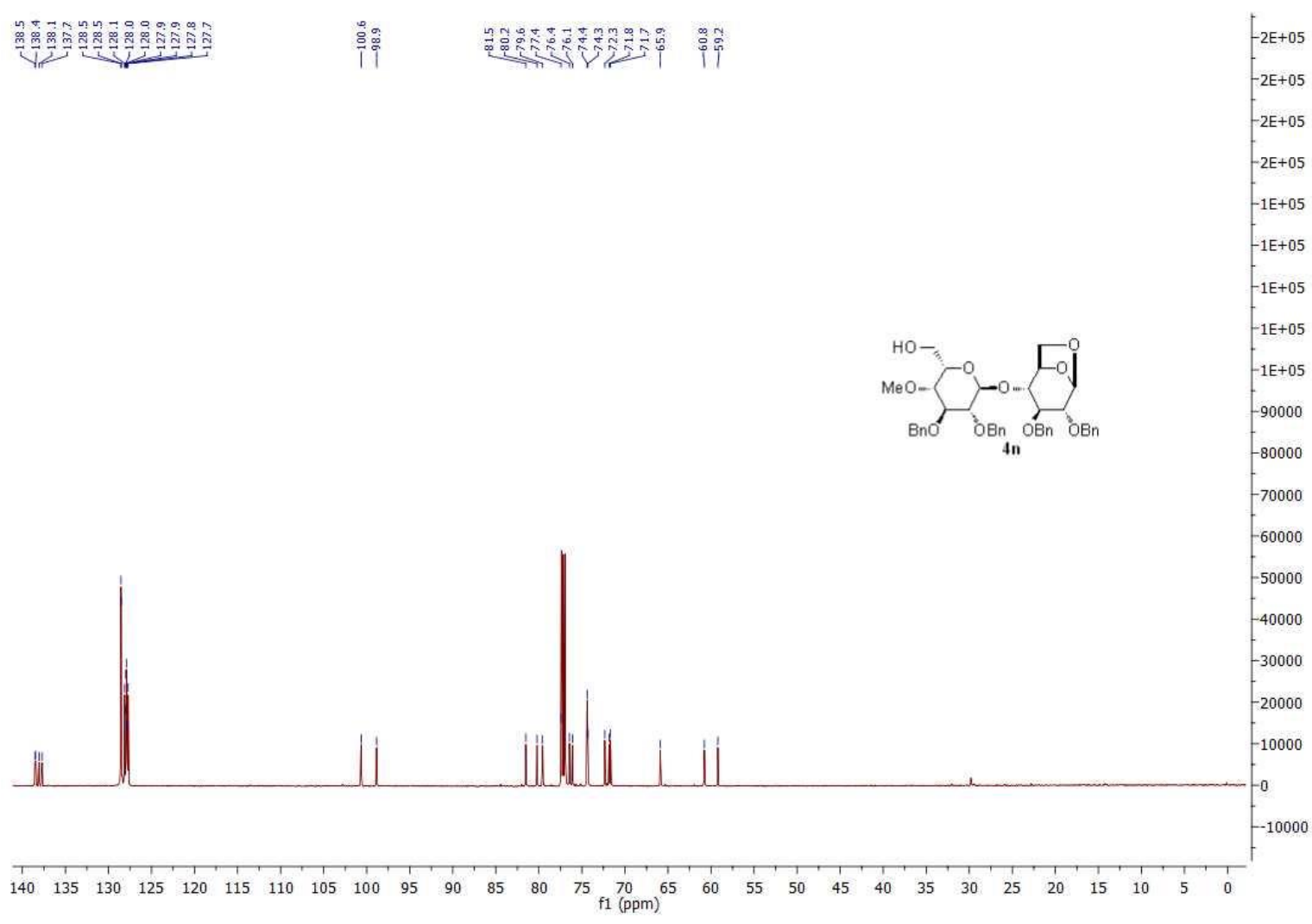

${ }^{13} \mathrm{C}-\mathrm{NMR}$ Spectrum of Compound $4 \mathbf{n}\left(\mathrm{CDCl}_{3}\right)$ 


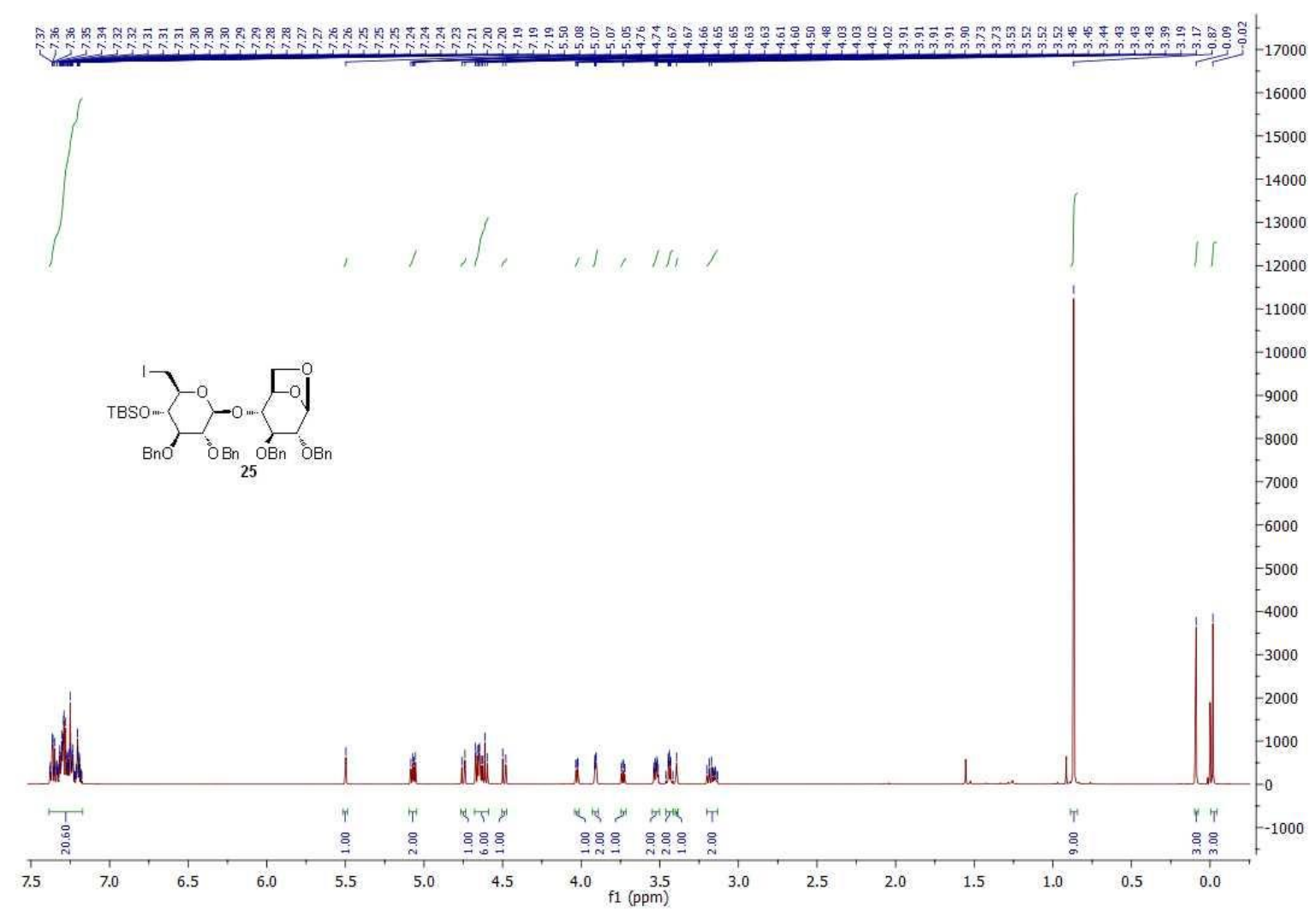

${ }^{1} \mathrm{H}-\mathrm{NMR}$ Spectrum of Compound $25\left(\mathrm{CDCl}_{3}\right)$

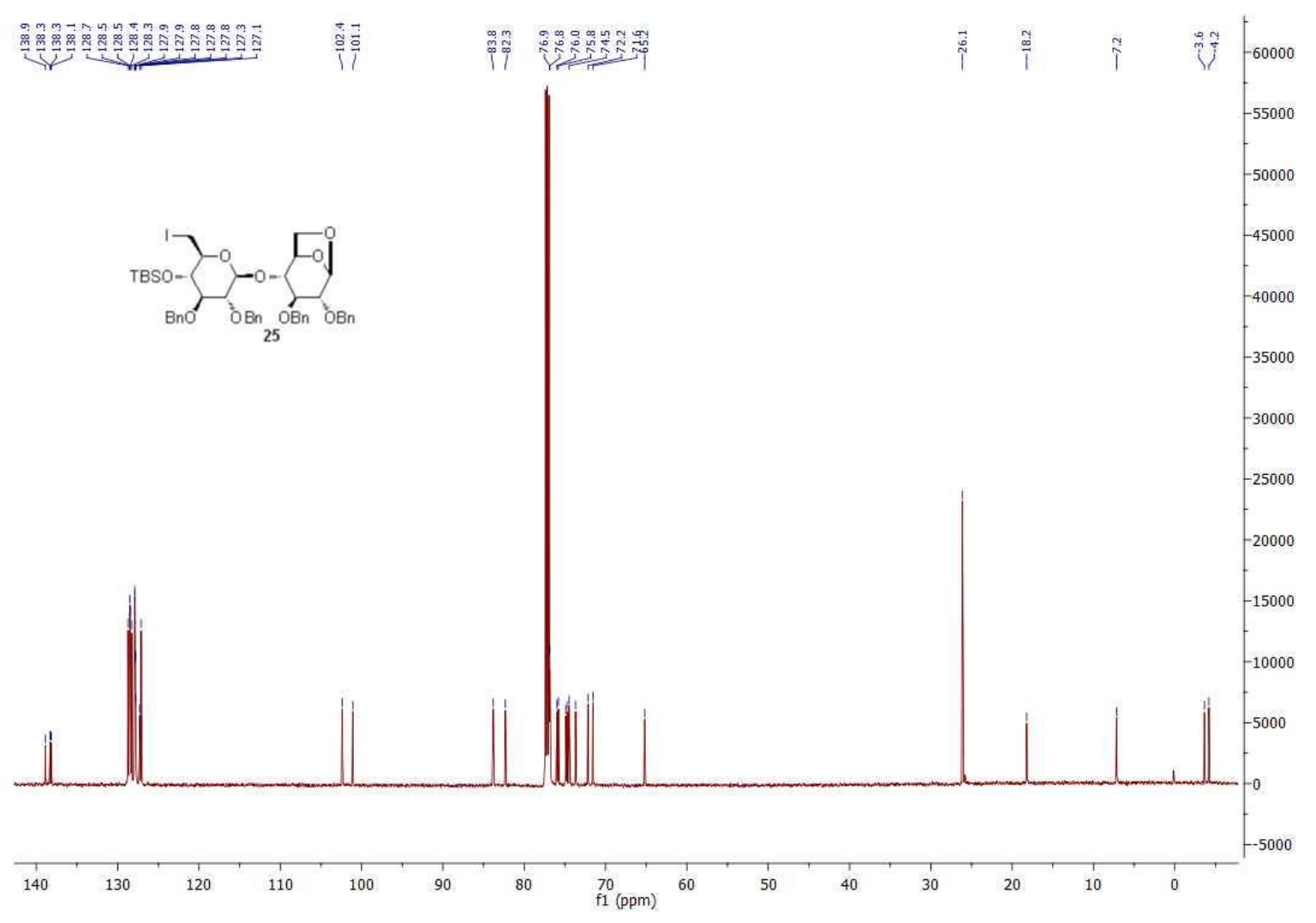

${ }^{13} \mathrm{C}-\mathrm{NMR}$ Spectrum of Compound $25\left(\mathrm{CDCl}_{3}\right)$ 


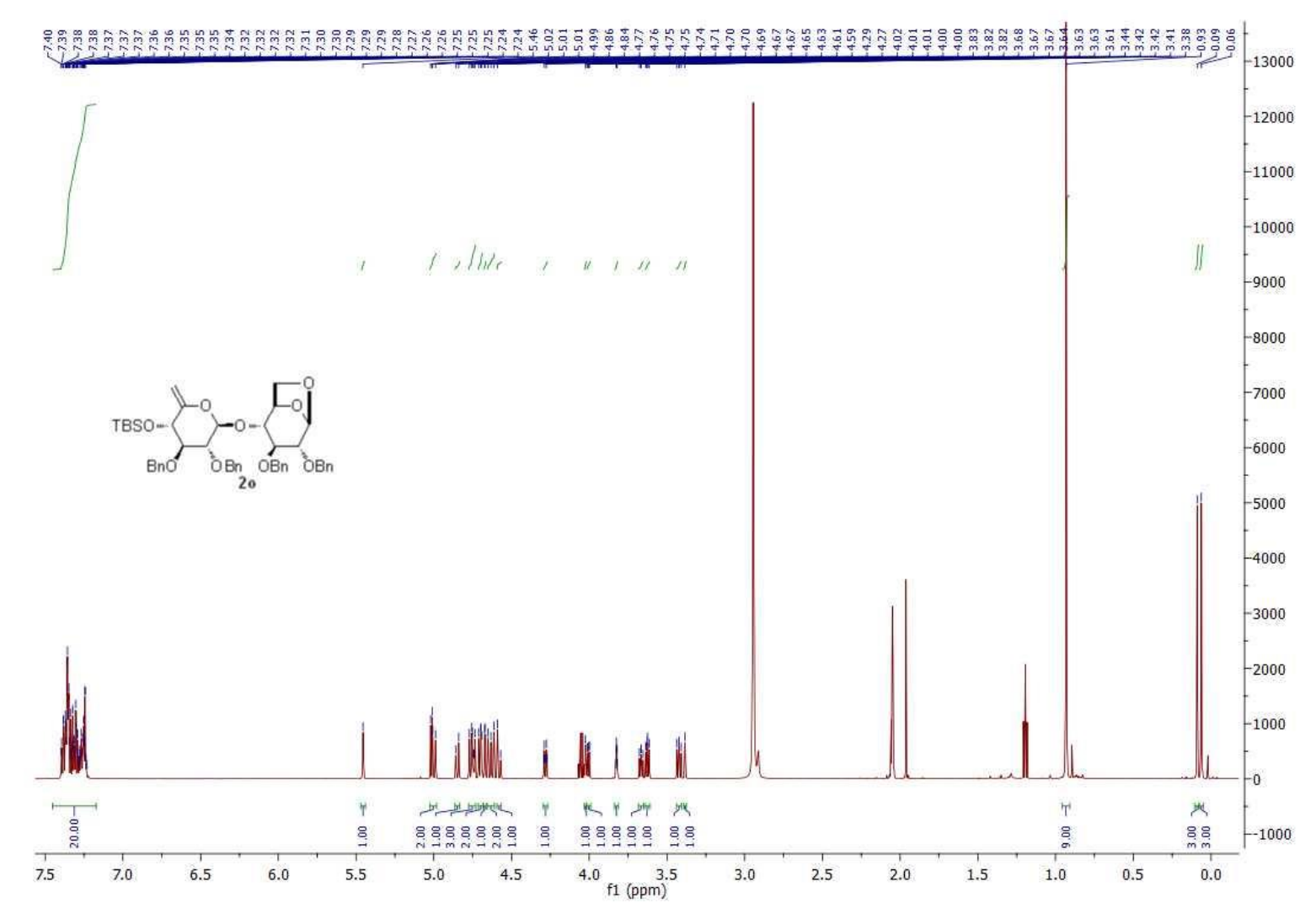

${ }^{1}$ H-NMR Spectrum of Compound 20 (Acetone-d6)

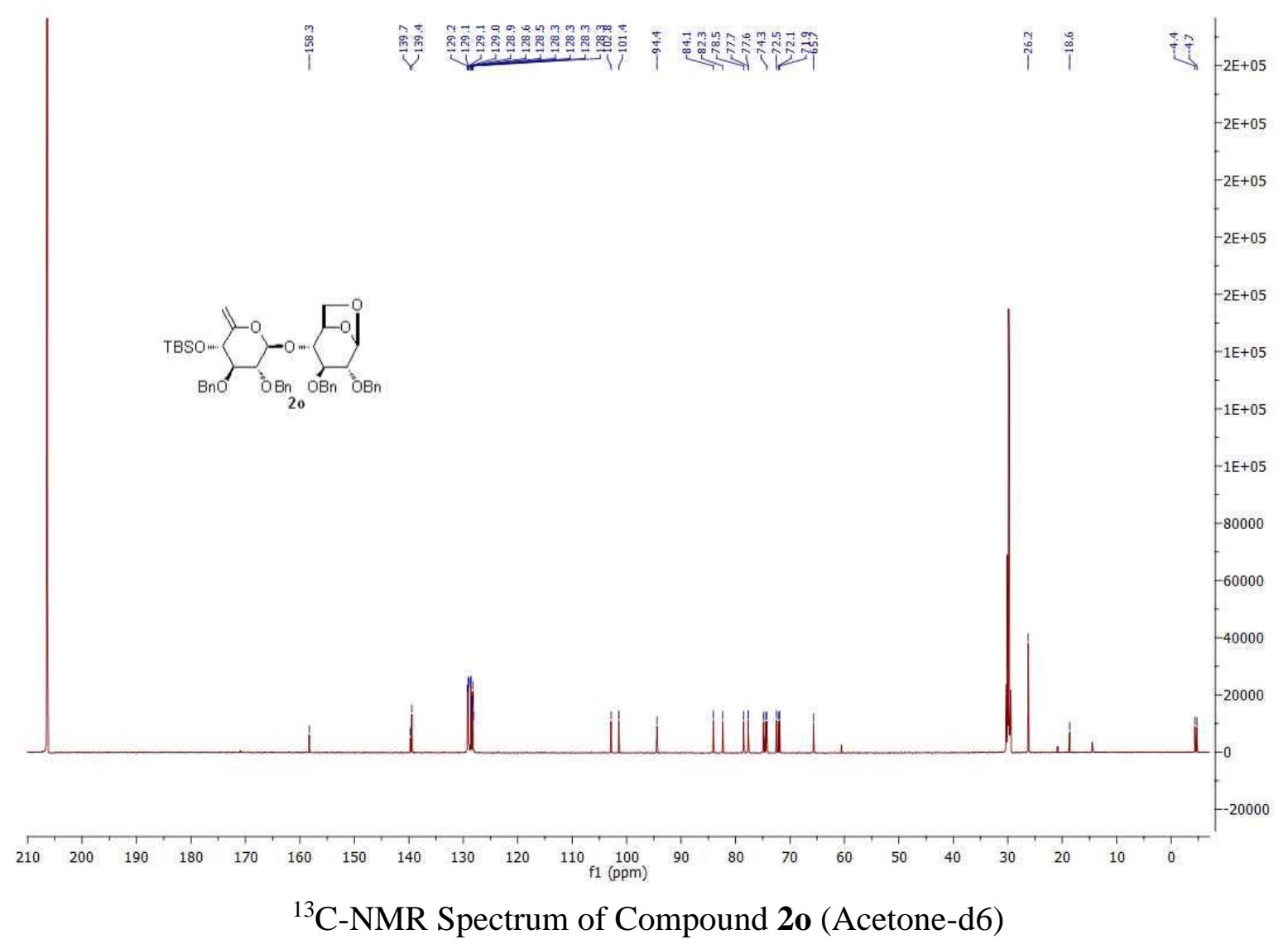




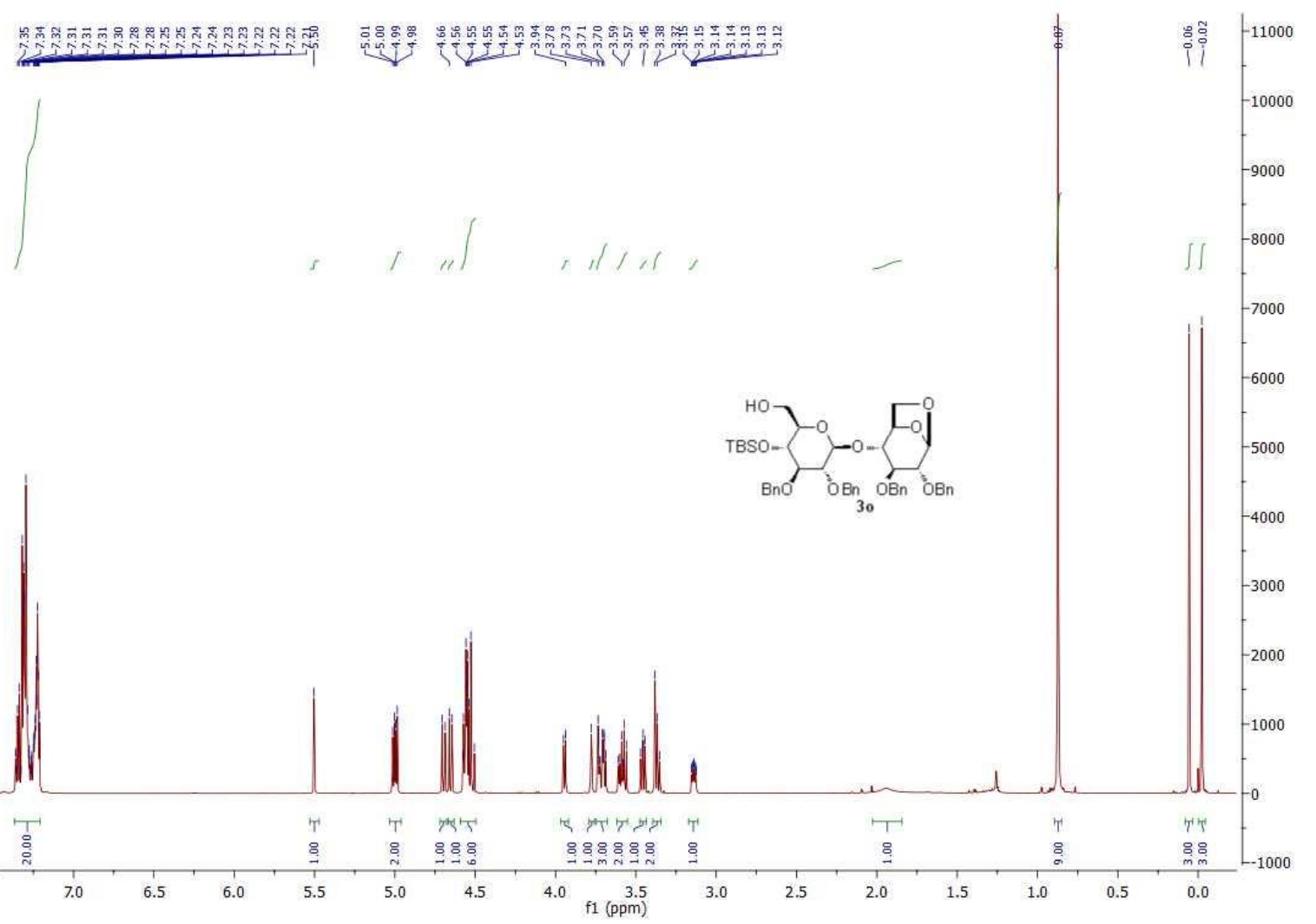

${ }^{1} \mathrm{H}-\mathrm{NMR}$ Spectrum of Compound $\mathbf{3 o}\left(\mathrm{CDCl}_{3}\right)$

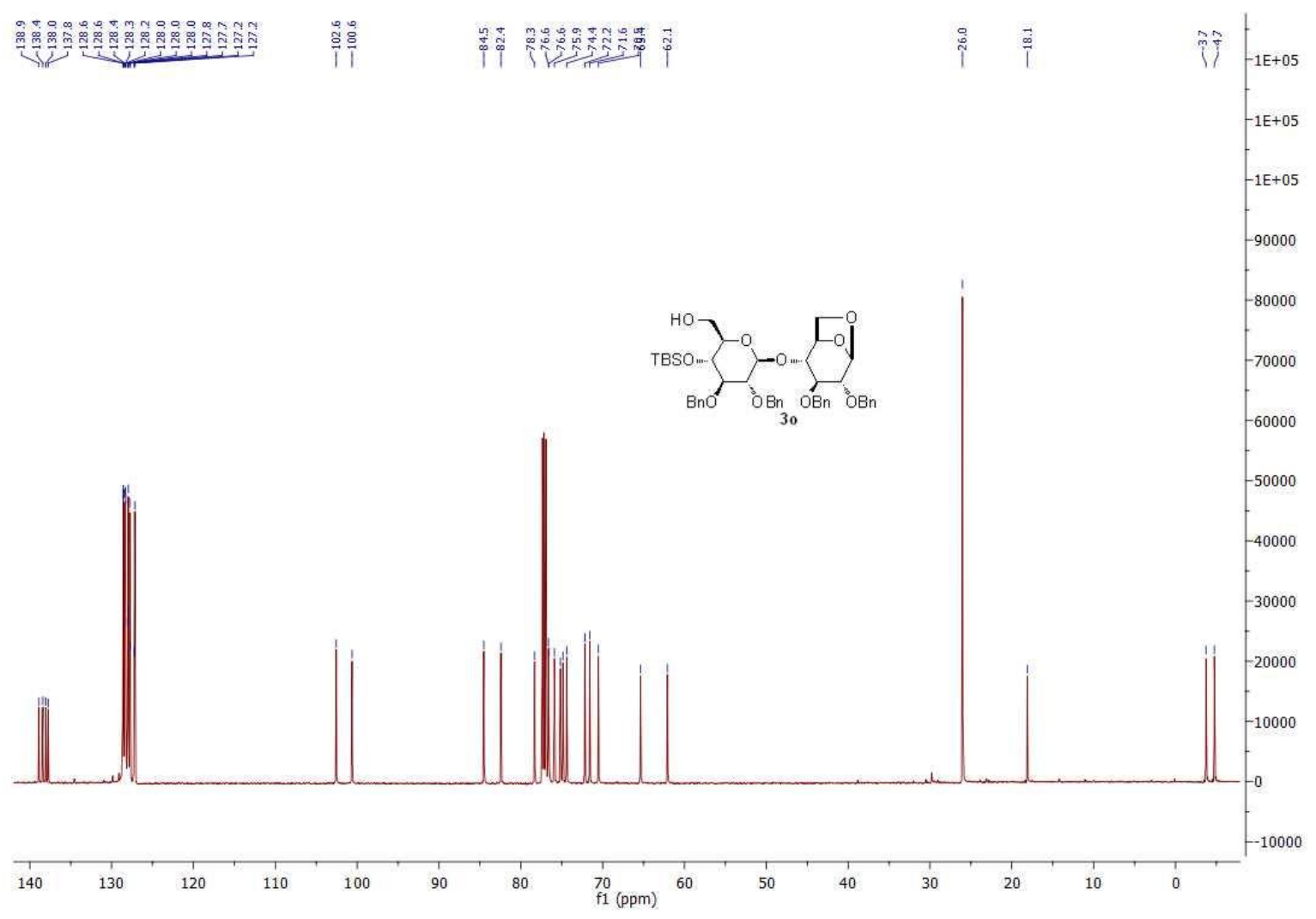

${ }^{13} \mathrm{C}$-NMR Spectrum of Compound $\mathbf{3 o}\left(\mathrm{CDCl}_{3}\right)$ 


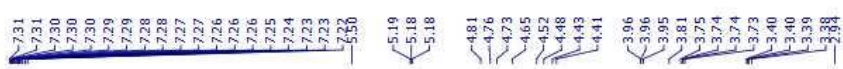

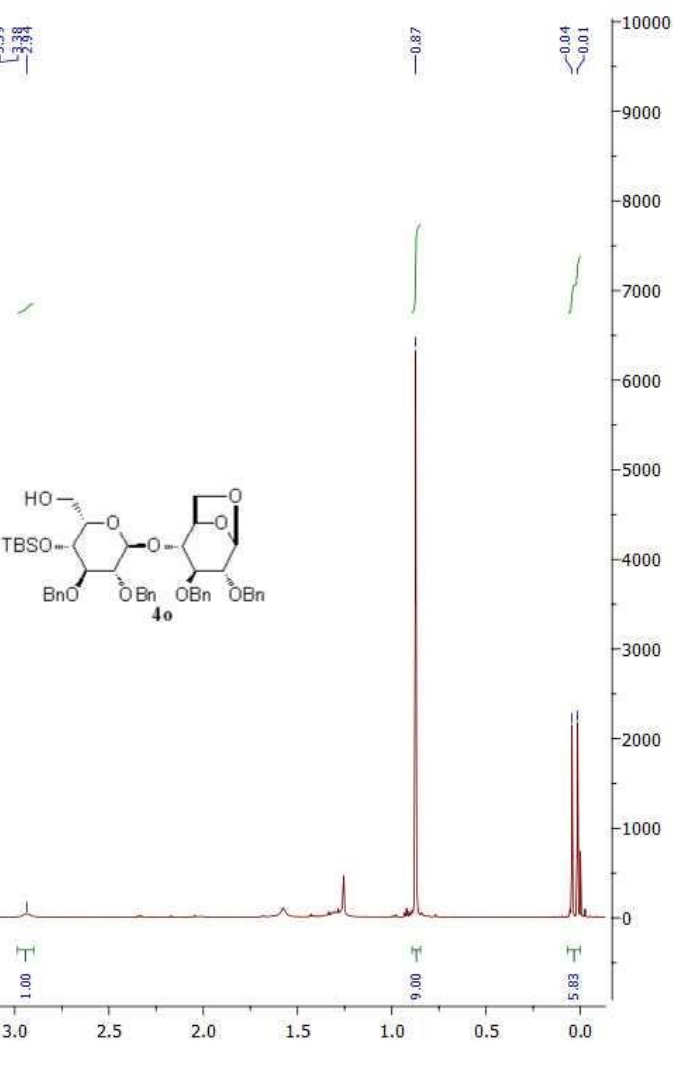

${ }^{1} \mathrm{H}-\mathrm{NMR}$ Spectrum of Compound $\mathbf{4 o}\left(\mathrm{CDCl}_{3}\right)$

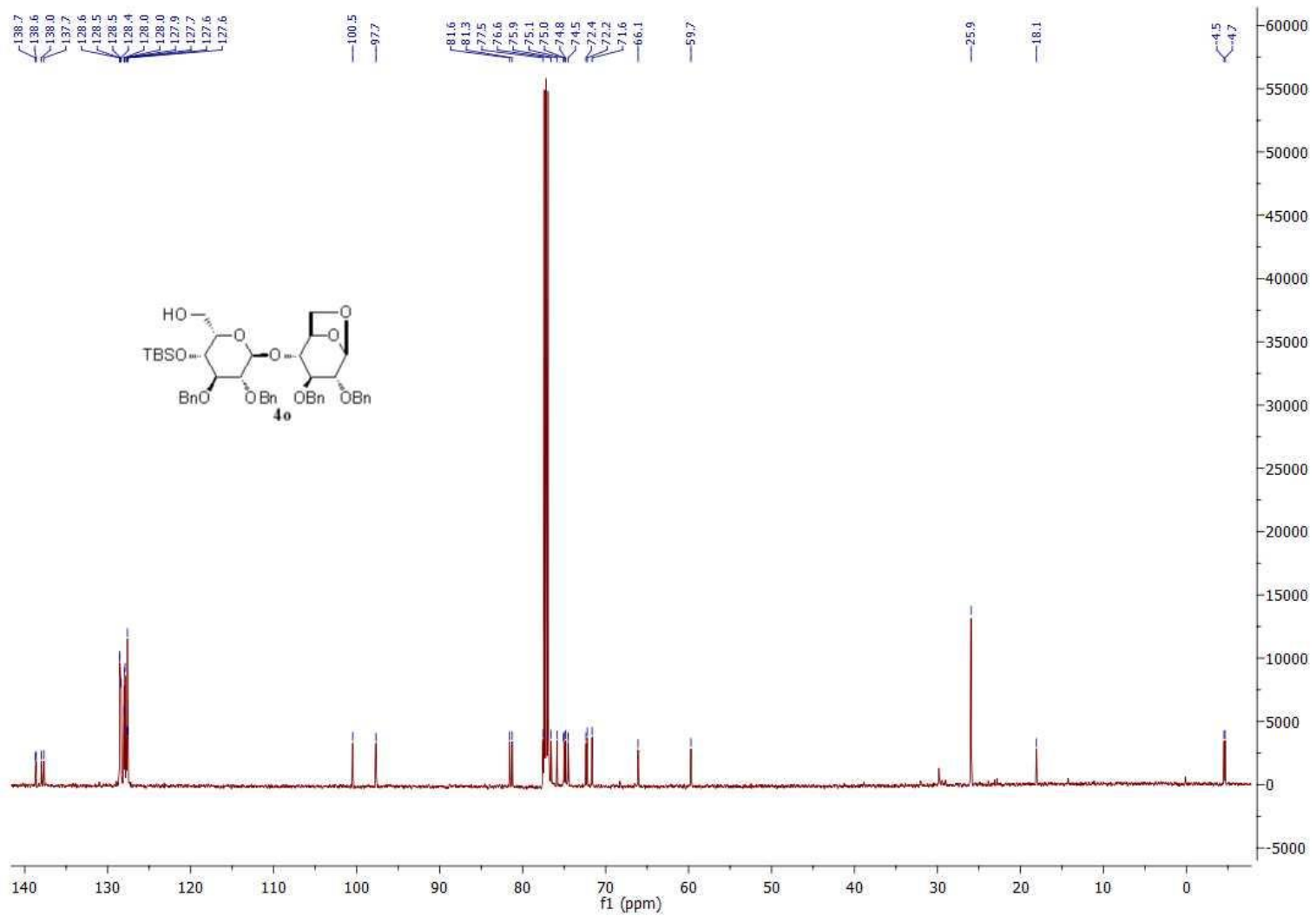

${ }^{13} \mathrm{C}$-NMR Spectrum of Compound $4 \mathbf{o}\left(\mathrm{CDCl}_{3}\right)$ 


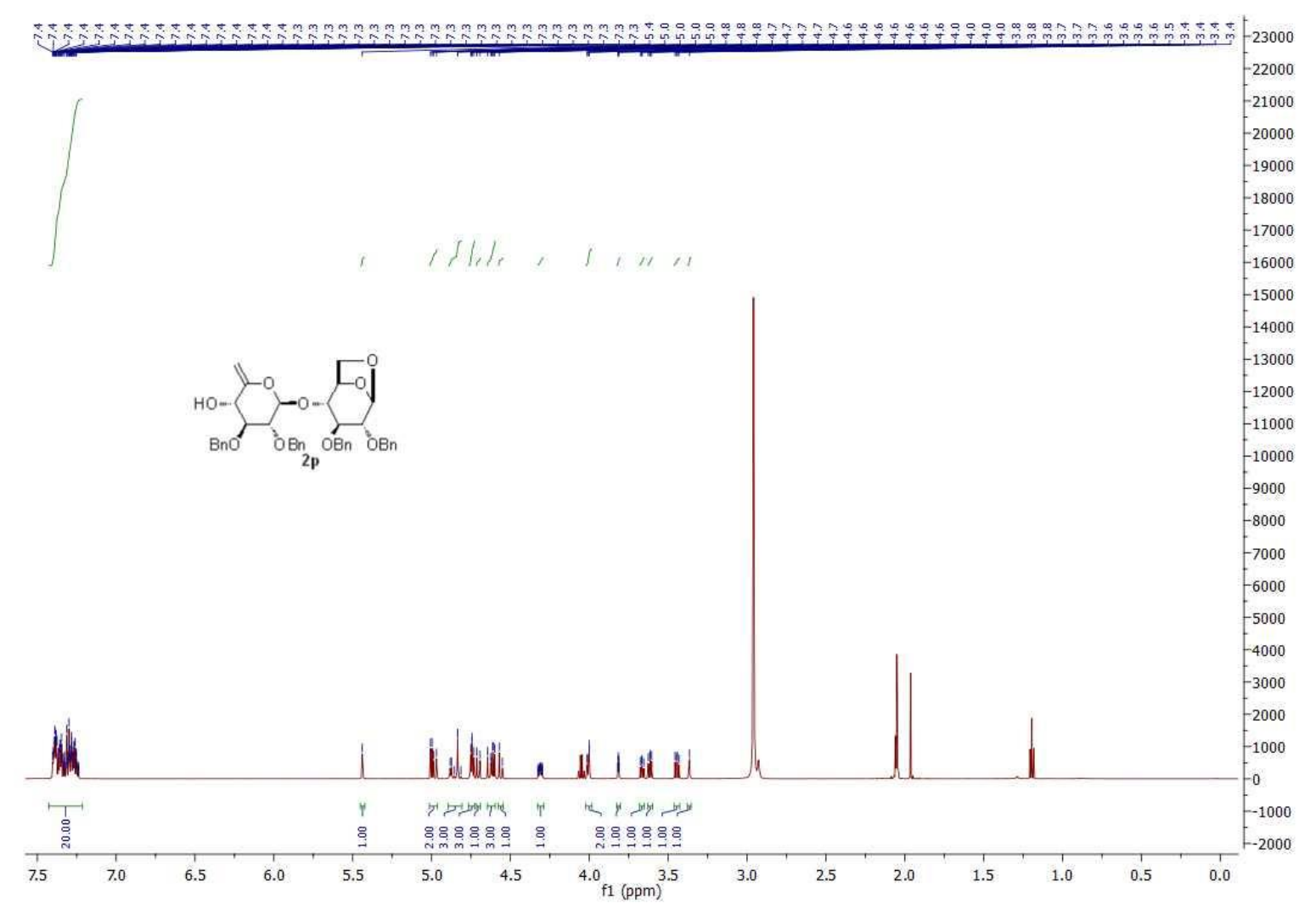

${ }^{1}$ H-NMR Spectrum of Compound 2p (Acetone-d6)

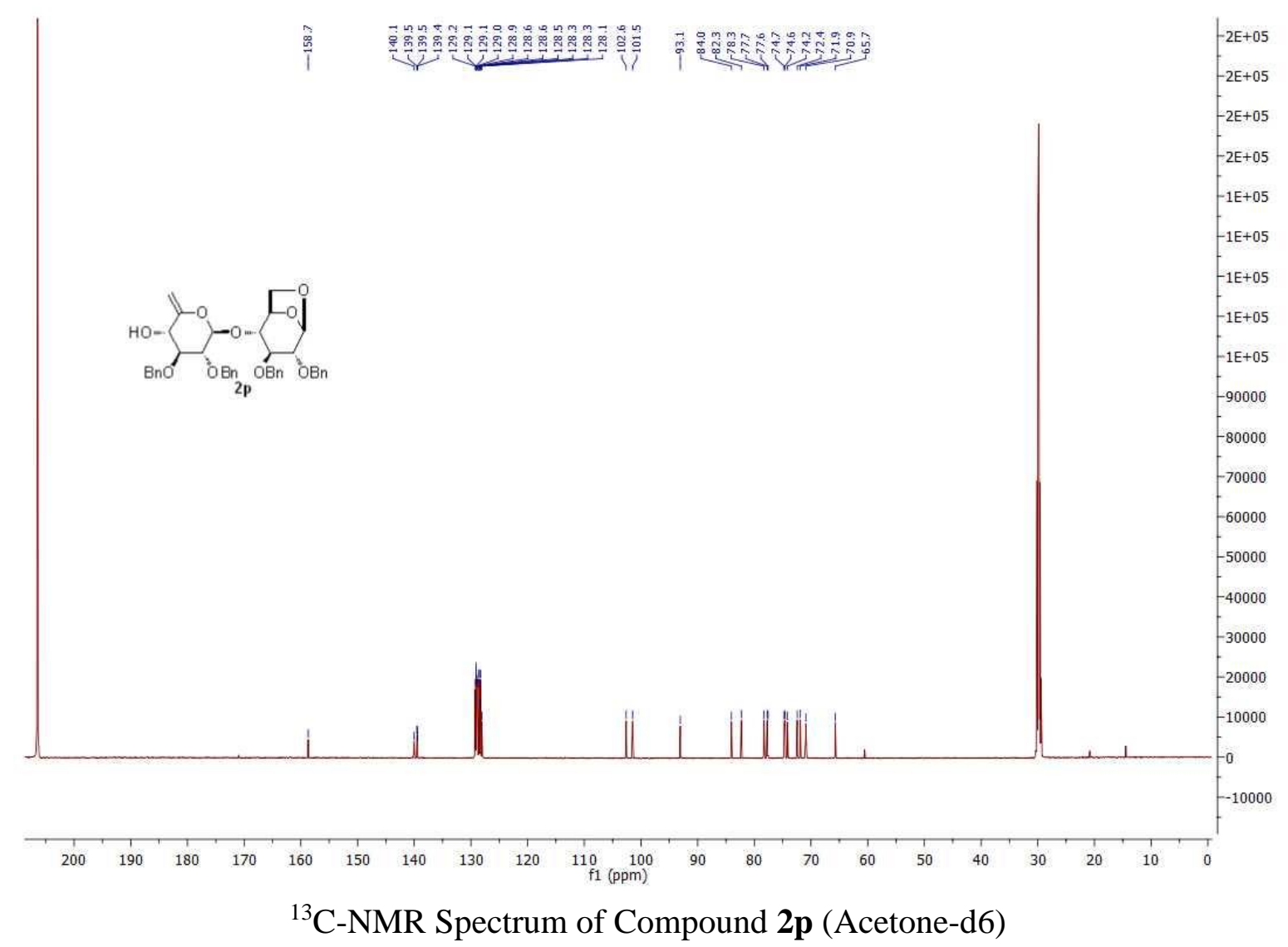




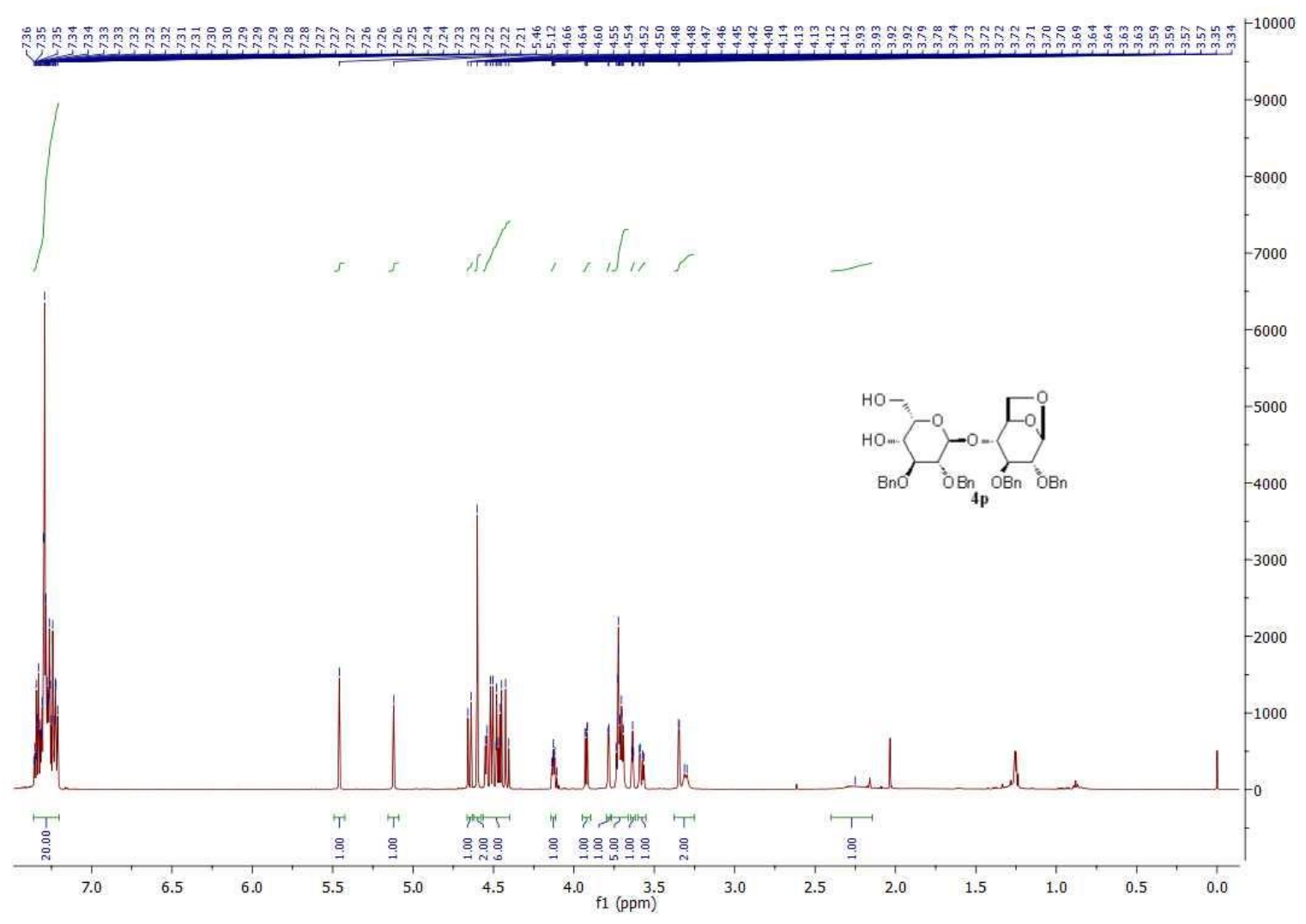

${ }^{1} \mathrm{H}-\mathrm{NMR}$ Spectrum of Compound $\mathbf{4 p}\left(\mathrm{CDCl}_{3}\right)$

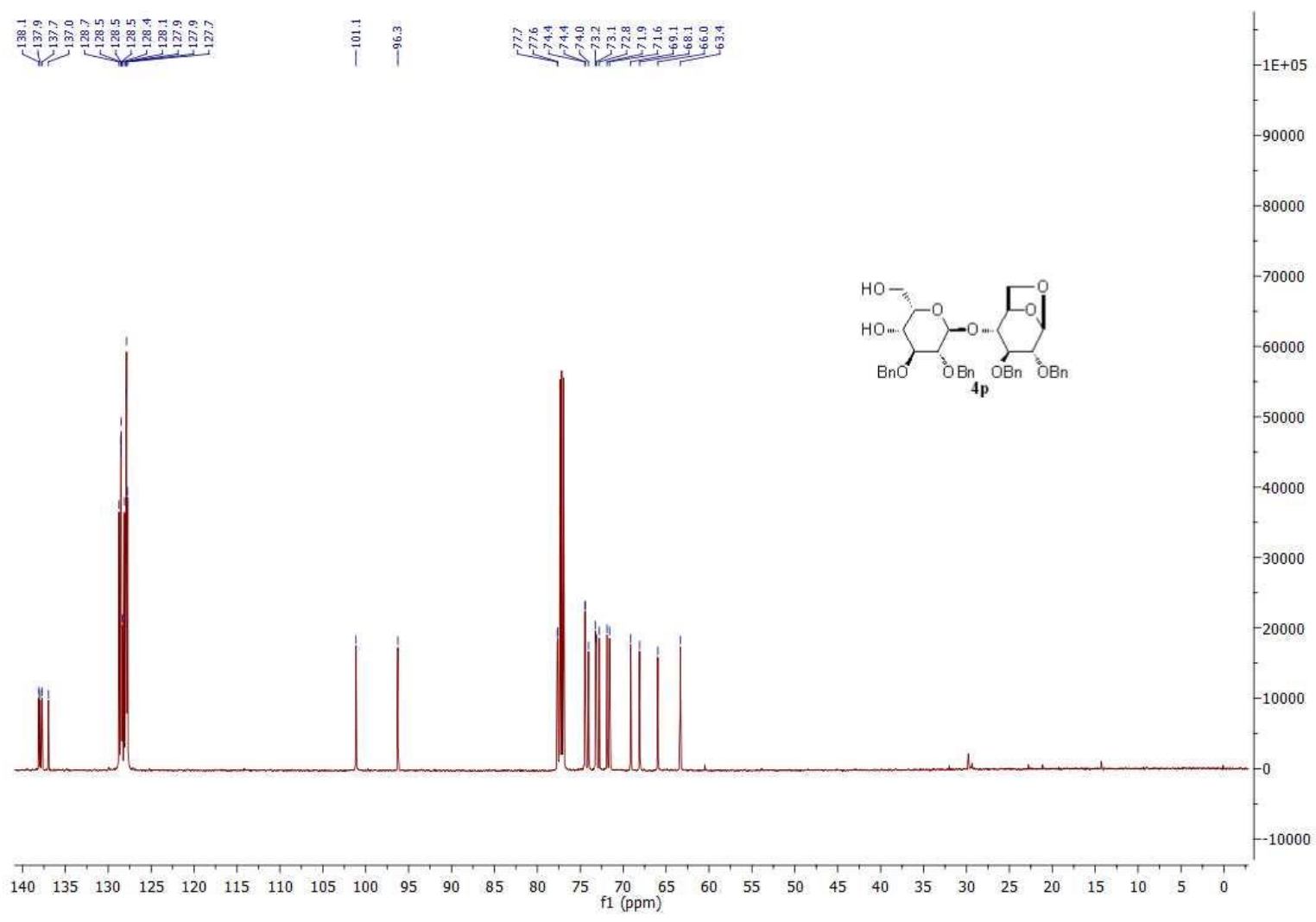

${ }^{13} \mathrm{C}-\mathrm{NMR}$ Spectrum of Compound $4 \mathbf{p}\left(\mathrm{CDCl}_{3}\right)$ 


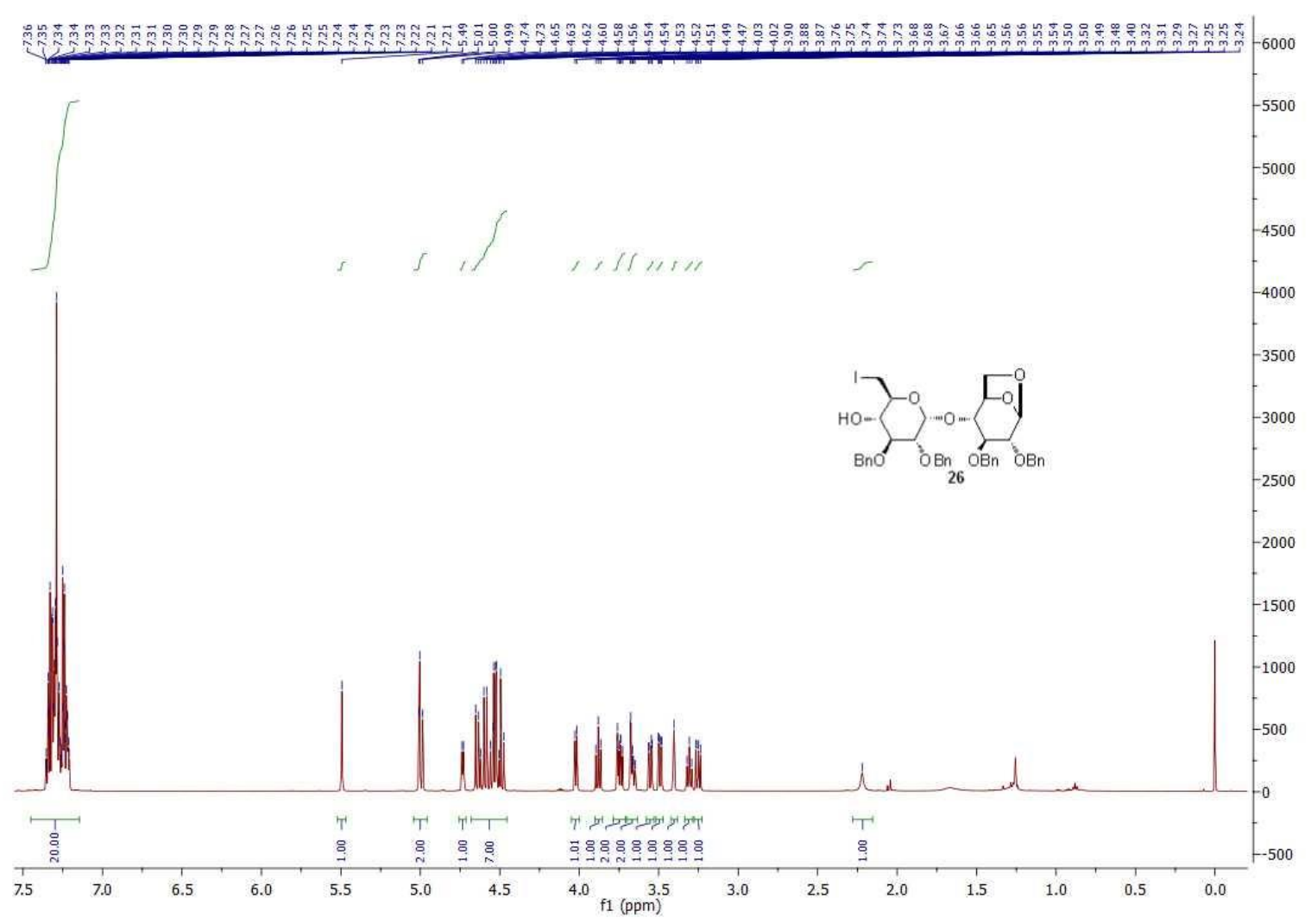

${ }^{1} \mathrm{H}-\mathrm{NMR}$ Spectrum of Compound $\mathbf{2 6}\left(\mathrm{CDCl}_{3}\right)$

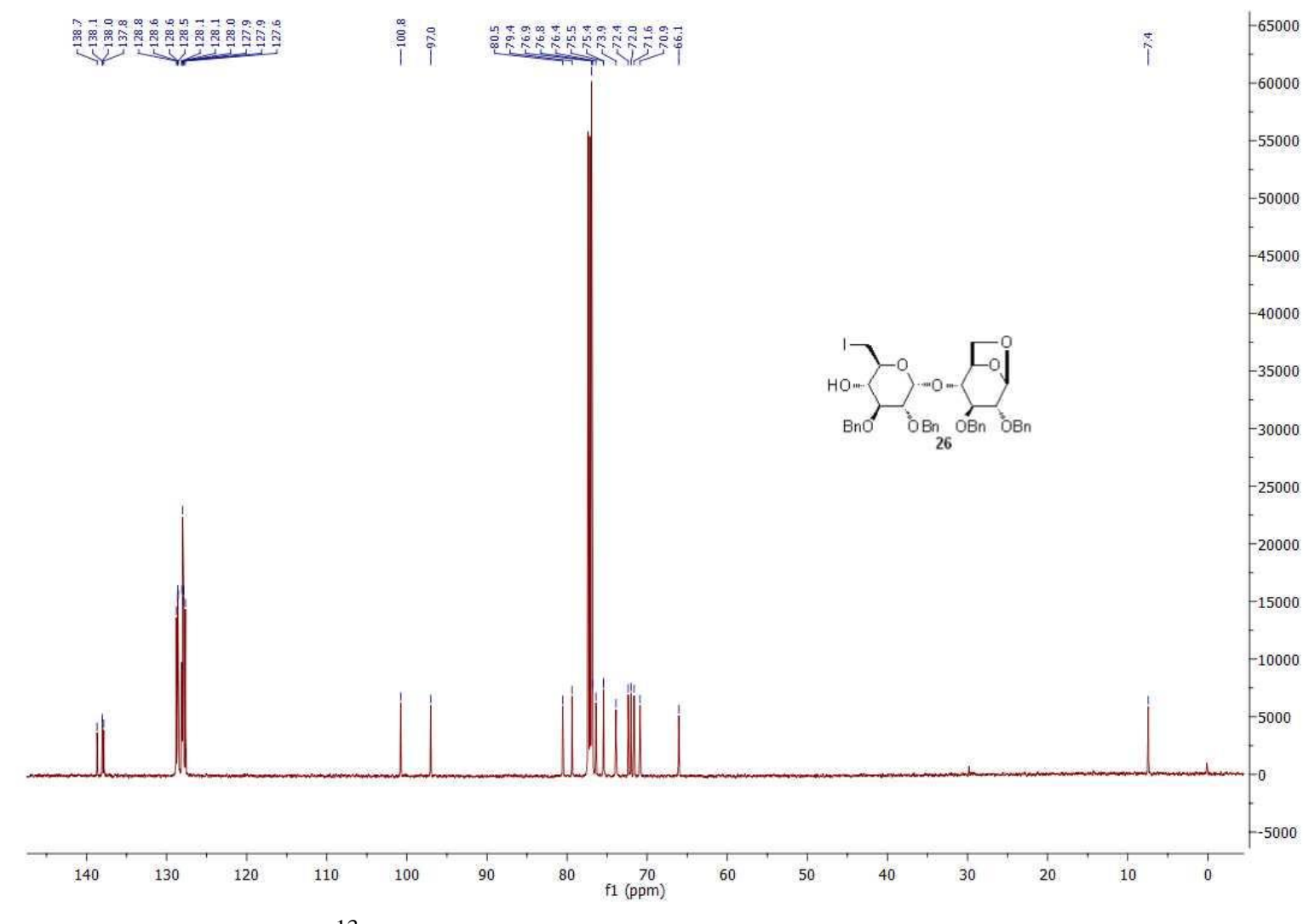

${ }^{13} \mathrm{C}-\mathrm{NMR}$ Spectrum of Compound $26\left(\mathrm{CDCl}_{3}\right)$ 


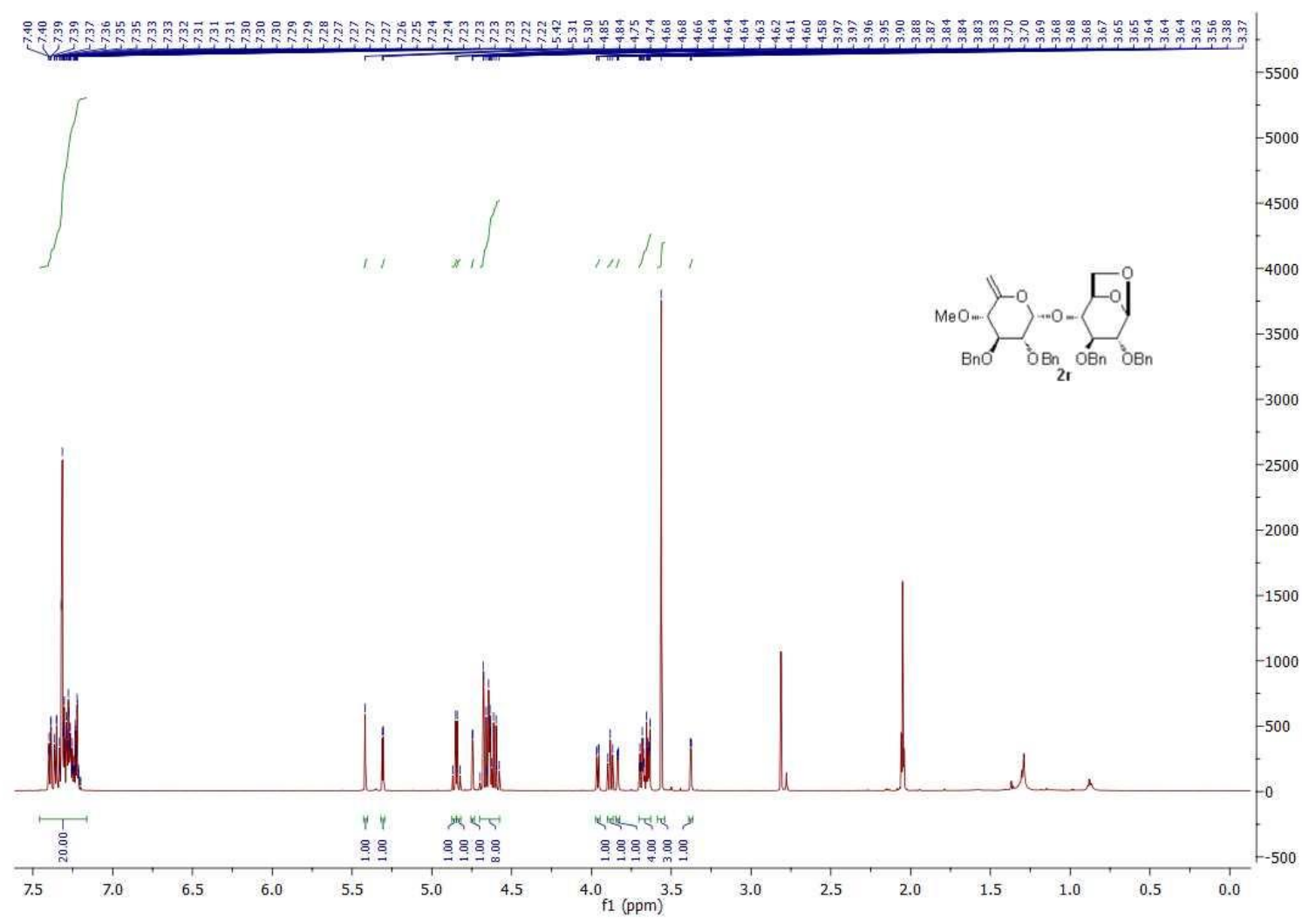

${ }^{1}$ H-NMR Spectrum of Compound 2r (Acetone-d6)

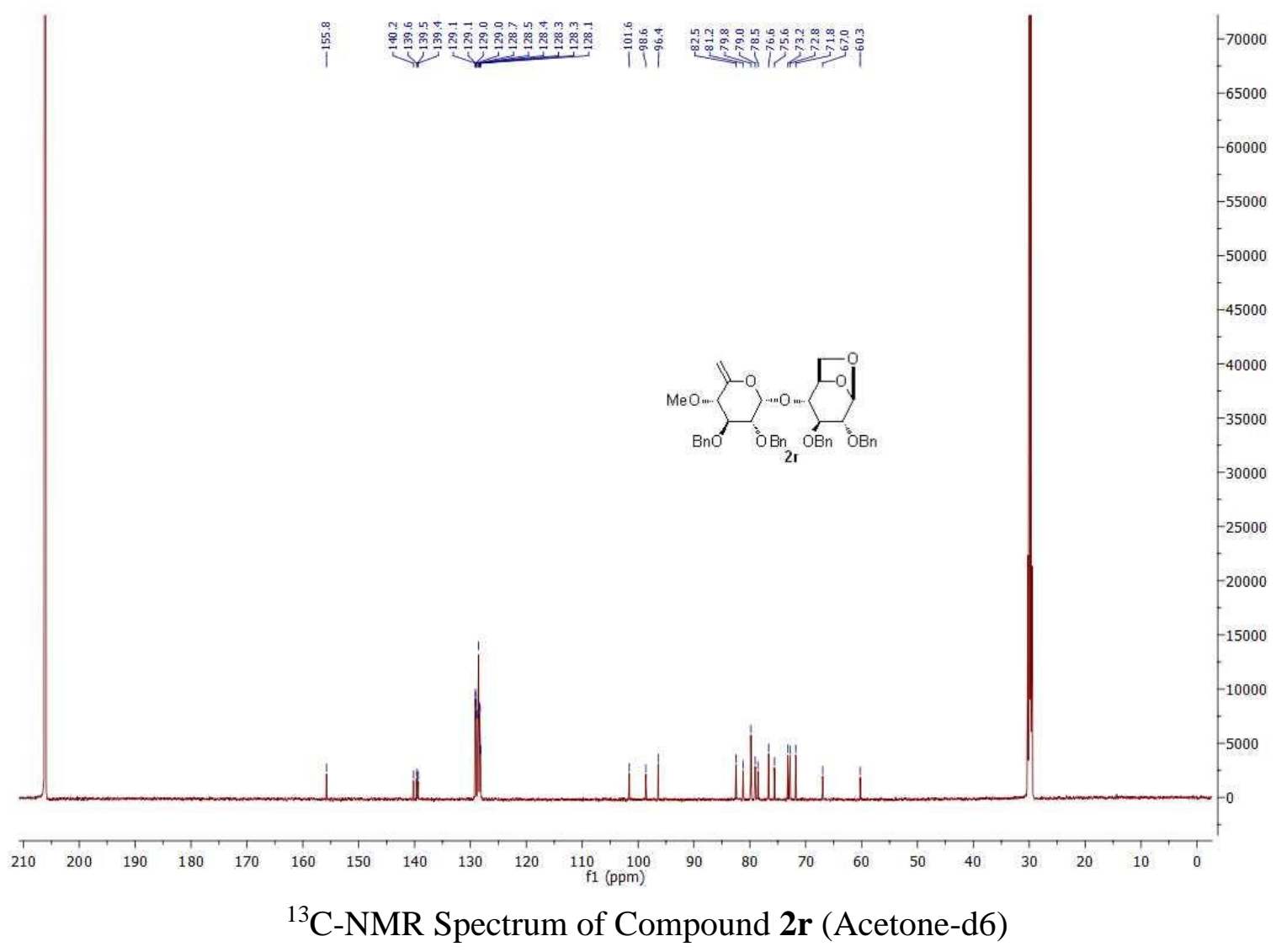




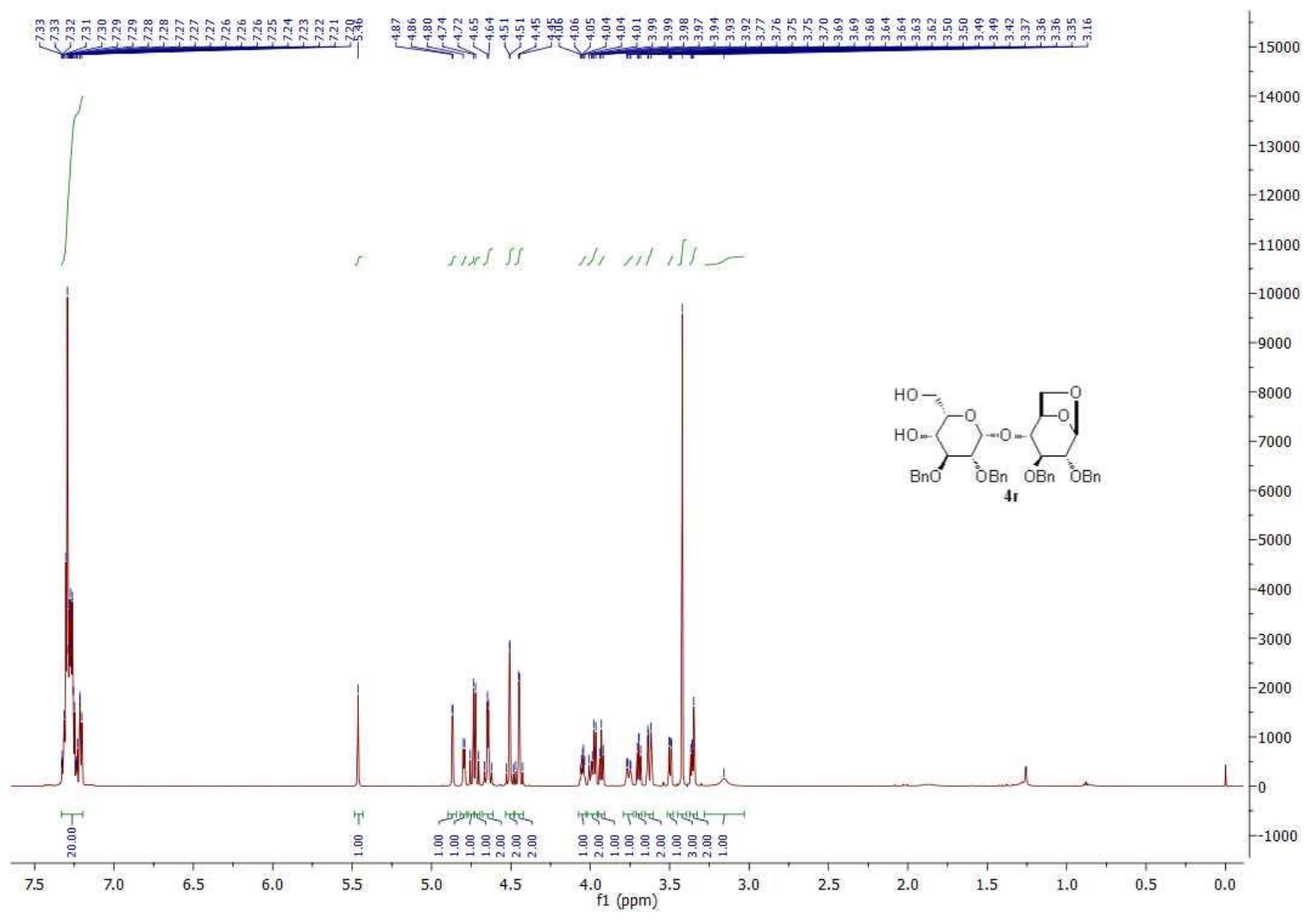

${ }^{1} \mathrm{H}-\mathrm{NMR}$ Spectrum of Compound $\mathbf{4 r}\left(\mathrm{CDCl}_{3}\right)$

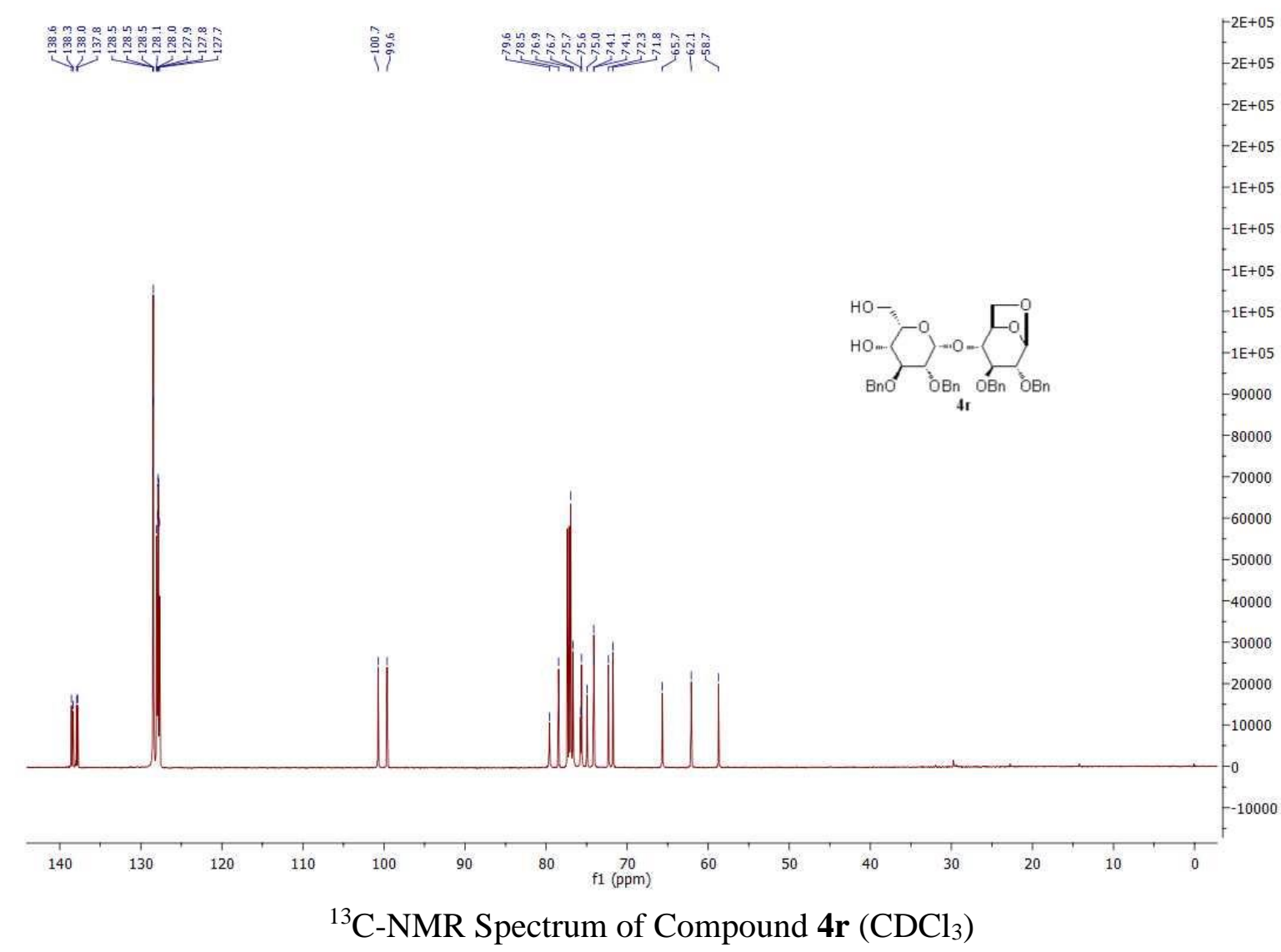




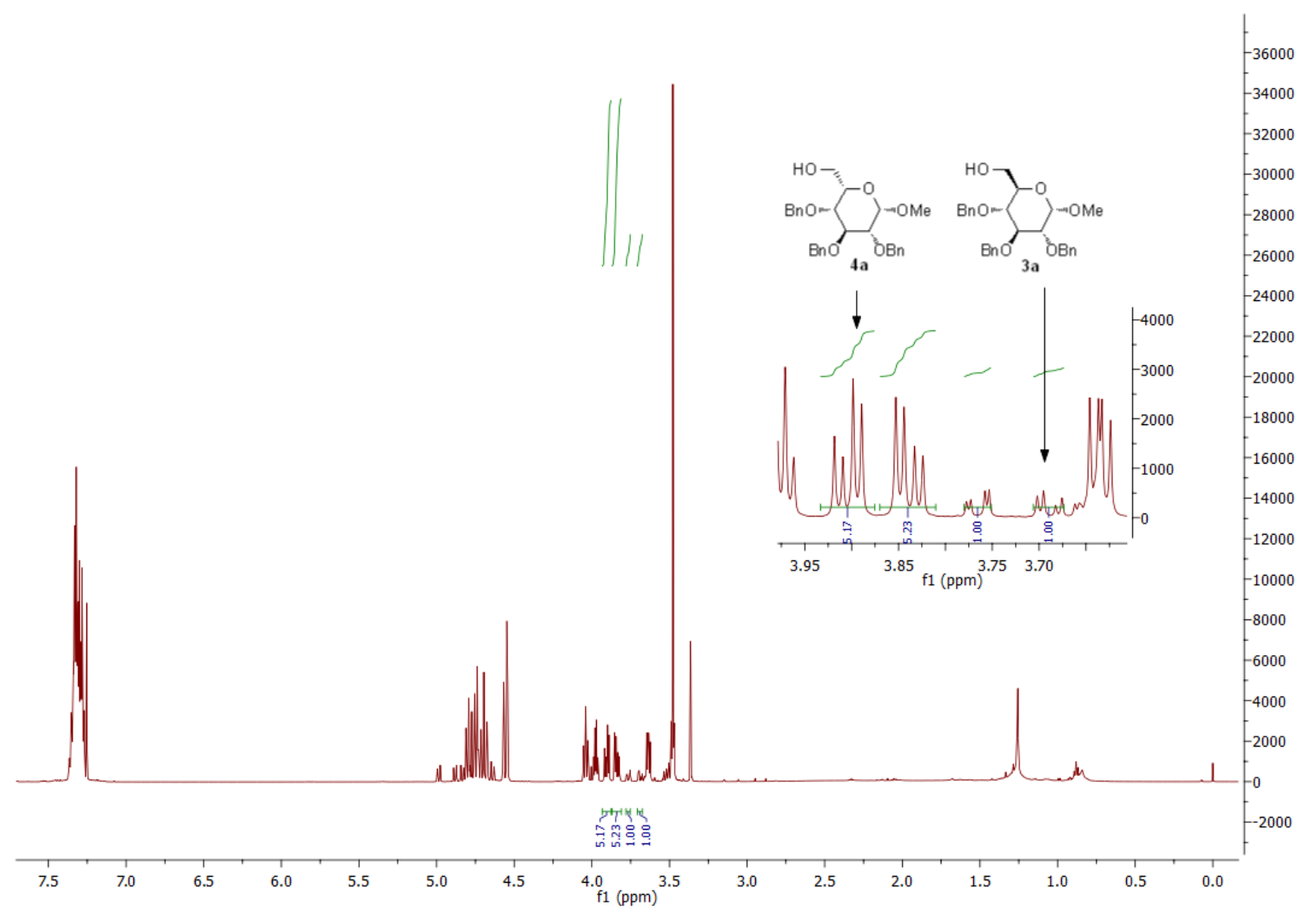

${ }^{1} \mathrm{H}-\mathrm{NMR}$ Spectrum of $\mathbf{3 a}$ and $\mathbf{4 a}$ mixture $(\mathbf{3 a}: \mathbf{4 a}=1: 5.2)\left(\mathrm{CDCl}_{3}\right)$

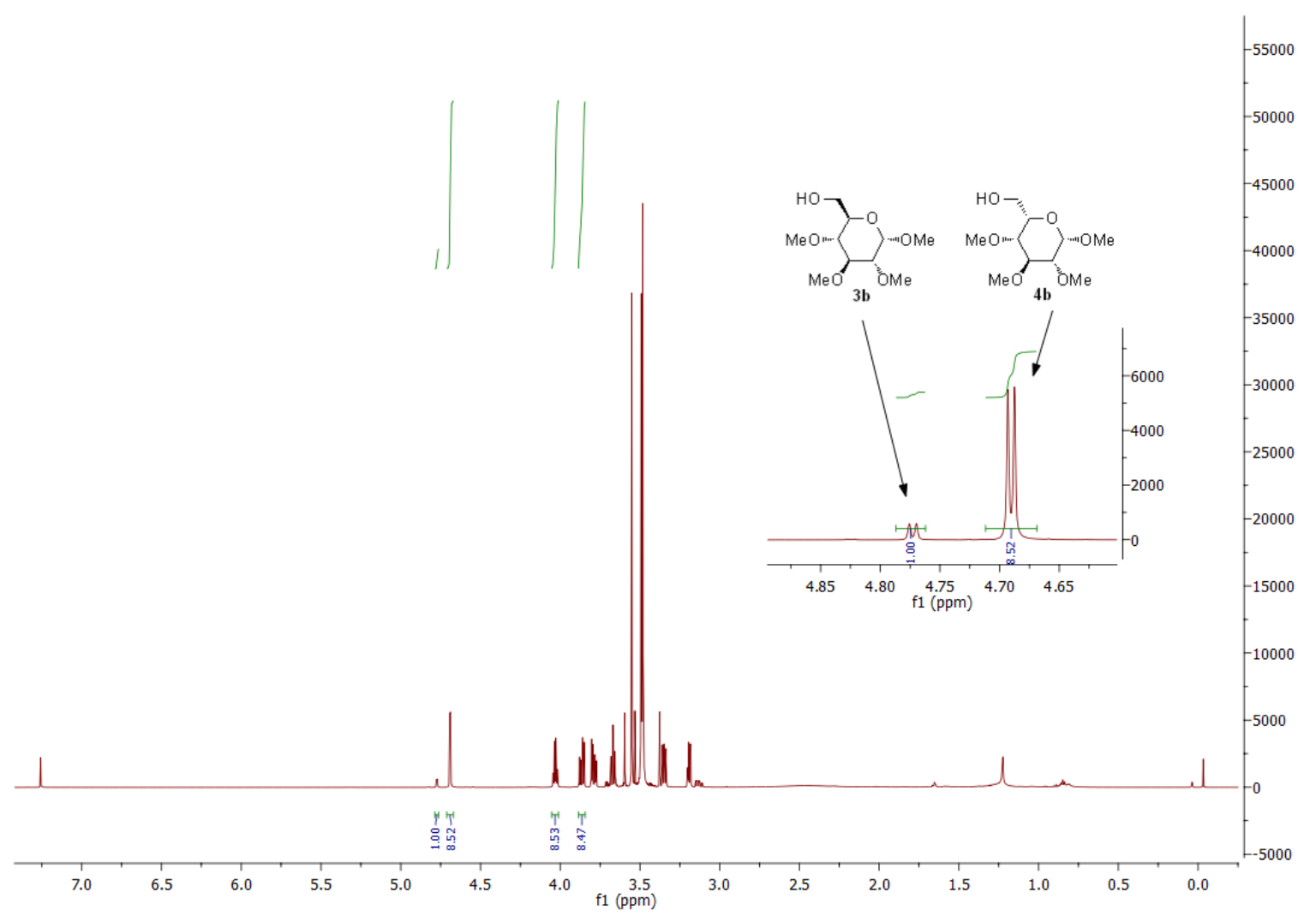

${ }^{1} \mathrm{H}-\mathrm{NMR}$ Spectrum of $\mathbf{3 b}$ and $\mathbf{4 b}$ mixture $(\mathbf{3 b}: \mathbf{4 b}=1: 8.5)\left(\mathrm{CDCl}_{3}\right)$ 


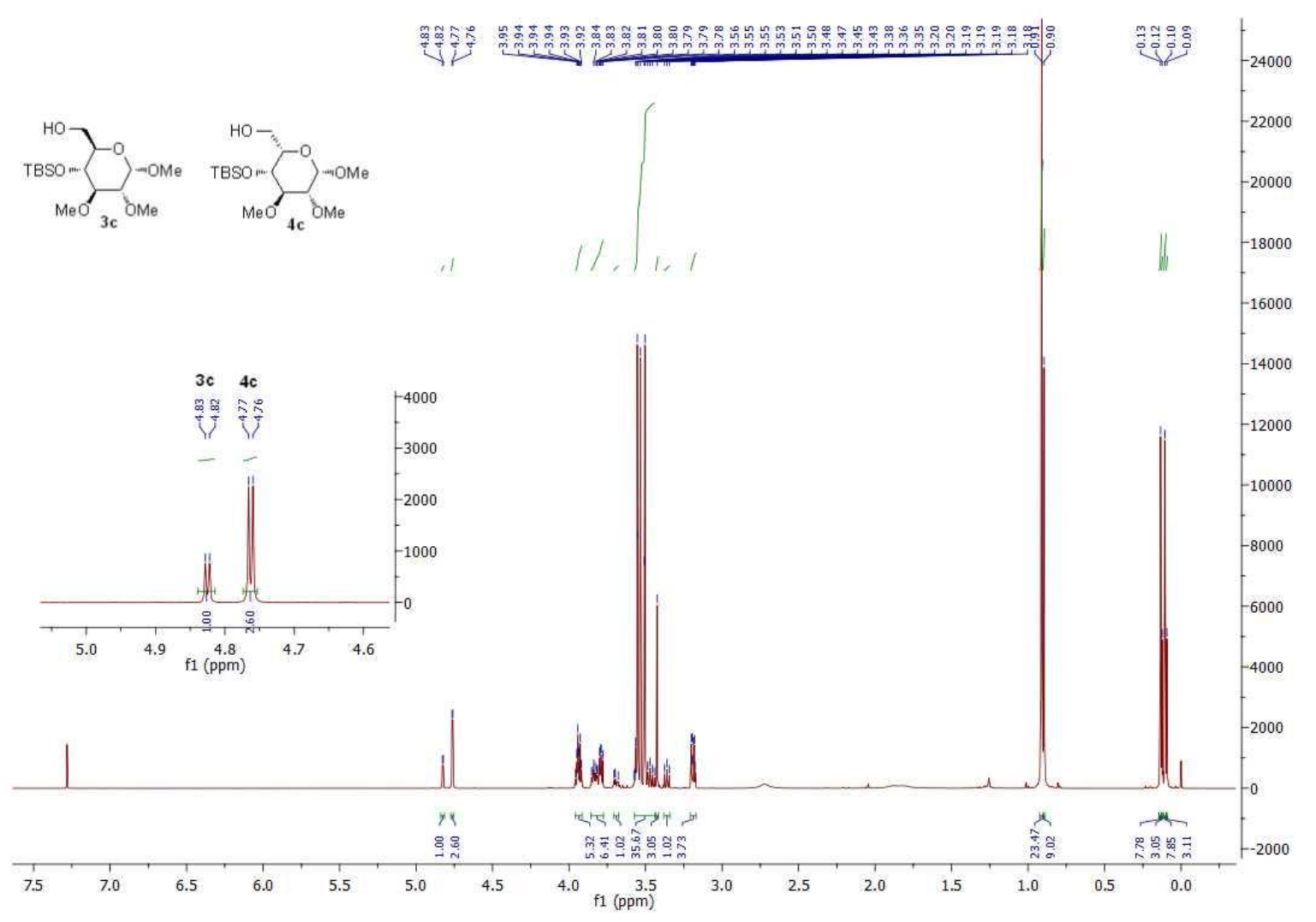

${ }^{1} \mathrm{H}-\mathrm{NMR}$ Spectrum of $\mathbf{3 c}$ and $\mathbf{4 c}$ mixture $(\mathbf{3 c}: \mathbf{4 c}=1: 2.6)\left(\mathrm{CDCl}_{3}\right)$

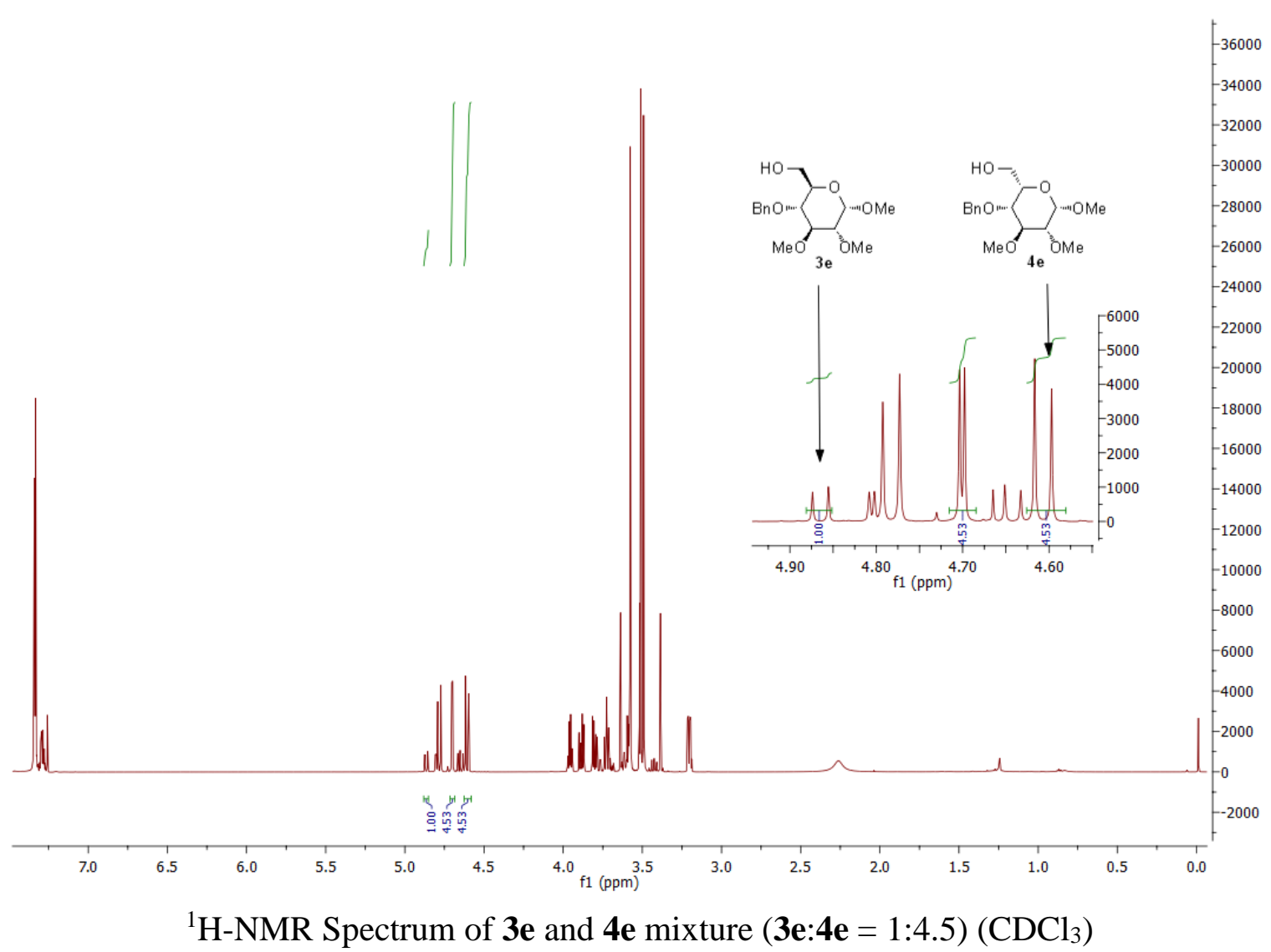




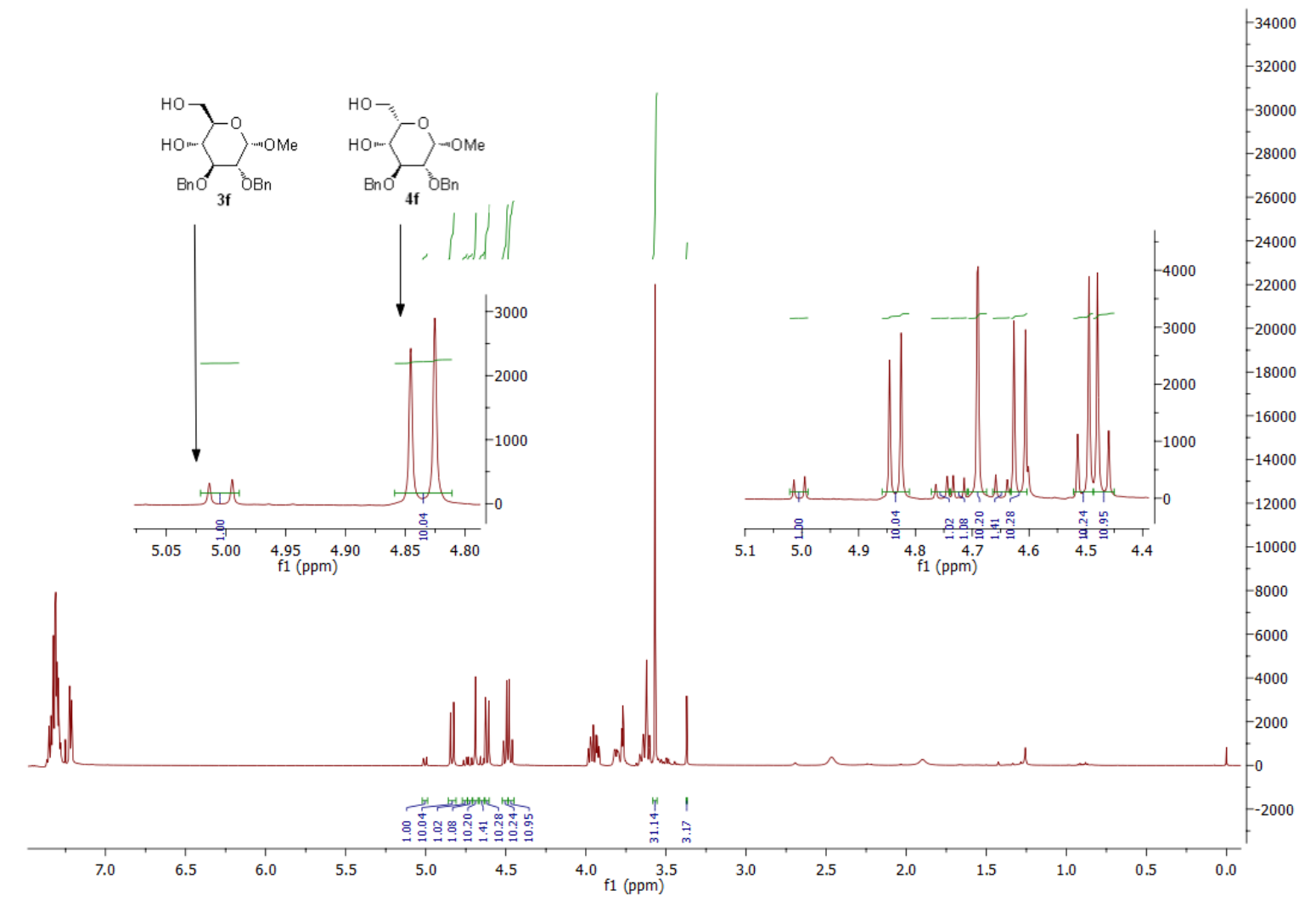

${ }^{1} \mathrm{H}-\mathrm{NMR}$ Spectrum of $\mathbf{3 f}$ and $\mathbf{4 f}$ mixture $(\mathbf{3 f}: \mathbf{4 f}=1: 10)\left(\mathrm{CDCl}_{3}\right)$

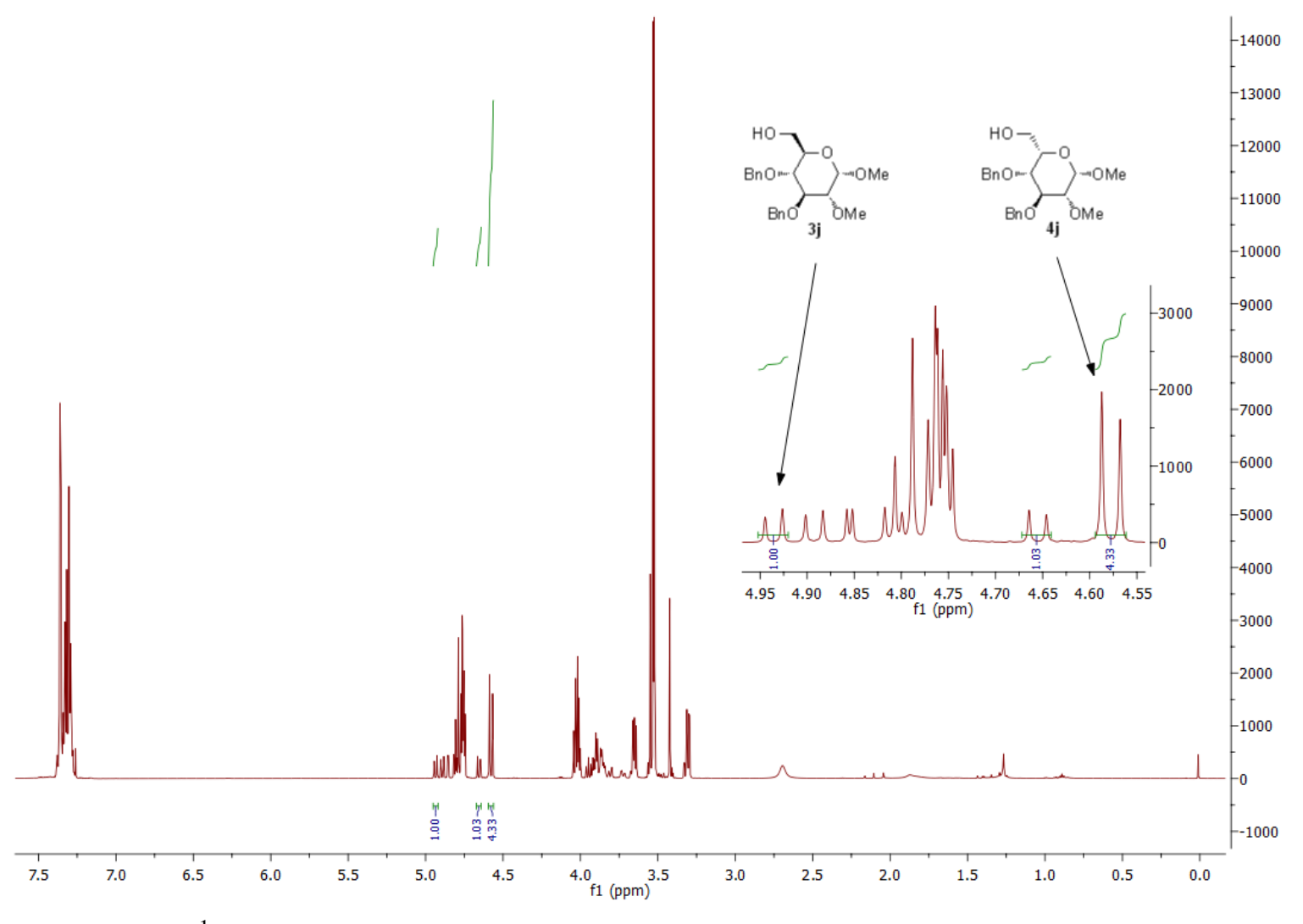

${ }^{1} \mathrm{H}-\mathrm{NMR}$ Spectrum of $\mathbf{3 j}$ and $\mathbf{4 j}$ mixture $(\mathbf{3 j}: \mathbf{4 j}=1: 4.3)\left(\mathrm{CDCl}_{3}\right)$ 


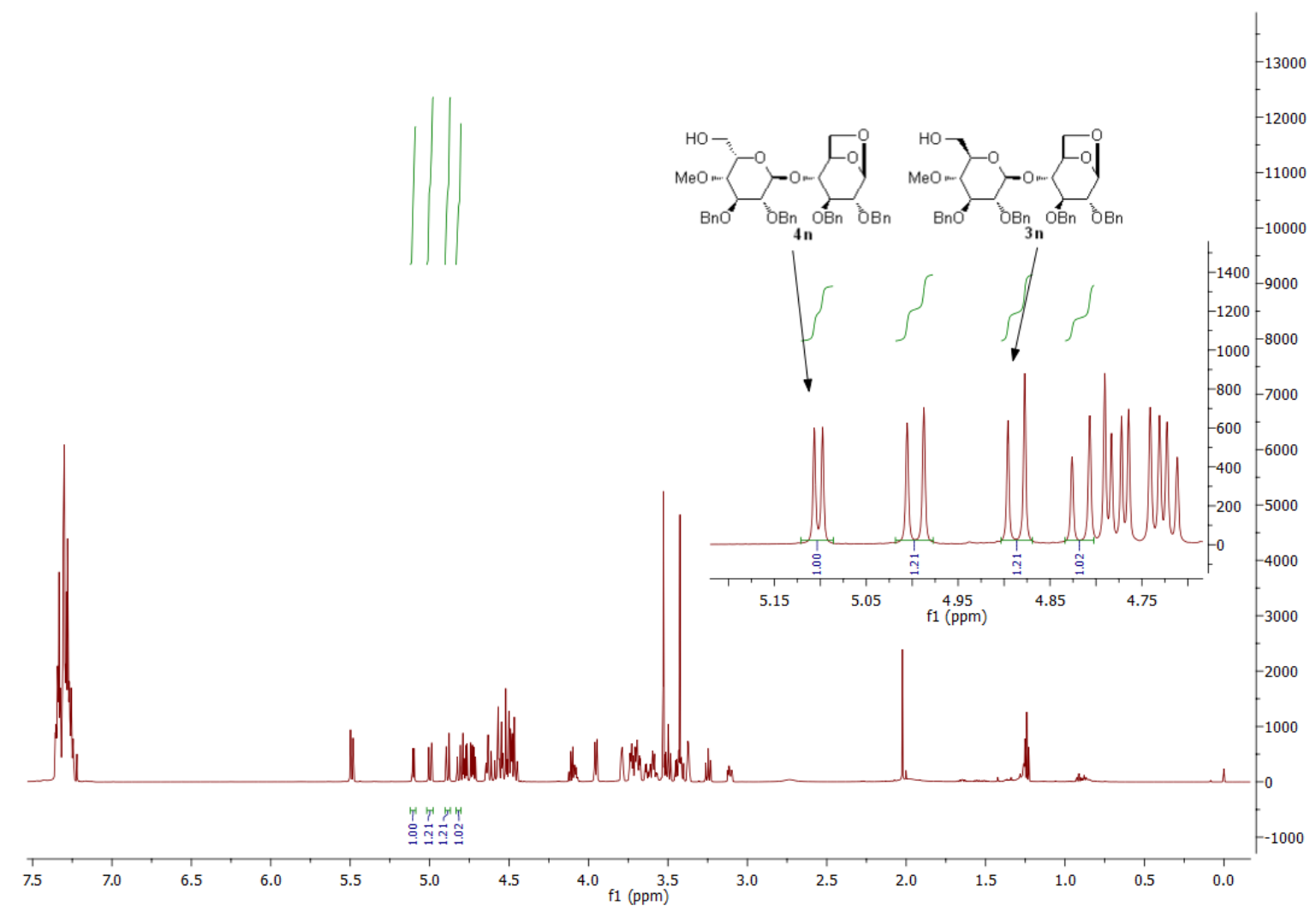

${ }^{1} \mathrm{H}-\mathrm{NMR}$ Spectrum of $\mathbf{3 n}$ and $\mathbf{4 n}$ mixture $(\mathbf{3 n}: \mathbf{4 n}=1.2: 1)\left(\mathrm{CDCl}_{3}\right)$ 